\title{
Solar Variability over the Last 9000 Years
}

\author{
Dissertation \\ zur Erlangung des mathematisch-naturwissenschaftlichen Doktorgrades \\ "Doctor rerum naturalium" \\ der Georg-August-Universität Göttingen \\ im Promotionsprogramm PROPHYS \\ der Georg-August University School of Science (GAUSS)
}

vorgelegt von

\section{Chi-Ju Wu}

aus Taipei, Taiwan

Göttingen, 2018 
Betreuungsausschuss

Prof. Dr. Ansgar Reiners

Institut für Astrophysik, Georg-August-Universität Göttingen, Germany

Dr. Natalie A. Krivova

Max-Planck-Institut für Sonnensystemforschung, Göttingen, Germany

Prof. Dr. Sami K. Solanki

Max-Planck-Institut für Sonnensystemforschung, Göttingen, Germany

School of Space Research, Kyung Hee University, Yongin, South Korea

Mitglieder der Prüfungskommision

Referent: Dr. Natalie A. Krivova

Max-Planck-Institut für Sonnensystemforschung, Göttingen, Germany

Korreferent: Prof. Dr. Ansgar Reiners

Institut für Astrophysik, Georg-August-Universität Göttingen, Germany

Weitere Mitglieder der Prüfungskommission:

Prof. Dr. Sami K. Solanki

Max-Planck-Institut für Sonnensystemforschung, Göttingen, Germany

Prof. Dr. Wolfram Kollatschny

Institut für Astrophysik, Georg-August-Universität Göttingen, Germany

Prof. Dr. Maarit J. Käpylä

Max-Planck-Institut für Sonnensystemforschung, Göttingen, Germany

Prof. Dr. Stefan Dreizler

Institut für Astrophysik, Georg-August-Universität Göttingen, Germany

Tag der mündlichen Prüfung: 23. 05. 2018 


\section{Bibliografische Information der Deutschen Nationalbibliothek}

Die Deutsche Nationalbibliothek verzeichnet diese Publikation in der Deutschen Nationalbibliografie; detaillierte bibliografische Daten sind im Internet über http: //dnb . d-nb . de abrufbar.

ISBN 978-3-944072-63-0

uni-edition GmbH 2018

http://www.uni-edition.de

(C) $\mathrm{Chi}-\mathrm{Ju} \mathrm{Wu}$

(c) This work is distributed under a
Creative Commons Attribution 3.0 License

Printed in Germany 



\section{Contents}

$\begin{array}{lr}\text { Summary } & 9\end{array}$

$\begin{array}{ll}\text { Zusammenfassung } & 11\end{array}$

1 Introduction $\quad 13$

1.1 Sun-Earth connection . . . . . . . . . . . . . . . . 13

1.2 Our Sun . . . . . . . . . . . . . . . . . . . 17

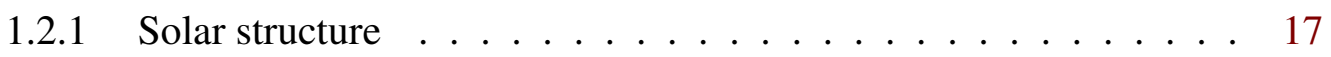

1.2.2 Solar magnetic activity . . . . . . . . . . . . . . . . . 20

1.2.3 Solar cycles . . . . . . . . . . . . . . . . 26

1.3 Solar total and spectral irradiance . . . . . . . . . . . . . . 27

1.3.1 Measurements . . . . . . . . . . . . . . 27

1.3.1.1 Measurements of TSI . . . . . . . . . . . 27

1.3.1.2 Measurements of SSI . . . . . . . . . . . . . . 29

1.3.2 Physical mechanisms of solar irradiance . . . . . . . . . . . . . . 31

1.3.3 Models of irradiance variability . . . . . . . . . . . . 32

1.3.3.1 Proxies . . . . . . . . . . . . 32

1.3.3.2 Proxy models ..................... 33

1.3.3.3 Semi-empirical models . . . . . . . . . . 34

1.4 SATIRE model . . . . . . . . . . . . . . . . . 35

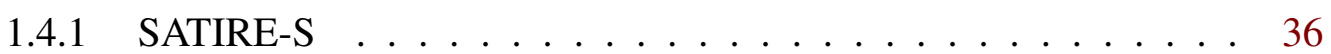

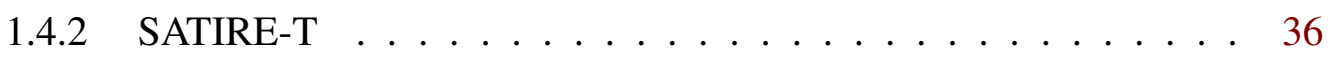

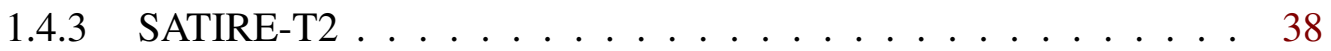

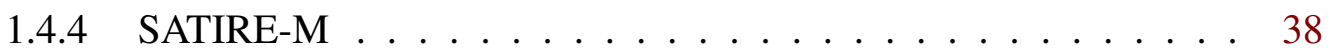

1.5 Thesis outline . . . . . . . . . . . . . . . . . . 39

2 Background of solar activity proxies $\quad 41$

2.1 Sunspot observations . . . . . . . . . . . . . . . . . 41

2.2 Geomagnetic field . . . . . . . . . . . . . . . . . . 45

2.3 Cosmic rays . . . . . . . . . . . . . . . . . . . 49

2.3.1 Heliospheric modulation . . . . . . . . . . . . . 50

2.3.2 Geomagnetic modulation (shielding effect) . . . . . . . . . 52

2.4 Cosmogenic isotopes . . . . . . . . . . . . . . . . . . . 54

2.4.1 Radiocarbon ${ }^{14} \mathrm{C} \ldots \ldots \ldots \ldots 56$

2.4.2 Radionuclide ${ }^{10} \mathrm{Be} \ldots \ldots \ldots . \ldots . \ldots 58$ 
3 Solar activity over nine millennia: A consistent multi-proxy reconstruction $\mathbf{6 1}$

3.1 Introduction . . . . . . . . . . . . . . . . . . 62

3.2 Data . . . . . . . . . . . . . . . . 64

3.2.1 Cosmogenic isotope records . . . . . . . . . . . . . 64

3.2.2 Geomagnetic data . . . . . . . . . . . . . . . . 64

3.3 Data processing . . . . . . . . . . . . . 66

3.3.1 Temporal synchronization of the records: Wiggle matching . . . . 66

3.3.1.1 Choice of wiggles . . . . . . . . . . . . . 67

3.3.1.2 Synchronization of the wiggles . . . . . . . . . 68

3.4 Reconstruction of the solar modulation potential . . . . . . . . . . 70

3.4.1 Reducing the series to the reference geomagnetic conditions . . . 72

3.4.2 Reconstruction based solely on ${ }^{14} \mathrm{C} \ldots \ldots 73$

3.4.3 Comparison between the long ${ }^{10} \mathrm{Be}$ and ${ }^{14} \mathrm{C}$ series . . . . . . . . 75

3.4.4 Reconstructions from individual series . . . . . . . . . . . 77

3.4.5 A combined-record reconstruction of $\phi \ldots \ldots 77$

3.4 .6 Full reconstruction . . . . . . . . . . . . . . . . . . 79

3.5 Reconstruction of sunspot number . . . . . . . . . . . . . 81

3.6 Conclusions . . . . . . . . . . . . . . . . . 85

4 Solar total and spectral irradiance reconstruction over the last 9000 years $\quad 89$

4.1 Introduction . . . . . . . . . . . . . . . . . . 90

4.2 Model description . . . . . . . . . . . . . . . . . . . . . . 92

4.2.1 The general concept of the SATIRE model . . . . . . . . . . . 92

4.2 .2 SATIRE-T . . . . . . . . . . . . . . . 93

4.2.2.1 Surface magnetic field . . . . . . . . . . 93

4.2.2.2 Filling factors . . . . . . . . . . . . 95

4.2.2.3 Model optimisation . . . . . . . . . . . 96

4.2 .3 SATIRE-M . . . . . . . . . . . . . . 97

4.2.3.1 Cosmogenic data . . . . . . . . . . . . . 97

4.2.3.2 Reconstruction of solar irradiance . . . . . . . . . . 99

4.3 Results . . . . . . . . . . . . . . . . . . . 102

4.3.1 Comparison with observations . . . . . . . . . . . . . 102

4.3.2 SATIRE-T reconstruction back to the Maunder minimum . . . . . 104

4.3.3 SATIRE-M reconstruction on millennial time scales . . . . . . 107

4.4 Summary . . . . . . . . . . . . . . . . . . . . 111

5 Postdicting Solar Cycles on Millennial Time Scale 113

5.1 Introduction . . . . . . . . . . . . . . . . . . . . . . 114

5.2 Cosmogenic isotope records . . . . . . . . . . . . . 115

5.3 Methodology . . . . . . . . . . . . . . 116

5.3 .1 Statistical approach . . . . . . . . . . . . . . . 117

5.3.2 Shape of solar cycle . . . . . . . . . . . . . . 120

5.3 .3 Phase adjustment . . . . . . . . . . . . . . . 121

5.3.4 Test simulation of the procedure . . . . . . . . . . . . 121

5.3.5 Sensitivity of the result to the decadal segmentation . . . . . . 123

5.4 Results . . . . . . . . . . . . . . . . . . . . . . 127 
5.4.1 Simulation of solar cycles over the last 9 millennia . . . . . . . 127

5.4.2 Reconstruction of solar irradiance on millennial time scale with solar cycles . . . . . . . . . . . . . . . . . . . 129

5.5 Summary . . . . . . . . . . . . . . . . . . . 134

6 Summary and outlook 137

6.1 Summary . . . . . . . . . . . . . . . . . . . 137

6.2 Outlook ............................. 140

$\begin{array}{ll}\text { Bibliography } & 143\end{array}$

A Appendix 173

A.1 Derivation of the magnetic flux in the SATIRE-M model . . . . . . . . 173

A.2 Derivation of the solar irradiance in the SATIRE-M model . . . . . . 176

$\begin{array}{ll}\text { Publications } & 179\end{array}$

$\begin{array}{ll}\text { Acknowledgements } & 181\end{array}$

$\begin{array}{ll}\text { Curriculum vitae } & 183\end{array}$ 



\section{Summary}

The solar activity has been observed to vary at various time scales, of which the 11-year solar cycle is the most prominent one. Since the Sun provides the main external energy to the Earth, knowledge of solar variability is highly crucial for understanding the influence of the Sun on the Earth's climate system.

One of the important measurements related to solar variability is the solar irradiance: the total solar irradiance (TSI) and the spectral solar irradiance (SSI). However, spacebased TSI/SSI measurements only cover the last four decades, which is unfortunately not sufficient for studying long-term solar variability and its influence on climate. Therefore, reconstructions of the solar irradiance on longer time scales are required.

Reconstructions of the solar irradiance require knowledge of proxies of solar magnetic activity. The only directly-observed solar quantity back to 1610 is the group sunspot number, while one has to rely on indirect proxies going further back in time. The commonly used indirect proxy is the concentration of the cosmogenic isotopes $\left({ }^{14} \mathrm{C}\right.$ and $\left.{ }^{10} \mathrm{Be}\right)$ retrieved from natural archives. Cosmogenic isotopes are produced in the upper terrestrial atmosphere by the impinging galactic cosmic rays, whose flux is modulated by the heliospheric magnetic field. The signals in the ${ }^{14} \mathrm{C}$ record are well globally-mixed while in the ${ }^{10} \mathrm{Be}$ records they are highly subjected to the local climate.

We constructed the first multi-isotope composite based on one global ${ }^{14} \mathrm{C}$ and six local ${ }^{10} \mathrm{Be}$ records, using a new Bayesian approach (Chap. 3). All six ${ }^{10} \mathrm{Be}$ records were first synchronized with respect to the ${ }^{14} \mathrm{C}$ record using a wiggle-matching method. Next a Monte Carlo simulation was performed to search for that solar modulation potential which best fits all the available isotope data sets at any given time. This composite is considered more robust compared to other composites constructed linearly. Hence, it is further used in this thesis as a proxy of solar magnetic activity on a millennial time scale.

In this thesis, we use two SATIRE (Spectral And Total Irradiance REconstruction) versions, SATIRE-T and SATIRE-M, to reconstruct the long-term changes in the solar irradiance. The SATIRE-T model uses the sunspot number to deduce the evolution of the solar surface magnetic components and to reconstruct the TSI/SSI back to the beginning of the 17th century. The SATIRE-M model employs the cosmogenic isotopes as proxies of solar activity to reconstruct the TSI/SSI over the Holocene.

Since the SATIRE-M model is based partially on the SATIRE-T model, we first revisited the SATIRE-T model with various modifications (Chap. 4). With these improvements, the free parameters in the SATIRE-T model are constrained and further employed in the SATIRE-M model. Next, we use the SATIRE-M model and the first multiisotope composite to reconstruct the solar irradiance over the last 9000 years. This is the first SSI reconstruction that not only uses physics-based models to describe all involved non-linear physical processes, but also bases on a multi-isotope composite. The TSI/SSI 
reconstructions have been recommended for studies of long-term climate changes within the Palaeoclimate Modelling Intercomparison Project-phase 4 (PMIP4).

Due to the sampling and quality of the cosmogenic isotope data, the reconstructions with the SATIRE-M model have only a resolution of 10 years, which, unfortunately, might cause biases in the climate models. Therefore, we developed a statistical approach to simulate the quasi 11-year solar cycle from the decadally-averaged sunspot numbers (Chap. 5). This is done by characterising the solar cycle properties and finding the linear relationships between these properties and the decadally-averaged sunspot numbers. This simulated sunspot number series with 11-year solar cycles has annual resolution, and is further employed in the SATIRE model to reconstruct the annual values of TSI/SSI over the Holocene.

The TSI/SSI reconstructions with simulated cycles are consistent with the reconstructions based on the directly-observed sunspot numbers. This final solar irradiance reconstruction has been provided as a solar forcing input to climate models. This hopefully will help us to better understand the degree of the solar influence on the Earth's climate on long time scales. 


\section{Zusammenfassung}

Die Aktivität der Sonne variiert auf verschiedenen Zeitskalen. Der 11-jährige Sonnenzyklus ist der herausragendste Ausdruck dieder Variabilität. Da die Sonne die wichtigste externe Energiequelle für die Erde ist, ist die Kenntnis der Schwankungen ihrer Aktivität von größter Wichtigkeit für das Verständnis des Einflusses der Sonne auf das Erdklima.

Zwei der wichtigsten Größen, die die Sonnenvariabilität widerspiegeln, sind die gesamte Bestrahlungsstärke (total solar irradiance, TSI) und die spektral aufgelöste Bestrahlungsstärke (spectral solar irradiance, SSI). Weltraumbasierte Messungen der Bestrahlungsstärke decken nur die letzten vier Jahrzehnte ab, was für Studien der langfristigen Sonnenvariabilität und deren Einfluss auf das Klima unzureichend ist. Daher ist es notwendig, die Bestrahlungsstärke der Sonne über längere Zeiträume zu rekonstruieren.

Proxies für die magnetische Aktivität der Sonne ermöglichen die Rekonstruktion der Bestrahlungsstärke. Aufzeichnungen der Anzahl der Sonnenfleckengruppen reichen bis zurück ins Jahr 1610 und sind die einzige direkt beobachtbare Kenngröße. Zu noch früheren Zeiten, kann auf indirekte Anzeiger wie die Konzentration der kosmogenen Isotope ${ }^{14} \mathrm{C}$ und ${ }^{10} \mathrm{Be}$, die aus natürlichen Archiven gewonnen wird, zurückgegriffen werden. Kosmogene Isotope werden in der oberen Erdatmosphäre durch auftreffende galaktische kosmische Strahlung, deren Strahlungsfluss vom heliosphärischen Magnetfeld beeinflusst wird, produziert. Die Signale in ${ }^{14} \mathrm{C}-$ Messreihen sind global wohldurchmischt, wohingegen ${ }^{10} \mathrm{Be}-M e s s r e i h e n$ stark vom lokalen Klima abhängen.

In Kap. 3 wird die erste Zusammenstellung aus mehreren Isotopen mit einer neuen, auf Bayesscher Statistik beruhenden Methode konstruiert. Diese Zusammenstellung besteht aus einer globalen ${ }^{14} \mathrm{C}$-Messreihe und sechs lokalen ${ }^{10} \mathrm{Be}-\mathrm{Messreihen}$. Alle ${ }^{10} \mathrm{Be}-$ Messreihen werden zuerst mithilfe einer Wiggle-Matching-Methode an die ${ }^{14} \mathrm{C}$-Messreihe angepasst. Danach wird mittels einer Monte-Carlo-Simulation das Modulationspotenzial der Sonne bestimmt, welches zu dem jeweiligen Zeitpunkt am besten zu den Isotopendaten passt. Daher wird die neue Rekonstruktion im weiteren Verlauf der Arbeit als Näherungsvariable für die magnetische Aktivität der Sonne verwendet.

In dieser Arbeit werden zwei SATIRE-Varianten (Spectral And Total Irradiance REconstruction; Rekonstruktion der gesamten und spektral aufgelösten Bestrahlungsstärke) verwendet, um die langfristigen Änderungen der Bestrahlungsstärke zu rekonstruieren, nämlich SATIRE-T und SATIRE-M. SATIRE-T verwendet die Anzahl der Sonnenflecken, um die Entwicklung der magnetischen Komponenten der Sonnenoberfläche herzuleiten und die Bestrahlungsstärke bis zurück ins 17. Jahrhundert zu rekonstruieren. SATIRE-M verwendet die kosmogenen Isotope ${ }^{14} \mathrm{C}$ und ${ }^{10} \mathrm{Be}$ als indirekte Anzeiger für die Sonnenaktivität, um die Bestrahlungsstärke im Holozän zu rekonstruieren.

Da SATIRE-M teilweise auf SATIRE-T basiert, wird SATIRE-T zunächst an einigen Stellen verbessert (Kap. 4). Nach diesen Verbesserungen werden die freien Parameter 
in SATIRE-T bestimmt und in SATIRE-M weiterverwendet. Wir verwenden SATIRE$\mathrm{M}$ und die Kombination mehrerer Isotope aus dieser Arbeit, um die Bestrahlungsstärke über die letzten 9000 Jahre zu rekonstruieren. Dies ist die erste Rekonstruktion der spektral aufgelösten Bestrahlungsstärke, die nicht nur physikalische Modelle zur Beschreibung aller beteiligten nichtlinearen physikalischen Prozesse verwendet, sondern auch auf der Kombination mehrerer Isotope basiert. Die rekonstruierten Bestrahlungsstärken wurden im Rahmen des Palaeoclimate Modelling Intercomparison Project (PaläoklimaModellierungsvergleichs-Projekt 4; PMIP4) für Untersuchungen langfristiger Klimaveränderungen empfohlen.

Da die kosmogenen Isotopendaten eine Auflösung von 10 Jahren haben, hat auch die Rekonstruktion der Irradianz mit SATIRE-M die gleiche Auflösung, die Verzerrungen in Klimamodellen verursachen könnte. Daher wurde ein statistischer Ansatz entwickelt, um den ungefähr 11-jährigen Sonnenzyklus aus den über ein Jahrzehnt gemittelten Sonnenfleckenzahlen zu extrahieren (Kap. 5). Dazu werden die Eigenschaften des Sonnenzyklus charakterisiert und lineare Beziehungen zwischen diesen und den über ein Jahrzehnt gemittelten Sonnenfleckenzahlen aufgestellt. Diese simulierte Sonnenflecken-Zahlenreihe mit 11-jährigen Sonnenzyklen hat eine Auflösung von einem Jahr. Sie wird im SATIREModell benutzt, um die jährlichen Werte der Bestrahlungsstärke über das Holozän zu rekonstruieren.

Die Rekonstruktion der Bestrahlungsstärke mit simulierten Sonnenzyklen sind mit den Rekonstruktionen vereinbar, die auf den direkt beobachteten Sonnenfleckenzahlen basieren. Diese abschließende Rekonstruktion der Bestrahlungsstärke wurde als Strahlungsantrieb für Klimamodelle bereitgestellt. Dies wird hoffentlich helfen, den Grad des Einflusses der Sonne auf das Erdklima über lange Zeiträume besser zu verstehen. 


\section{Introduction}

With the goal of better understanding the past variability of the Earth's climate changes, this thesis is dealing with solar variability on long time scales (i.e., centuries to millennia), which is one of the drivers of the Earth's climate, at least in the past. Therefore, at the beginning of this thesis, Sect. 1.1, we will briefly address the relationship between our Sun and the Earth's climate system. In section 1.2, the basic physics and the background knowledge of the Sun will be given. Next, section 1.3 reviews our knowledge of the solar radiative output (solar irradiance), its space-borne measurements and the models.

In this thesis we will later use the SATIRE (Spectral And Total Irradiance REconstruction) model to reconstruct the solar irradiance. Hence, Sect. 1.4 gives a more detailed overview of this model. The proxies of solar activity we use in the two SATIRE models and their relevant background knowledge will be described in the next chapter.

\subsection{Sun-Earth connection}

The Sun has a strong influence on the Earth in many respects (from magnetosphere to the climate) and on various time scales (from days to millennia). The Earth's magnetosphere is modulated by the solar wind (Sect. 2.2) in such a way that the near-Earth space (magnetosphere, ionosphere, thermosphere) is sensitive to the changes in the heliosphere and events originating on the Sun. The events produced by short-term variations of the Sun and their effects on the near-Earth environment are usually dealt by space weather. For instance, shock waves created by coronal mass ejections (CMEs) are observed to cause geomagnetic storms. The radio bursts produced by solar flares could disrupt the radio communication system on the Earth. The solar energetic particles (SEPs) could also induce strong aurorae in the higher latitudes, sometimes even pose threats to satellites, electricity facilities, resulting in power shortages (e.g., the Carrington event in 1859).

Solar variations have also great influence on the Earth on longer time scales, particularly on the Earth's climate, because the Sun is the main source of energy to the Earth. By analysing the sunspot numbers records (Sect. 2.1), the terrestrial cosmogenic isotopes data (Sect. 2.4) and the climate-related quantities (such as global sea surface temperature (SST) and tree growth widths), the relationships between the Sun and the Earth's climate have been observed (e.g., Schneider and Mass 1975; Eddy 1976; Reid 1987; Bond et al. 2001). Friis-Christensen and Lassen (1991) has also found the link between the northern hemisphere temperature and the solar cycle length.

Recently, various surface climate records (e.g., corals, stalagmites, marine sediments) in the past have suggested that the Earth's climate might be affected by the long-term variations of solar activity. One of the classical examples of the relationship between the 


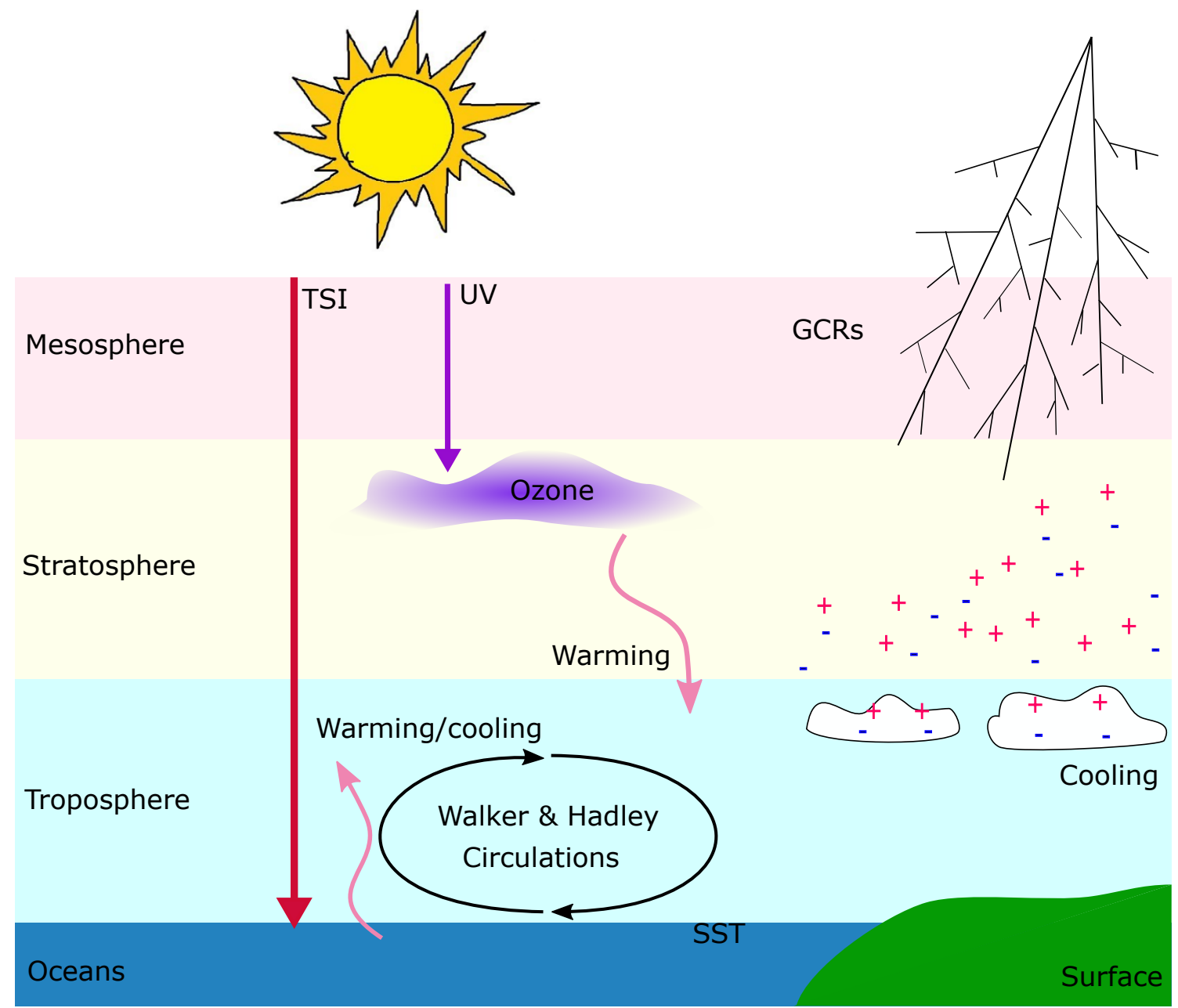

Figure 1.1: Schematic indicating the potential mechanisms that might influence the Earth's climate (details see text). Reproduced after Gray et al. (2010).

solar activity and the Earth climate is the Maunder minimum, a period between 1645 1715 AD. Sunspots seemed to have disappeared during the Maunder minimum, which was coincident with a long cold period in North America and Western Europe between mid-15th to mid-19th century (e.g., Mann 2002), also known as the Little Ice Age (LIA). LIA also covers another extreme of low solar activity between 1640 - 1550 AD (Spörer minimum). Nevertheless, Jungclaus et al. (2010) has suggested that the LIA might have been caused by volcanism activities. The particles and aerosols injected along with the volcano eruptions into the stratosphere could cover the Earth's surface and block a great percentage of the incoming solar irradiance, causing lower surface temperature.

There are also other potential astronomical mechanisms of influencing the Earth's climate. For instance, the Earth's orbit parameters change with time. The precession, the obliquity, and the eccentricity parameters vary with periodicities of $\approx 23000, \approx 41000$ and $\approx 100000$ years, respectively (Paillard 2001; Crucifix et al. 2006). With the change of these orbit parameters, the Sun-Earth distance and the incident angle of the sunlight also vary. The collective effect of these changing parameters on the Earth climate is known as the Milankovitch cycle, which is on time scales of a few tens of thousands of years and is 
the prime cause of the occurrence of the glacial/interglacial periods. Additionally, cosmic rays have also been proposed to affect the Earth global climate (e.g., Ney 1959; Dickinson 1975; Svensmark and Friis-Christensen 1997; Marsh and Svensmark 2000a,b; Dorman 2012). The ions produced by the cosmic rays have been suggested to act as condensation nuclei and further cluster and become cloud condensation nuclei (CCN). The CCN could enhance the formation of the clouds (so-called cloud nucleation), increase the cloud cover area, and further cool the surface temperature. Although the correlation between the cloud cover and the cosmic ray intensity has been observed by Marsh and Svensmark (2000a), a solid physical connection between the cosmic rays and the cloud formation is lacking and the theory is still under debate. The Cosmics Leaving Outdoor Droplets (CLOUD) experiments (Kirkby et al. 2011) at CERN (European Organization for Nuclear Research) showed a very limited effect of cosmic ray strength on the CCN formation (Svensmark et al. 2016; Dunne et al. 2016; Gordon et al. 2017). Consequently, on the time scales we are concerned with in this thesis, i.e., from centennial to millennial, the solar radiative flux is still the prime solar source of influence on the global climate.

The two possible mechanisms by which the solar irradiance affects the Earth climate system: the top-down and the bottom-up effects, are described below and summarized in Fig. 1.1.

- Top-down mechanism: The chemical processes in the terrestrial atmosphere are highly wavelength and altitude-dependent. Particularly the stratosphere is sensitive to wavelengths shorter than $350 \mathrm{~nm}$ (viz., UV band). Oxygen molecules absorb the UV radiation in the Herzberg continuum $(200-242 \mathrm{~nm})$ and produce oxygen atoms and ozone. This oxygen photolysis process is responsible for the formation of ozone and heating up the stratopause region. Moreover, ozone is destroyed after absorbing the UV radiation in the Hartley-Huggins band $(200-315 \mathrm{~nm})$. This process is called photodissociation of ozone, which provides a strong radiative heating in the lower mesosphere and the upper stratosphere. Simulations show that the changes in the UV irradiance with the solar cycle affect the stratosphere patterns (Haigh 1996, 1999; Shindell et al. 1999; Larkin et al. 2000; Matthes et al. 2006; Haigh 2007). Perturbations in the stratosphere also influence the troposphere (Gillett and Thompson 2003; Scaife et al. 2005), where the main climate system performs. This stratospheric-induced heating propagates downwards to the troposphere and further affects the climate system. It is, therefore, called the "top-down UV effect" (Kodera and Kuroda 2002).

- Bottom-up mechanism: After the UV is absorbed by the Earth's ozone layer, the remaining solar irradiance (mainly visible and IR radiation) is mostly absorbed in the cloud-free subtropical regions. The oceans in these regions are heated and vaporized, which enhances the humidity in the air and strengthens the Hadley and the Walker circulations. The Hadley (cell) circulation is a global tropical circulation driven by the uprising air in the tropical region and the converging air in the subtropical region. A strong Hadley circulation results in a larger latitudinal extent and stronger trade winds (in the northern hemisphere, the warm air falls down to the surface at around latitude $30^{\circ}$ and moves south-eastward due to the Coriolis force). The Walker (cell) circulation is driven by the unevenly distributed heat in the eastern and western Pacific ocean. A strong Walker circulation results in a 
higher pressure in the eastern Pacific ocean and lower pressure in the west, leading to stronger monsoon seasons in the western Pacific countries and cooler SST in the eastern Pacific ocean caused by the enhancing upwelling current from the bottom of the ocean. This phenomenon is the so-called La Niña. To the contrary, a weaker Walker circulation results in warmer SST in the eastern Pacific ocean, causing flood in Peru and Ecuador and drought in south-east Asia and Australia (El Niño condition). This collective climate response to the absorption of TSI at or around the surface region is therefore called the "bottom-up TSI effect" (Cubasch et al. 1997; van Loon et al. 2007; Meehl et al. 2009).

It is important to note that the response of Earth's climate system to the solar radiative flux involves complex atmospheric circulations and feedback mechanisms. Therefore, both effects might only provide an initial trigger for an overall complexity in the climate system.

The concept of radiative forcing (RF) is widely used to estimate and analyse the response of the Earth surface temperature to the perturbations in the energy budget. RF is defined as the energy change of the perturbing factors, which influence the energy balance between the incoming solar irradiance and the emission by the Earth's atmosphere. These perturbing factors are called the RF agents, such as the greenhouse gases (GHGs, mainly methane and carbon dioxide), aerosols and clouds. The RF has units of $\left[\mathrm{W} / \mathrm{m}^{2}\right]$. A positive RF, such as an increasing amount of GHGs which absorb IR radiation and re-emit it back to the Earth's surface, results in a global temperature increase. A negative RF, such as an increase amount of aerosol particles which reflect the incoming solar irradiance, results in a global cooling effect. The change in the RF is found to have a linear relationship to the change in the surface temperature $\left(T_{\mathrm{s}}\right)$ :

$$
\Delta T_{\mathrm{s}}=\lambda_{\mathrm{s}} \Delta R F
$$

where $\lambda_{\mathrm{s}}$ is the climate sensitivity parameter with a typical value ranging from $0.3-1.0$ $\mathrm{K} /\left(\mathrm{W} / \mathrm{m}^{2}\right.$ ) (Haigh 2007) with a best estimate between $0.6-0.8 \mathrm{~K} /\left(\mathrm{W} / \mathrm{m}^{2}\right.$ ) (Solomon et al. 2007; Le Treut 2012). Note that since the Earth reflects about $30 \%$ of the incoming solar irradiance and re-distributes the absorbed irradiance over the global spherical surface, the change in the RF is not one to one correlated with the change in the TSI. For instance, a $1.0 \mathrm{~W} / \mathrm{m}^{2}$ increase in the TSI only results in a $0.175 \mathrm{~W} / \mathrm{m}^{2}$ increase in the RF, which implies a $0.1 \mathrm{~K}$ increase in the surface temperature (by taking $\lambda_{\mathrm{s}}=0.6$ into account).

It has now been generally agreed within the scientific community that the recent global warming is mostly caused by the release of GHGs, which are produced by the large amount of fossil fuel burning (Solomon et al. 2007). Nevertheless, to better estimate the level of anthropogenic factors on the Earth's climate, a good understanding of the natural cause (e.g., solar irradiation) needs to be taken into account. Since solar irradiance is an important energy forcing inputs in many climate models (Hansen 2000; Haigh 2001, 2003, 2007; Gray et al. 2010; Jungclaus et al. 2010; Schmidt et al. 2011; Jungclaus et al. 2016; Matthes et al. 2017), reliable reconstructions of both TSI and SSI further back into the past are needed. 


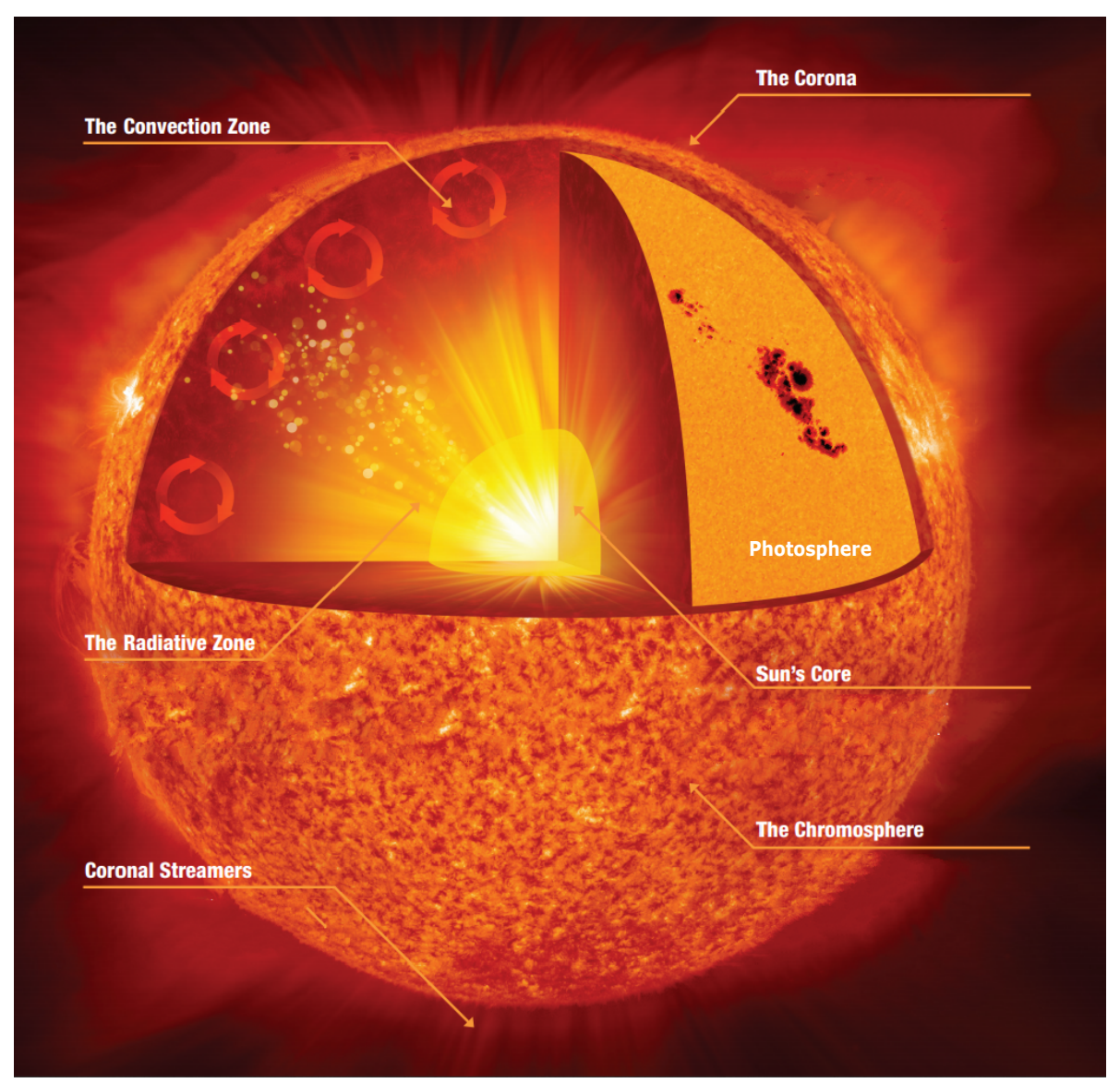

Figure 1.2: Schematic illustration of the solar structure: from the innermost part (core) through radiative zone, convection zone, photosphere, chromosphere, transition region, to the corona. Courtesy of NASA/Jenny Mottar.

\subsection{Our Sun}

The Sun is located at the centre of the solar system and contributes $99.86 \%$ of the solar system's total mass. It is a middle-aged $(\approx 4.6 \mathrm{Gyr})$ star sitting on the main sequence in the Hertzsprung-Russell diagram. It has a radius, $R_{\odot}$, of $695 \mathrm{Mm}$, and its mass, $M_{\odot}$, is about $2 \times 10^{30} \mathrm{~kg}$. The solar luminosity, $L_{\odot}$, which is defined as the total radiated energy from the entire solar surface, is about $3.8 \times 10^{26} \mathrm{~W}$.

\subsubsection{Solar structure}

The Sun is stratified in many layers with their unique physical features. The solar structure from the core to the corona is schematically illustrated in Fig. 1.2. The core has a radius of $0.2 R_{\odot}$. The density and the temperature of the core are so high $\left(150 \mathrm{~g} / \mathrm{cm}^{3}\right.$ and $1.57 \times 10^{7}$ $\mathrm{K}$, respectively) that the thermonuclear processes take place. Energy is generated mainly through proton-proton ( $\mathrm{p}-\mathrm{p}$ ) chain reaction (only less than $1 \%$ from the Carbon-NitrogenOxygen (CNO) cycle). This energy is carried outwards by photons travelling through the radiative zone, which is between $0.2-0.71 R_{\odot}$. In this region, the temperature decreases with distance from the core and reaches about $5 \times 10^{5} \mathrm{~K}$ at the top of the radiative zone. 


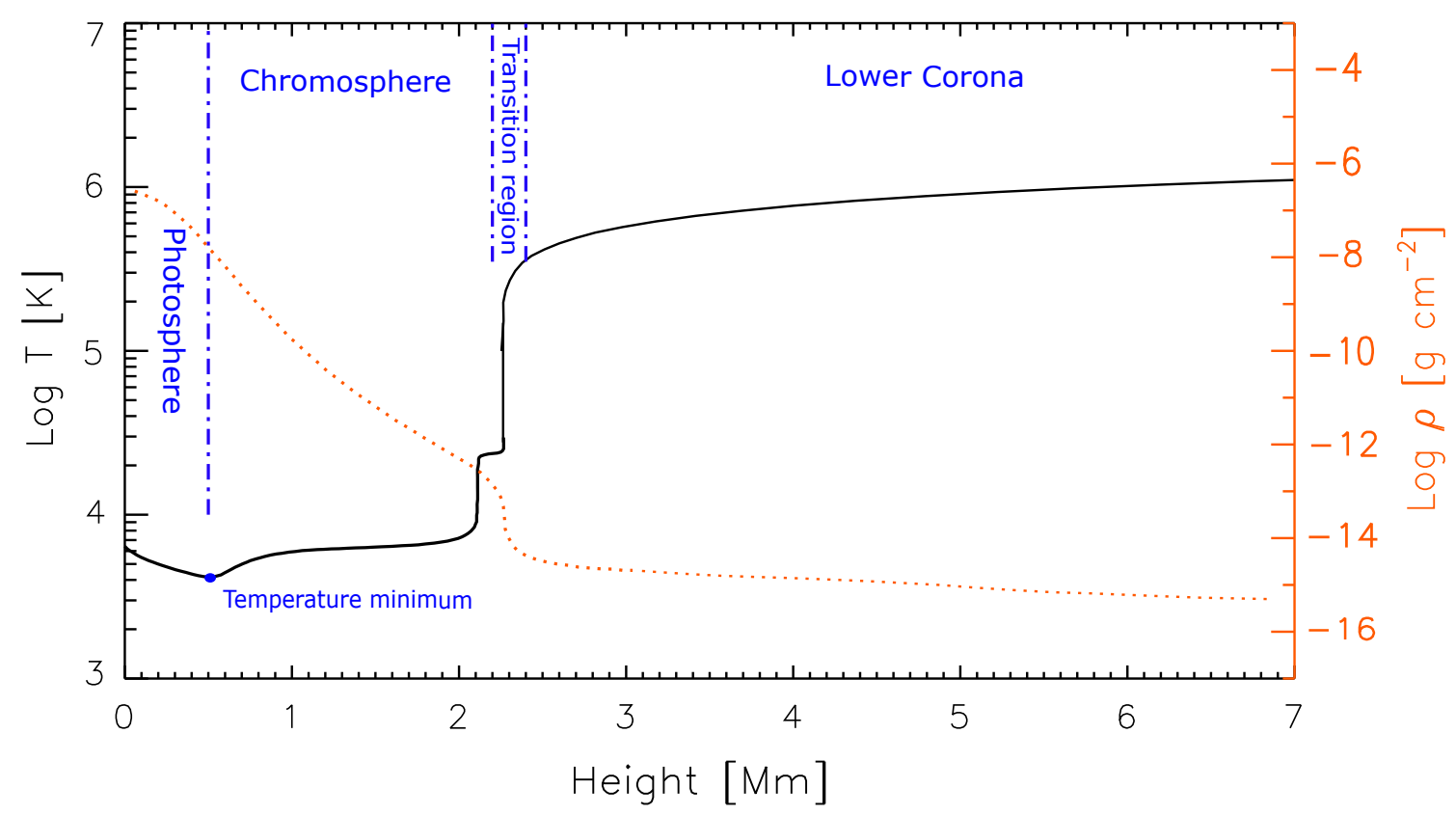

Figure 1.3: One dimensional profiles of the temperature (black, left y-axis) and density (red, right y-axis) in the solar atmosphere (above the surface, $\tau_{5000 \AA}=1$ ). The temperature and density are based on Reeves et al. (1977); Vernazza et al. (1981) and Avrett and Loeser (1992).

The region from $0.71 R_{\odot}$ outward is called the convection zone (Stix 2002). Energy in this region is transported by the turbulent convection, which can be observed as a granulation pattern at the photosphere (solar surface). Since the Sun has no solid "surface", the solar surface is usually defined as the layer with unity continuum optical depth at $500 \mathrm{~nm}$ (i.e., $\left.\tau_{5000 \AA}=1\right)$.

Above the solar surface is the solar atmosphere, which is usually divided into four layers (the photosphere, the chromosphere, the transition region and the corona) based on their physical properties. The 1-D density and temperature stratification profiles of the solar atmosphere are shown in Fig. 1.3. It is seen that the temperature falls to about $6 \times 10^{3} \mathrm{~K}$ in the photosphere and reaches the temperature minimum of $4000 \mathrm{~K}$ at $\approx 500 \mathrm{~km}$ above the solar surface. After reaching the temperature minimum, the temperature of the atmosphere increases first slowly with height in the lower to the middle chromosphere and then drastically in the upper chromosphere and in the transition region. Eventually, the temperature rises abruptly to $1-3 \times 10^{6} \mathrm{~K}$ in the corona. The coronal heating problem is still one of the greatest unsolved mysteries in solar physics (Parnell and De Moortel 2012). There have been different proposed mechanisms and supportive observations to explain this drastic temperature increase in the upper solar atmosphere. These mechanisms include acoustic waves proposed by Schwarzschild (1948) and Biermann (1948), small-scale magnetic field reconnection (nanoflares, Parker 1972, 1983, 1988; Hudson 1991; Priest et al. 2002), and magnetohydrodynamics waves (Alfvén wave dissipation, Alfvén 1947; Heyvaerts and Priest 1983; van Ballegooijen et al. 2011). The corona is the outermost part of the solar atmosphere which extends outwards for several solar radii. Its structure is dominantly sculptured by magnetic fields.

The solar structure also determines the solar rotational behaviour. While the Sun 


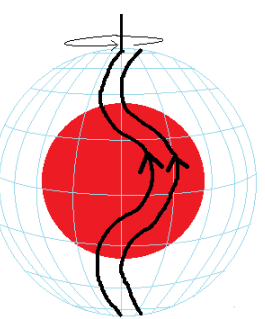

(a)

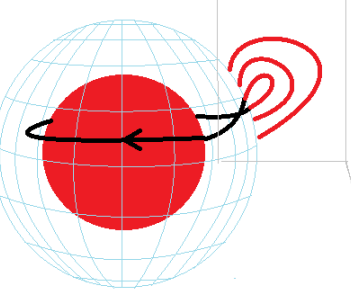

(d)

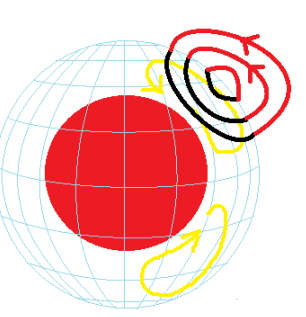

(g)

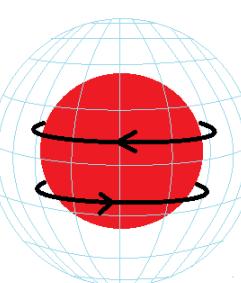

(b)

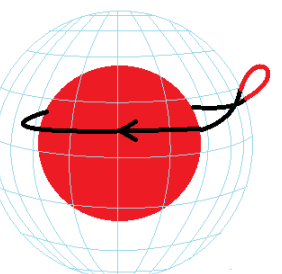

(c)
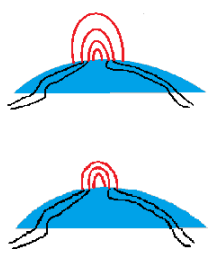

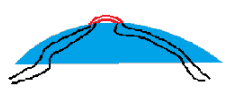

(e)

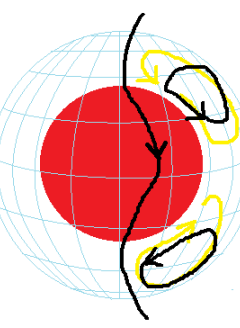

(h)

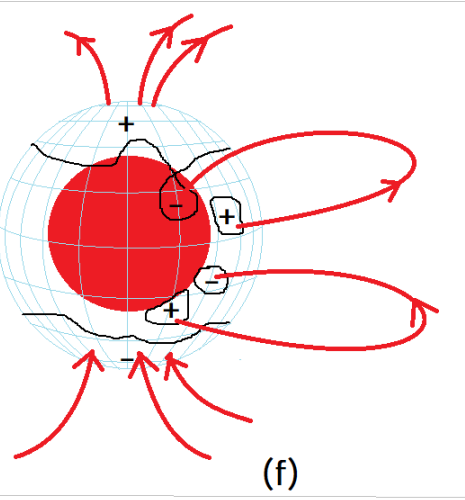

(f)

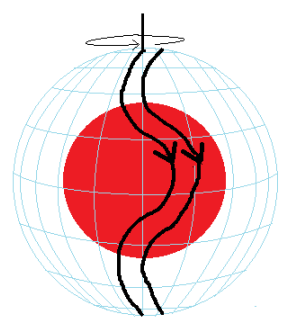

(i)

Figure 1.4: Schematic of the solar flux transport dynamo processes. Outer blue mesh represents the solar photosphere and the inner red sphere represents the radiative core, which rotates as a rigid body (see text for the detailed explanation of the process). Adapted from Dikpati and Gilman (2007).

rotates like a rigid body uniformly in the radiative interior, the solar rotation rate depends on latitude and depth in the convection zone (Goode 1995; Elsworth et al. 1995; Schou et al. 1998; Howe et al. 2000). This is the so-called differential rotation, which can also be observed in other astronomical objects, such as Jupiter, Saturn, other stars and galaxies. The transition layer between radiative zone and convection zone has a strong shear, and is called the tachocline (Spiegel and Zahn 1992). At the solar surface, the solar sidereal (with respect to the sky background) rotation has a period of $\approx 25$ days at the equator to $\approx 35$ days near the poles (e.g., Thompson et al. 1996). 


\subsubsection{Solar magnetic activity}

The solar magnetic field is responsible for many observable solar phenomena above the solar surface, such as sunspots, prominences, CMEs and flares. Although the exact solar dynamo mechanism is still unknown and debated, there is some degree of agreement and consensus between proposed models (for details, see review by Charbonneau 2010). The solar magnetic field is believed to be generated at the bottom of the convection zone (possibly near the tachocline, an interface layer between two zones) and is sustained through the solar cycle dynamo. The proposed surface flux transport model has found to successfully reproduce certain main surface magnetic features throughout the 11-year solar cycle, as illustrated in Fig. 1.4.

As mentioned, the radiative zone rotates as a rigid body while the convection zone rotates with varying velocities in latitude and radius. This strong shear produces a toroidal field from the poloidal field ( $\Omega$-effect), shown in Figs. 1.4a $-1.4 \mathrm{~b}$. Due to the enhanced buoyancy of the plasma, the magnetic fields start twisting while rising to the surface from the bottom of the convective zone (Babcock-Leighton mechanism). As the twisted loops emerge at the photosphere, the foot points of these loops form sunspots with opposite polarities ${ }^{1}$. The sunspots have tilt angles due to the Coriolis force (Joy's law), as shown in Figs. $1.4 \mathrm{c}-1.4 \mathrm{f}$ ( $\alpha$-effect). Figures $1.4 \mathrm{~g}-1.4 \mathrm{i}$ illustrate the meridional flows in the convection zone (a flow toward the poles at the surface and toward the equator below the surface as shown with yellow circles in the figure) bringing the decayed products of active regions polewards. The magnetic fields follow the meridional flow to the bottom of the convection zone and eventually form a poloidal field with an opposite magnetic orientation compared to the beginning of the process. This process takes roughly between $8-14$ years (on average 11.2 years) and therefore is also known as the 11-year solar cycle. The model successfully reproduces many features of the solar magnetic cycle (e.g., Babcock 1961; Leighton 1969; Dikpati and Gilman 2007).

The magnetic fields that are strengthened through the solar dynamo mechanism leave the location of the dynamo at the bottom of the convection zone, emerge to the solar surface and form various observable magnetic structures at the solar surface. These structures are observed with a wide range of magnetic intensity, morphology, lifetime and size. Largely, the magnetic structures are categorized into three regions: the active regions $(A R)$, the ephemeral regions (ER), and the quiet Sun region $(Q S)$ :

- Active regions (AR): Sunspots are the most prominent features of active regions with a typical lifetime of days to weeks. They appear in a latitude range of $\pm 5^{\circ}- \pm 40^{\circ}$, which are called the activity belts. The magnetic fields emerge outwards from the photosphere and form magnetic bipoles on the solar surface (Parker 1979; Spruit and Roberts 1983; Fan et al. 1994). The foot points of sunspots have strong vertical magnetic fields of $\approx 3000-4000$ Gauss (Livingston 2002). These strong magnetic fields suppress the convection underneath the solar surface, which results in lower temperatures $(\approx 4500 \mathrm{~K})$ of the sunspots compared to the surrounding photosphere (quiet Sun $\approx 5780 \mathrm{~K}$, Rempel and Schlichenmaier 2011; Solanki 2003). Hence, the central regions of the concentrated magnetic fields (umbra) have a brightness of

\footnotetext{
${ }^{1}$ The orientation of two sunspot bipoles in the same hemisphere within the same solar cycle is constant but is opposite to the other hemisphere. The orientation also alternates from cycle to cycle. This is called the Hale's polarity law (Hale and Nicholson 1925).
} 


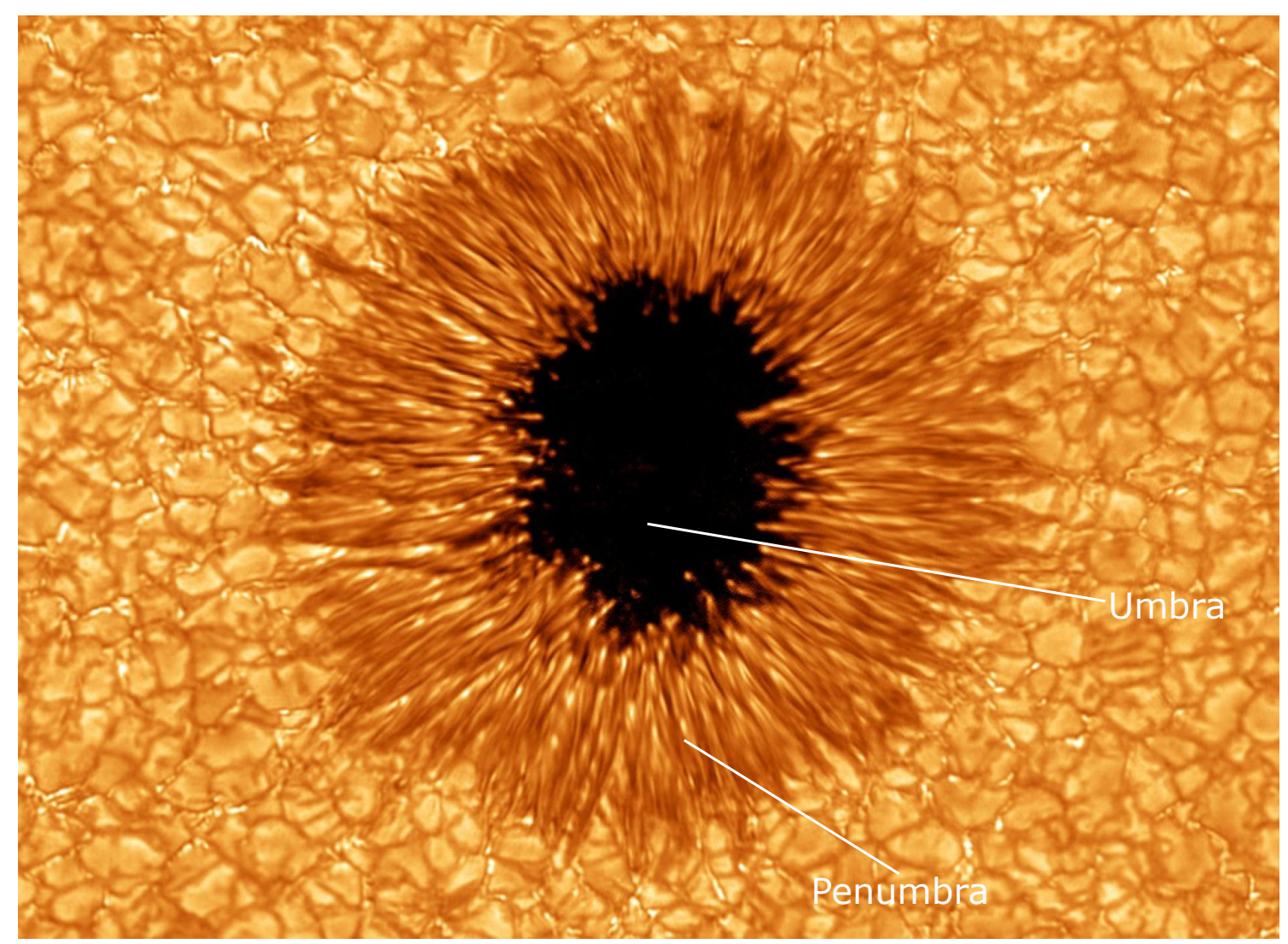

Figure 1.5: High resolution image of a sunspot (umbra and penumbra) observed by the New Solar Telescope installed at the Big Bear Solar Observatory, California. Courtesy of BBSO / NJIT

$10-30 \%$ of the quiet Sun (Mathew et al. 2007). The penumbra is the extended region around the umbra, which has more inclined (i.e., more nearly horizontal) magnetic fields, and appears less dark than the umbra (75-85\% of quiet Sun brightness). Penumbrae typically have temperatures of about $5400 \mathrm{~K}$ with magnetic field strengths of 1000 - 2000 Gauss (Solanki 2003). The energy blocked by sunspots is re-distributed in the convection zone owing to the heat conductivity of the solar plasma (Spruit 2000). Sunspots have average sizes of up to $\approx 50-60 \mathrm{Mm}$ in diameter. A highly spatially-resolved image of a sunspot with clear umbra and penumbra areas is shown in Figure 1.5.

Faculae are another typical magnetic feature associated with active regions and accompanying sunspots. Faculae are composed of highly magnetically concentrated features with a similar magnetic structure as in sunspots but smaller in size (a single magnetic element is from a few to tens of kilometers to a few hundred kilometers in diameter, Meunier 2003; Lagg et al. 2010). Although individual small bright magnetic elements are difficult to perceive, a group of the elements can be easily observed. Recently observations ${ }^{2}$ from the SUNRISE balloon-borne solar obser-

\footnotetext{
${ }^{2}$ In 2009 and 2013, from Kiruna, Sweden to northern Canada.
} 


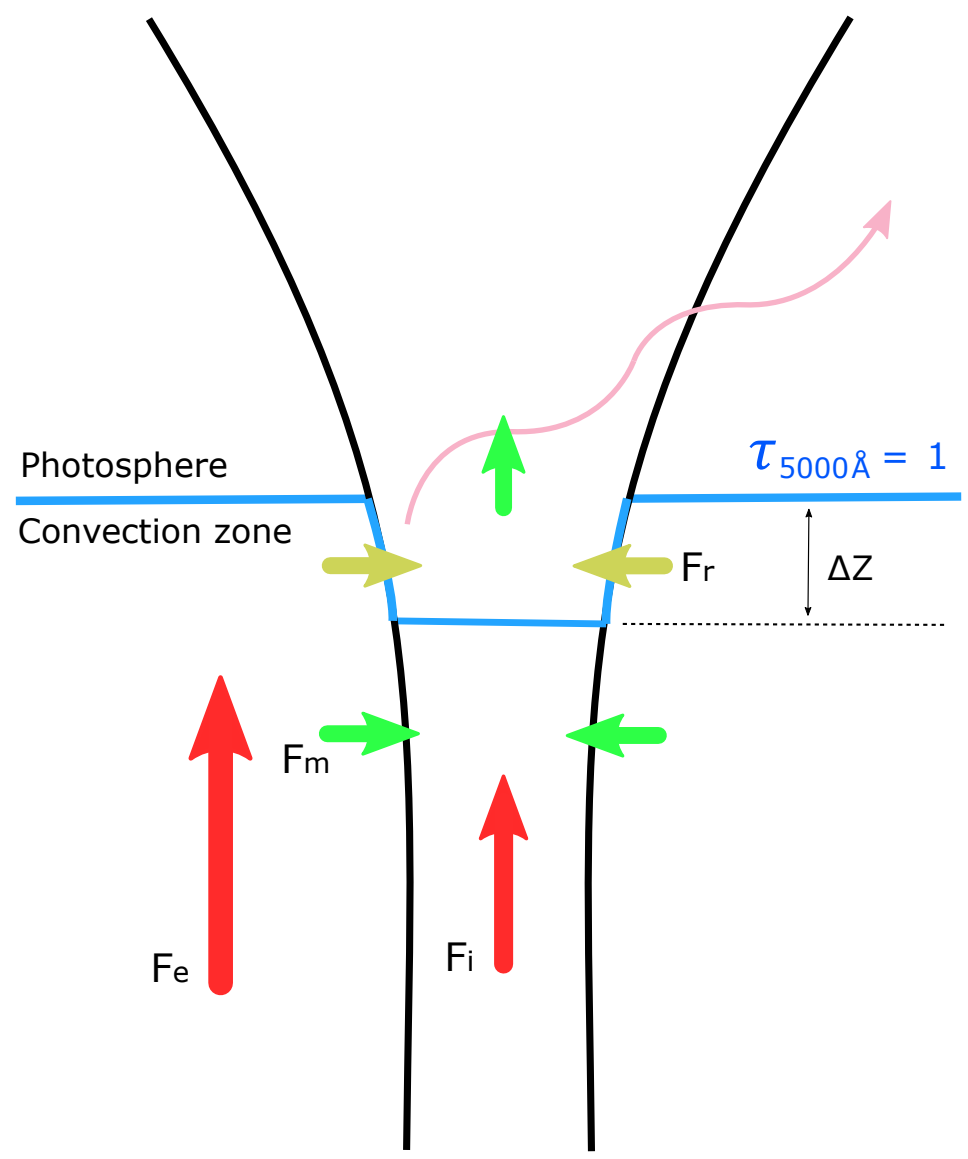

Figure 1.6: Sketch of a small-scale magnetic element which make up network and faculae. The magnetic flux tube is within the two black curves. The blue boundary shows the same level of optical depth $\left(\tau_{5000 \AA}=1\right)$, where below is the convection zone and above is the photosphere. $\Delta Z$ represents the Wilson depression due to the strong magnetic field inside the tube. The convective and radiative energy coming from below the solar surface are represented by red arrows (subscripts $i$ for inside the tube, and $e$ for the external surrounding). The radiation emitted through the walls of the flux tube (hot wall) is represented by the yellow arrows. Green arrows represent the mechanical energy flux excited by the turbulent motions in the convective zone. Reproduced after Solanki et al. (2013). 
vatory were able to detect and resolve the fine structures and the magnetic fields with a very high spatial resolution (under $100 \mathrm{~km}$ ) on the solar surface (Solanki et al. 2010, 2017; Lagg et al. 2010). The total surface coverage of faculae is an order of magnitude larger than that of sunspots (Solanki 1999). The ratio between facular area and sunspot area varies from 10 - 30 during solar maxima to $30-50$ during solar minima (Chapman et al. 1997; Fligge et al. 1998). The structure of a small-scale magnetic element is illustrated in Fig. 1.6. The blue line indicates the optical depth unity surface (solar surface, $\tau_{5000 \AA}=1$ ) and the black curves in the middle illustrate the magnetic flux tube boundaries. Because of the strong magnetic field inside the flux tube, the convection inside it is suppressed. The strong field produces magnetic pressure, which balances with the surrounding gas, and causes the depression of the optical depth unity surface (Wilson depression). This allows us to see deeper in the flux tube (indicated by depth $\Delta Z$ ) below the surface. The energy is transported upwards (red arrows) in the convection zone and radiates into the optically thin flux tube (yellow arrows) from the walls of the tube and heats up the interior of the tube (therefore the name "hot wall"). The escaping photons from the hot walls can be easily observed, especially when the structure is located near the limb (Spruit 1976; Carlsson et al. 2004; Keller et al. 2004). Moreover, the turbulent movements in the surrounding convective zone can shake the magnetic tube and excite mechanical waves. The mechanical energy propagates upwards to the upper photosphere and the chromosphere, where it can dissipate and heat the local gas and cause the strong emission in UV wavelengths and in the $\mathrm{Ca}$ II $\mathrm{H}$, K lines (Rezaei et al. 2007; Schrijver et al. 1989). The latitudinal distribution of faculae is about $15^{\circ}$ broader compared to the sunspots.

- Ephemeral regions (ER): Ephemeral regions are small and short-lived bipolar regions on the solar surface distributed widely in latitudes. Figure 1.7 shows a full-disc magnetogram of the Sun. Black and white regions represent the negative (inwards) and positive (outwards) magnetic polarities, respectively. The white box encloses an active region which is enlarged in two smaller flanking panels (upper: magnetogram; lower: continuum image). A comprehensive work done by Harvey over more than one decade (between the end of the cycle 20 to the beginning of the cycle 22) reveals many detailed physical characteristics of ERs. Their lifetimes are typically of hours, and their diameters are a few to tens of Megameters (Harvey and Martin 1973; Title 2000; Hagenaar 2001). The total magnetic flux of ERs has a wide range between $10^{17}-6.7 \times 10^{19} \mathrm{Mx}$ with an average value found to lie in the range between $9.3 \times 10^{18}-3 \times 10^{19} \mathrm{Mx}$ (Harvey and Martin 1973; Martin and Harvey 1979; Zwaan 1985; Harvey 1992; Hagenaar 2001; Hagenaar et al. 2008; Wang et al. 2012; Zhao and Li 2012; Yang and Zhang 2014). A relationship between the ER cycle and the corresponding AR cycle has also been found. For instance, the amplitude of the ER cycle can be described linearly proportional to that of the AR cycle (Martin and Harvey 1979). Furthermore, Martin and Harvey (1979) and Harvey (1993, 1994) have also found that the ER cycles tend to appear a few years (typically 2 3 years) earlier and last longer than the corresponding AR cycles. Therefore, the 


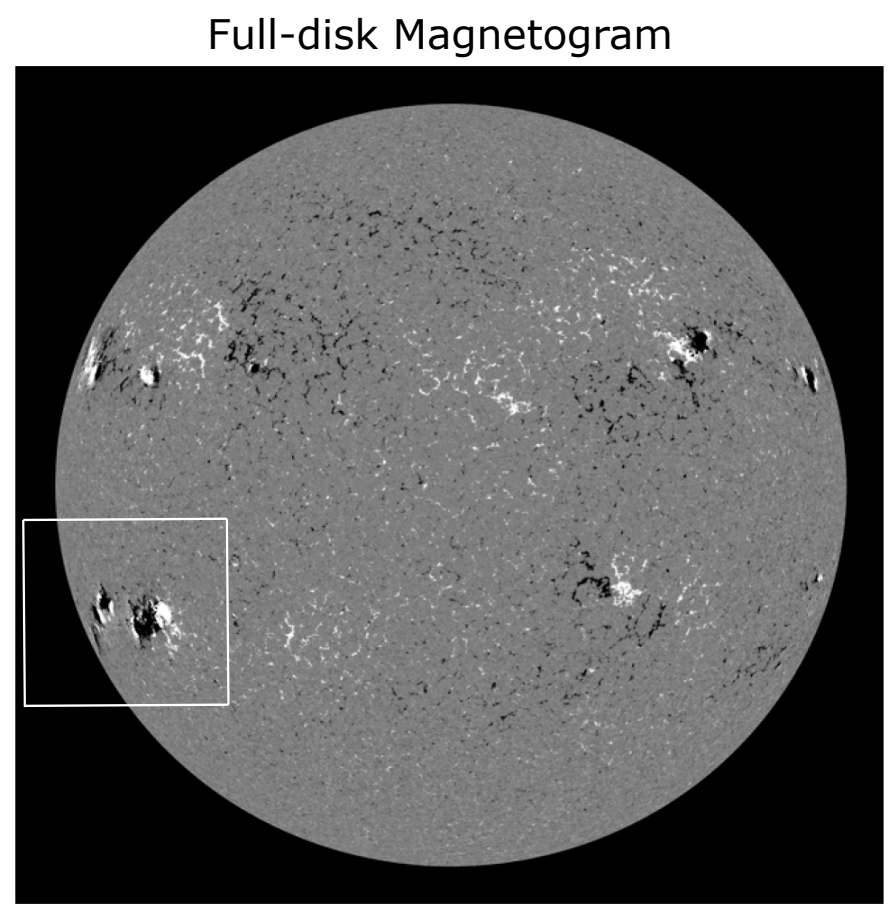

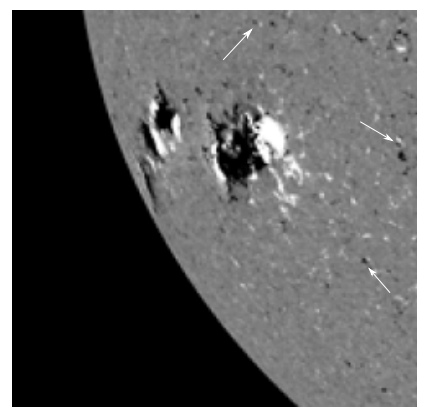

Magnetogram

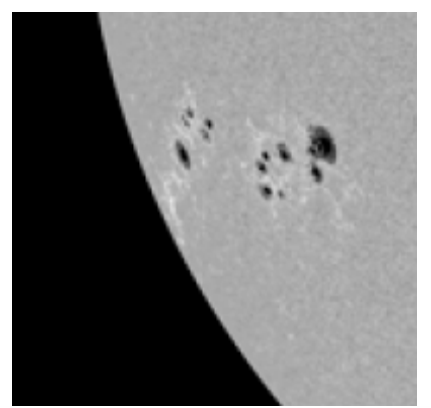

Continuum intensity

Figure 1.7: Full disc magnetogram of the Sun on 30th May. 2011. The black and white regions represent the negative (into the surface) and positive (out of the surface) magnetic polarities, respectively. An active region is framed by a white box and is enlarged in two flanking panels (upper: magnetogram, lower: continuum image). Small ephemeral regions are indicated by the white arrows. Adapted from Yeo et al. (2013).

ER cycles from two consecutive cycles overlap each other during the solar activity minima. This overlap of ER cycles is possibly responsible for the secular change in the photospheric magnetic field and the irradiance (Solanki et al. 2000, 2002b).

- Quiet Sun $(Q S)$ : The regions free of, or with only weak magnetic field, are called the quiet Sun. The quiet Sun is mostly covered by granules, which are overturning convective cells. Granules were first observed and described by Herschel (1801). The hot plasma in the convection zone rises to the solar surface at the centre of a cell, then cools down and sinks back to the convection zone at the cell boundaries (e.g., Nordlund et al. 2009). As a result, the boundaries of the cells appear darker. Carried by the convective motions, the magnetic field aggregates in the intergranular lanes (the boundaries between granules). The sizes of granules are usually about a few thousand kilometres. The granules are highly dynamic and evolve on time scales of about 5-10 minutes. They appear and disappear instantaneously due to the convective motion of the plasma and appear in various sizes and irregular shapes. A granular pattern in the quiet Sun region observed by the Swedish Solar Telescope at a high spatial resolution (Scharmer et al. 2003) is shown in Fig. 1.8. 


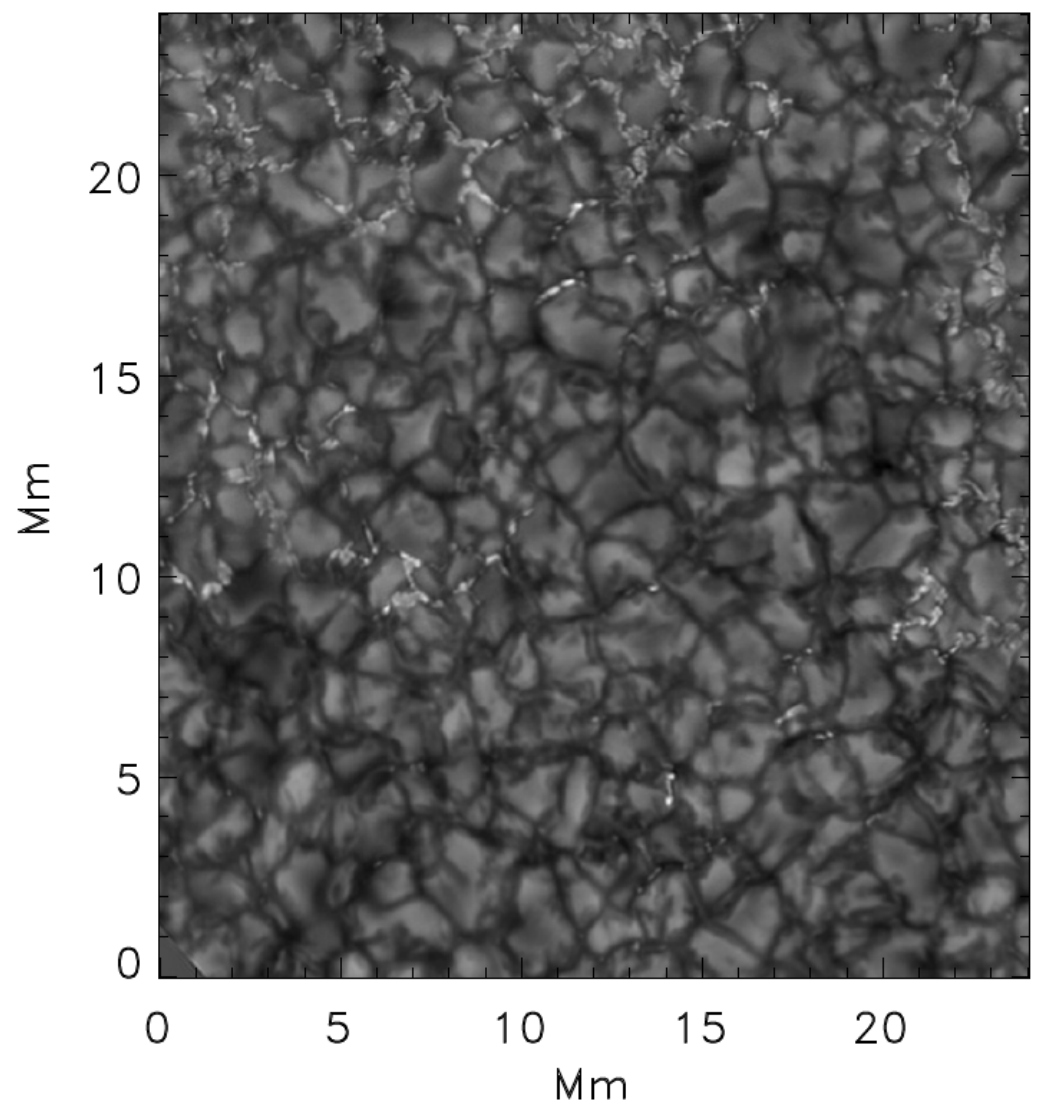

Figure 1.8: Image of granulation pattern in the G-continuum. Taken from the Swedish 1-m Solar Telescope and Institute of Theoretical Astrophysics, Oslo. 


\subsubsection{Solar cycles}

Through his observations of the number of sunspot, Heinrich Schwabe discovered the socalled 11-year solar cycle in 1843 (Schwabe 1844). Later, it became clear that apart from the sunspot number, other solar observables also vary over the course of the solar cycle. For instance, the emergence latitudes of sunspots vary with the phase of the 11-year solar cycle. The sunspots typically emerge at around $\pm 30^{\circ}- \pm 40^{\circ}$ latitude at the beginning of a cycle and migrate to $\pm 5^{\circ}$ latitude at the end of a solar cycle (Spörer Law of Zones, Maunder 1903). The migrating pattern forms the well-known butterfly diagram.

Herschel (1801) argued that the Sun is darker during the solar maxima due to the presence of more sunspots and the radiative output is therefore reduced. Today, this hypothesis has been proven wrong, and the TSI is found to vary in-phase with the 11-year solar cycle (Sect. 1.3.1). The increase of the TSI during solar maxima is due to the dominating presence of the bright faculae, which accompany sunspots and overcompensate the sunspot darkening. Moreover, the solar magnetic field is weaker during solar minima while it is stronger during solar maxima (Chap. 4). Some other solar phenomena, such as solar flares, prominence eruptions and CMEs also occur more frequently around the solar maximum periods than during solar minimum periods.

Aside from the 11-year solar cycle, solar variation with longer periodicities have also been noticed. For example, a longer trend with a periodicity of $\approx 90$ years (Gleissberg cycle, Gleissberg 1939, 1960; Garcia and Mouradian 1998; Feynman and Ruzmaikin 2014; Vázquez et al. 2016) is also observed in the sunspot number record. Others, such as the De Vries (or Suess) cycle with a periodicity of $\approx 200$ years (Suess 1980), an unnamed cycle with a periodicity of $\approx 600-700$-years cycle (Vitinskij et al. 1986; Sonett and Fin-

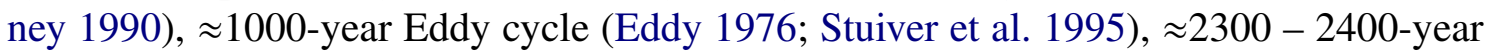
Hallstatt cycle (Stuiver et al. 1991; Damon and Sonett 1991; Damon and Jirikowic 1992; Vasiliev and Dergachev 2002) and an even longer unnamed 6000-year cycle (Xapsos and Burke 2009) have also been claimed to be present in the long-term cosmogenic isotope records. In particular, the 6000-year cycle is still doubtful since the cosmogenic isotope data covers only about 11000 years.

By analysing the isotope-based $\mathrm{SN}$ reconstruction, the solar activity has been found to show many extended active and quiet periods in the past. The strongest of these are called grand maxima and grand minima, respectively. Commonly, a grand maximum (grand minimum) is defined as the a period when the sunspot numbers are higher than 50 (lower than 15) for at least two consecutive decades (Usoskin et al. 2007). There have been at least 20 grand maxima and 27 grand minima identified over the Holocene ${ }^{3}$ (e.g., Usoskin et al. 2007, 2016c; Inceoglu et al. 2015). Over the last millennium, there are five grand minima confirmed: the Oort minimum $(1010$ - 1050 AD), the Wolf minimum (1280 1340 AD), the Spörer minimum (1460 - 1550 AD), the Maunder minimum (1645 - 1715 $\mathrm{AD})$, and the Dalton minimum (1790 - $1830 \mathrm{AD})$.

Studies have shown the grand minima tend to occur in clusters within $2000-3000$ years while the occurrence rate of grand maxima follows an exponential distribution (Usoskin et al. 2007). The Sun may spend as much as $\approx 16-33 \%$ of its time in grand minima (Usoskin et al. 2016a) and up to $12 \%$ of the time in a state of high activity (Usoskin et al. 2006a; Usoskin 2017). During the last 7 decades, the solar activity has been re-

\footnotetext{
${ }^{3}$ Approximately 11700 years after the last ice age till now.
} 
ported to be in an unusually high state (Usoskin et al. 2003; Solanki et al. 2004), with a doubled average sunspot number between 1950 - 2000 compared to that between 1750 - 1900. The recent active period has been regarded as a modern grand maximum (Zięba and Nieckarz 2014; Usoskin 2017) while the observed sunspot numbers in the last two cycles (\#23 and \#24) started to decrease and the solar activity has returned to a moderate state. Although some authors have proposed that the Sun is entering the next Maunder minimum-like state (e.g., Zolotova and Ponyavin 2014; Zharkova et al. 2015; Zachilas and Gkana 2015), it is important to note that our ability of predicting solar activity is still very limited and has large uncertainties (Cameron et al. 2013). Statistically, after a period of moderate activity following a grand maximum, the next grand extremum could be a grand maximum as likely to be a grand minimum (Solanki and Krivova 2011).

\subsection{Solar total and spectral irradiance}

For a long period of time, the radiative output of the Sun was thought to be invariant, and therefore termed the "solar constant". Even though the relationship between the solar energy output and the solar magnetic activity was speculated long before the direct measurement of the solar irradiance (Abbot 1923; Smith and Gottlieb 1975; Eddy 1976), it was impossible to accurately measure the solar spectral irradiance by ground-based instruments due to the atmospheric interference. Solar radiometers onboard of many satellites (Sect. 1.3.1) revealed the fact that indeed the solar energy output is not only varying at all time scales but also correlated strongly with the solar magnetic activity.

The Sun is continuously radiating energy outwards. This energy is a function of wavelength and is termed the solar irradiance. Total solar irradiance (TSI) is the spectrallyintegrated energy flux per unit area that is normalized to $1 \mathrm{AU}$ (annual mean distance between the Sun and the Earth; $149597870 \mathrm{~km}$ ), while the spectrally-resolved irradiance is termed solar spectral irradiance (SSI).

The TSI is primarily dominated by the visible and infrared (IR) bands of the spectrum (which contribute $\approx 39 \%$ and $\approx 53 \%$ of the TSI, respectively) and about $30 \%$ of the TSI comes from the wavelengths longwards of $1000 \mathrm{~nm}$ (Woods et al. 2009). The TSI varies at a level of $\approx 0.1 \%$ on 11 -year solar cycle time scale and can sometimes reach $0.3 \%$ on the solar rotation time scale because of the passage of the bright/dark features (Ermolli et al. 2013). While the ultraviolet (UV) contribution to the TSI is small $(<8 \%)$, it contributes more than 50\% of the TSI variability (e.g., Floyd et al. 2003; Krivova et al. 2006). In the following, we will give a brief review of the history of measuring the solar irradiance, its origins, and the models.

\subsubsection{Measurements}

\subsubsection{Measurements of TSI}

Before the satellite era, the solar irradiance was measured as a constant value with a wide range from $1338 \mathrm{~W} / \mathrm{m}^{2}$ to $1428 \mathrm{~W} / \mathrm{m}^{2}$ (see reviews by Smith and Gottlieb 1974; Froehlich and Brusa 1981). The TSI measurements were so inaccurate that the subtle changes in the solar irradiance could not be detected. 


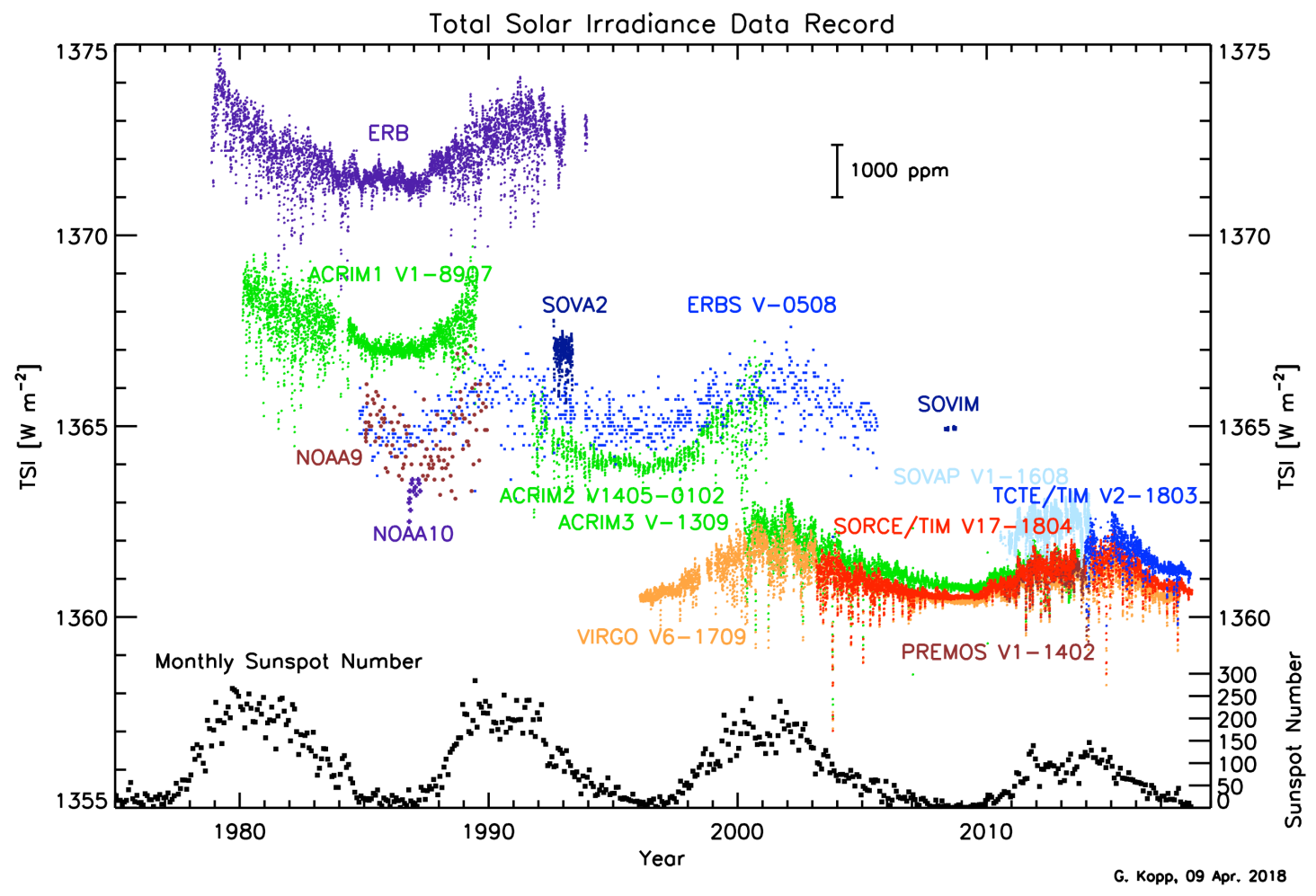

Figure 1.9: Space-based TSI measurements covering the period of 1978 - 2018. Individual measurements of different satellites are shown by different colours (labelled in the plot). The monthly mean sunspot numbers are represented by dots in the bottom part of the plot. Courtesy of G. Kopp ${ }^{4}$.

Direct measurements of TSI by space-based instruments began with a series of satellites missions in 1978 (Willson et al. 1981; Rottman 1988; Floyd et al. 2003; Fröhlich 2012; Kopp 2014). Fig. 1.9 summarizes the space-based TSI measurements over time, with different colours indicating different satellites. The corresponding sunspot number is plotted at the bottom. It is noticeable that all the measurements share similar traits in the solar irradiance, such as the presence of the 11-year solar cycle and the short-term peaks and dips due to the passage of surface magnetic features across the solar disc (Willson et al. 1981; Hudson et al. 1982; Foukal and Lean 1986). However, it is obvious that the absolute levels of these measurements (especially at early times) do not match each other. The TSI values measured by the SORCE/TIM ${ }^{5}$ are $\approx 5 \mathrm{~W} / \mathrm{m}^{2}$ lower than other the measurements by other contemporaneous instruments, which themselves disagree by a few $\mathrm{W} / \mathrm{m}^{2}$.

These differences came from the optical (aperture) design. It has been demonstrated by the TSI radiometer Facility (TRF, Kopp et al. 2007) that the other instruments (e.g., ACRIMSAT/ACRIM ${ }^{6}$ and $\mathrm{SoHO} / \mathrm{VIRGO}^{7}$ ) had a different arrangement of two apertures

\footnotetext{
${ }^{4}$ http: //spot . colorado. edu/ koppg/TSI/

${ }^{5}$ Total Irradiance Monitor onboard the SOlar Radiation and Climate Experiment satellite.

${ }^{6}$ Active Cavity Radiometer Irradiance Monitor onboard the Active Cavity Radiometer Irradiance Monitor SATellite.

${ }^{7}$ Variability of SOlar Irradiance and Gravity Oscillations onboard the Solar Heliospheric Observatory.
} 
(view-limiting and precision aperture), which allowed radiation to be deflected inside the cavity (Kopp et al. 2007; Fröhlich 2012). The optical design of TIM has successfully reduced the effects of scattered light and diffraction in the instrument. Following this, the measurements from other instruments have been calibrated and adjusted downwards to the TIM measurements. This lower level of TSI measurements has been validated by the recently launched Picard/PREMOS ${ }^{8}$ instrument (Schmutz et al. 2009, 2013). It was the only TSI radiometer, which was calibrated in vacuum at full TSI power levels prior to its launch. Therefore, the lower TSI level measured by the SORCE/TIM is likely accurate. In this thesis, we take the value of $1360.52 \mathrm{~W} / \mathrm{m}^{2}$ for the 2008 minimum (averaged over the period November 2008 to January 2009) based on the SORCE/TIM measurement (Kopp and Lawrence 2005; Kopp et al. 2005a,b).

Due to the limited lifetime of the instruments, all the measurements lasted not longer than one full solar cycle, which makes it difficult to assess the long-term secular change. Due to the change in the instrumental sensitivity and their degradation over time, it is not trivial to calibrate all the measured records and to determine the long-term solar variability. To meet this need, three composites were, therefore, constructed: (1) PMOD (Fröhlich 2000, 2003, 2006, 2009) (2) IRMB (also called RMIB, Dewitte et al. 2004), and (3) ACRIM (Willson and Hudson 1991; Willson 1997; Willson and Mordvinov 2003), as shown in Fig. 1.10.

These three different composites agree with each other well in short-term changes while the difference in the long-term trend (see Fig. 2 in Solanki et al. 2013) is more critical, especially in many climate studies. For instance, the PMOD composite shows a continuous increasing trend from the solar activity minimum in 1986 to the $2008 \mathrm{mi}-$ nimum. The ACRIM composite shows an increasing trend from the solar minimum in 1986 to the 1996 minimum and a decreasing trend from the solar minimum in 1996 to the 2008 minimum. The IRMB composite shows a continuous increasing trend from the solar minimum in 1986 to the 2008 minimum (opposite to the PMOD). A large part of the difference in the long-term trend among the three TSI composites is due to the correction of the early series measured by Hickey-Frieden (HF) radiometer onboard Nimbus-7/ERB ${ }^{9}$ (Fröhlich 2006, 2012). Only PMOD composite record applies such correction while both ACRIM and IRMB composites do not.

\subsubsection{Measurements of SSI}

The SSI has been observed by various space-borne instruments over the last four decades. Each instrument covers different observation periods and spectral ranges, as summarized in Fig. 1.11. The early measurements of the SSI were limited to the UV wavelength range below $400 \mathrm{~nm}$. As with TSI (Sect. 1.3.1.1), UV radiation $(120-400 \mathrm{~nm})$ was monitored almost without interruption since 1978, started with the measurement by

\footnotetext{
${ }^{8}$ Precision Monitor Sensor on board the Picard satellite.

${ }^{9}$ Earth Radiation Budget instrument onboard the Nimbus-7 satellite
} 


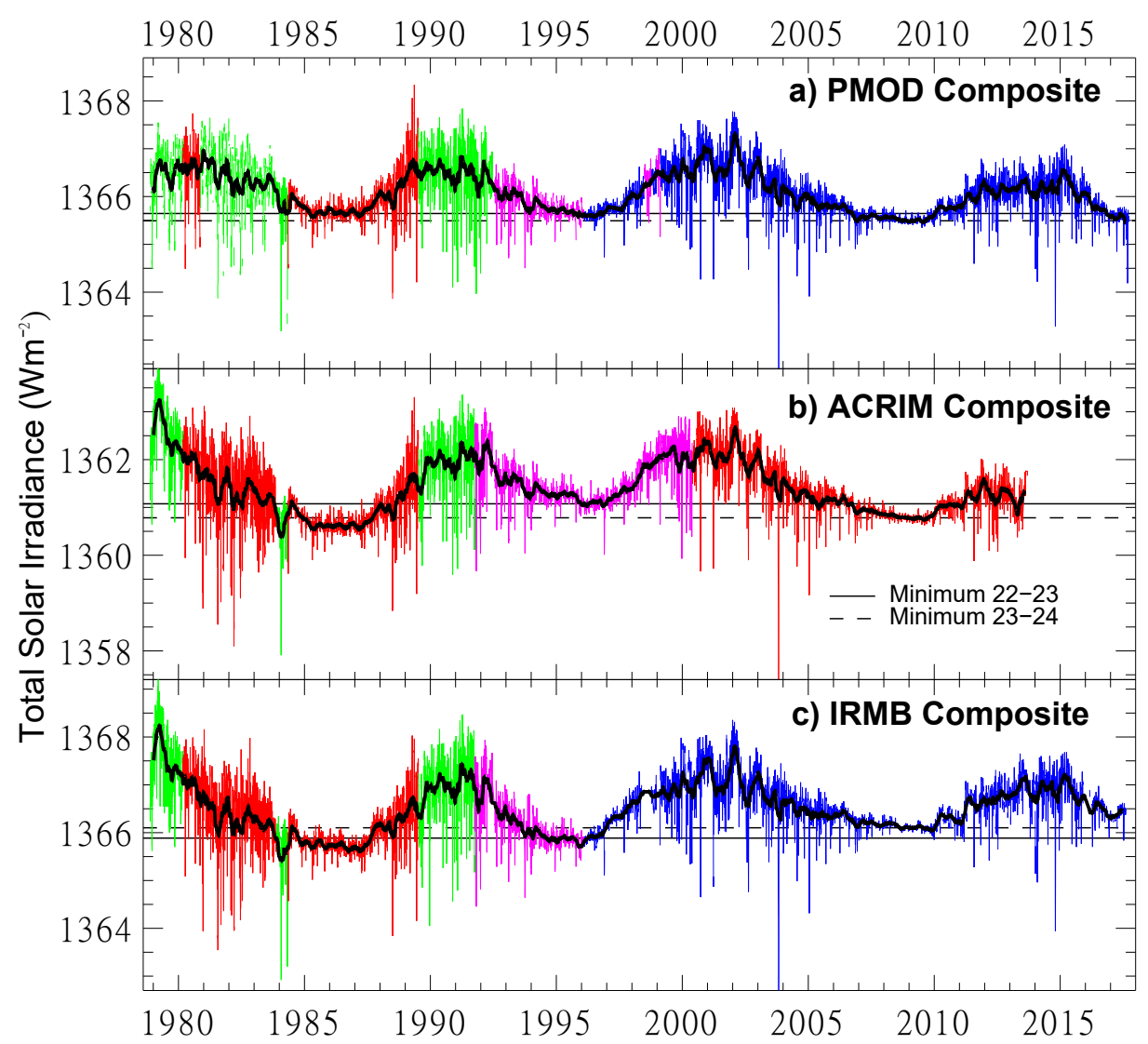

Figure 1.10: Three composite records of TSI since 1978: (a) PMOD, (b) ACRIM, (c) IRMB. Courtesy of PMOD/C. Fröhlich ${ }^{10}$.

Nimbus-7/SBUV ${ }^{11}$ (Cebula et al. 1992). Since 1991, UARS/SOLSTICE ${ }^{12}$ (Rottman et al. 1993) and UARS/SUSIM ${ }^{13}$ (Brueckner et al. 1993) provided the two main UV observation records (e.g., Floyd et al. 2003). Later, ERS-2/GOME ${ }^{14}$ (launched in 1996; Weber et al. 1998; Munro et al. 2006) and ENVISAT/SCIAMACHY ${ }^{15}$ (launched in 2002; Skupin et al. 2005) started to measure a wider range of solar spectrum, in the $240-790 \mathrm{~nm}$ and $240-2380 \mathrm{~nm}$, respectively. However, these two instruments were focused on atmospheric sounding and lacked of in-flight degradation tracking, making them unsuitable for the study of solar SSI variation. With the launch of the SORCE satellite in 2003, a broad spectral range of SSI (from Lyman- $\alpha$ to $2400 \mathrm{~nm}$ ) with a high temporal resolution was available since 2004. The regular measurements were made by two instruments: the SORCE/SOLSTICE (Snow et al. 2005) and the SORCE/SIM (Harder et al. 2005, 2009).

Consistent phenomena have been observed among the SSI measurements, revealing that the relative SSI variation is strongly wavelength-dependent and increases towards shorter wavelengths (Fig. 3 in Solanki et al. 2013). The visible and IR ranges have the

\footnotetext{
${ }^{10}$ https://www . pmodwrc.ch/en/research-development/solar-physics/tsi-composite/

${ }^{11}$ Solar Backscatter Ultraviolet Radiometer.

${ }^{12}$ Solar Stellar Irradiance Comparison Experiment onboard the Upper Atmosphere Research Satellite.

${ }^{13}$ Solar Ultraviolet Spectral Irradiance Monitor.

${ }^{14}$ Global Ozone Monitoring Experiment onboard the second European Remote Sensing satellite.

${ }^{15}$ SCanning Imaging Absoption spectroMeter for Atmospheric CHartographY on board the ENVironmental SATellite.
} 


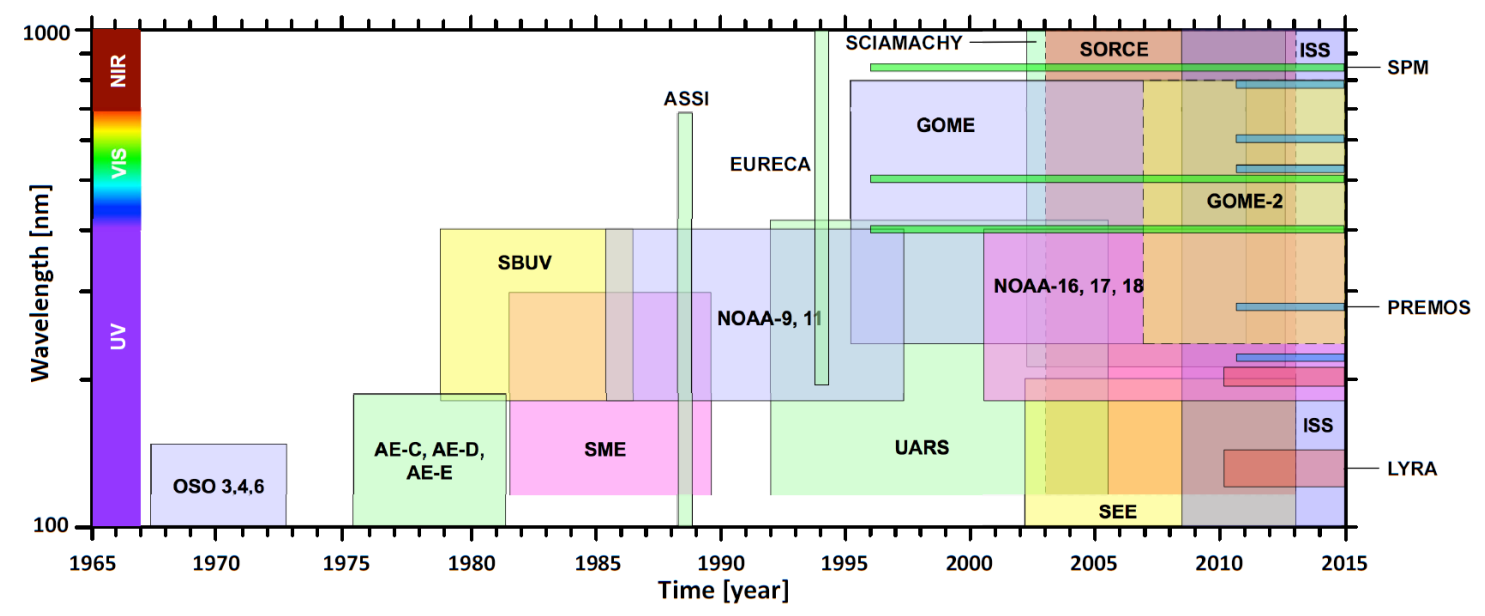

Figure 1.11: Timeline (X-axis) and spectral range (Y-axis, above $100 \mathrm{~nm}$ ) of the SSI observations since 1965. Taken from Ermolli et al. (2013).

least variability $(\approx 0.1 \%)$, whereas the variability of $1 \%$ to $100 \%$ are observed in the UV wavelengths (e.g., Floyd et al. 2003). For extent details of the SSI measurements, we refer to the reviews by Domingo et al. (2009); Fröhlich (2012); Ermolli et al. (2013); Solanki et al. (2013) and Yeo et al. (2014a).

\subsubsection{Physical mechanisms of solar irradiance}

From the space-borne measurements with high temporal and spatial resolutions, it is seen that the solar irradiance varies at all time scales, driven by a range of physical processes. The acoustic oscillations excited by the turbulent motions in the convection zone is a significant source of the irradiance variation around 5 minutes ( $p$-modes, Gough and Toomre 1991; Christensen-Dalsgaard 2002). The granulation is responsible for the variation between minutes to hours (Hudson 1988; Gough and Toomre 1991; Seleznyov et al. 2011; Shapiro et al. 2017).

On time scales longer than one day to decades, solar irradiance variability is modulated mainly by the photospheric magnetic activity, namely, the evolution process and the spatial distribution of the solar surface magnetism. While sunspots have typical life times of a few weeks, faculae usually live much longer. Additionally, as introduced in Sect. 1.2.2, the ERs are the main contributor to the secular change due to the overlap of two consecutive ER cycles. As a consequence, the changes of solar irradiance on time scale longer than one day could be contributed mainly by the summed effects of the darkening and brightening caused by solar surface magnetic features.

The thermal relaxation of the convection zone dominates the variation on time scale of about $10^{5}$ years (Spruit 2000). On time scale longer than $10^{6}$ years, the variation of the solar irradiance results from the changes in the chemical composition of the Sun's core (Sackmann et al. 1993; Charbonnel et al. 1999; Mowlavi et al. 2012). In this thesis, we study the solar irradiance variability on time scale of centuries and millennia. The changes caused by the oscillations, granulation and the long-term chemical evolution in the core are not considered. 


\subsubsection{Models of irradiance variability}

The satellite measurements of solar irradiance, as introduced in the previous sections, cover only a limited period of time ( $\approx$ four decades), with significant uncertainty and instrumental degradations. Therefore, modelling the solar irradiance and its variability can assist us not only to better understand the physical processes of the solar irradiance variability, but also to reconstruct the solar variability in the past.

Since we are focussing on the solar irradiance variability on time scales of days to millennia, which is thought to be caused mainly by the surface magnetic variability (Sect. 1.3.2), the present-day models that employ such an assumption (namely that the solar variability on time scales longer than a day is driven by the distribution and the evolution of the solar surface magnetism) have been the most successful in reproducing the observed solar irradiance variability. Therefore, knowledge of the evolution of the solar surface magnetic fields is required for the models on these time scales.

Various models use different proxies, or their combinations, to describe the effects of either the sunspot darkening or the facular brightening. In Sect. 1.3.3.1 we will give an overview of the proxies used by different models. Next, two classes of currently existing TSI/SSI models, "proxy" and "semi-empirical" models, are described in Sect. 1.3.3.2 and 1.3.3.3, respectively.

\subsubsection{Proxies}

By investigating the early TSI measurements, Willson et al. (1981), Hudson et al. (1982) and Foukal and Lean (1986) have found that certain apparent peaks and dips shown in the TSI records coincided with the passage of sunspots and faculae. Therefore, with the development of modelling the solar irradiance, indices of surface bright and dark features became crucial ingredients.

The dark (sunspots and pores) and the bright (faculae and networks) features can be represented by various relevant observable quantities. The influence of darkening is usually represented by the sunspot number or the photometric sunspot index (PSI, Hudson et al. 1982; Froehlich et al. 1994). The brightening is typically represented by the chromospheric indices, such as the F10.7 radio flux (Tapping 1987, 2013), Mg II (Heath and Schlesinger 1986) and Ca II K-line (Keil et al. 1998). Others, such as full-disc intensity images and magnetograms, can also help to determine the surface coverages and the brightening/darkening effects.

Potentially, full-disc Ca II K spectroheliograms could be useful for determining the plage $^{16}$ regions in the past (Skumanich et al. 1975; Schrijver et al. 1989; Loukitcheva et al. 2009), as they have been recorded regularly since the beginning of the 20th century. However, these observations from many observatories around the globe suffer from various artefacts and problems, which make them difficult to employ in long-term solar variability studies. These issues are: lack of photographic calibration, undocumented instrumental changes and the decay of the photographic plates (Ermolli et al. 2009). An accurate processing and careful analysis of all available historical data are hence required. A comprehensive work, including developing an automatic processing and photometric

\footnotetext{
${ }^{16}$ The choromospheric regions corresponding to faculae.
} 
calibrations, correcting various artefacts and applying the central-to-limb variation (CLV), was performed by Chatzistergos et al. (2018) and is still in progress.

All the mentioned indices above are limited by the observational periods, and most of them are only available for few decades with reliable quality. Among them, group sunspot number is the only record that covers the entire four centuries (Hoyt and Schatten 1998). Nevertheless, mistakes and errors have been reported in the early period of this record. There are many proposed revised sunspot number series (e.g., Lockwood et al. 2014c; Cliver and Ling 2016; Svalgaard and Schatten 2016; Usoskin et al. 2016c; Chatzistergos et al. 2017), but none of these has been universally accepted so far. Consequently, the topic is still under debate (more details see Sect. 2.1).

To go even further back in time, indirect proxies of solar activity are needed. Concentration of the cosmogenic isotopes $\left({ }^{14} \mathrm{C},{ }^{10} \mathrm{Be}\right.$ and $\left.{ }^{36} \mathrm{Cl}\right)$ in terrestrial natural archives have been found to be associated with the solar magnetic activity (e.g., Stuiver 1961; Lal and Peters 1967; Eddy 1976; Stuiver and Quay 1980; Beer et al. 1988, and Sect. 2.4). This is because they are mainly produced in the terrestrial atmosphere by high-energy galactic cosmic rays, whose intensity is highly modulated by both the geomagnetic field and the solar magnetic field (e.g., Masarik and Beer 1999; Beer 2000a; Usoskin et al. 2002; Muscheler et al. 2004; Caballero-Lopez and Moraal 2004).

\subsubsection{Proxy models}

Proxy models use the said indices (Sect. 1.3.3.1) to describe the effects of the sunspot darkening and the facular brightening directly. The TSI and SSI variability reconstructed by this type of models are calculated using linear combination of these solar activity indices. The coefficients of the combination are obtained from the regression of the indices to the measured TSI/SSI (Hudson et al. 1982; Oster et al. 1982; Chapman and Boyden 1986; Foukal and Lean 1986; Chapman et al. 1996, 2012, 2013; Lean et al. 1997; Lean 2000; Preminger et al. 2002; Coddington et al. 2016; Yeo et al. 2017a).

In the proxy models, the UV variability is usually determined by fitting the index data to the rotational variability in the measurements. Therefore, such models have to rely on reliable measurements of solar irradiance, and are often limited by the availability of the measurements and the uncertainties in the index data. In addition, these models usually assume a linear indices-to-irradiance relationship at all time scales, which is not true for the chromospheric indices (Solanki and Krivova 2004; Foukal et al. 2011). As a consequence, reconstructing SSI by proxy models is not straightforward.

The two main current proxy models are the NRLTSI/NRLSSI ${ }^{17}$ (Lean et al. 1997; Lean 2000; Coddington et al. 2016) and the EMPIRE ${ }^{18}$ (Yeo et al. 2017a). As the names suggest, NRLTSI gives only the TSI, while NRLSSI provides only the spectral irradiance. In contrast, EMPIRE gives both, TSI and SSI. The NRLSSI reconstructions have been reported to provide a weaker solar cycle variation in the UV than the reconstructions with the EMPIRE model (Yeo et al. 2017a) and the semi-empirical model by Morrill et al. (2011). The difference between the NRLSSI and the EMPIRE is attributed to the different statistical approaches. While the NRLSSI model uses the ordinary least squares (OLS) regression, which assumes the uncertainties in the predictors are negligible, the

\footnotetext{
${ }^{17}$ Naval Research Laboratory Solar Spectral Irradiance.

${ }^{18}$ EMPirical Irradiance REconstruction.
} 
EMPIRE applies the orthogonal distance regression (ODR) regression, which takes those uncertainties into account.

On time scales longer than centuries, when the directly observed indices are not available, cosmogenic isotopes are used as indirect indicators (proxies) of solar activity. The isotope data are usually first converted into a Sun-related parameter, solar modulation potential ( $\phi$, Sect. 2.3.1). Steinhilber et al. $(2009,2012)$ and Delaygue and Bard (2011) used simple linear regressions between $\phi$ and the other solar quantities (e.g., TSI and open magnetic flux) to estimate the solar irradiance. However, these linear approaches limit the ability to calculate the spectrally-resolved irradiance and only the TSI reconstructions are available. Furthermore, there are various non-linear processes involved at multiple stages in the chain of processes, which eventually lead to the production of the cosmogenic isotopes (Sect. 2.3). The reconstructions based on linear estimation methods might therefore not be realistic and have large uncertainties.

\subsubsection{Semi-empirical models}

The second type of the TSI/SSI models, semi-empirical models, divide the solar surface into multiple features, termed "components". The filling factors (the coverages) and the time-evolution of these components in such models are determined from full-disc (spatially-resolved) observations (Fligge et al. 2000; Krivova et al. 2003; Ermolli et al. 2003, 2011; Fontenla and Harder 2005; Wenzler et al. 2004, 2005, 2006; Shapiro et al. 2010; Yeo et al. 2014b). Next, the time-independent intensity spectrum of each component is employed to calculate the solar irradiance at any given time. The intensity spectra are calculated using spectral synthesis codes to the corresponding semi-empirical model atmospheres of the surface features (Fontenla et al. 1999, 2004, 2009, 2011; Unruh et al. 1999; Shapiro et al. 2010). Semi-empirical models calculate the SSI in a more straightforward way compared to the proxy models. Moreover, semi-empirical models are able to take the CLV of each component into account.

On millennial time scales, Vieira et al. (2011) used cosmogenic isotopes (both ${ }^{14} \mathrm{C}$ and ${ }^{10} \mathrm{Be}$ ) as solar activity indicators, from which the other surface components were deduced, to calculate the TSI over the Holocene. On the other hand, Shapiro et al. (2011) used only ${ }^{10} \mathrm{Be}$ as a proxy of solar activity to linearly calculate the SSI of the "quiet sun component" and, used sunspot number as the "active sun component" after 1610. Therefore, they might have underestimated the overall solar irradiance for the period prior to 1610 owing to the unavailability of the sunspot records.

The present-day semi-empirical models are: SATIRE (Spectral And Total Irradiance REconstruction, Fligge et al. 2000; Solanki et al. 2002b; Krivova et al. 2003; Krivova and Solanki 2005; Krivova et al. 2006, 2007, 2010; Vieira et al. 2011; Ball et al. 2014; Yeo et al. 2014b), including the newest and a more physics-based generation, SATIRE-3D (Yeo et al. 2017b), using state-of-the-art 3-D model atmospheres generated with the 3-D radiation MHD MURaM ${ }^{19}$ simulation (Vögler et al. 2005); (2) SRPM $^{20}$ (Fontenla et al. 1999, 2004, 2006, 2009, 2011); (3) OAR ${ }^{21}$ (Ermolli et al. 2003, 2011, 2013 ; Penza et al.

\footnotetext{
${ }^{19}$ MPS/University of Chicago Radiative MHD.

${ }^{20}$ Solar Radiation Physical Modelling.

${ }^{21}$ Osservatorio Astronomico di Roma.
} 
2003); and (4) $\operatorname{COSI}^{22}$ (Haberreiter et al. 2005, 2008; Shapiro et al. 2010, 2011, 2013).

Among these, the most versatile and advanced semi-empirical model to date is the SATIRE family of models. The model employs different indices of solar magnetic activity, depending on the time scales. Two versions of the SATIRE model, SATIRE-T and SATIRE-M, are employed in this thesis. In the following sections, we will describe the SATIRE models in more detail.

\subsection{SATIRE model}

As most of the currently existing models, SATIRE is based on the assumption that the solar variability on time scales longer than approximately one day is only caused by the solar surface magnetism (e.g., Fligge et al. 2000; Krivova et al. 2003). This assumption is supported by the excellent agreement between the modelled irradiance variations and satellite measurements (Krivova et al. 2003; Wenzler et al. 2006; Yeo et al. 2014b). In the SATIRE model, the solar surface consists of four components: umbra (u), penumbra (p), faculae (f) and network (n), and quiet Sun (qs).

The time-independent intensity spectra, $I(\lambda, \mu)$, of umbra, penumbra and the quiet sun are calculated following Unruh et al. (1999) using the spectral synthesis code (ATLAS9, Kurucz 1993). The model atmosphere of faculae (and network) is adapted from the model P (Fontenla et al. 1999), modified by Unruh et al. (1999). The intensity spectra are functions of wavelength and the heliocentric angle, $\theta($ or $\mu=\cos \theta)$.

Since the ATLAS9 code assumes the local thermodynamic equilibrium (LTE), the reconstructions with the SATIRE model are less accurate in the UV, particularly below $\approx 300 \mathrm{~nm}$. This shortcoming in the SATIRE model is taken care by offsetting the $180-$ $300 \mathrm{~nm}$ segment to match the Whole Heliospheric Interval (WHI) reference solar spectra (Woods et al. 2009), and rescaling the 115 - $180 \mathrm{~nm}$ segment to the SORCE/SOLSTICE measurements (Snow et al. 2005) using empirical factors (details see Yeo et al. 2014b, and Chap. 4).

In addition, the filling factor, $\alpha$, is introduced in the SATIRE model. The physical meaning of filling factor is to describe the fraction of a given area covered by a specific photospheric component. For the models that apply spatially-resolved full-disc magnetograms (i.e., SATIRE-S, -T2 and -3D), the given area is the measurement pixel, and therefore the filling factors are dependent on both time, and $\theta$. For the models that only have spatially-integrated information (i.e., SATIRE-T and -M), the given area is the solar surface area and therefore the filling factors only depend on time.

By examining the distributions and the filling factors of the photospheric components, the solar irradiance at any given time, $F(\lambda, t)$, can be calculated as the sum of the brightness contributed by all the surface components:

$$
F(\lambda, t)=\alpha_{\mathrm{u}}(t) F_{\mathrm{u}}(\lambda)+\alpha_{\mathrm{p}}(t) F_{\mathrm{p}}(\lambda)+\alpha_{\mathrm{f}}(t) F_{\mathrm{f}}(\lambda)+\alpha_{\mathrm{n}}(t) F_{\mathrm{n}}(\lambda)+\alpha_{\mathrm{qs}}(t) F_{\mathrm{qs}}(\lambda) .
$$

In different versions of the SATIRE model, different ways of determining the spatial characteristics of the magnetic components are employed, as described in the following sections.

\footnotetext{
${ }^{22}$ COde for Solar Irradiance reconstruction.
} 


\subsubsection{SATIRE-S}

SATIRE-S (S stands for satellite era) uses spatially-resolved data, such as the longitudinal magnetograms and the full-disc continuum images from the ground-based KPVT (Livingston et al. 1976) and the space-based SoHO/MDI ${ }^{23}$ and $\mathrm{SDO} / \mathrm{HMI}^{24}$, to determine the positions and the fractional disc coverages by the photospheric components (Fligge et al. 2000; Krivova et al. 2003; Wenzler et al. 2004, 2005, 2006; Ball et al. 2012; Yeo et al. 2014b). The magnetic regions are determined from the magnetograms where the magnetogram signal is above the noise floor. The pixels where the continuum intensity is below a certain threshold are identified as sunspots (umbrae and penumbrae), while other pixels are counted as faculae and network, with both being treated as a single component.

However, network elements and faculae are usually smaller than a measurement pixel size and are largely not resolved (Krivova and Solanki 2004). Very often, they cover part of the pixel and share the remaining part of the pixel with the quiet Sun. Therefore, the measured magnetic flux within a pixel is always lower or equal to the intrinsic magnetic flux of the magnetic element. The difference between these two magnetic field strengths implies that the magnetic element only covers a fraction of the measured pixel, which is represented by the filling factor, $\alpha$. The saturation flux, $B_{\text {sat }}$, represents the value when a pixel is fully covered by the same magnetic feature. The filling factors of the nonspot magnetic pixels are considered to be linearly proportional to their pixel-averaged magnetic flux density (i.e., $\alpha=0$, when no magnetic flux and $\alpha=1$, when the $B_{\text {sat }}$ is reached). Since the magnetogram signal corresponding to the faculae model atmosphere is unknown, this saturation level is therefore set as a free parameter in the SATIRE-S model. The value of the saturation level is calculated by comparing the modelled results to the measured TSI/SSI.

The SATIRE-S model is capable of reproducing more than $92 \%$ of the measured solar irradiance for the period $1974-2013$ and even 96\% for the solar cycle 23 (Yeo et al. 2014b). This good agreement supports the fundamental assumption in the SATIRE model that most of the irradiance variations on the considered time scales are due to the evolution of the solar surface magnetic fields (Domingo et al. 2009). Unfortunately, because of the limited availability of the magnetogram measurements, the reconstructions with SATIRE-S can only go back to 1974.

\subsubsection{SATIRE-T}

Unlike the SATIRE-S model that relies on the direct measurements of the surface magnetic fields, the SATIRE-T ( $T$ for telescope era) model uses the sunspot number record (Sect. 2.1) as a proxy of solar activity. This allows the SATIRE-T model to reconstruct TSI/SSI back to the Maunder minimum (Balmaceda et al. 2007; Krivova et al. 2007, 2010; Wu et al. 2018a).

Since the sunspot number only provides disc-integrated information, the AR emergence rate (Chapman et al. 1997; Solanki et al. 2002b; Krivova et al. 2007, 2010) and the ER emergence rate (Harvey and Martin 1973; Harvey 1992, 1993; Schrijver and Harvey

\footnotetext{
${ }^{23}$ Michelson Doppler Imager.

${ }^{24}$ Helioseismic and Magnetic Imager.
} 


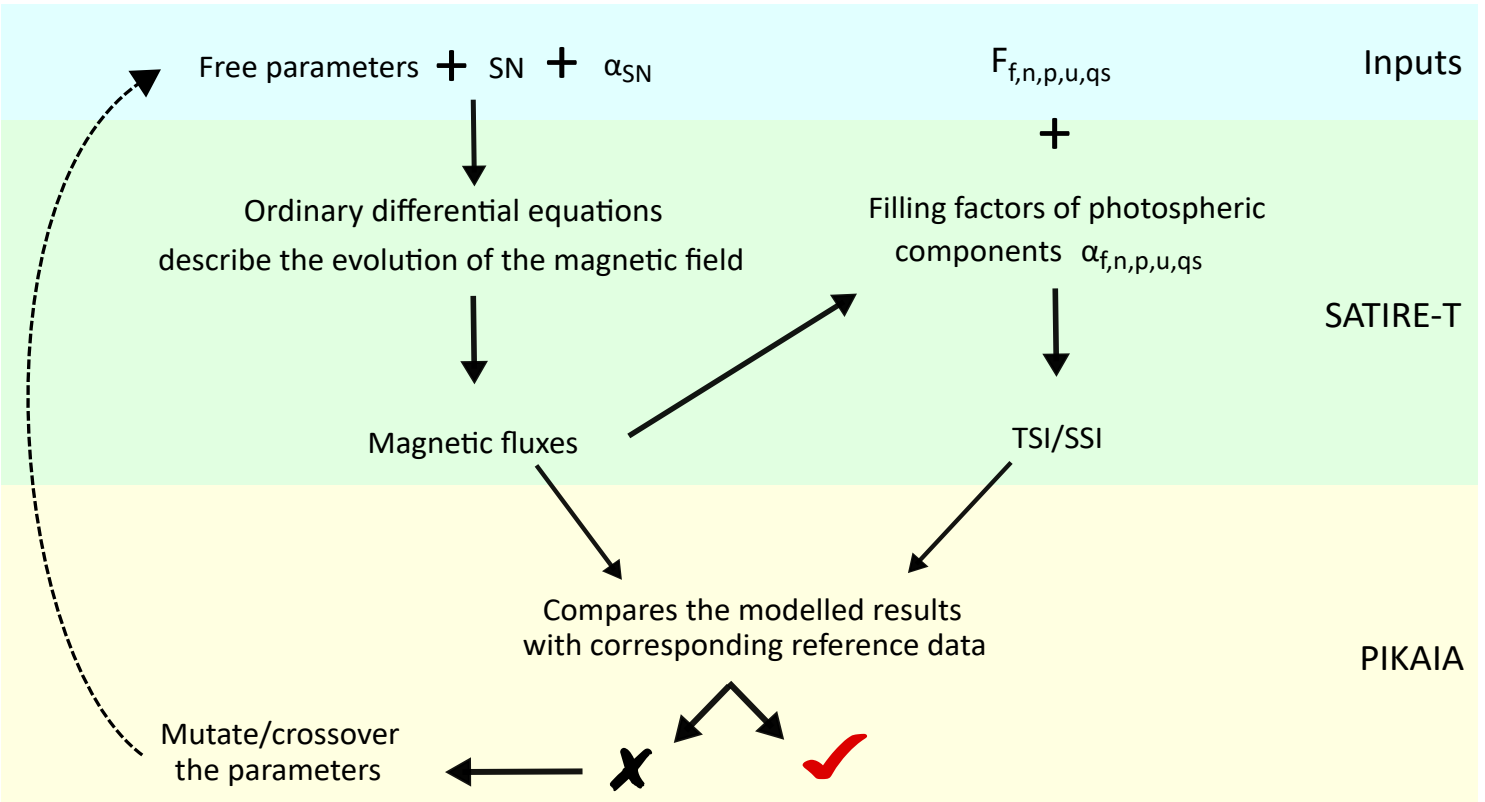

Figure 1.12: Schematic of the SATIRE-T model process. The inputs of the SATIRE-T model are the initial free parameters, sunspot numbers, the sunspot filling factor $(\alpha)$, and the spectral intensities of photospheric components $(F)$. The SATIRE-T model calculates the magnetic fluxes and the solar irradiance, which are later compared with the reference data sets, using the PIKAIA genetic algorithm. The PIKAIA routine mutates the free parameters and feeds back to the SATIRE-T model, until the parameters reach a stable regime.

1994) are derived with the physics-based model (Solanki et al. 2002b). Next, the evolution of ARs, ERs, and open magnetic field (OMF) is calculated using a set of ordinary differential equations (ODEs; Krivova et al. 2010; Vieira and Solanki 2010). The model has a number of free parameters that are fixed from a comparison to various observations (for more details, see Chap. 4).

The free parameters are constrained by comparing the modelled results with the measurements over the overlapping periods using the genetic algorithm, PIKAIA ${ }^{25}$ (Charbonneau 1995). Figure 1.12 is a schematic of the procedure of constraining the parameters. First the sunspot number is fed into the SATIRE-T model as a proxy of solar activity along with the sunspot area, which is extrapolated using sunspot number when the area record was not available. The ODEs return the modelled magnetic fields, which are later used to convert into the filling factors, $\alpha$. By applying the intensity spectra of each component, the SSI at any given time is calculated. Next, the reconstructed magnetic fluxes and TSI/SSI are fed into PIKAIA to compare with the corresponding measured quantities. Then PIKAIA slightly "mutates" the parameters and repeats the process until the parameters reach a stable regime that gives the best agreement of model results with the observations.

\footnotetext{
${ }^{25}$ http://www .hao.ucar.edu/modeling/pikaia/pikaia.php
} 


\subsubsection{SATIRE-T2}

The SATIRE-T2 (SATIRE-T, version 2) model is an advanced version of the SATIRE-T model. It employs a surface flux transport model (SFTM) to simulate the daily magnetograms, and therefore is more accurate on the rotational time scale than the SATIRE-T model (Dasi-Espuig et al. 2014, 2016). The SFTM uses the information on the sunspots (e.g., numbers, positions) to describe the emergence rate of AR magnetic flux. The evolution of the large-scale magnetic field is determined by solving the radial component of the induction equation (Wang et al. 1989; van Ballegooijen et al. 1998; Baumann et al. 2004; Schüssler and Baumann 2006; Cameron et al. 2010; Mackay and Yeates 2012; Jiang et al. 2014).

To simulate the daily maps of the magnetic field on the solar surface with the SFTM, Dasi-Espuig et al. (2014) used the record of the observed sunspot group area and positions from the Royal Greenwich Observatory (RGO) between 1874 - 1976, and from the USAF/NOAA SOON network after 1976. The detailed spatial information on sunspots allows taking CLV of the AR contrast into account. The ERs are treated in the same manner as in the SATIRE-T model. With the simulated magnetograms, the same reconstruction scheme is followed as in the SATIRE-S model (Sect. 1.4.1). Dasi-Espuig et al. (2016) extended the reconstruction back to 1700 using the synthetic butterfly diagram. The daily synthetic butterfly diagram before 1874 was reconstructed by Jiang et al. $(2011 \mathrm{a}, \mathrm{b})$ based on a statistical study of the sunspot-related quantities, such as sunspot number, distributions of latitude and longitude, sunspot area, and tilt angles of sunspot groups.

Although the SATIRE-T2 model has a more realistic description of the AR evolution than the SATIRE-T model, a number of free parameters are still needed. There are in total five free parameters in the model of Dasi-Espuig et al. (2014) and four in that of Dasi-Espuig et al. (2016). As in the SATIRE-T model, these four free parameters are optimized by comparing the modelled results to the measurements using the optimisation routine PIKAIA.

\subsubsection{SATIRE-M}

Directly, i.e. telescopically determined sunspot number, unfortunately, is not available prior to the 17 th century. Therefore, one has to rely on indirect proxies, cosmogenic isotopes (e.g., ${ }^{14} \mathrm{C}$ and ${ }^{10} \mathrm{Be}$ ) in terrestrial archives. The corresponding SATIRE model version using such proxies, is termed SATIRE-M (M stands for millennia, Vieira and Solanki 2010; Vieira et al. 2011; Wu et al. 2018a).

The concentration of the isotope samples retrieved from natural archives can be converted into Sun-related quantities, such as the OMF and solar modulation potential, $\phi$ (Sect. 2.3.1), via a series of physics-based models (Solanki et al. 2004). However, a reconstruction of the sunspot number and TSI/SSI from the OMF is not straightforward, mainly due to a number of non-linear physical processes involved. Since both ARs and ERs contribute to the OMF while decaying, and the emergence rate of ERs is related to that of ARs (Sect. 1.2.2), the OMF can essentially be linked to ARs.

One of the most important differences of the SATIRE-M model from the other SATIRE versions is the temporal resolution of the input data. The original ODEs of the SATIRE-T model is not suitable for the decadally-resolved concentrations of cosmogenic 
isotopes. Therefore, three main changes have been made to account for this: (1) all ODEs are averaged decadally; (2) we assume that ARs, ERs and rapidly evolving open flux evolve steadily on the decadal time scale; and (3) the decadally-averaged sunspot number is taken to be linearly proportional to the maximum sunspot number of the corresponding cycle (Usoskin et al. 2007; Vieira and Solanki 2010). Based on these assumptions, the decadally-averaged sunspot number can be described solely by the decadally-averaged OMFs from two consecutive cycles (see appendix A.1 for the complete derivation):

$$
\langle R\rangle_{j}=a\left\langle\varphi_{\mathrm{open}}\right\rangle_{j}+b\left\langle\varphi_{\mathrm{open}}\right\rangle_{j+1}
$$

where $j$ denotes the $j$-th cycle. The two coefficients, a and $\mathrm{b}$, are obtained from the SATIRE-T model. Equation (1.3) essentially tells us that the OMF of each cycle is formed by the contributions of ARs that emerged during that same and the preceding cycles. This is because part of the emerged magnetic flux is dragged to the poles, building up big unipolar caps of OMF that can survive longer than one cycle length.

The reconstructed sunspot number, $R$, and the OMF, $\varphi_{\text {open, }}$, are then employed in the SATIRE-M model using the same scheme as in the SATIRE-T to reconstruct the solar irradiance at any given time, $\langle F(\lambda, t)\rangle_{j}$, (see appendix A.2 for full derivation):

$$
\langle F(\lambda, t)\rangle_{j}=a_{\mathrm{F}}(\lambda)\left\langle\varphi_{\mathrm{open}}\right\rangle_{j}+b_{\mathrm{F}}(\lambda)\left\langle\varphi_{\mathrm{open}}\right\rangle_{j+1}+F_{\mathrm{q}}(\lambda),
$$

where

$$
(a, b)_{\mathrm{F}}(\lambda)=\sum_{i=\mathrm{u}, \mathrm{p}, \mathrm{f}, \mathrm{n}}(a, b)_{i}\left\langle F_{i}(\lambda)-F_{\mathrm{q}}(\lambda)\right\rangle .
$$

Equation (1.3) and (1.4) suggest that irradiance reconstructions based on a simple linear regression between the OMF and the TSI (e.g., Steinhilber et al. 2009, 2012; Delaygue and Bard 2011; Shapiro et al. 2011) are not justified physically.

\subsection{Thesis outline}

Various studies have suggested a link between changes in solar activity and climate on Earth. Even though growing evidence exists for the largely human origin of the global warming during the last century, accurate quantification of Sun's role in this warming is still pending. The degree of influence of the solar irradiance on the Earth's climate system is, however, very difficult to assess since there are various complicated processes involved. Comprehensive knowledge of the changes in the Earth's climate system requires powerful computation ability, where the accurate energy forcing (including the Sun), detailed atmosphere-ocean coupling, energy transport between reservoirs (e.g., vegetations, volcanic aerosols, mixed GHG and land use; Schmidt et al. 2011) are needed. Therefore, the goal of this thesis is to understand the secular changes in the solar irradiance as well as to quantify and make it applicable to the climate communities.

This thesis is structured as follows. In Chap. 1, we have introduced the physics of the Sun and the models for solar irradiance reconstruction. The input to the SATIRET model, the sunspot number, is still a topic of debate and will be discussed in Sect. 2.1. Further, the SATIRE-M model employs concentrations of the cosmogenic isotopes in natural archives as input. Cosmogenic isotopes are produced by galactic cosmic rays 
(GCRs) whose incoming flux is modulated not only by the Sun but also by the geomagnetic field. To extract the solar signal from the cosmogenic isotope data, one has to properly account for the geomagnetic field. In Sect. 2.2, we will introduce the geomagnetic field and its approximations on millennial time scales. Next, the physics of GCRs and their modulation by the Sun and the Earth are described in Sect. 2.3. Finally, the two most commonly-used cosmogenic isotopes, ${ }^{14} \mathrm{C}$ and ${ }^{10} \mathrm{Be}$, will be introduced in Chap 2.4 .

Due to the different geochemical paths these two cosmogenic isotopes experience, the signals in the data show some discrepancies not only in the temporal behaviour, but also in their absolute levels. In Chap. 3, we will describe our new method to combine one global ${ }^{14} \mathrm{C}$ and six local ${ }^{10} \mathrm{Be}$ series into one consistent multi-isotope composite. The reconstruction of solar activity from this composite is also described in this chapter. In Chap. 4, this composite is taken as input into the SATIRE-M model to reconstruct the solar irradiance on millennial time scales. Since the SATIRE-M model is partially based on the SATIRE-T model, we will first re-visit and update the SATIRE-T model (Sect. 4.2.2). The free parameters constrained from the SATIRE-T are then employed in the SATIRE-M model to reconstruct the SSI series over the Holocene. This is the first full SSI reconstruction based on a series of physics-based models. The reconstructed SSI has, however, a decadal temporal resolution due to the original resolution of the cosmogenic isotope data. This reconstruction would, unfortunately, cause decadal bias in climate models since all other forcing inputs have much higher temporal resolutions (Schmidt et al. 2011). In Chap. 5, we will describe a statistical method, which links the decadallyaveraged sunspot number to the corresponding solar cycle characteristics. With these relationships, quasi 11-year solar cycles can be approximately "postdicted". The summary of this thesis and the outlook will be given in Chap. 6 . 


\section{Background of solar activity proxies}

To better carry out our study, i.e., reconstructing the TSI/SSI on millennial time scales using cosmogenic isotopes, it is crucial to also understand the proxies of solar activity. We will first introduce the input data (sunspot number) used in the SATIRE-T model in Sect. 2.1. Secondly, the geomagnetic field and its approximations are introduced in Sect. 2.2. Thirdly, since our proxy of solar activity used in the SATIRE-M model is produced by high-energy cosmic rays generated outside of the solar system, the galactic cosmic rays are then introduced in Sect. 2.3. The production of the cosmogenic isotopes used in this thesis is described in Sect. 2.4.

\subsection{Sunspot observations}

Sunspots are one of the most visible and easily observable types of features on the solar surface and are important manifestation of solar magnetic activity. Due to the high magnetic concentration (Sect. 1.2), sunspots appear to be darker compared to the ambient solar surface. Sunspots are easily followed across the solar disc due to the solar rotation. Even though the sunspot number $(\mathrm{SN})$ is rather a synthetic than a physical index, it is often seen as an indicator of solar activity and has become a useful and convenient parameter in quantifying solar activity level. In the following, we will give a brief history of sunspot observation and the SN series.

Before any systematic and continuous observation of sunspots, there have been short and sporadic records by naked-eye observations throughout history. The earliest trace of sunspot observations was found on oracle bones in China, which date back to around 1000 BC. Most of the ancient sunspot records can be found in the royal administration records produced by Chinese astrologers, for example in Hán Shü (28 BC), Ho Hán Shü (118 AD) and Sóng Shi (1131 AD). It has also been observed in ancient times that the durations, sizes and shapes of sunspots are irregular. However, the records were made for the Emperors' uses rather than scientific research, the sunspot observation was not continuous and the detailed characteristics of sunspots are therefore missing or vague, which increases the difficulty of quantifying and calibrating the records. Wittmann and Xu (1987), Yau and Stephenson (1988) and Stephenson (1990) have compiled catalogues of the naked-eye observed sunspot records from all around the world covering the period 165 BC to 1918 AD. Figure 2.1 shows the frequency of the sunspot observations in decadal cadence (Yau 1988).

Although the first hand-drawn image of the Sun was made by Gemma Frisius in 1544 (Frisius et al. 1545) with a camera obscura (Fig. 2.2), it was during the Spörer minimum and therefore no sunspot was observed. The first accurate than naked-eye observation of 

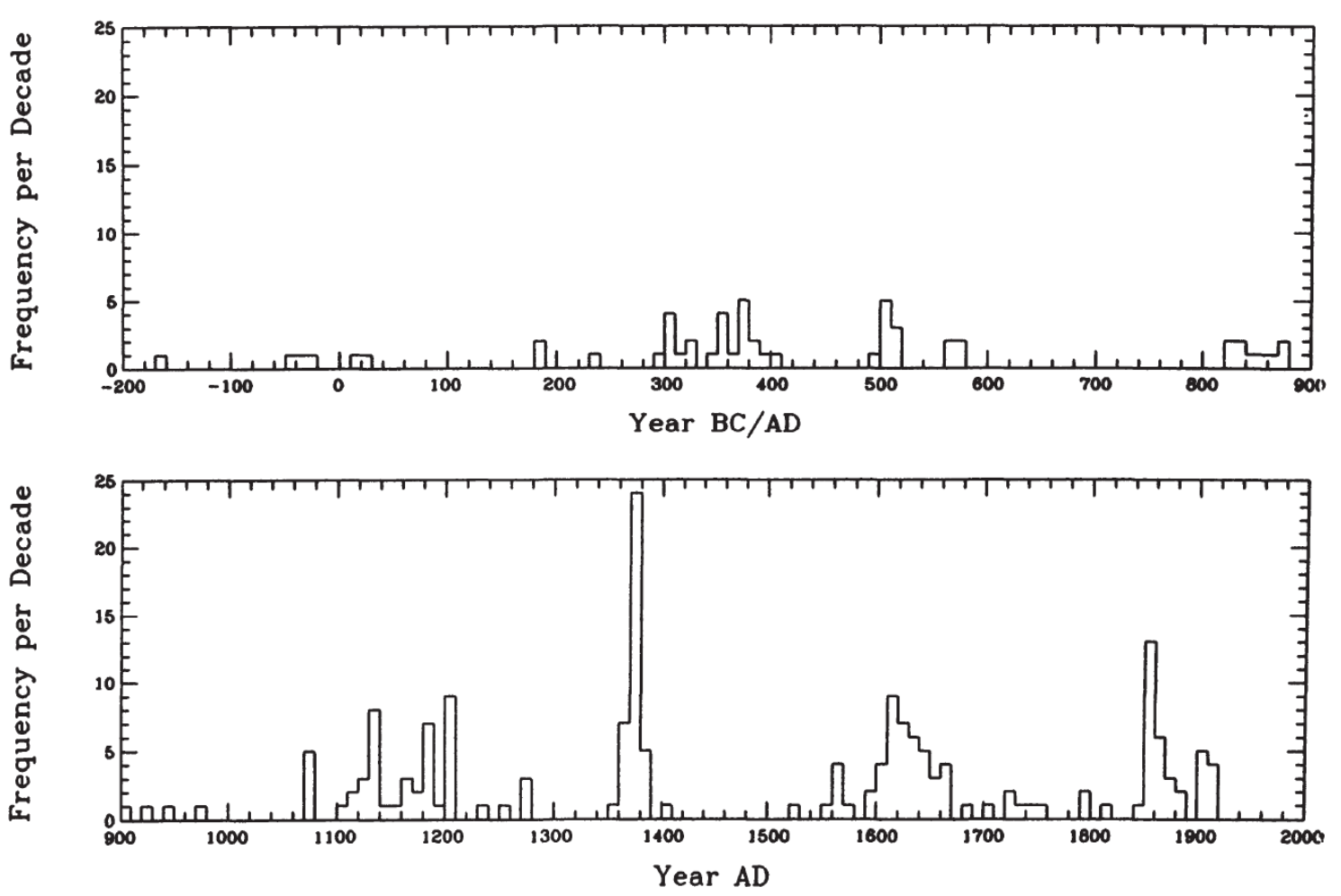

Figure 2.1: Dacadal distribution of naked-eye observed sunspot records from 165 BC to 1918 AD. Taken from Yau (1988).

sunspot was reported by Johannes Kepler with a camera obscura in 1607, who, however, mistook the large sunspot as a Mercury transit (Vaquero 2007). The sunspot observation with camera obscura lasted from the mid 16th to mid 18th century, whereas the majority of the observations were made by telescopes.

Early telescopic sunspot observations were made from multiple observatories and astronomers, who provided in general poor and unsystematic documentation (Vaquero 2007). Two major attempts have been made to compile the available documents. The first one was done by Rudolf Wolf in 1849 (Wolf 1850) and the second by Hoyt and Schatten (1998). Wolf based at Zürich observatory, Switzerland, where he recorded and collected the sunspot numbers from other observers (e.g., Schwabe, Staudacher and Flaugergues). Wolf attempted to produce a systematic and less biased record, which was basically to keep just the number of sunspots observed, corrected for a measure of how well the observer caught the number of sunspots on the disc. Wolf's sunspot number is known as the Zürich sunspot number, which has been maintained and updated continuously by successors (Waldmeier 1961; McKinnon and Waldmeier 1987; Vanlommel et al. 2004). This series is also known as the international sunspot number, ISN. Due to various technical issues, such as weather, observation equipments or observer-related subjective criteria, Wolf defined the sunspot number, $R_{\mathrm{z}}$, as

$$
R_{\mathrm{z}}=k(10 G+S),
$$

where $k$ is a correction (scaling) factor for the normalization between multiple independent observers, $G$ is the number of observed sunspot groups and $S$ is the individual observed sunspots in all sunspot groups. Because of the insufficient observations in the early 


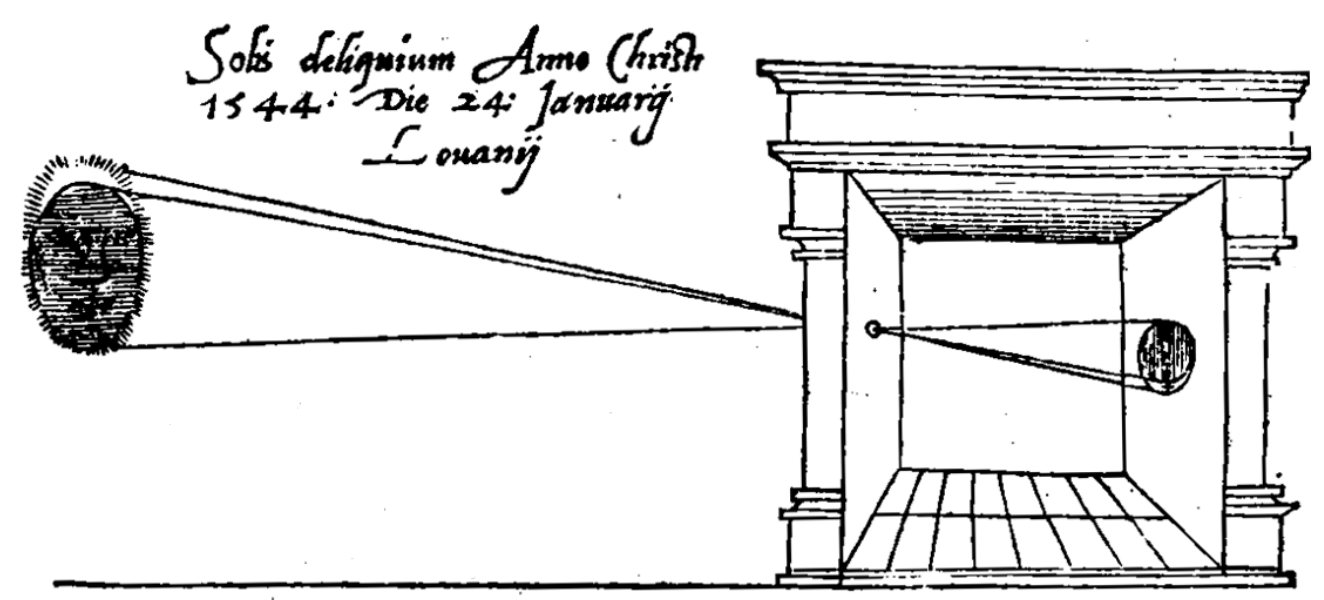

Figure 2.2: Drawing of a camera obscura for astronomical use. Adapted from Frisius et al. (1545).

years, the ISN series has three types of temporal resolutions: 1700 - 1749 (annual), 1750 - 1817 (monthly) and 1818 - present (daily). Wolf deliberately ignored any sunspot that was small and short-lived and the ones without penumbra (usually referred to pores). He also counted sunspots that share the same penumbra, as one sunspot (Clette et al. 2014). Wolf's successor Alfred Wolfer, continued to record sunspots in Zürich but changed these criteria. With his 17-year $(1877$ - 1893) parallel observation with Wolf, Wolfer derived a stable average ratio between Wolf's counts and his higher counts. A factor of 0.6 was obtained through the comparison and has to be multiplied to bring Wolfer's counts to Wolf's level. Since 1981, the ISN series was synthesized by the Royal Observatory of Belgium, Brussels. This series has recently been updated from the original version 1.0 to version 2.0 with corrections for some inhomogeneities (Clette et al. 2014). In contrast to the ISN (v.1), which is calibrated to Wolf's number, the ISN (v.2) takes Wolfer's sunspot number as reference.

The second attempt was done by Hoyt and Schatten (1998), who made a new construction of the group sunspot number (GSN) series (HS98 hereafter). This series recorded only the number of sunspot groups and ignored individual sunspots. The GSN series, $R_{\mathrm{g}}$, is extended back to 1610 and is defined as

$$
R_{\mathrm{g}}=\frac{12.08}{N} \sum_{i} k_{i} G_{i}
$$

where $G_{i}$ is the GSN reported by $i$-th observer with their individual correction factor $k_{i}$, and $N$ is the number of observers that observed at the given day. The constant 12.08 is a normalization factor introduced to match the average of $R_{\mathrm{g}}$ to that of $R_{\mathrm{z}}$ over the period 1874 to 1976 . The HS98 series has been considered as a robust estimate of solar activity due to the omission of individual sunspots, which are an important source of uncertainty, as small individual sunspots are much more likely to be missed by a given observer than a whole sunspot group. The original HS98 series was tabulated daily from 1610 to $1995^{1}$.

\footnotetext{
${ }^{1}$ https://www.ngdc.noaa.gov/stp/space-weather/solar-data/solar-indices/ sunspot-numbers/group/daily-input-data/
} 
There is no doubt that Hoyt and Schatten (1998) performed an important piece of work and their series has been recommended for solar activity analysis. Yet, the HS98 series contains errors and mistakes that were reported recently (e.g., Vaquero 2007; Vaquero et al. 2012; Vaquero and Trigo 2014; Clette et al. 2014; Usoskin et al. 2016c). For instance, the confusing use of the Gregorian and the Julian calendars, and misplacement of observation dates, causing the wrong dates of the sunspots in the early period. Furthermore, methodological inconsistency and partial information from eclipses might lead to an over/underestimation of the SN (Vaquero 2007).

Several attempts have been made to revisit the GSN series using different calibration methods. Lockwood et al. (2014c) applied correction by including a $20 \%$ decrease in the GSN before 1849 (Leussu et al. 2013) and an 11.6\% decrease in the GSN after 1940 (Lockwood et al. 2014b) to account for the Wolf discontinuity ${ }^{2}$ and the Waldmeier discontinuity $^{3}$, respectively.

Svalgaard and Schatten (2016) reconstructed a series (SV16, hereafter) by averaging the values from four methods: daisy-chaining, backbone, high-low ${ }^{4}$ and brightest star ${ }^{5}$. For the period 1749 - 2015, Wolfer's series is taken as a reference observer to calculate the daisy-chaining $k$-factors of other observers. To reduce the steps in the daisy-chain method, the backbone method is introduced by choosing five key observers ${ }^{6}$ (called backbones) and calibrating the other series to the overlapping periods of the backbones. For the entire period over 1610 - 2015, high-low and brightest star methods are applied by keeping the highest counts among the observers. The resulting series also extends back to 1610 (same as HS98) and indicates a higher level of solar activity from Maunder minimum to mid 19 th century than suggested by HS98.

Cliver and Ling (2016) reported a potential unsteady quality in the HS98 series before 1915, which is, however, not found by Lockwood et al. (2016) and Willamo et al. (2017). Therefore, Cliver and Ling (2016) might have over-corrected the data, which resulted in the highest level among all available GSN series. Furthermore, Usoskin et al. (2016b) showed that the linear regression methods of re-calibrating the sunspot number series from individual observers performed by all other studies are not realistic. Usoskin et al. (2016c) presented the first GSN series using a non-linear calibration method. This method is based on statistics of the active day fraction (ADF), which is defined as the ratio of days with observed sunspots to the number of observation days by the same observer in a month. This non-linear method is free from any inter-calibration between observers and the error propagation resulting therefrom. This series results in a moderate solar activity level between the HS98 and the SV16 series between the 18th and 19th century. Recently, a constructed GSN series extending back to 1739 was published by Chatzistergos et al. (2017), who also performed a non-linear non-parametric calibration based on probability distribution functions (PDF) of several backbone observers. The error of this series is estimated with Monte Carlo simulations.

\footnotetext{
${ }^{2}$ Wolf discontinuity is a sudden decrease in the ISN compared to GSN series during 1848 - 1864 (Leussu et al. 2013).

${ }^{3}$ Waldmeier discontinuity (jump) refers to a sudden jump in the ISN series around 1945 (Svalgaard 2011).

${ }^{4}$ The high-low method is to choose the observers who reported a larger GSN than other observer.

${ }^{5}$ The brightest star method is to take the highest daily GSN per year.

${ }^{6}$ The five backbones are Staudacher $(1749$ - 1799), Schwabe (1828-1867), Wolfer $(1878-1928)$, Koyama (1947 - 1980), and Locarno (1958 - 2015).
} 


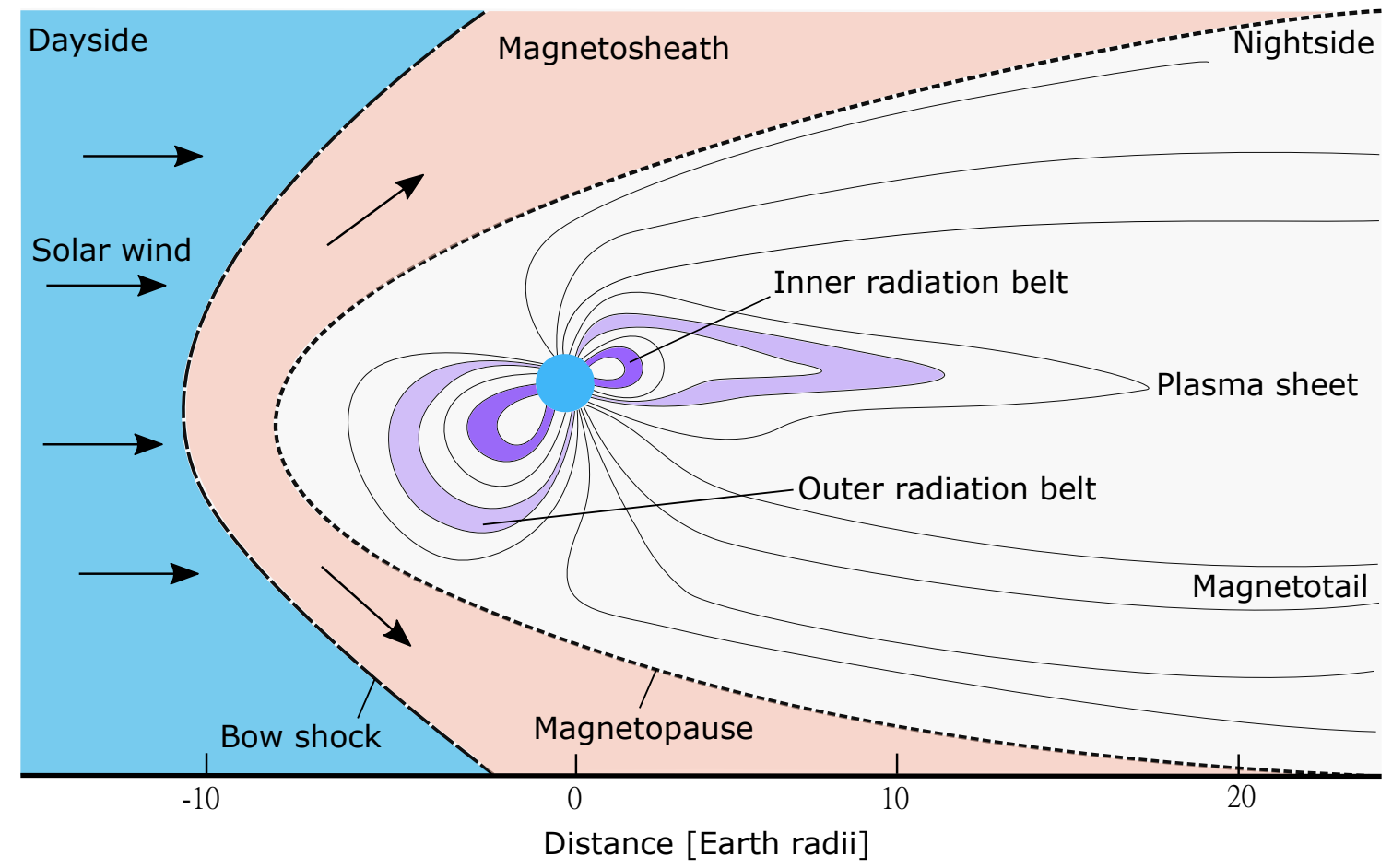

Figure 2.3: Illustration of the Earth's magnetosphere.

All the re-calibrated sunspot number series described above are reconstructed from existing data sets and it is not trivial to determine which series describes the reality best. With the implementation of the sunspot numbers in reconstructing the solar irradiance (Sect. 1.4.2), the choice of input series with an increasing long-term trend (Hoyt and Schatten 1998; Usoskin et al. 2016c) or a rather constant long-term trend (Cliver and Ling 2016; Svalgaard and Schatten 2016) leads to different TSI/SSI reconstructions (Kopp et al. 2016; Lockwood et al. 2016).

However, since the major differences among all the proposed revised SN series lie between the 18th -19 th centuries while the overall solar activity of the 17th and the 20th centuries are in agreement, the choice of the sunspot number series does not affect the secular trend in solar activity from the Maunder minimum to the modern maximum (Kopp 2016; Wu et al. 2018a).

\subsection{Geomagnetic field}

The Chinese discovered, more than 1000 years ago, that a needle always aligns to the direction of north-south when floating on water. Since lodestone is a naturally magnetized piece of the mineral magnetite, this discovery eventually led to the invention of the modern compass. Soon after, the compass was widely used for navigation and has increased the speed of civilisation all around the world. In 1600, William Gilbert first described the Earth as a giant magnet. Two centuries later, a mathematical model was developed by Carl Friedrich Gauß, describing the source of the majority of the measured magnetic field origins from the interior of the Earth. The Earth's magnetic field is briefly introduced in the following. 
The Earth's magnetic field, also called the geomagnetic field, was later found to be generated in the Earth's interior through a self-sustaining dynamo process in the Earth's core, which consists of electrical currents driven by rotation and convection of the viscous material (e.g., Glatzmaier and Roberts 1995; Glatzmaier et al. 2004; Olson et al. 1999; Buffett 2000). The geomagnetic field extends outwards from the interior of the Earth into interplanetary space. This field surrounds and protects the Earth from energetic charged particles. The general shape of the geomagnetic field can be approximated by a dipole. However, due to the solar wind, the geomagnetic field is compressed at the dayside and extended at the nightside. Therefore, the shape of the geomagnetic field appears like a comet-tail, as illustrated in Fig. 2.3. The dayside magnetopause is at about 10 Earth's radii $\left(R_{\mathrm{E}}\right)$ and the tail of the magnetosphere extends to at least $100 R_{\mathrm{E}}$ (Axford et al. 1965; Dessler and Juday 1965; Ness 1969).

The geomagnetic field can be described with three orthogonal components at any given point on the Earth's surface. One of the common systems is a Cartesian coordinate system, $(X, Y, Z)$, where $X$ points geographically northwards, $Y$ points eastwards and $Z$ points downwards (into the Earth). The magnetic field vector at any point is described as $\mathbf{B}$ with magnetic field intensity $B . \mathbf{H}$ is the projection of $\mathbf{B}$ on the horizontal plane with an intensity $H$, defined as $\sqrt{X^{2}+Y^{2}}$, as shown in Fig. 2.4. An alternative common way to specify any given point on Earth is by its total magnetic intensity $B$ and two angular quantities: declination $(D)$ and inclination $(I)$. The declination is the angle between the horizontal direction and the geographic north, $D=\tan ^{-1}\left(\frac{Y}{X}\right)$. The inclination is defined as the angle between the total field vector $\mathbf{B}$ and the horizontal plane, $I=\tan ^{-1}\left(\frac{Z}{H}\right)$.

Since the magnetic field $\mathbf{B}$ is a conservative force (i.e., $\nabla \times \mathbf{B}=0$ ), the field can be expressed as a gradient of a scalar potential $V$ as

$$
\mathbf{B}=-\nabla V .
$$

Using the solenoidal characteristic of the magnetic field $(\nabla \cdot \mathbf{B}=0$, i.e., magnetic monopoles do not exist), we can derive Eq. (2.3) as a Laplace's equation:

$$
\nabla^{2} V=0 .
$$

The scalar potential of the geomagnetic field $V$ is a function of radius $r$, co-latitude $\theta$, and longitude $\phi$ (Chapman and Bartels 1940). By assuming the Earth is a sphere (and ignoring the external source term of the magnetic field which is small and transient), the solution of Laplace's equation (Eq. 2.4) is usually described by spherical harmonic analysis (SHA):

$$
V(r, \theta, \phi)=R_{\mathrm{E}} \sum_{l=1}^{\infty} \sum_{m=0}^{l}\left(\frac{R_{\mathrm{E}}}{r}\right)^{l+1}\left(g_{l}^{m} \cos (m \phi)+h_{l}^{m} \sin (m \phi)\right) P_{l}^{m}(\cos \theta),
$$

where $P_{l}^{m}$ are the Schmidt quasi-normalized Legendre functions of degree $l$ and order $m$ (e.g., Backus et al. 1996). The $g_{l}^{m}$ and $h_{l}^{m}$ are known as Gaussian coefficients, which are determined by fitting observational data to Eq. (2.5). The geomagnetic field is composed of a main magnetic dipole component and other small non-dipole components, described by the Gaussian coefficients. The magnitude of the Gaussian coefficients decreases with increasing order, i.e., the higher order the coefficients, the less significant contribution to the whole magnetic field. 


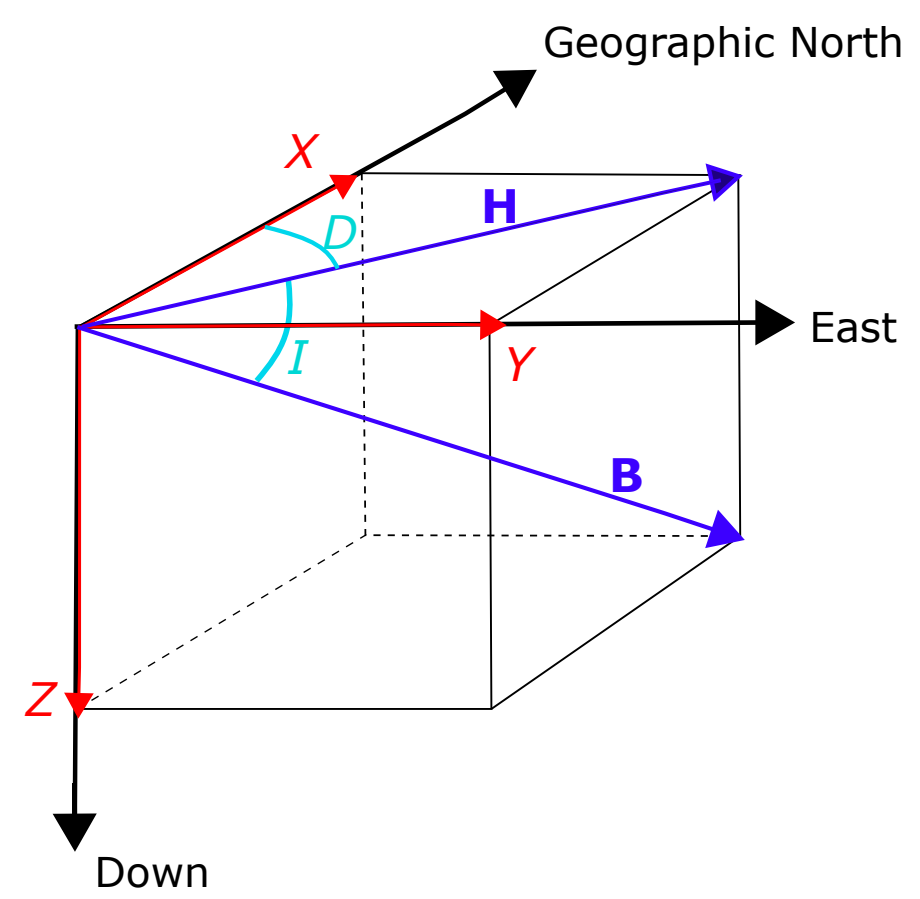

Figure 2.4: Sketch of coordinates used to describe magnetic fields. $\mathbf{B}$ is the vector representing the magnetic field of the earth with magnitude $B . \mathbf{H}$ is the projection of the field, $\mathbf{B}$, onto the horizontal plane. $X, Y$ and $Z$ represent the northward, eastward, and downward directions, respectively. Declination, $D$, is the angel between $\mathbf{H}$ and the geographic north. $I$, inclination, is the angle between $\mathbf{B}$ and the horizontal plane.

The geomagnetic field is not a static field. Instead, it varies significantly with time throughout the Earth's history. There have been discoveries of the changes in the strength and direction of the geomagnetic field. One of the extreme changes in the geomagnetic field history is the geomagnetic reversal. Evidence for these events can be found near the mid-ocean ridges, where the rocks were magnetized when the lava temperature cools down and pushed aside by new volcanic activities, and the magnetization is preserved until today. The reversal has a pattern of occuring on average every few hundred thousand years (Gubbins 1994; Merrill and McFadden 1999; Rüdiger and Hollerbach 2004) with reversal durations lasting from a few thousand up to 28000 years (Clement 2004). Yet the mechanism of reversal is still a mystery. From the remnant magnetization measurements in ocean sediments and lava flows, geologists have established a last full reversal event, the Brunhes-Matuyama event. The Brunhes-Matuyama event occurred at about $778000 \pm$ 2000 years ago with a duration of about 12000 years (Singer and Pringle 1996; Gradstein et al. 2005). From the last full reversal till now, there was only one short event confirmed. The Laschamp excursion, which took place about $41000 \pm 2000$ years ago, was a short and not complete reversal. The Laschamp excursion can be seen in many geological archives as well as from the long ${ }^{10} \mathrm{Be}$ flux records in ice cores, such as GRIP (Greenland Ice Core Project) and GISP (Greenland Ice Sheet Project), which show an increase in flux by a factor of 2 during this period (see Fig. 1 in Muscheler et al. 2005).

On shorter time scales, the location of the geomagnetic pole has also been discovered to be varying. This phenomenon is known as polar wander. A recent study shows that 
the geomagnetic north pole moves quickly (tens of kilometres per year) towards the north (McElhinny and McFadden 2000). The magnetic north pole, which was in Siberia at about $1000 \mathrm{AD}$, had moved to its presents position in northern Canada (see Fig. 5.8.1-1 in Beer et al. 2012). The geomagnetic poles are offset from the rotation axis by $\approx 11^{\circ}$ and the closest distance between the centre of mass of the Earth and the magnetic axis is $\approx 450 \mathrm{~km}$.

To estimate the characteristics of the geomagnetic field in the past, one requires the information collected from either archaeology or geology. These two corresponding methods are termed "archaeomagnetic studies" and "paleomagnetic studies", respectively (McElhinny and McFadden 2000). Both methods are built based on a common fact: under certain conditions, the magnetic elements in the rocks align with the local geomagnetic field and the degree of magnetization is proportional to the geomagnetic field strength at that point of time. After some time, the magnetization is locked in the rocks and remains even if the local geomagnetic field changes. By measuring the strength and the direction of the magnetization in rocks, the properties of the corresponding local magnetic field at any given point of time can be determined. Based on this concept, as long as one determines the time when the magnetization of the rocks was created and locked, the varying geomagnetic field in the past can be reconstructed.

The first method of determining the time when the rock was magnetized is through archaeological sampling. When early humans used fire or made goods out of clay by firing it, the magnetization of the rocks was reset during this process. Due to human activity throughout history, archaeomagnetic studies provide a large amount of data and information about the past 10000 years. The second method of determining the magnetization time is by analysing rocks formed via natural geological processes. The most common example is the magnetization through volcanic activities. The rocks lose their original permanent magnetic properties when its temperature reaches the Curie temperature $\left(T_{\mathrm{C}}\right)$ of the corresponding material and then is reset according to the local geomagnetic field when the rocks cool down. In addition, the magnetic moments in the sediments align with the local geomagnetic field and are locked when the sediments reach a certain degree of compaction. This method allows for investigation back to $\approx 1000000$ years ago. It is important to note that both methods have uncertainties either caused by human activity (e.g., relocation of the goods and rocks) or by natural events (e.g., earthquake and subsidence). Furthermore, certain chemical reactions caused by lightning can change the characteristics of the magnetization in the rocks.

However, in most paleomagnetic studies, the data sampling is not sufficient to reconstruct the complex magnetic structure. Therefore, the evolution of Earth's magnetic field is often simply described by an axial dipole. Virtual dipole moment (VDM, Korte and Constable 2005, 2006) and Virtual axial dipole moment (VADM, McElhinny and Senanayake 1982; Yang et al. 2000; Usoskin et al. 2016a) are often used to approximate the true dipole moment (DM) and to describe the magnetic field from a limited amount of available archaeo- and palaeomagnetic data (Constable 2007). The VDM is based on the assumption that the field originates only from a geocentric dipole (i.e., the centre of the geomagnetic dipole is located the centre of the Earth and the axis aligns the true magnetic axis) while the VADM assumes the field originates from a geocentric axial dipole (i.e., the centre of the geomagnetic dipole is located at the centre of the Earth and the axis aligns with the rotational axis). However, such simple assumptions about the magnetic structure 
might lead to significant uncertainties and cause systematic errors (Korte and Constable 2005). In addition, the VDM and VADM are limited by the temporal resolution since they are typically averaged over a long period of time (several centuries to millennia), to reduce the contribution of higher degree complex fields. Different from SHA, the higher degree of the contributions cannot be taken into account in either VDM or VADM. Therefore, the dipole moments $(P)$ of VDM and VADM are determined from the measured paleointensity $F$ :

$$
P=\frac{4 \pi}{\mu_{0}} r^{3} \frac{F}{\sqrt{1+3 \cos ^{2} \theta}} .
$$

The dipole moment $P$ is a VDM if the $\theta$ is geomagnetic colatitude, and is VADM if the $\theta$ is geographic colatitude. The geomagnetic colatitude can be determined if the inclination $I$ is known $(\tan I=2 \cot \theta)$, which is, however, often not available. Therefore, the VDM is reliable on time scale up to about 3000 years due to a good global coverage of the samplings, while the VADM is most commonly used on the time scales over the Holocene. The dipole moment averaged over the past $0.3 \mathrm{Myr}$ is about $8 \times 10^{22} \mathrm{Am}^{2}$ (Selkin and Tauxe 2000).

\subsection{Cosmic rays}

Cosmic rays (or cosmic radiation) are produced in high-energy events in the universe. Many origins of cosmic rays have been proposed, such as supernovae (Baade and Zwicky 1934), supernova remnants (Sekido et al. 1951), magnetic variable stars (Babcock 1948), active galactic nuclei (Biermann and Strittmatter 1987; Protheroe and Szabo 1992), quasars (Torres et al. 2002), gamma-ray bursts (Millikan and Bowen 1931) and, at the lowenergy end from the Sun. Cosmic rays consist of $87 \%-90 \%$ of protons (hydrogen nuclei), $9 \%-12 \%$ of alpha particles and the rest less $<1 \%$ nuclei of heavier elements (Masarik and Reedy 1995; Biswas 2000). Cosmic rays originating outside the solar system are called galactic cosmic rays (GCRs). The energy of GCRs is mostly stronger than a few GeV, and the differential energy spectrum of the GCRs follows a power-law function.

Cosmic rays can also be produced from the Sun when the Sun emits high energy particles during extreme high-energy events, such as solar flares and CMEs. In this case, they are called solar cosmic rays (SCRs), but also known as solar energetic particle (SEP) events. SCRs with energy of a few tens of $\mathrm{MeV}$ are frequently detected by satellite-borne measurements whereas SCRs with higher energy (a few hundreds of MeV) are detected on the Earth's surface, although rarely (Reames 1999). Since SCRs have lower energies than GCRs, SCRs contribute less than $1 \%$ to the production of cosmogenic isotopes in the terrestrial environment (Sect. 2.4). In this thesis, we focus on the type of cosmic rays that originate outside of the solar system (viz. GCRs). Due to the long duration of the passage of the solar system through the spiral arms of the galaxy (Shaviv 2002), the influx of GCRs is considered roughly constant in the heliosphere on time scales of millions of years (e.g., Beer 2000a).

However, one should note that the term "ray" (or "radiation") is not accurate term but rather as a historical accident due to its discovery. The first accidental discovery of cosmic rays was by Austrian physicist Viktor Hess in 1912 with a balloon flight, which reached up to $\approx 5 \mathrm{~km}$ (Hess 1912; Beer et al. 2012; Bertolotti 2013). From his instru- 
ments onboard of that balloon, he detected the discharge rate decreases with height up to $\approx 1.5 \mathrm{~km}$ and increases again dramatically to $\approx 5 \mathrm{~km}$, which is twice of the discharge rate at the Earth's surface. This phenomenon was explained by an extra penetrating radiation entering the Earth's atmosphere, which he called "ultra-high energy radiation" (Ultrahochenergiestrahlung).

Since the 1930s, there have been two means of measuring the cosmic rays: (1) continuous ground-based instruments, such as the ionization chambers (Compton et al. 1934; Lange and Forbush 1948; McCracken and Beer 2007), the Geiger-Müller counter (e.g., Geiger and Müller 1928) and various neutron monitors around the globe (e.g., Simpson 1953; Carmichael et al. 1968; Simpson 2000; Dorman 2004; Moraal and Stoker 2010; Mavromichalaki et al. 2016; Usoskin et al. 2017); (2) space-based measurements, such as balloon-borne ionization chambers (Neher et al. 1953; Neher 1971; McCracken and Beer 2007), a short test flight of the spectrometer AMS-017 in 1998 (Alcaraz et al. 2000), satellites (e.g., McDonald 1998), the precise long-running spectrometer PAMELA ${ }^{8}$ (Adriani et al. 2013), and AMS-02 (Aguilar et al. 2015) in the last decade.

A long record of GCRs can be constructed from the continuous neutron monitor records since 1951, and can be extended back to 1933 using ionization chambers and flown balloon measurements. However, the resolution and accuracy also decrease when going back in time.

\subsubsection{Heliospheric modulation}

When GCRs reach $\approx 150$ AU from the Sun, they enter the heliosphere. It is the region of space in which the solar wind dominates energetically over the interstellar medium. There, the extended solar open magnetic flux is carried outwards from the Sun by the solar wind. The strength and the direction of the heliospheric magnetic fields vary with time in accordance with the solar magnetic activity. As a result, GCRs are influenced by the ambient magnetic fields, or "modulated" by the Sun (Parker 1965; Toptygin 1985). The modulation involves four main processes: (1) curvature and gradient drifts of particles caused by the magnetic field; (2) diffusion of particles caused by the inhomogeneous magnetic field; (3) convection of particles by the solar wind; and (4) adiabatic energy loss due to the expansion of the solar wind. Typically, the GCRs intensity below $20 \mathrm{GeV}$ are greatly reduced when passing through the heliospheric magnetic field (Beer et al. 2006, 2012).

Forbush (1938) found that cosmic rays are influenced by the solar activity after reporting a solar flare followed by a sudden decrease in the cosmic ray intensity, a so-called Forbush decrease. Following this, the modulation of GCRs, most prominently, the 11year solar cycle, has been systematically observed by numerous instruments since the 1950s. As shown in Fig. 2.5, the GCR flux density (quantified as count rate measured by a neutron monitor, panel b) is anti-correlated with solar activity (sunspot number, panel a). The cosmic ray counts are normalized to the mean in May 1965. Due to the configuration of the heliospheric current sheet and the unusually weak heliospheric magnetic field, the GCR flux increased to the highest recorded level in 2009 (McDonald et al. 2010).

\footnotetext{
${ }^{7}$ Alpha Magnetic Spectrometer

${ }^{8}$ Payload for Antimatter Matter Exploration and Light-nuclei Astrophysics.
} 

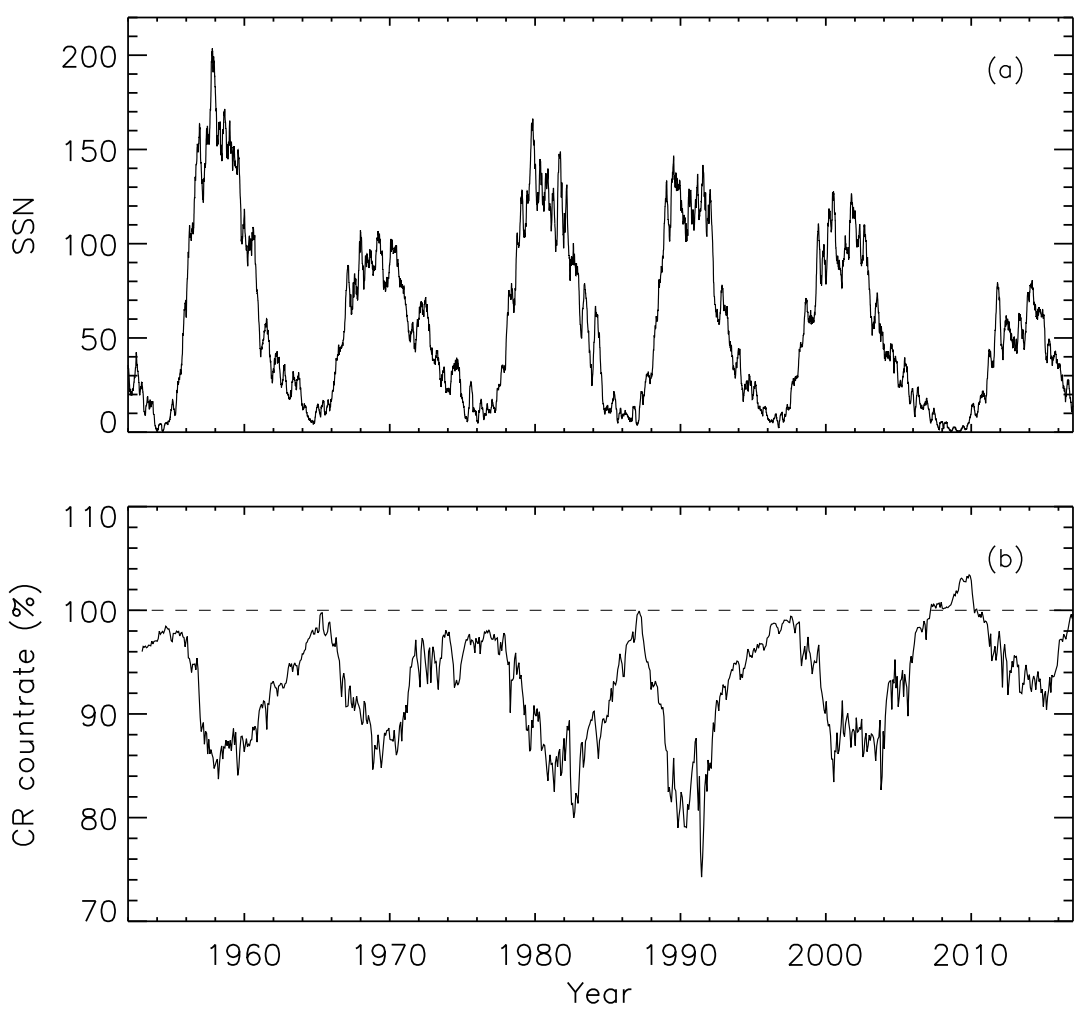

Figure 2.5: (a) Variation of solar cycle (sunspot number ${ }^{9}$ ) and (b) cosmic ray flux measured by neutron monitors since 1951. Data from the Climax $\mathrm{NM}^{10}$ are used after 1951 and from Oulu NM ${ }^{11}$ after 1964. The counts are normalized to that of May 1965 (dashed line).

Forbush (1954) and Dorman and Dorman (1967) have found a general delay in the GCR cycles with respect to the solar cycles. This time-lag has also been found to vary from one cycle to another (Nagashima and Morishita 1979; Marmatsouri et al. 1995), more specifically, from odd-numbered to even-numbered cycles. In cycles 20 and 22, the GCR cycles were delayed by about 2 months whereas a $10-14$ month time-lag has been observed in cycles 19,21 and 23. This behaviour can be explained by the transport and drift process for GCRs in the heliosphere and the time-dependent current sheet tilt angles (Ferreira and Potgieter 2004).

To better understand the transport and the modulation of GCRs in the heliosphere, theoretical and experimental studies have advanced greatly in the last 75 years (for details, see review by Potgieter 1998). Broadly, the description of the modulation process can be categorized into two groups.

The first one is the "cosmic ray propagation function", which describes the combination of the four transportation processes (Parker 1963):

$$
\frac{\partial U}{\partial t}=\nabla \kappa \nabla U-\nabla(V U)-v_{\mathrm{D}} \nabla U+\frac{1}{3} \nabla \cdot \nabla \frac{\partial}{\partial T}(\alpha T U),
$$

\footnotetext{
${ }^{9}$ http://www.sidc.be/silso/

${ }^{10}$ http://cro.izmiran. ru/clmx/main.htm

${ }^{11}$ http://cosmicrays.oulu.fi/
} 
where $U$ is the cosmic ray number density per unit kinetic energy interval, $E$, at time, $t$. $V$ is the solar wind speed, $v_{\mathrm{D}}$ is the effective drift speed in the mean magnetic field, $\kappa$ is the diffusion tensor, and $\alpha=\left(E+2 E_{0}\right) /\left(E+E_{0}\right)$ is a function of the kinetic energy, where $E_{0}$ is the particle rest mass. The four terms on the right-hand-side in Eq. (2.7) describe the diffusion term, the convection term, the drift term, and the deceleration term, respectively. Although the cosmic ray propagation function is accurate, it is, unfortunately, difficult to use and cannot be solved analytically.

The second description of the modulation process is called the "modulation function", which is based on a quasi-empirical approximation, and can be solved analytically (Gleeson and Axford 1968; Caballero-Lopez and Moraal 2004):

$$
J_{i}(E, \phi)=J_{\mathrm{LIS}, i}\left(E+\Phi_{i}\right) \frac{E\left(E+2 E_{0}\right)}{\left(E+\Phi_{i}\right)\left(E+\Phi_{i}+2 E_{0}\right)},
$$

where $J_{i}$ is the differential cosmic ray flux of a cosmic nucleus $i, J_{\text {LIS }}$ is the differential local interstellar spectrum (LIS, cosmic ray flux outside the heliosphere), $\Phi_{i}$ is the modulation function of a cosmic nucleus $i, E_{0}$ is the particle rest mass which has a value of 938 $\mathrm{MeV}$, and $\phi$ is the modulation potential.

Due to the unknown value of $J_{\mathrm{LIS}}$, which is a measured value outside of the heliosphere, it has been approximated by Garcia-Munoz et al. (1975), Burger et al. (2000) and Webber and Higbie $(2003,2009)$. Their values are in agreement for the GCR energy above $20 \mathrm{GeV}$ while the uncertainties could reach up to a factor of 1.5 for GCR energy of $1 \mathrm{GeV}$. These uncertainties result in the modulation theory to be model-dependent (Usoskin et al. 2005; Herbst et al. 2010).

In Eq. (2.8), the differential GCR intensity is described by the modulation potential, $\phi$, which is a time-dependent parameter. It describes the rigidity loss of GCR particles in the heliosphere (i.e., the loss of particles' momentum per unit charge). The notations of modulation function, $\Phi$, and modulation potential, $\phi$, have often been misused and created confusion. To clarify, these two quantities are related by the charge number, $\mathrm{Z}$, and the electron charge, as follows:

$$
\Phi=\mathrm{Z} e \phi
$$

Therefore, the modulation function, $\Phi$, has a unit of $\mathrm{MeV}$ while the modulation potential, $\phi$, has a unit of MV. For the majority of cosmic ray particles (protons), the two units give the same value (i.e., $\Phi_{\mathrm{p}}=e \phi_{\mathrm{p}}$ ). For alpha particles and heavier elements, the relationships are $\Phi_{\alpha}=2 \Phi_{\mathrm{p}}$ and $\Phi_{\mathrm{h}}=\mathrm{Z} \Phi_{\mathrm{p}}$, respectively.

\subsubsection{Geomagnetic modulation (shielding effect)}

After penetrating the heliospheric magnetic field, part of the GCRs travel into the inner solar system to the near-Earth environment. The interplanetary magnetic field near the Earth has an average magnetic strength of $6 \mathrm{nT}$, which is by a factor of about $10^{4}$ weaker than the strength of the geomagnetic field $(\approx 25-65 \mu \mathrm{T})$. Therefore, GCRs that produce cosmogenic isotopes in the Earth's atmosphere are modulated not only by the heliospheric magnetic field (Sect. 2.3.1) but also deflected by the geomagnetic field, as we will introduce in the following. 
The geomagnetic effect on the charged particles in GCRs is called the shielding effect (Vonmoos et al. 2006). This effect is usually described with a cut-off rigidity, $P_{\mathrm{c}}$, which is the minimum rigidity of a vertically-entering cosmic ray needed to reach the terrestrial surface at a given place and time (Cooke et al. 1991). The rigidity is defined as the particle momentum per unit charge and is independent of nuclide species. Therefore, rigidity is a more convenient unit than particle energy. The cut-off rigidity, $P_{\mathrm{c}}$, depends strongly on the geomagnetic latitude and the magnetic dipole moment of the Earth. This relationship can be described by Störmer's equation (e.g., Cooke 1974; Cooke et al. 1991; Stanev 2010; Beer et al. 2012):

$$
P_{\mathrm{c}}=\frac{M}{R^{2}} \frac{\cos ^{4} \lambda_{\mathrm{G}}}{\left[1+\left(1+\cos \theta \cos ^{3} \lambda_{\mathrm{G}}\right)^{1 / 2}\right]^{2}},
$$

where $R$ is the distance from any given location to the dipole centre, $\lambda_{\mathrm{G}}$ is the geomagnetic latitude, $M$ is the geomagnetic dipole moment, and $\theta$ is the angle between the incident ray and the West-East plane (westward). The physical meaning of Eq. (2.10) is that the cosmic rays with rigidity smaller than its cut-off rigidity are not energetic enough to enter the geomagnetic field and are, therefore "blocked" completely from reaching the top of the atmosphere. The Störmer cut-off rigidity approaches zero at geomagnetic latitudes above $65^{\circ}$. In other words, GCRs with almost all energies can reach the Earth surface at high geomagnetic latitudes. Note that the rigidity of the cosmic ray also depends on the incident angle, $\theta$, i.e., $\theta=0^{\circ}$, and $\theta=180^{\circ}$ for the GCRs arriving from the western and eastern horizon, respectively. The rigidity for the GCRs entering from the east is higher than that entering from the west. Therefore, there are more GCRs coming from the west side at the equator. This is the 'East-West asymmetry' effect.

The non-dipolar components of the geomagnetic field are often omitted when computing the cut-off rigidity since the it decays dramatically with increasing distance from the Earth. In addition, when only considering the vertically incident GCRs $\left(\theta=90^{\circ}\right)$, the East-West asymmetry is often ignored. Under these assumptions, Eq. (2.10) can be derived as a vertical cut-off rigidity equation (Smart and Shea 1967, 1985; Beer et al. 2012):

$$
P_{\mathrm{c}}=14.9 \frac{\cos ^{4} \lambda_{\mathrm{G}}}{R^{2}}
$$

The Eq. (2.11) implies that the cosmic rays with a rigidity lower than $14.9 \mathrm{GV}$ is not energetic enough to reach the geomagnetic equator with a vertical incident angle. In contrast, the cut-off rigidity is only $3.7 \mathrm{GV}$ for reaching the geomagnetic latitude of $45^{\circ}$.

As a consequence, it is easy to understand from both Eqs. (2.10) and (2.11) that the changes in the geomagnetic dipole field have no effect on the production rate of cosmogenic radionuclides at high geomagnetic latitudes $\left(>65^{\circ}\right)$ while they have large effect at low geomagnetic latitudes. This effect is the so-called geomagnetic modulation. To conclude with Sect. 2.3.1 that the low energy end of the CR spectrum is contributed by the SEP, which easily reaches the Earth's polar regions owning to the weaker geomagnetic modulation, the SEP can only generate cosmogenic isotopes at high latitudes. 


\subsection{Cosmogenic isotopes}

When GCRs successfully travel through the heliosphere and the geomagnetic field, they finally reach the top of the Earth's atmosphere. These incident GCRs are hence called the primary cosmic rays. When the primary particles interact with the atmospheric nuclei, secondary particles are produced in further interactions. This process is a so-called atmospheric cascade, shown in Fig. 2.6. The cascade process produces a wide variety of secondaries, such as protons $(\mathrm{p})$, neutrons $(\mathrm{n})$, muons $(\mu)$, pions $(\pi)$, electrons $\left(\mathrm{e}^{-}\right)$, positrons $\left(\mathrm{e}^{+}\right)$, and photons $(\gamma)$. Among all, the nucleonic particles ( $\mathrm{n}$ and $\mathrm{p}$ ) dominate the production of cosmogenic (cosmic-ray-produced) isotopes in the atmosphere, while a very small portion of isotopes are produced in soil directly (Lal 1988). Apart from the cosmogenic isotopes produced in the Earth's upper atmosphere, they can also be found in the extraterrestrial solid matter (lunar soil or meteorites) when the materials are exposed to cosmic rays.

The nucleonic and electromagnetic cascades in the atmosphere and the passage of the particles through matter have been extensively simulated by many models using Monte Carlo calculation methods in nuclear physics, such as the MCNP $^{12}$ (Waters et al. 2007; Goorley et al. 2012), the GEANT ${ }^{13}$ (Agostinelli et al. 2003; Allison et al. 2006, 2016; Pshenichnov et al. 2010), the SHIELD-HIT ${ }^{14}$ (e.g., Hultqvist et al. 2012) and the FLUKA ${ }^{15}$ models (e.g., Fasso et al. 1993; Ferrari and Sala 2001; Battistoni et al. 2014).

The connection between the production rate, $Q$, of a cosmogenic isotope at a given location and the cosmic ray flux is given by Solanki et al. (2004) and Usoskin (2017):

$$
Q=\int_{P_{\mathrm{c}}\left(\lambda_{\mathrm{G}}\right)}^{\infty} J(P, \phi) Y(P) d P
$$

where $J(P, \phi)$ is the differential energy spectrum of $\mathrm{CR}$ (Sect. 2.3.1) and $Y(P)$ is the differential yield function of cosmogenic isotope production (i.e., number of ions produced by primary $\mathrm{CR}$ with rigidity, $P_{\mathrm{c}}$ ), which is calculated using a Monte Carlo simulation of the atmospheric cascade process (Kovaltsov et al. 2012; Poluianov et al. 2016).

In this thesis, we are interested in one type of the product of the cascade process: cosmogenic isotopes (or cosmogenic radionuclides). Most of the cosmogenic isotopes are produced from nitrogen $(\mathrm{N})$, oxygen $(\mathrm{O})$ and argon $(\mathrm{Ar})$ since these three nuclei are the main constituents of the Earth's atmosphere. The commonly used natural archives of cosmogenic radionuclides are: corals, ocean/lake sediments, speleothems, ice sheets, loess, trees and peat bogs. The cosmogenic isotopes half-lives range from hours to millions of years depending on the species (e.g., Lal and Peters 1967). For instance, ${ }^{36} \mathrm{Cl},{ }^{26} \mathrm{Al}$ and ${ }^{10} \mathrm{Be}$ have half-lives on the order of a million years, ${ }^{14} \mathrm{C}$ has a half-life of $\approx 5730$ years, ${ }^{44} \mathrm{Ti}$ has a half-life of about 60 years, ${ }^{24} \mathrm{Na}$ and ${ }^{28} \mathrm{Mg}$ have half-lives of only a few hours. Each type of the cosmogenic isotope provides different temporal resolutions, covers different periods and locations, and therefore has its own application based on its physical property and characteristic.

\footnotetext{
${ }^{12}$ Monte Carlo N-Particle code, https: //mcnp . lanl .gov/

${ }^{13}$ GEometry ANd Tracking, http://geant 4 .cern.ch/

${ }^{14}$ http://shieldhit.org/

${ }^{15}$ http://www. fluka.org/fluka.php
} 


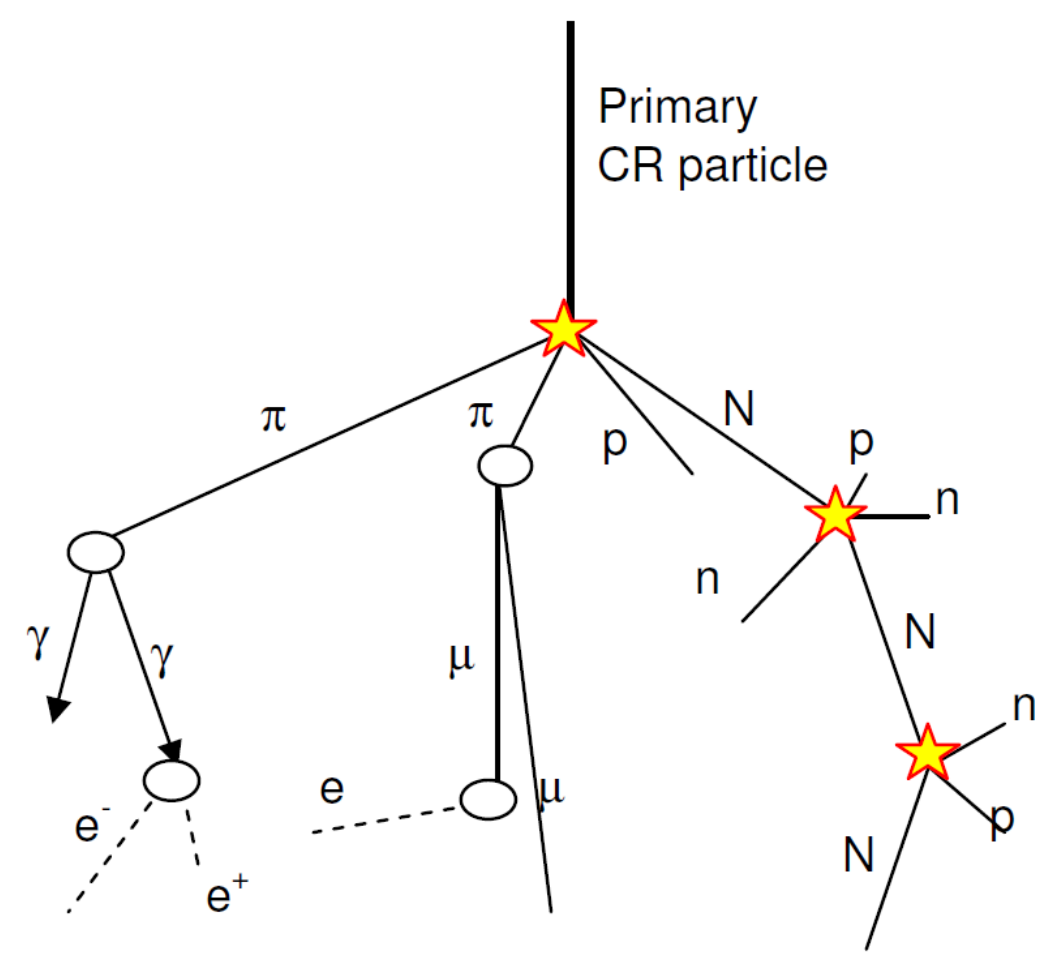

Figure 2.6: Schematic of an atmospheric cascade process. The secondary particles are nuclei $(\mathrm{N})$, protons $(\mathrm{p})$, neutrons $(\mathrm{n})$, muons $(\mu)$, pions $(\pi)$, electrons $\left(\mathrm{e}^{-}\right)$, positrons $\left(\mathrm{e}^{+}\right)$, and photons $(\gamma)$. The stars and circles denote the process of collision and decay, respectively. Taken from Usoskin (2013).

The concentrations of cosmogenic isotopes are often used for reconstructing solar activity (Stuiver and Quay 1980; Stuiver and Braziunas 1989; Bard et al. 1997; Solanki et al. 2004; Muscheler et al. 2007; Steinhilber et al. 2009, 2012; Vieira et al. 2011; Shapiro et al. 2011; Delaygue and Bard 2011; Wu et al. 2018a). Among all the cosmogenic isotopes, ${ }^{14} \mathrm{C}$ and ${ }^{10} \mathrm{Be}$ are mostly widely used to investigate the solar activity on time scales of centuries to millennia due to their abundance in atural archives and their relatively long half-lives. Unfortunately, a large amount of anthropogenic fossil fuel burning in the 20th century and a large scale nuclear weapon tests after the 1950s have heavily contaminated the atmosphere and disturbed the ratio of naturally-produced carbon. The degree of contamination is rather difficult to estimate and the cosmogenic isotope data are therefore not reliable after 1950.

In the following, we will focus on the two most commonly used terrestrial cosmogenic isotopes, ${ }^{14} \mathrm{C}$ and ${ }^{10} \mathrm{Be}$. Their physics background and their production processes are described in Chaps. 2.4.1 and 2.4.2, respectively. 


\subsubsection{Radiocarbon ${ }^{14} \mathrm{C}$}

Carbon has three naturally-produced isotopes. Of these about $98.89 \%$ is ${ }^{12} \mathrm{C}$ and $1.11 \%$ is ${ }^{13} \mathrm{C}$ while ${ }^{14} \mathrm{C}$ is present only in a trace amounts. ${ }^{13} \mathrm{C}$ is a stable isotope while ${ }^{14} \mathrm{C}$ decays radioactively with a half-life of $\approx 5730$ years (Godwin 1962). The ratio between radiocarbon and stable carbon $\left({ }^{14} \mathrm{C} /{ }^{12} \mathrm{C}\right)$ in the atmospheric $\mathrm{CO}_{2}$ is approximately $10^{-12}$ (Stuiver and Polach 1977). Less than $0.1 \%$ of the total amount of ${ }^{14} \mathrm{C}$ is produced in the soil in-situ (Lal 1988; Lal and Jull 2001), implying that this special radionuclide in the terrestrial environment is produced nearly exclusively by GCRs in the upper troposphere and the stratosphere through the following reaction (neutron capture):

$$
\mathrm{n}+{ }_{7}^{14} \mathrm{~N} \rightarrow{ }_{6}^{14} \mathrm{C}+\mathrm{p} .
$$

The existence of ${ }^{14} \mathrm{C}$ was first predicted by Franz Kurie in 1934, and later discovered by Martin Kamen and Sam Ruben in 1940 from sewage methane (Anderson et al. 1947; Libby 1952; Kamen 1963). ${ }^{14} \mathrm{C}$ has been proven to be the most accurate "chronometer" for terrestrial events in the past $\approx 40000$ - 60000 years (Lal and Jull 2001). Therefore, it is widely applied in archaeological/anthropological dating and dendrochronology (e.g., Libby et al. 1949; Libby 1952; Dorman 2004; Kromer 2009).

After ${ }^{14} \mathrm{C}$ is produced in the atmosphere, it is oxidized to ${ }^{14} \mathrm{CO}_{2}$ and mixes with atmospheric $\mathrm{CO}_{2}$ by taking part in the global carbon cycle (e.g., Bolin et al. 1979), which is a coupled atmosphere-biosphere-ocean system, as illustrated in Fig. 2.7. In the biosphere, $\mathrm{CO}_{2}$ is absorbed by trees and grasses, which are later consumed by herbivores. The living bodies therefore have a constant concentration of ${ }^{14} \mathrm{C}$ until they die. After their death, parts of the $\mathrm{CO}_{2}$ returns to the atmosphere while some stay in the soil, entering the geosphere. In addition, atmospheric $\mathrm{CO}_{2}$ can also be dissolved in the ocean, forming calcium carbonate $\left(\mathrm{CaCO}_{3}\right)$, which sinks down to the seafloor and creates sediments. ${ }^{14} \mathrm{C}$ in the sediments may accumulate and sub-duct below the continental plates until they are released back into the atmosphere through volcanic eruptions.

Among all the possible reservoirs mentioned above, tree rings are the most easily assessed and analysed due to their distinguishable annual growth rings. Therefore, a long ${ }^{14} \mathrm{C}$ record can be constructed by counting tree rings and comparing the ring widths among trees covering different regions and periods. This method is called dendrochronology (e.g., Kromer 2009). Due to a large percentage of ocean coverage (80\%) in the southern hemisphere compared to that in the northern hemisphere (60\%), the carbon mixing effect is more effective in the southern hemisphere, and the ${ }^{14} \mathrm{C}$ concentration is, hence, slightly lower $(2-4$ per mille) than the measurements in the north hemisphere. Nevertheless, the measurements of ${ }^{14} \mathrm{C}$ from tree rings show generally a worldwide uniformity, which indicates the global mixing process is rather efficient. Since the global atmospheric circulation varies on seasonal to annual time scales, the time-dependent atmospheric effects (or the so-called "system effects") need to be modelled properly to be distinguished from the "production change" (i.e., the Sun).

The development of a numerical atmospheric cascade model (Fig. 2.6) allows the production rate, $Q$ (in units of [atoms $\left./ \mathrm{sec} / \mathrm{cm}^{2}\right]$ ), to be described as a function of modulation potential, $\phi$, and the geomagnetic dipole moment, $M$ (see Fig. 9 in Usoskin 2013). From this function, the solar magnetic activity can be derived if the geomagnetic dipole moment and the production rate are known. The production rate can be calculated from 


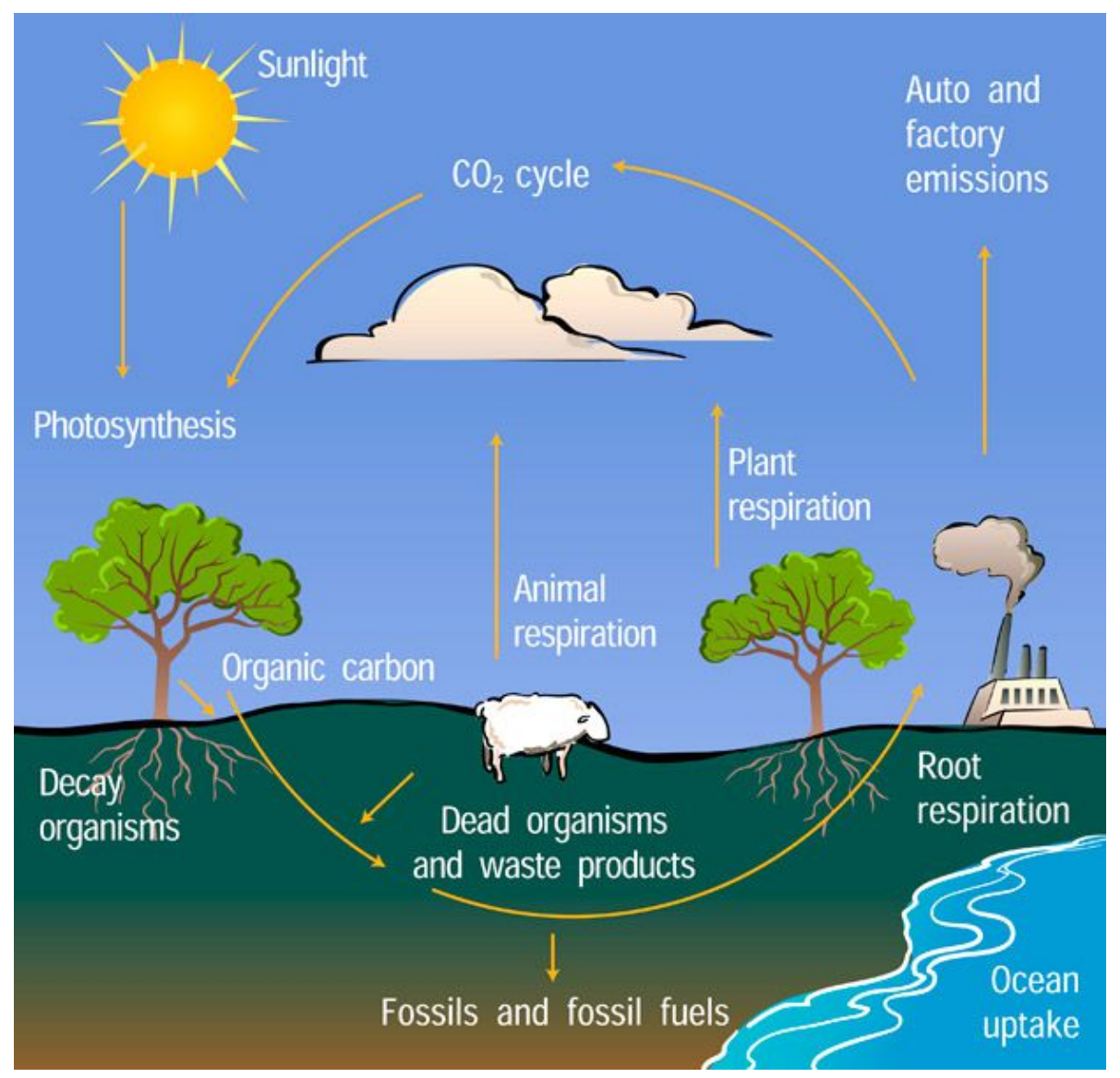

Figure 2.7: Schematic of the global carbon cycle. Five main carbon reservoirs are the atmosphere, the biosphere, the ocean, the sediments and the Earth's crust. The global carbon cycle describes the exchange of carbon between reservoirs through various processes (including chemical, physical, geological, and biological). Courtesy of UCAR.

the concentration of ${ }^{14} \mathrm{C}\left(\Delta^{14} \mathrm{C}\right)$, which is measured in the tree rings. The global carbon cycle is usually described by a box model (see Fig. 2 in Bard et al. 1997), which simulates the fluxes between different carbon reservoirs (such as atmosphere, terrestrial biota, deep sea, mixed ocean layers and marine sediments) as well as the mixing processes within the ocean reservoirs (Oeschger et al. 1975; Siegenthaler et al. 1980; Garcon and Minster 1988; Bard et al. 1997; Hughen et al. 2006).

Complex models, that account for the subdivisions of reservoirs and the interchange between ocean and atmosphere in higher latitude, can achieve detailed and realistic simulations. These models are, however, much more computationally expensive. With interest in calculating the mean production rate from $\Delta^{14} \mathrm{C}$ of the mean reservoirs, a simplified box model is sufficient to represent the global system (Usoskin and Kromer 2005). The globally-averaged production rate is about $1.7-2.3$ atoms $/ \mathrm{sec} / \mathrm{cm}^{2}$ (e.g., O'brien 1979; Masarik and Beer 1999; Goslar 2001; Kovaltsov et al. 2012), which is the largest production rate compared to that of all other cosmogenic radionuclides.

${ }^{14} \mathrm{C}$ was a good indicator of solar activity until the massive emissions of anthropogenic gases and a series of nuclear bomb tests in the mid-20th century. The burning of fossil fuel, which does not contain ${ }^{14} \mathrm{C}$ (since it has decayed long ago), releases significant 
additional amounts of $\mathrm{CO}_{2}$ into the atmosphere. This "artificially-produced" ${ }^{12} \mathrm{C}$ reached a peak in $1963-1965$, thus diluting the atmospheric ${ }^{14} \mathrm{CO}_{2}$ concentration drastically with respect to the pre-industrial epoch. This dilution is known as the Suess effect (Suess 1955; Keeling 1979).

Furthermore, thermonuclear weapon tests between 1950 - 1962 increased the amount of atmospheric thermal neutron and therefore doubled the amount of ${ }^{14} \mathrm{C}$ in the northern hemisphere (Damon et al. 1978; Taylor 1987). This doubled ${ }^{14} \mathrm{C}$ inventory can be seen in both tree rings and the direct measurements in the atmosphere.

These two main anthropogenic effects, unfortunately, affect the accuracy of carbon dating and make the ${ }^{14} \mathrm{C}$ data difficult to be used as a proxy for solar activity after 1950 (Joos 1994). Therefore, the Before Present (BP) time scale is introduced, mainly used in geology and radiocarbon-dating disciplines. BP is defined as the time before $1950 \mathrm{AD}$ (present) to distinguish the epochs before/after the nuclear tests altering the portion of ${ }^{14} \mathrm{C}$ in the atmosphere.

\subsubsection{Radionuclide ${ }^{10} \mathrm{Be}$}

Beryllium has only one stable isotope, ${ }^{9} \mathrm{Be}$, which can be found only in naturally occurring minerals. ${ }^{9} \mathrm{Be}$ has an abundance of $4-6 \mathrm{ppm}$ in the Earth's crust and the uppermost solid mantel. The radioactive isotope ${ }^{10} \mathrm{Be}$ is another commonly used indicator of solar activity on millennial time scales due to its long half-life of about $1.39 \times 10^{6}$ years (Korschinek et al. 2010). It was discovered independently by two groups (Arnold 1956; Goel et al. 1957). Due to its long decay time and very low concentration, ${ }^{10} \mathrm{Be}$ can be precisely measured only by the Accelerator Mass Spectrometry (AMS) technique invented in the 1980s. This technique achieves an accuracy (ratio of ${ }^{10} \mathrm{Be} /{ }^{9} \mathrm{Be}$ ) better than $10^{-13} \cdot{ }^{10} \mathrm{Be}$ is originally measured in concentration (in units of [atoms/g]) and is often converted into production rate or flux (in units of [atoms $\left./ \mathrm{sec} / \mathrm{cm}^{2}\right]$ ) if possible.

$99.9 \%$ of ${ }^{10} \mathrm{Be}$ is produced in the terrestrial atmosphere through cosmic ray spallation while the remaining $0.1 \%$ is from the in-situ production when the GCRs-induced secondary particles interact with mineral/rock surfaces. In the atmospheric cascade, ${ }^{10} \mathrm{Be}$ can be produced through multiple reactions as the following:

$$
\begin{gathered}
\mathrm{n}+{ }_{7}^{14} \mathrm{~N} \rightarrow{ }_{4}^{10} \mathrm{Be}+3 \mathrm{p}+2 \mathrm{n}, \\
\mathrm{p}+{ }_{7}^{14} \mathrm{~N} \rightarrow{ }_{4}^{10} \mathrm{Be}+4 \mathrm{p}+\mathrm{n}, \\
\mathrm{n}+{ }_{8}^{16} \mathrm{O} \rightarrow{ }_{4}^{10} \mathrm{Be}+4 \mathrm{p}+3 \mathrm{n}, \\
\mathrm{p}+{ }_{8}^{16} \mathrm{O} \rightarrow{ }_{4}^{10} \mathrm{Be}+5 \mathrm{p}+2 \mathrm{n} .
\end{gathered}
$$

Similar to ${ }^{14} \mathrm{C}$, the production rate of ${ }^{10} \mathrm{Be}$ can be described as a function of modulation potential and the geomagnetic dipole moment (see Fig. 3 in Vonmoos et al. 2006). The total energy of the cascade (increases with altitude) and the the number of secondary particles (decrease with altitude) balance each other at an altitude of $10-15 \mathrm{~km}$, where most of the global ${ }^{10} \mathrm{Be}$ (about $55-70 \%$ ) is produced (e.g., Lal and Peters 1967; Masarik and Beer 1999, 2009; Usoskin and Kovaltsov 2008; Kovaltsov and Usoskin 2010).

In contrast to ${ }^{14} \mathrm{C}$, which participates in the global circulation, ${ }^{10} \mathrm{Be}$ takes a different geochemical path after its production. After the radionuclide is produced, ${ }^{10} \mathrm{Be}$ becomes attached to the atmospheric aerosols, such as sulfates, sea salts and ammonium salts. 
Since aerosols often act as $\mathrm{CCN},{ }^{10} \mathrm{Be}$ can thereby easily fall out to the Earth's surface by either wet or dry deposition. The wet deposition is an efficient and precipitation-related process, which includes all processes that involve water in many forms (e.g., rain, snow, hail). The dry deposition involves turbulence and the ${ }^{10} \mathrm{Be}$ is removed from the atmosphere when it touches and adheres to any surface, such as soil and vegetation. Compared to the dry deposition, which is only significant in very dry regions, the wet deposition is the main mechanism removing ${ }^{10} \mathrm{Be}$ from the atmosphere.

The signal of ${ }^{10} \mathrm{Be}$ can be found in many types of water-related reservoirs, such as oceans, glaciers, lake sediments and polar ice (e.g., Beer et al. 1988; Wieland et al. 1991). Among all these, ice cores are the most commonly-employed resource for studying the long-term solar activity on the basis of ${ }^{10} \mathrm{Be}$ concentration. Although the ice cores have the traceable layers, it is difficult to date them correctly especially with increasing depth. This is due to the thinning effect of the ice, which describes the ice annual thickness decreasing logarithmically with increasing depth owing to the increasing pressure of the ice.

The aerosol-bound ${ }^{10} \mathrm{Be}$ in the stratosphere has a residence time of about $1-2$ years while the ${ }^{10} \mathrm{Be}$ in the troposphere has a short residence time of about a few weeks (Raisbeck et al. 1981; Masarik and Beer 1999). Therefore, the ${ }^{10} \mathrm{Be}$ in the stratosphere could be slightly atmospheric-mixed compared to the ${ }^{10} \mathrm{Be}$ in the troposphere (Raisbeck et al. 1981; Masarik and Beer 1999). Generally, the sampled polar ice cores have a greater proportion ${ }^{10} \mathrm{Be}(60-64 \%)$ from the stratosphere, with only $20-22 \%$ from the polar region troposphere, and less than $16-18 \%$ from the troposphere at mid-latitude (Beer et al. 2012). The opposite hemisphere and the equatorial region have negligible effects on the polar ice. Additional evidence for the ${ }^{10} \mathrm{Be}$ signal being highly climate-influenced is the halved ${ }^{10} \mathrm{Be}$ concentrations during the Holocene compared to that in the last glacial period. This is attributed to the fact that the water cycle was reduced during the last glacial period, causing a lower precipitation rate in the polar regions $(\approx 50 \%$ of today's value), and resulting in an approximately doubled ${ }^{10} \mathrm{Be}$ concentration. Consequently, the measured ${ }^{10} \mathrm{Be}$ concentrations in the polar ice contain both production signal (solar activity) and the local climatic/precipitation signals (e.g., Steig et al. 1996; Bard et al. 1997). The common solar signal can be qualitatively observed by comparing the ${ }^{10} \mathrm{Be}$ series from Greenland and Antarctica with the globally-mixed ${ }^{14} \mathrm{C}$ series.

Since the concentration of ${ }^{10} \mathrm{Be}$ is subjected to the local climate due to partial atmospheric mixing (Kocharov et al. 1989; McHargue and Damon 1991), the transport process has to be taken into account when converting the concentration in natural archives to the production rate in the atmosphere. The computations and models of ${ }^{10} \mathrm{Be}$ production have been developed since the 1960s, with various approaches and numerical methods (Bhandari et al. 1966; Lal and Peters 1967; Lal and Suess 1968; O'brien 1979; Masarik and Beer 1999). Modern atmospheric 3-D general circulation models (e.g., the NASA GISS model; Field et al. 2006) for simulating the air-mass transport and wet/dry deposition processes (e.g., Field et al. 2006; Heikkilä et al. 2009) allow one to estimate the local climatic effects. The models, that are based on full Monte Carlo simulations of atmospheric cascades, show the globally-averaged production rate of ${ }^{10} \mathrm{Be}$ is about $0.02-$ 0.03 atoms $/ \mathrm{sec} / \mathrm{cm}^{2}$ (Webber and Higbie 2003; Webber et al. 2007; Usoskin et al. 2006b; Usoskin and Kovaltsov 2008; Kovaltsov and Usoskin 2010). It is about two orders of magnitude smaller than that of ${ }^{14} \mathrm{C}$ (Sect. 2.4.1) because most of the neutrons produced in 


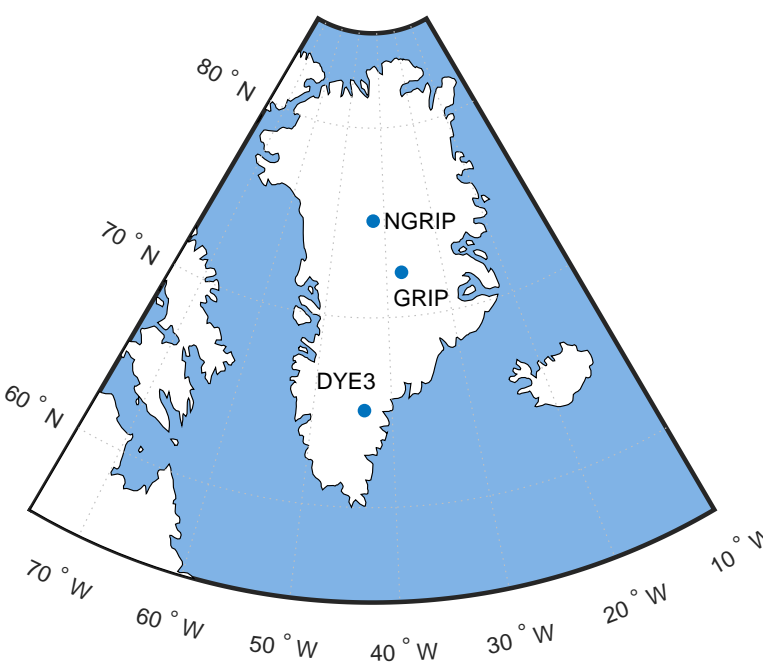

(a) Greenland

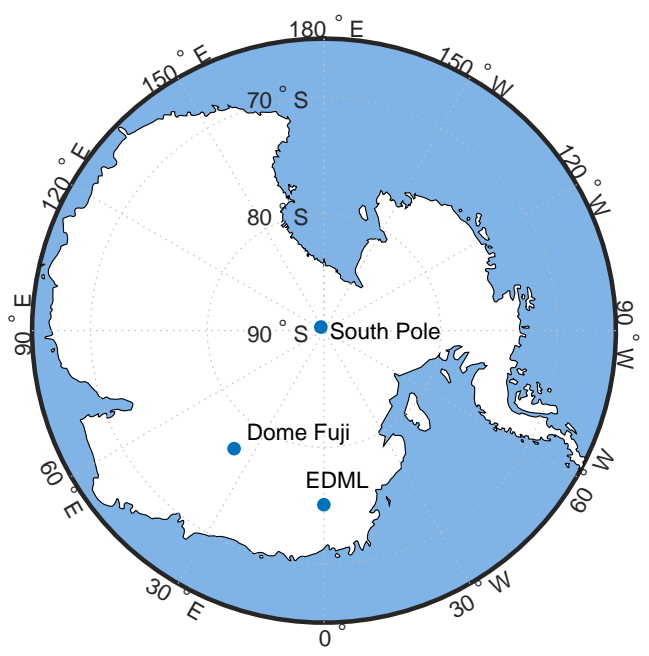

(b) Antarctica

Figure 2.8: The drilling sites of the $\operatorname{six}{ }^{10} \mathrm{Be}$ series used in this thesis.

the cascade process end up producing the ${ }^{14} \mathrm{C}$ (through neutron capture), and only those neutrons and protons with energy $>10 \mathrm{MeV}$ can further produce ${ }^{10} \mathrm{Be}$.

The validity of the models has been demonstrated by Usoskin et al. (2009) and Pedro et al. (2011). However, the choice of transportation models can result in different solar modulation potentials, $\phi$, for the same measured ${ }^{10} \mathrm{Be}$ value. Two extreme scenarios, which assume either: (1) ${ }^{10} \mathrm{Be}$ is only produced at high latitudes or, (2) ${ }^{10} \mathrm{Be}$ is only globally-mixed, have been tested to be unrealistic (i.e., they either over or underestimate the solar activity). The actual scenario should be somewhere in between these two extrema.

There have been numerous ice cores drilled at different locations in both Greenland and Antarctica, such as Camp Century, Greenland (Beer et al. 1988), GRIP, Greenland (Yiou et al. 1997), Dye 3, Greenland (Beer et al. 1990), GISP2, Greenland (Finkel and Nishiizumi 1997), Milcent, Greenland (Beer et al. 1983), NGRIP, Greenland (DahlJensen et al. 2002; Hvidberg et al. 2002; Berggren et al. 2009), Dome C (Concordia), Antarctica (Raisbeck et al. 1981), South Pole, Antarctica (Raisbeck et al. 1990; Bard et al. 1997), Dome Fuji, Antarctica (Horiuchi et al. 2007), EPICA Dronning Maud Land (EDML), Antarctica (Ruth et al. 2007; Steinhilber et al. 2012). Different records from these sites provide different temporal resolutions and cover different periods of time. In this thesis, we use three ${ }^{10}$ Be series from Greenland (GRIP, NGRIP and Dye3) and three from Antarctic (EDML, Dome Fuji and South Pole). Their locations are shown in the Fig. 2.8 . 


\title{
3 Solar activity over nine millennia: A consistent multi-proxy reconstruction
}

\author{
*This chapter is published as a journal article "Solar activity over nine millennia: A con- \\ sistent multi-proxy reconstruction" (DOI: 10.1051/0004-6361/201731892) in Astronomy \\ \& Astrophysics. \\ Credit: C.-J Wu ${ }^{1,2}$, I. G. Usoskin ${ }^{3,4}$, N. A. Krivova ${ }^{1}$, G. A. Kovaltsov ${ }^{5}$, M. Baroni ${ }^{6}$, E. \\ Bard $^{6}$ and S. K. Solanki ${ }^{1,7}$, A\&A, 2018, reproduced with permission (C) ESO.
}

\begin{abstract}
Aims. Solar activity in the past millennia can only be reconstructed from cosmogenic radionuclide proxy records in terrestrial archives. However, because of the diversity of the proxy archives, it is difficult to build a homogeneous reconstruction. All previous studies were based on individual, sometimes statistically averaged, proxy datasets. Here we aim to provide a new consistent multi-proxy reconstruction of solar activity over the last 9000 years, using all available long-span datasets of ${ }^{10} \mathrm{Be}$ and ${ }^{14} \mathrm{C}$ in terrestrial archives.

Methods. A new method, based on a Bayesian approach, was applied for the first time to solar activity reconstruction. A Monte-Carlo search (using the $\chi^{2}$ statistic) for the most probable value of the modulation potential was performed to match data from different datasets for a given time. This provides a straightforward estimate of the related uncertainties. Six ${ }^{10} \mathrm{Be}$ series of different lengths (from $500-10000$ years) from both Greenland and Antarctica, as well as the global ${ }^{14} \mathrm{C}$ production series were used. The ${ }^{10} \mathrm{Be}$ series were re-sampled to match wiggles, related to the Grand minima, in the ${ }^{14} \mathrm{C}$ reference dataset. Stability of the long data series was tested.

Results. The Greenland GRIP (Greenland Ice-core Project) and Antarctic EDML (EPICA Dronning Maud Land) ${ }^{10} \mathrm{Be}$ series diverge from each other during the second half of the Holocene, while the ${ }^{14} \mathrm{C}$ series lies in between them. A likely reason for the discrepancy is the insufficiently precise beryllium transport and deposition model for Greenland, which leads to under-correction of the GRIP series for the geomagnetic shielding effect. A slow 6

\footnotetext{
${ }^{1}$ Max-Planck-Institut für Sonnensystemforschung, Justus-von-Liebig-Weg 3, Göttingen, Germany

${ }^{2}$ Georg-August-Universität Göttingen, Institute for Astrophysics, Göttingen, Germany

${ }^{3}$ Space Climate Research Unit, University of Oulu, Finland

${ }^{4}$ Sodankylä Geophysical Observatory, University of Oulu, Finland

${ }^{5}$ Ioffe Physical-Technical Institute, 194021 St. Petersburg, Russia

${ }^{6}$ CEREGE, Aix-Marseille University, CNRS, Collège de France, Technopôle de l'Arbois, Aix-enProvence, France

${ }^{7}$ School of Space Research, Kyung Hee University, Yongin, Gyeonggi-Do,446-701, Republic of Korea
} 
-7-millennia variability with lows ca. $5500 \mathrm{BC}$ and $1500 \mathrm{AD}$ in the long-term evolution of solar activity is found. Two components of solar activity can be statistically distinguished: the main component, corresponding to the 'normal' moderate level, and a component corresponding to Grand minima. A possible existence of a component representing Grand maxima is hinted, but it cannot be separated from the main component in a statistically significant manner.

Conclusions. A new consistent reconstruction of solar activity over the last nine millennia is presented with the most probable values of decadal sunspot numbers and their realistic uncertainties. Independent components of solar activity corresponding to the main moderate activity and the grand-minimum state are identified, possibly related to different modes of the operation of the dynamo.

\subsection{Introduction}

The Sun is an active star whose magnetic activity varies on different time scales, from seconds to millennia. Understanding solar variability in detail is important for many reasons, ranging from applications in stellar astrophysics and dynamo theory to paleoclimatic and space weather studies. Various direct, partly multi-wavelength spectroscopic solar observations cover the past few decades up to a century in the past. These provide knowledge of the solar variability expressed in different indices. For the preceding centuries back to 1610, only visual information from simple optical observations in the form of sunspot numbers is available (Hathaway 2015). The quality of the sunspot number series is uneven and the uncertainty increases sufficiently before the 19th century (Clette et al. 2014; Usoskin 2017). Yet the Maunder minimum in the second half of the 17th century was observed sufficiently good to conclude that sunspot activity was exceptionally low (Ribes and Nesme-Ribes 1993; Vaquero et al. 2015; Usoskin et al. 2015). For yet earlier times, only indirect proxies can help assessing solar activity ${ }^{1}$. Such proxies are, for example, concentrations of cosmogenic radionuclides radiocarbon $\left({ }^{14} \mathrm{C}\right)$, beryllium-10 $\left({ }^{10} \mathrm{Be}\right)$ or chlorine-36 $\left({ }^{36} \mathrm{Cl}\right)$ measured in tree trunks or polar ice cores, respectively. These archives are dated independently. The use of cosmogenic proxies to study solar activity in the past has been proposed long ago (e.g., Stuiver 1961; Stuiver and Quay 1980; Beer et al. 1988), and the method has been developed since then in both measurements and modelling (see reviews by Beer et al. 2012; Usoskin 2017, and references therein). Such data cover time scales up to ten millennia and more.

Cosmogenic radionuclides are produced as a by-product of a nucleonic cascade initiated by galactic cosmic rays (GCR) in the Earth's atmosphere. This is the only source of these nuclides in the terrestrial system (which is why they are called "cosmogenic"). Radiocarbon ${ }^{14} \mathrm{C}$ is mainly produced as a result of neutron capture ( $n p$-reaction) by nitrogen which is responsible for $>99 \%$ of the natural ${ }^{14} \mathrm{C}$ production. The isotope ${ }^{10} \mathrm{Be}$ is produced as a result of spallation reactions of $\mathrm{O}$ and $\mathrm{N}$ nuclei caused by energetic GCR particles. After production, the two radionuclides have different processes of transport, deposition and storage in terrestrial archives around the globe. Radiocarbon is mostly measured in dendrochronologically dated annual rings of live or dead tree trunks, while ${ }^{10} \mathrm{Be}$

\footnotetext{
${ }^{1}$ Although naked-eye observations of sunspots are available, they do not provide quantitative assessments (Usoskin 2017).
} 
is measured in glacilogically dated polar ice cores mostly from Greenland or Antarctica. In addition to the geomagnetic field, the flux of GCR impinging on Earth is modulated by large-scale heliospheric magnetic features (interplanetary magnetic fields and solar wind) so that the measured content of the nuclides may serve as a proxy of solar magnetic variability in the past (once the influence of the geomagnetic field has been removed). Different cosmogenic isotope series exhibit a high degree of similarity on time scales of a century to a millennium, due to the same production origin (Bard et al. 1997; Vonmoos et al. 2006; Beer et al. 2012; Usoskin et al. 2009; Delaygue and Bard 2011; Usoskin et al. 2016a; Adolphi and Muscheler 2016). However, systematic discrepancies between longterm (multi-millennial) trends in different series can be observed (Vonmoos et al. 2006; Inceoglu et al. 2015; Adolphi and Muscheler 2016; Usoskin et al. 2016a), probably due to the influence of climate conditions (regional deposition pattern for ${ }^{10} \mathrm{Be}$ or large-scale ocean circulation for ${ }^{14} \mathrm{C}$ ) or an improper account for the geomagnetic field variation to which the two isotopes respond differently.

Because of the differences in the isotope records, earlier reconstructions of solar activity were obtained based on individual cosmogenic series, leading to a diversity in the results. Earlier multi-proxy efforts (e.g., Bard et al. 1997; McCracken et al. 2004; Vonmoos et al. 2006; Muscheler et al. 2007; Usoskin et al. 2007; Knudsen et al. 2009) were mostly based on a simple comparison of individual records. The first consistent effort to produce a merged reconstruction was made by Steinhilber et al. (2012) who used the principle component analysis (PCA) to extract the common variability signal (assumed to be solar) from the reconstructions based on three cosmogenic series (global ${ }^{14} \mathrm{C}$, Antarctic EDML (EPICA Dronning Maud Land) and Greenland GRIP (Greenland Ice-core Project) ${ }^{10} \mathrm{Be}$ ones) and to remove the system effects (e.g., the deposition process, snow accumulation rate and changes in the carbon cycle and dating uncertainties) which are different for each series. The PCA method keeps only the relative variability, losing the information on the absolute level, which needs further normalization. Moreover, this method effectively averages multiple signals without taking the accuracy of each data point and possible time lags between the signals into account (see Figures S1 - S8 in Steinhilber et al. 2012). As discussed by Adolphi and Muscheler (2016), the time mismatch between ${ }^{14} \mathrm{C}$ and ${ }^{10} \mathrm{Be}$ series may, however, be as large as 70 years towards the early Holocene.

In this work, we introduce a new method of consistent multi-proxy reconstruction of solar activity, based on the Bayesian approach to find the most probable value (and its uncertainties) of solar activity at any moment of time by minimization of the $\chi^{2}$-discrepancy between the modelled and the actually measured cosmogenic isotope data. This method straightforwardly accounts for error propagation and provides the most probable reconstruction and its realistic uncertainties. Since the method is sensitive to the dating accuracy of different records, we re-dated the ${ }^{10} \mathrm{Be}$ records to match their dating with that of ${ }^{14} \mathrm{C}$, using the standard wiggle-matching method (Cain and Suess 1976; Muscheler et al. 2014) applied to the official ice-core chronology, as described in Section 3.3. The solar modulation potential was assessed using the Bayesian approach from all the available datasets (Section 3.4). Finally, the sunspot number series was calculated (Section 3.5). Section 3.6 summarizes our results and conclusions. 


\subsection{Data}

\subsubsection{Cosmogenic isotope records}

In this work, we use six ${ }^{10} \mathrm{Be}$ series, from both Greenland and Antarctica and one global

${ }^{14} \mathrm{C}$ production series as summarized in Table 3.1 and Figure 3.1.

The ${ }^{14} \mathrm{C}$ series covers the entire Holocene with homogeneous resolution as given by the globally averaged ${ }^{14} \mathrm{C}$ production rate computed by Roth and Joos (2013) from the original INTCAL09 (International ${ }^{14} \mathrm{C}$ Calibration dataset, Reimer et al. 2009) measurements of $\Delta^{14} \mathrm{C}$. While this series has a pseudo-annual temporal sampling (Roth and Joos 2013), its true resolution is decadal. The series is provided as an ensemble of 1000 realizations of individual reconstructions. Each realization presents one possible reconstruction in the sense of a Monte-Carlo approach (viz. one realized path among all possible ones in the parametric space), considering all known uncertainties. This makes it possible to directly assess error propagation throughout the entire process.

${ }^{10} \mathrm{Be}$ series have different coverage and temporal resolutions. We reduced them to two cadences. Annual series were kept as they are while rougher resolved series were re-sampled to decadal cadence. For each series we also considered 1000 realizations synthesized using the mean curve and the standard deviation (error bars). Two series have been updated with respect to earlier studies. The EDML series has been used with the new AICC2012 (Antarctic Ice Core Chronology, v. 2012) chronology (Veres et al. 2013; Bazin et al. 2013). The GRIP series has been updated from its original ss09(sea) time scale (Johnsen et al. 1995, 2001) to a more recent GICC05 (Greenland Ice Core Chronology, v. 2005) timescale (Vinther et al. 2006).

All ${ }^{10} \mathrm{Be}$ series, when possible, were converted into units of production/flux [atoms $\mathrm{cm}^{-2} \mathrm{sec}^{-1}$ ] which is natural for the isotope production by cosmic rays. Originally, ${ }^{10} \mathrm{Be}$ measurements are given in units of concentration [atoms $\mathrm{g}^{-1}$ ]. To convert them into the flux, the independently measured/obtained snow accumulation rate at each site is needed. The accumulation rate was considered individually for each ice core, using the same chronology as the ${ }^{10} \mathrm{Be}$ data (Veres et al. (2013); Bazin et al. (2013) for EDML on AICC2012 and Rasmussen et al. (2014); Seierstad et al. (2014) for GRIP on the GICC05 timescale). For some series, Dye-3 and South Pole, accumulation data are not provided, and in such cases we used concentration data assuming that the concentration is proportional to the depositional flux, with the scaling factor being a free parameter (Table 3.1).

\subsubsection{Geomagnetic data}

As the geomagnetic data for the last millennia we used a recent archeo/paleomagnetic model GMAG.9k of the virtual axial dipole moment (VADM) as published by Usoskin et al. (2016a) for the period since ca. 7000 BC. This model provides an ensemble of 1000 individual VADM reconstructions including all the uncertainties. The range of the ensemble reconstruction covers other archeo/paleomagnetic models (e.g., Genevey et al. 2008; Knudsen et al. 2008; Licht et al. 2013; Nilsson et al. 2014; Pavón-Carrasco et al. 2014), as can be seen in Figure 2 of Usoskin et al. (2016a), thus covering the related uncertainties. The length of the geomagnetic series limits our study to the period since 6760 BC. 


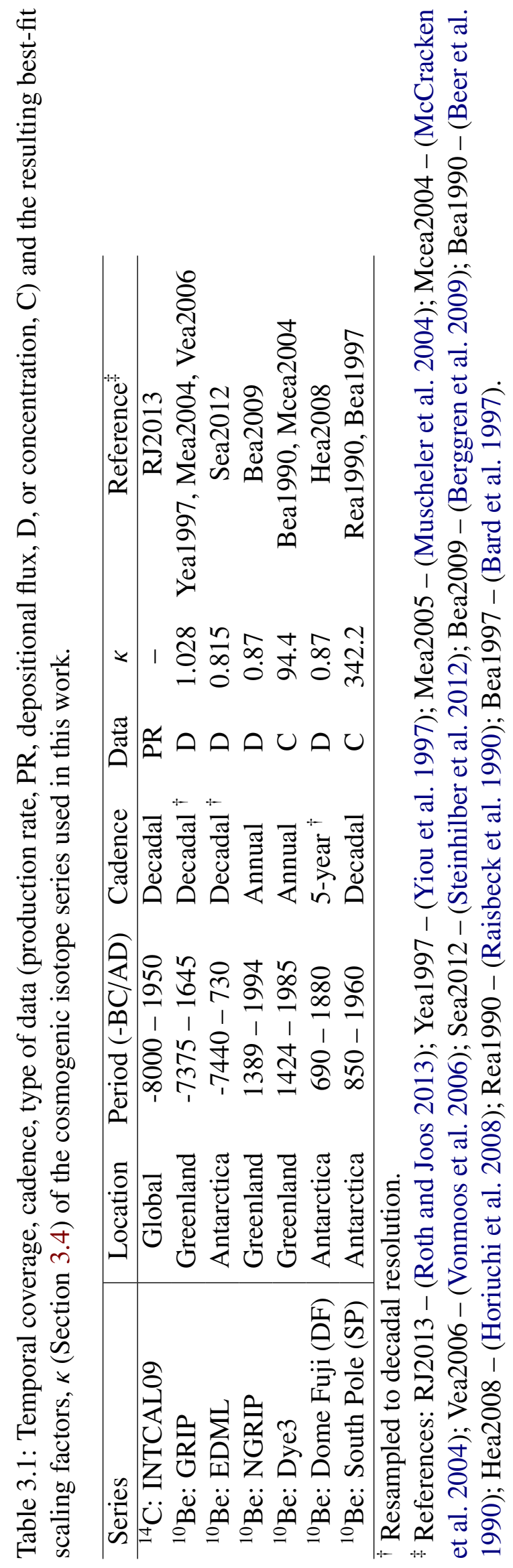




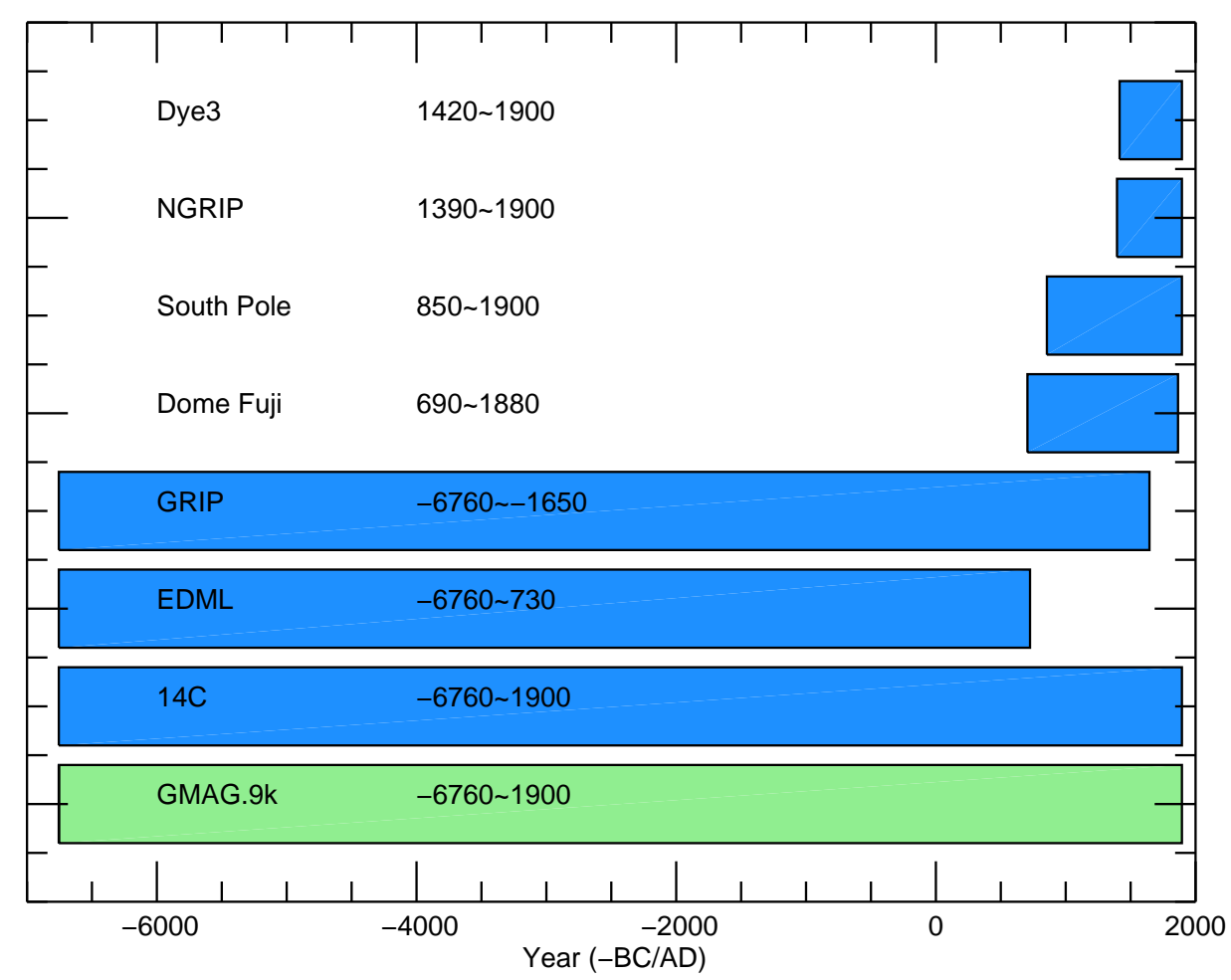

Figure 3.1: Data series used in this work (see Table 3.1) and their temporal coverage, used here. Blue shading denotes the cosmogenic isotope series, while the green one is used for the geomagnetic series employed here.

\subsection{Data processing}

\subsubsection{Temporal synchronization of the records: Wiggle matching}

While the radiocarbon is absolutely dated via dendrochronology by tree ring counting, dating of ice cores is less precise. In principle, dating of ice cores can be done, especially on short timescales, by counting annual layers of sulfate or sodium, if the accumulation and the characteristics of the site allow it (e.g., Sigl et al. 2015). But in practise dating of long series is performed by applying ice-flow models between tie points related to known events like volcano eruptions that leave clear markers in ice. While the accuracy of dating is quite good around the tie points, exact ages of samples between the tie points may be rather uncertain, from several years during the last millennium and up to $70-100$ years in the earlier part of the Holocene (Muscheler et al. 2014; Sigl et al. 2015; Adolphi and Muscheler 2016).

Since our method is based on the minimization of the $\chi^{2}$-statistics among all series for a given moment of time, it is crucial that these series are well synchronized. Accordingly, we performed a formal synchronization of the series based on the wiggle matching procedure, which is a standard method to synchronize time series (e.g., Cain and Suess 
Table 3.2: Wiggles (central date and the length in years) used for synchronization of the various ${ }^{10}$ Be time series to ${ }^{14} \mathrm{C}$ (Eddy 1976; Stuiver and Quay 1980; Stuiver and Braziunas 1989; Goslar 2003; Inceoglu et al. 2015; Usoskin et al. 2016a), as well as the synchronization time (in years) for GRIP and EDML series. All dates are given in -BC/AD for dendrochronologically dated ${ }^{14} \mathrm{C}$.

\begin{tabular}{cc|cc|cc|cc}
\hline Date & Length & $d T$ (EDML) & $d T$ (GRIP) & Date & Length & $d T$ (EDML) & $d T$ (GRIP) \\
\hline 1680 & 80 & N/A & N/A & -3325 & 90 & $-20_{-11}^{+10}$ & $7_{-7}^{+6}$ \\
1480 & 160 & N/A & $8_{-6}^{+5}$ & -3495 & 50 & $-23_{-7}^{+6}$ & $6_{-6}^{+6}$ \\
1310 & 80 & N/A & $2_{-5}^{+4}$ & -3620 & 50 & $-26_{-10}^{+21}$ & $5_{-6}^{+6}$ \\
1030 & 80 & N/A & $0_{-4}^{+4}$ & -4030 & 100 & $-19_{-6}^{+6}$ & $2_{-4}^{+5}$ \\
900 & 80 & N/A & $13_{-3}^{+4}$ & -4160 & 50 & $-22_{-6}^{+5}$ & $-3_{-5}^{+5}$ \\
690 & 100 & $16_{-6}^{+8}$ & $-1_{-5}^{+5}$ & -4220 & 30 & $-24_{-5}^{+5}$ & $-6_{-5}^{+5}$ \\
260 & 80 & $12_{-6}^{+7}$ & $0_{-4}^{+5}$ & -4315 & 50 & $-26_{-5}^{+4}$ & $4_{-3}^{+3}$ \\
-360 & 120 & $6_{-6}^{+5}$ & $2_{-3}^{+6}$ & -5195 & 50 & $-19_{-5}^{+4}$ & $7_{-5}^{+5}$ \\
-750 & 70 & $-18_{-9}^{+5}$ & $0_{-5}^{+2}$ & -5300 & 50 & $-18_{-5}^{+4}$ & $7_{-5}^{+5}$ \\
-1385 & 80 & $-25_{-7}^{+9}$ & $3_{-5}^{+4}$ & -5460 & 40 & $-22_{-5}^{+5}$ & $18_{-4}^{+5}$ \\
-1880 & 80 & $-30_{-6}^{+7}$ & $5_{-6}^{+6}$ & -5610 & 40 & $-36_{-4}^{+9}$ & $29_{-8}^{+7}$ \\
-2120 & 40 & $-27_{-6}^{+5}$ & $6_{-6}^{+7}$ & -5970 & 60 & $-29_{-8}^{+14}$ & $20_{-6}^{+6}$ \\
-2450 & 40 & $-28_{-5}^{+4}$ & $0_{-3}^{+4}$ & -6060 & 60 & $-27_{-9}^{+13}$ & $15_{-8}^{+6}$ \\
-2570 & 100 & $-29_{-4}^{+4}$ & $0_{-3}^{+3}$ & -6385 & 130 & $-22_{-11}^{+10}$ & $-4_{-14}^{+6}$ \\
-2855 & 90 & $-20_{-6}^{+7}$ & $-14_{-10}^{+16}$ & -6850 & 100 & $-19_{-7}^{+7}$ & $-24_{-7}^{+8}$ \\
-3020 & 60 & $-18_{-8}^{+6}$ & $-7_{-9}^{+12}$ & -7030 & 100 & $-25_{-6}^{+6}$ & $-39_{-12}^{+8}$ \\
-3080 & 60 & $-32_{-6}^{+6}$ & $-4_{-9}^{+11}$ & -7150 & 100 & $-29_{-5}^{+5}$ & $-49_{-15}^{+8}$ \\
\hline
\end{tabular}

1976; Hoek and Bohncke 2001; Muscheler et al. 2014). We took the ${ }^{14} \mathrm{C}$ chronology as the reference and adjusted the timing of all other series to that.

\subsubsection{Choice of wiggles}

Since cosmogenic isotope series exhibit strong fluctuations, of various possible origins, it is important to match only those wiggles that can presumptively be assigned to the production changes, viz. cosmic ray (solar) variability and excluding possible regional climate spells. One well suited type of wiggles is related to the so-called Grand minima of solar activity, which are characterized by a fast and pronounced drop of solar activity for several decades. The most famous example of a Grand minimum is the Maunder minimum between 1645 - 1715 (Eddy 1976; Usoskin et al. 2015). Grand minima can be clearly identified in the cosmogenic isotope records as sharp spikes (Usoskin et al. 2007; Inceoglu et al. 2015). A typical (though not the most pronounced) example of such wiggles (Grand minima) is shown in Figure 3.2 for the (scaled) original EDML ${ }^{10} \mathrm{Be}$ series in dashed curve along with the ${ }^{14} \mathrm{C}$ production data in red. Although the overall variability of the two series looks alike, there is a clear mismatch in the timing between them of roughly few decades.

For further analysis we have selected periods with clear wiggles (spikes, corresponding to Grand minima, as listed in earlier works - see references in the notes to Table 3.2) in the ${ }^{14} \mathrm{C}$ series as listed in Table 3.2, along with their centres and time span. 


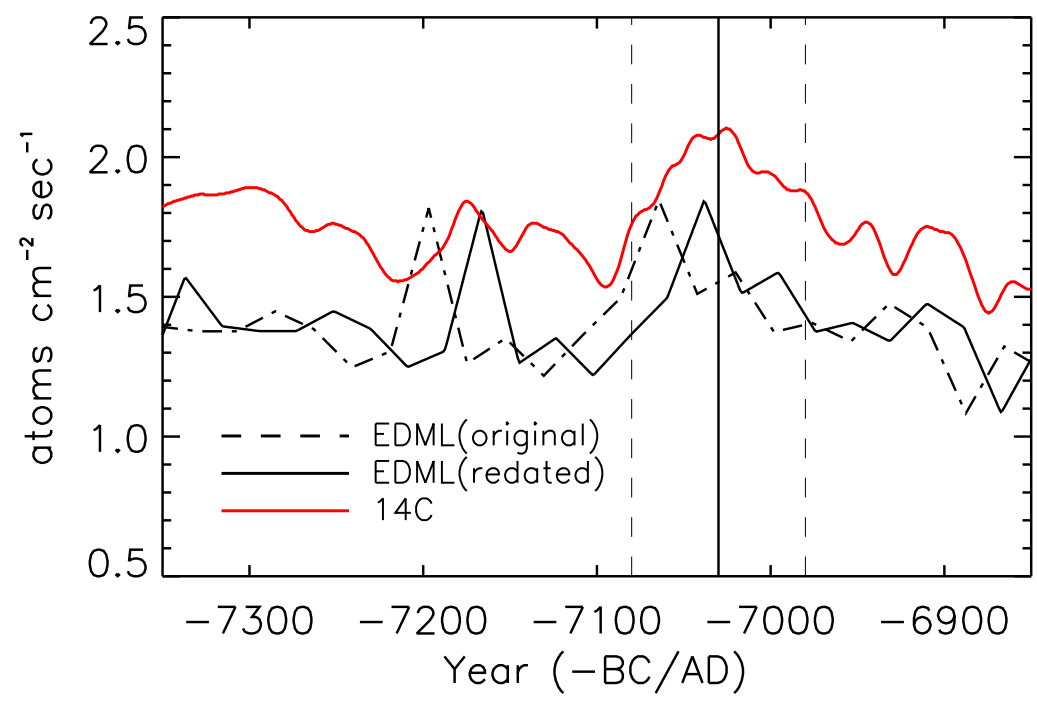

Figure 3.2: A typical example of a wiggle in the ${ }^{14} \mathrm{C}$ (red) series compared with the original EDML ${ }^{10} \mathrm{Be}$ series (dashed black, scaled up by a factor of 172). Solid and dashed vertical lines denote the middle and the span of the wiggle considered for ${ }^{14} \mathrm{C}$. The black sold curve is EDML ${ }^{10} \mathrm{Be}$ series after the synchronization (see Section 3.3.1.2).

\subsubsection{Synchronization of the wiggles}

For all the selected wiggles (Table 3.2), we found the best-fit time adjustment $d T$ between the analysed ${ }^{10} \mathrm{Be}$ and the reference ${ }^{14} \mathrm{C}$ production series by maximizing the cross correlation between the series, calculated within a time window centred at the middle of the wiggle. The data were annually interpolated within the time windows so that the time step in defining $d T$ was 1 year. The length of the correlation window was chosen as the double length of the wiggle (see Table 3.2). For each wiggle, we calculated the Pearson's linear correlation coefficients between ${ }^{14} \mathrm{C}$ and ${ }^{10} \mathrm{Be}$ series repeatedly and the value of $d T$ was selected that maximized the cross-correlation coefficient $R$ between the two series. The standard error $\left(s_{\mathrm{err}}\right)$ of the correlation coefficient was calculated using the approximate formula (e.g., Cohen 2003):

$$
s_{\mathrm{err}}=\sqrt{\frac{1-R_{\mathrm{c}}^{2}}{n-2}}
$$

where $R_{\mathrm{c}}$ is the maximum correlation coefficient and $n$ is the number of the data points within the correlation window. This uncertainty $s_{\text {err }}$ was translated into the $1 \sigma$ confidence interval for $d T$, as illustrated in Figure 3.3 which shows the correlation coefficient, $R$ (black curve), as a function of the time shift $d T$. It reaches its maximum $R_{\mathrm{c}}=0.86$ at $d T=-25$ years, as indicated by the vertical solid line. The dotted lines bound the $68 \%$ confidence interval for $d T$ defined as $R=R_{\mathrm{c}}-s_{\mathrm{err}}$.

The 'momentary' time adjustments $d T$ were considered as 'tie points' (listed in Table 3.2) for the ${ }^{10} \mathrm{Be}$ series, with a linear interpolation used between them. One can see that adjustments for the EDML series, based here on a new Antarctic Ice Core Chronology (AICC2012; Veres et al. 2013), lie within +20/-40 years, while for the GRIP series they 


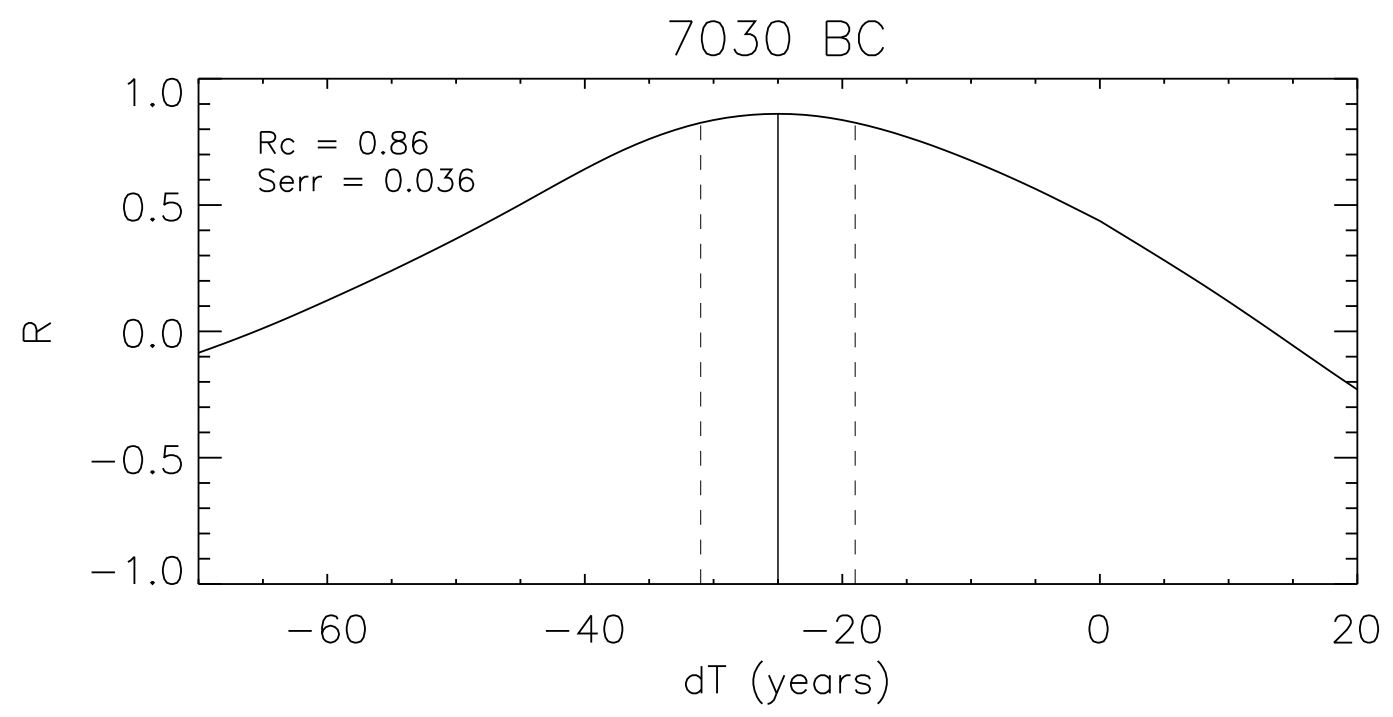

Figure 3.3: An example of the calculation of the best-fit time adjustment $d T=-25$ years (solid vertical line) and its 68\% confidence interval (dashed lines, -31 and -19 years) for the wiggle case shown in Figure 3.2. Pearson's linear correlation was calculated between ${ }^{14} \mathrm{C}$ and $\mathrm{EDML}{ }^{10} \mathrm{Be}$ series for the \pm 100 -year time window around the center of the wiggle.

vary within $+20 /-50$ years. The adjustment range for GRIP is concordant with the Greenland chronology correction function (e.g., Muscheler et al. 2014) within the uncertainties of the GICC05 timescale (Seierstad et al. 2014). Some discrepancies may be caused by the differences in the datasets and methodology applied. There are no earlier results for the EDML synchronization chronology to be compared with. We emphasize here that we do not pretend to perform a full chronological scale update but only to match wiggles between a single beryllium series and ${ }^{14} \mathrm{C}$ data, which is sufficient for this work. In particular, earlier synchronization studies produced smooth correction curves (e.g., Knudsen et al. 2009; Muscheler et al. 2014) where individual wiggles may be still slightly mismatching, while here we are focused on matching each wiggle in each series separately.

An example of the resulting re-dated ${ }^{10} \mathrm{Be}$ series compared to the reference ${ }^{14} \mathrm{C}$ record is shown with the solid black curve in Figure 3.2. The synchronization obviously improves the cross-correlation between the series as shown in Table 3.3. The improvement (in term of the ratio of $R^{2}$, which is a measure of the power of co-variability, between the original and synchronized series) is significant for the long (1.1 and 2.14 for GRIP and EDML series, respectively) and shorter Greenland series (NGRIP and Dye3) but small for short Antarctic series.

The pairwise wavelet coherence between long-term series is shown in Figure 3.4, calculated following the procedure described in Usoskin et al. (2009), including the significance estimate using the non-parametric random-phase method (Ebisuzaki 1997). One can see that the coherence between EDML ${ }^{10} \mathrm{Be}$ and ${ }^{14} \mathrm{C}$ series (panel a) is good on the timescale shorter than 1000 years and insignificant on the timescale longer than $2000-$ 3000 years, with no coherence inbetween. Coherence between GRIP ${ }^{10} \mathrm{Be}$ and ${ }^{14} \mathrm{C}$ series is good at all timescales longer than 400 - 500 years. Coherence between GRIP and 
Table 3.3: Squared correlation coefficients between the six ${ }^{10} \mathrm{Be}$ series and the ${ }^{14} \mathrm{C}$ series for the originally dated $\left(R_{\mathrm{o}}\right)$ and synchronized $\left(R_{\mathrm{s}}\right)$ series. Improvement factor $f$ is defined as the ratio of the squared correlation coefficients.

\begin{tabular}{ccccccc}
\hline \hline & GRIP & EDML & NGRIP & Dye3 & DF & SP \\
\hline$R_{\mathrm{o}}^{2}$ & 0.60 & 0.18 & 0.12 & 0.23 & 0.57 & 0.53 \\
$R_{\mathrm{s}}^{2}$ & 0.66 & 0.38 & 0.14 & 0.26 & 0.59 & 0.53 \\
$f$ & 1.11 & 2.14 & 1.11 & 1.14 & 1.03 & 1.01 \\
\hline
\end{tabular}

EDML series is intermittent on the timescales shorter than 1000 years and insignificant on the longer timescales.

\subsection{Reconstruction of the solar modulation potential}

Since cosmogenic isotopes are produced by cosmic rays in the Earth's atmosphere (Beer et al. 2012), their measured production/depositional flux reflects changes in the cosmic ray flux in the past. In turn, cosmic rays are modulated by 7 solar magnetic activity, which is often quantified in terms of the modulation potential $\phi$. The latter is a useful parameter to describe solar modulation of GCR using the so-called force-field parametrization formalism (e.g., Caballero-Lopez and Moraal 2004; Usoskin et al. 2005). In an ideal case, when both the production rate of cosmogenic isotopes and the geomagnetic field at a given time are known, the corresponding modulation parameter can be calculated for the given isotope, using a production model which considers, in great detail, all the processes of the nucleonic-muon-electromagnetic cascade triggered by energetic cosmic rays in the atmosphere. Here we use the production model by Poluianov et al. (2016), which is a recent update of the widely used Cosmic Ray Atmospheric Cascade model (CRAC; Kovaltsov and Usoskin 2010; Kovaltsov et al. 2012). This model provides absolute production rates and is in full agreement with other modern models (Pavlov et al. 2017). The modulation potential $\phi$ is defined here (see full formalism in Usoskin et al. 2005) for the local interstellar spectrum according to Burger et al. (2000). Since the modulation potential is a model-dependent parameter, our result cannot be directly compared to the $\phi$-values based on different assumptions (e.g., Steinhilber et al. 2012) without a re-calibration (Herbst et al. 2010; Asvestari et al. 2017). Thus, the $\phi$-series is an intermediate result which is further converted into an physical index of the open magnetic flux and subsequently to the sunspot numbers.

The relation between the isotope production rate and its measured content $\left(\Delta^{14} \mathrm{C}\right.$ for ${ }^{14} \mathrm{C}$ and depositional flux or concentration for ${ }^{10} \mathrm{Be}$ ) depends on the corresponding atmospheric/terrestrial cycle of the isotope. Radiocarbon is involved, as carbon-dioxide gas, in the global carbon cycle and is almost completely mixed and homogenized over the global hemisphere. Here we used the globally averaged ${ }^{14} \mathrm{C}$ production rate as computed by Roth and Joos (2013) from the INTCAL09 standard $\Delta^{14} \mathrm{C}$ dataset (Reimer et al. 2009) using a new-generation dynamic carbon cycle model including coupling with the diffusive ocean. However, the effect of extensive fossil fuel burning (Suess effect) makes it difficult to use ${ }^{14} \mathrm{C}$ data after the mid-19th century because of large and poorly constrained uncertainties (Roth and Joos 2013). Radiocarbon data cannot be used after the 1950s because of man- 

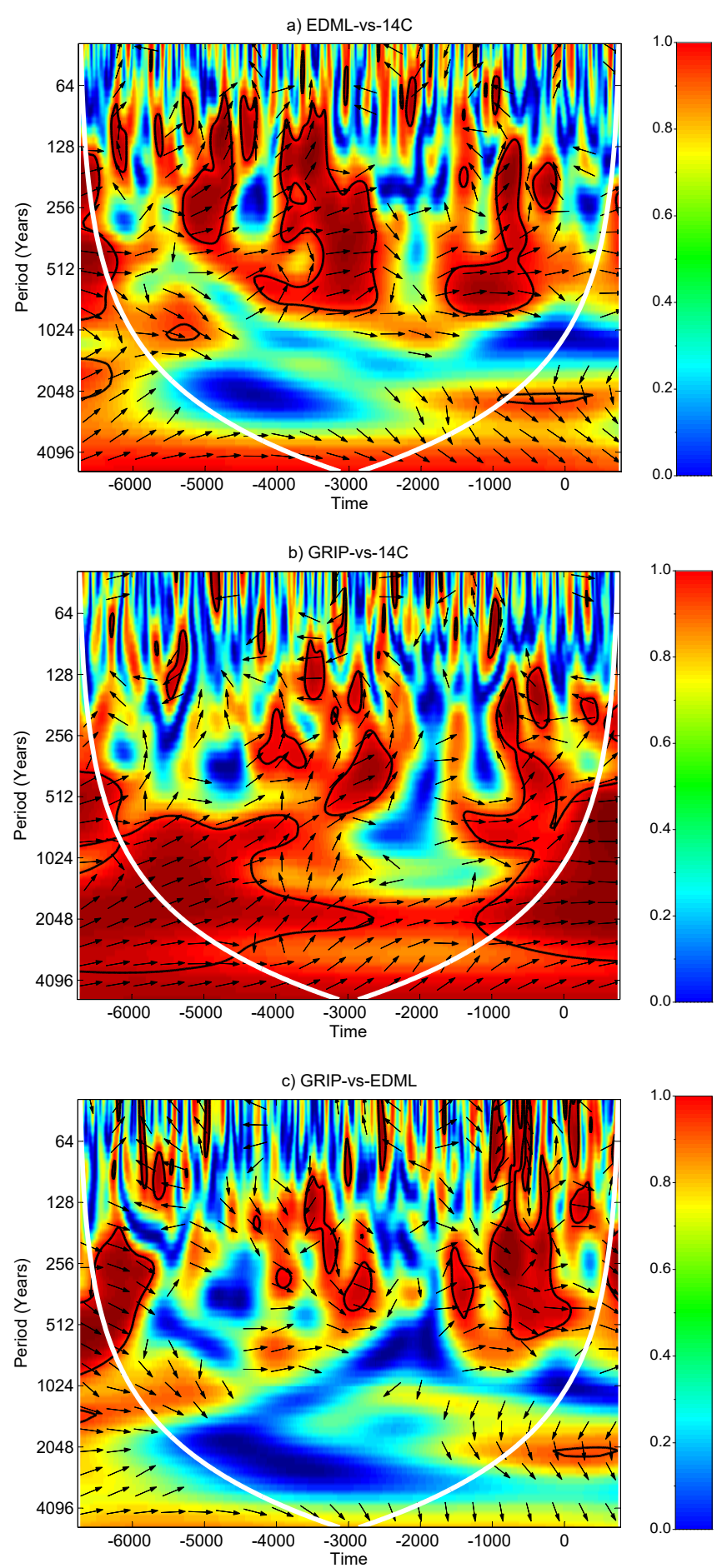

Figure 3.4: Wavelet coherence between long series, re-dated using the wiggle matching, considered here: (a) ${ }^{10} \mathrm{Be}$ EDML vs. ${ }^{14} \mathrm{C}$; (b) ${ }^{10} \mathrm{Be}$ GRIP vs. ${ }^{14} \mathrm{C}$; (c) ${ }^{10} \mathrm{Be}$ GRIP vs. ${ }^{10} \mathrm{Be}$ EDML. Color scale ranges from 0 (deep blue) to 1 (dark red). Arrows denote the relative phasing between the series: arrows pointing right denote phase matching, while pointing left - antiphase. The white curves denote the cone of influence (COI) beyond which the result is unreliable. 
made nuclear explosions leading to massive production of ${ }^{14} \mathrm{C}$. Accordingly, we do not extend our analysis towards the twentieth century (cf., e.g., Knudsen et al. 2009).

Contrary to ${ }^{14} \mathrm{C},{ }^{10} \mathrm{Be}$ is not globally mixed and its transport/deposition in the atmosphere is quite complicated and subject to local/regional conditions. Here we applied the ${ }^{10}$ Be production model by Poluianov et al. (2016) while atmospheric transport and deposition was considered via parametrization by Heikkilä et al. $(2009,2013)$ who performed a full 3-D simulation of the beryllium transport and deposition in the Earth's atmosphere. However, the existing models consider only the large-scale atmospheric transport and do not address in full detail the deposition at each specific location, that may differ significantly from site to site. This remains an unknown factor (up to 1.5 in either direction) between the modelled and actually measured deposition flux of ${ }^{10} \mathrm{Be}$ at any given location (e.g., Sukhodolov et al. 2017). On the other hand, a free conversion factor exists if the ${ }^{10} \mathrm{Be}$ data are provided in concentration units rather than depositional flux. Therefore, we considered a single scaling factor entering the production rate $Q$ to match it to the measured values, that is separately adjusted for each ${ }^{10} \mathrm{Be}$ series.

Sometimes sporadic solar energetic particle (SEP) events are produced by the Sun, with strong fluxes of energetic particles impinging on the Earth's atmosphere. Although such solar particle storms usually have a short duration and soft energy spectrum, the SEP flux can produce additional cosmogenic isotopes in the atmosphere. For extreme events, the enhancement of the isotope production may greatly exceed the annual yield from GCR (Usoskin and Kovaltsov 2012). If not properly accounted for, such events may mimic periods of reduced solar activity (McCracken and Beer 2015), since the enhanced isotope production is erroneously interpreted in terms of the enhanced GCR flux and, consequently, reduced solar activity. There are two known extreme SEP events that can lead to such an erroneous interpretation (Bazilevskaya et al. 2014): the strongest one around 775 AD (Miyake et al. 2012) and a weaker one in 994 AD (Miyake et al. 2013). Energy spectra of these events were assessed elsewhere (Usoskin et al. 2013; Mekhaldi et al. 2015). The production effect of the event on the ${ }^{10} \mathrm{Be}$ data in polar ice was calculated by Sukhodolov et al. (2017) and removed from the original data. One potential candidate around 5480 BC studied by Miyake et al. (2017) appears to be an unusual solar minimum rather than an SEP event. Accordingly, we keep it as a wiggle not correcting for the possible SEP effect.

Here we first calculated the modulation potential $\phi$ in the past for each series individually, and then for all series together. The reconstruction process is described in detail below.

\subsubsection{Reducing the series to the reference geomagnetic conditions}

Temporal variability of cosmogenic isotope production contains two signals: solar modulation and changes of the geomagnetic field, which are independent of each other, and can thus be separated. Since here we are interested in the solar variability, we removed the geomagnetic signal by reducing all production rates to the reference geomagnetic conditions, defined as follows: the geomagnetic field is dipole-like, aligned with the geographical axis, and its virtual axial dipole moment (VADM) is $8 \times 10^{22} \mathrm{~A} \mathrm{~m}^{2}$. The exact VADM value is not important for the procedure, so we have chosen a round one close to the mean value for the twentieth century. The reduction was done in two steps: 


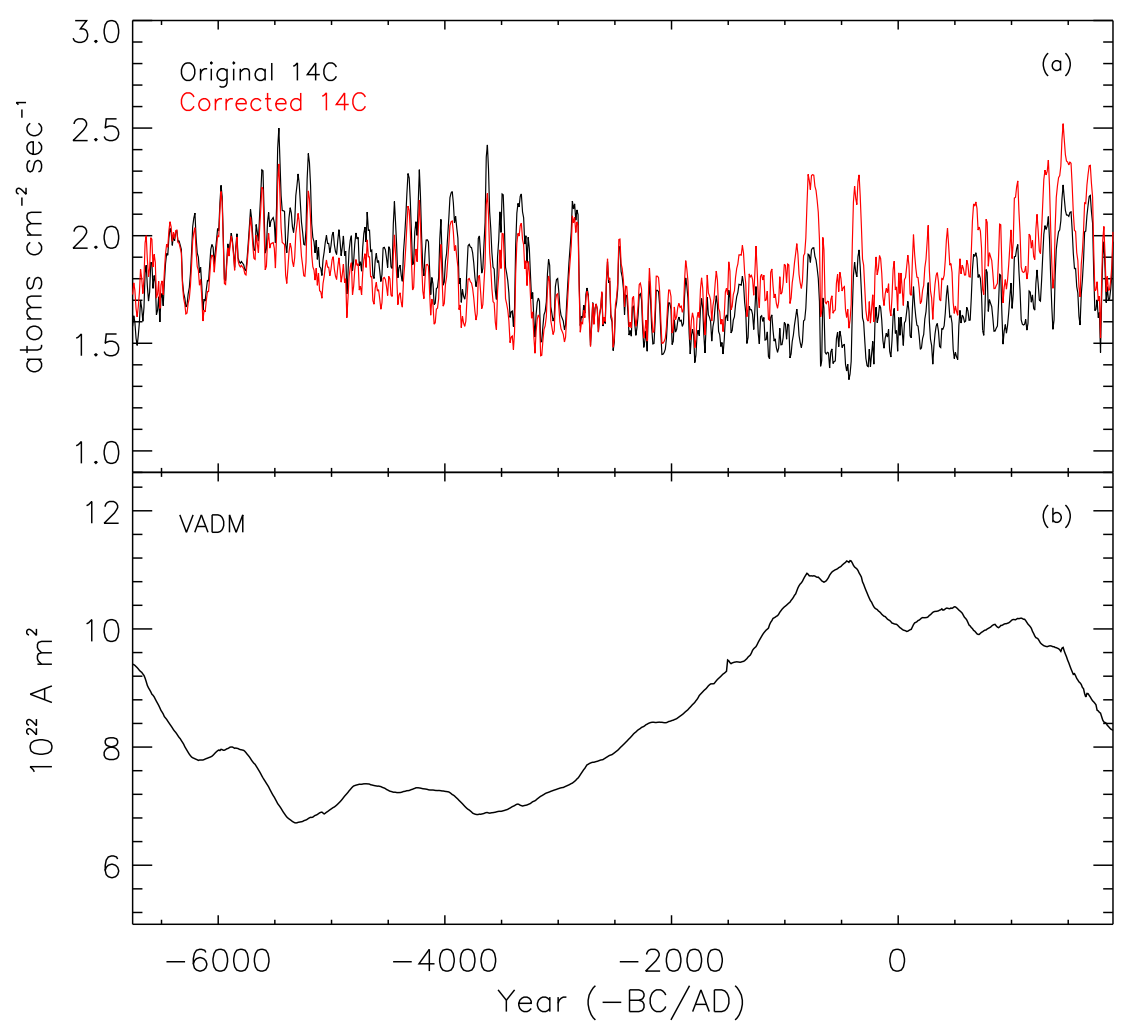

Figure 3.5: (a) Original (black) mean ${ }^{14} \mathrm{C}$ production rate (Roth and Joos 2013) and the one reduced to the standard geomagnetic conditions (red). (b) mean VADM reconstruction (Usoskin et al. 2016a).

(1) From the given isotope's production rate $Q(t)$ and the geomagnetic VADM $M(t)$ we calculated the value of $\phi(t)$ using the production model by Poluianov et al. (2016).

(2) From the value of $\phi(t)$ computed in step 1, we calculated the reduced production rate $Q^{*}(t)$ using the same production model but now fixing the VADM at $M=8 \times 10^{22}$ $\mathrm{A} \mathrm{m}^{2}$. This value roughly corresponds to the 20th century conditions. This yields the isotope production rate as it would have been during time $t$ if the geomagnetic field was kept constant at this value of $M$.

The new $Q^{*}$ series is now free (in the framework of the adopted model) of the geomagnetic changes and is used in the subsequent reconstructions. An example of the original series and that corrected for variations in the geomagnetic field is shown in Figure 3.5.

\subsubsection{Reconstruction based solely on ${ }^{14} \mathrm{C}$}

First, we calculated the solar modulation potential based solely on the radiocarbon data using the Monte-Carlo method similar to that developed by Usoskin et al. (2014, 2016a). The reconstruction includes the following steps:

(1) For each moment $t$ in time, we used 1000 realizations $Q_{i}(t)$ of the full ensemble provided by Roth and Joos (2013), which include both uncertainties of the carbon 


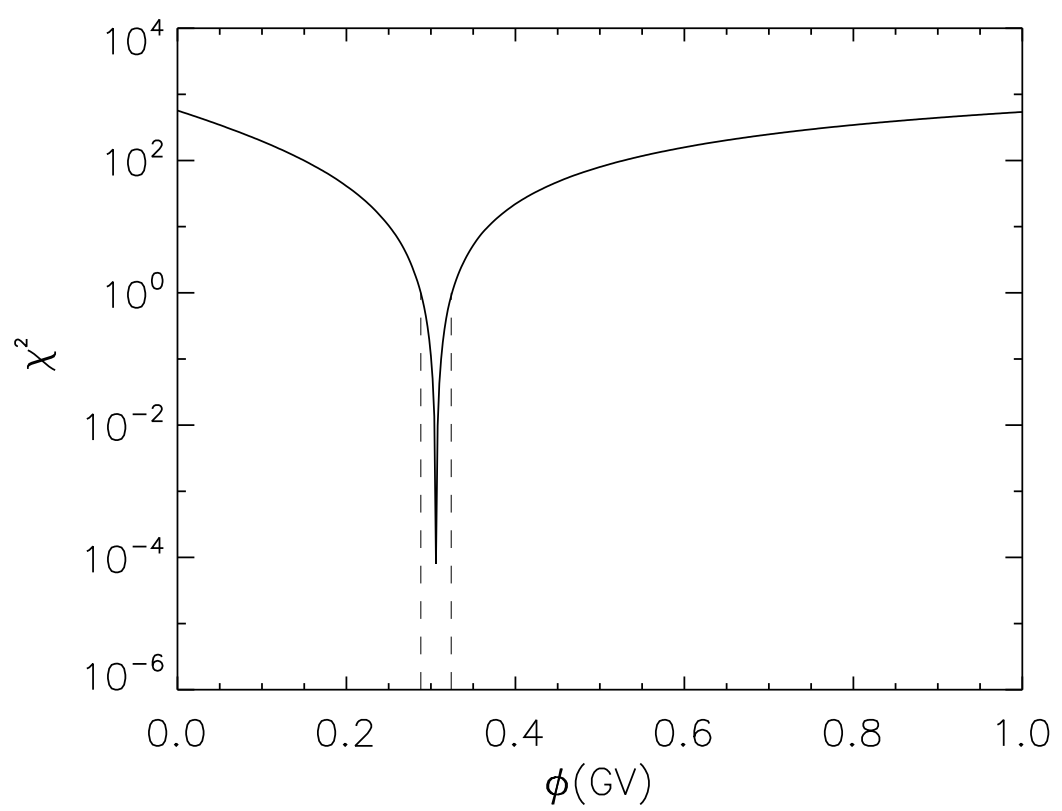

Figure 3.6: An example of the $\chi^{2}$ vs. $\phi$ dependence for the year $805 \mathrm{AD}$ for the ${ }^{14} \mathrm{C}$ series. The dashed lines represent the $68 \%$ confidence interval for $\phi$.

cycle and the measurement errors. At the same time we also used 1000 realizations of the VADM $M_{j}(t)$ from Usoskin et al. (2016a), which include uncertainties and cover the range of available archeomagnetic reconstructions to calculate $10^{6}$ values of $Q_{i j}^{*}(t)$, as described in Section 3.4.1. The whole ensemble provides a natural way to represent the range and uncertainties of the reconstructed quantities $(Q$ or VADM). For this $Q_{i j}^{*}(t)$ ensemble we calculated the mean $\left\langle Q^{*}(t)\right\rangle$ and the standard deviation $\sigma_{Q}(t)$.

(2) Using the statistics of the $Q^{*}(t)$ ensemble, we defined, for each time $t$, the best-fit $\phi(t)$ that minimizes the value of $\chi^{2}$ :

$$
\chi^{2}(\phi)=\left(\frac{\left\langle Q^{*}\right\rangle-Q^{\prime}(\phi)}{\sigma_{Q}}\right)^{2},
$$

where $Q^{\prime}(\phi)$ is the value of $Q^{*}$ computed for a given value of $\phi$, which was scanned over the range $0-2000 \mathrm{MeV}$. The best-fit value of $\phi_{0}$ is defined as that corresponding to the minimum $\chi_{0}^{2}$ ( $\rightarrow 0$ for a single series used). The $68 \%$ confidence interval of $\phi$ is defined as that bounded by the values of $\chi^{2}=\chi_{0}^{2}+1$. An example of the $\chi^{2}(\phi)$ dependence and definition of the best-fit $\phi$-values and its uncertainties is shown in Figure 3.6.

(3) The series of reconstructed $\phi$ based on ${ }^{14} \mathrm{C}\left(\phi_{14 \mathrm{C}}\right)$ was then computed, along with the uncertainties, as shown in Fig. 3.7. 


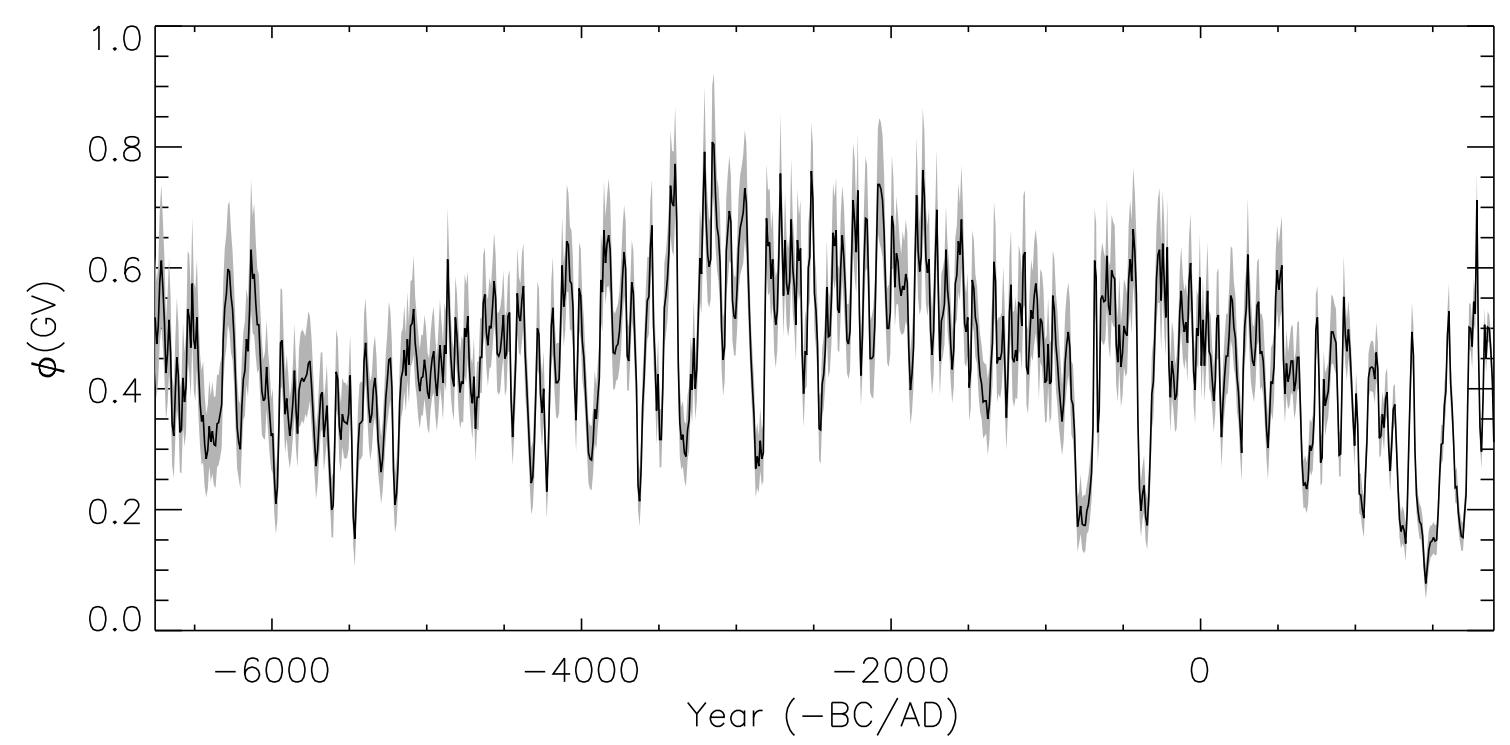

Figure 3.7: Series of the modulation potential $\phi$ computed based only on the ${ }^{14} \mathrm{C}$ data. Shading denotes the $68 \%$ confidence interval.

\subsubsection{Comparison between the long ${ }^{10} \mathrm{Be}$ and ${ }^{14} \mathrm{C}$ series}

Next we compared the long-term behaviour, in the sense of the scaling factors, of the long-running ${ }^{10} \mathrm{Be}$ series versus the reference ${ }^{14} \mathrm{C}$ series. First we considered a 1000 year window and calculated the mean value of $\left\langle\phi_{14 \mathrm{C}}\right\rangle$ within this period, as described in Section 3.4.2. We then scaled each ${ }^{10} \mathrm{Be}$ series individually with a scaling factor $\kappa$ and reconstructed the value of $\phi_{\kappa}$, for the re-scaled ${ }^{10} \mathrm{Be}$ series in this time window. The value of $\kappa$ was defined such that the mean value of $\phi_{10 \mathrm{Be}}$ agrees with that for ${ }^{14} \mathrm{C}$, for the same period, i.e. $\left\langle\phi_{10 \mathrm{Be}}\right\rangle=\left\langle\phi_{14 \mathrm{C}}\right\rangle$. Then, the 1000 -year window was moved by 100 years and the above procedure was repeated.

The resulting ${ }^{10} \mathrm{Be}$ scaling factors $\kappa$ are shown as a function of time in Figure 3.8. The $\kappa$-factor for the GRIP series is relatively stable during the period $6760 \mathrm{BC}-3000 \mathrm{BC}$ and depicts a steady monotonous decrease around $3000 \mathrm{BC}$ and reaching $-12 \%$ with respect to the final $\kappa_{G R I P}$ around $1000 \mathrm{AD}$. The Pearson's squared correlation between the two curves is significant $R^{2}=0.78$ ( $p$-value 0.02$)^{2}$ implying a possible residual effect of the geomagnetic field in the reconstruction.

It is interesting to note that the two $10 \mathrm{Be}$ series show very different trends during the second half of the Holocene. The $\kappa$-factor for the EDML series shows a weak growing trend against the ${ }^{14} \mathrm{C}$ series over the entire period of their overlap, with $\kappa$ varying from $-5 \%$ to $+10 \%$. A shallow wavy variability can be noticed, with a quasi-period of approximately 2400 years, which is probably related to Hallstatt cycle (Damon and Sonett 1991; Usoskin et al. 2016a). The correlation between the $\kappa$-factor for EDML and the VADM is insignificant $R^{2}=0.52(p=0.12)$. This suggests that most likely the under-corrected geomagnetic field effect is only related to the GRIP series.

The discrepancy between GRIP and ${ }^{14} \mathrm{C}$ series is well known (e.g., Vonmoos et al. 2006; Inceoglu et al. 2015), but has been typically ascribed to the early part of the Ho-

\footnotetext{
${ }^{2}$ The significance is estimated using the non-parametric random-phase method by Ebisuzaki (1997)
} 


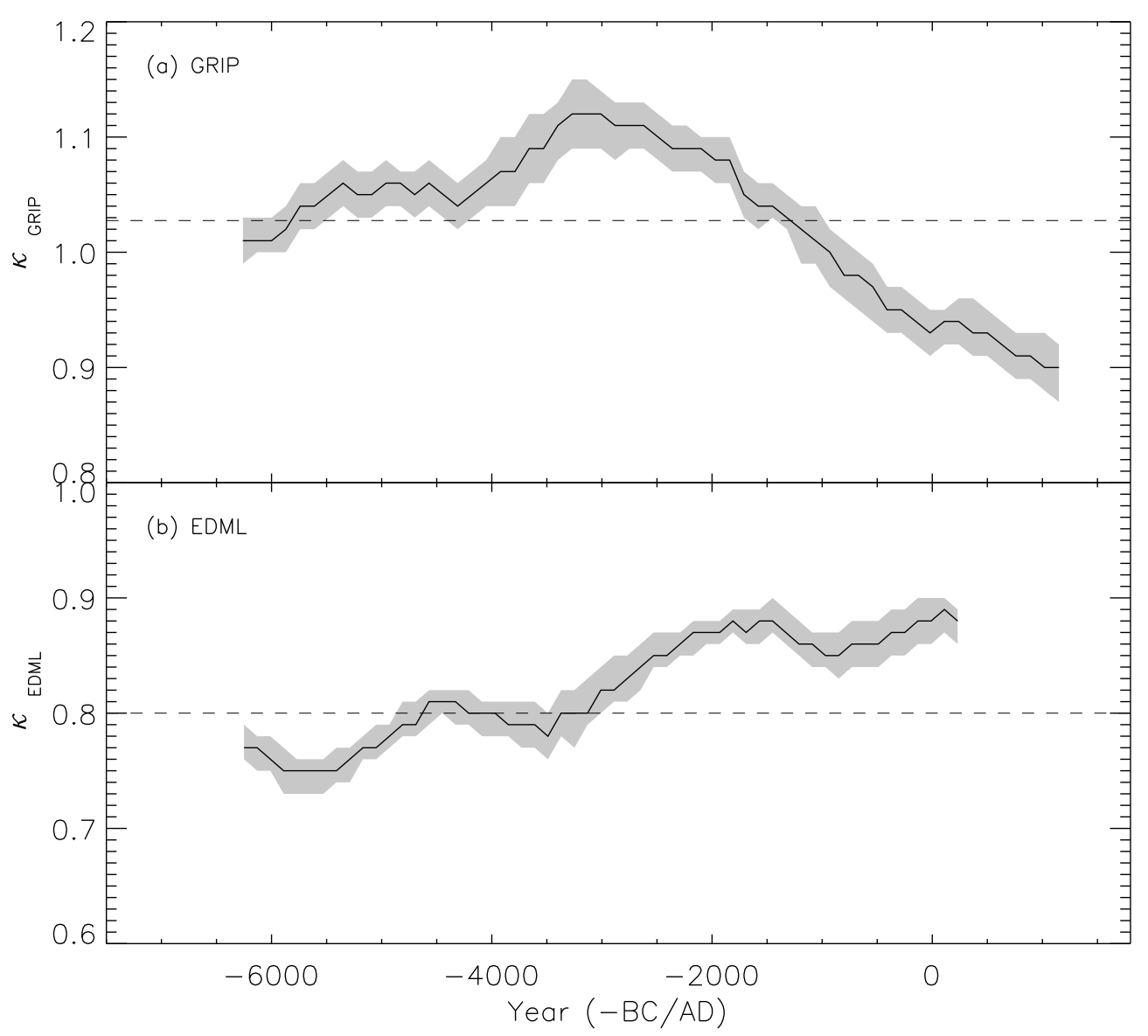

Figure 3.8: The ${ }^{10} \mathrm{Be}$ scaling factor $\kappa$, with $68 \%$ uncertainties indicated by the grey shading, in the sliding 1000-year window as a function of time. Panels a and $b$ are for the GRIP and EDML series, respectively. Dashed lines depict the $\kappa$ factor defined for the entire period (Section 3.4.5).

locene, because of the normalization of both series to the modern period. With such a normalization, the records agree with each other over the last millennia but diverge before ca. 2000 BC (Inceoglu et al. 2015). Sometimes (e.g., Usoskin 2017, and references therein) this discrepancy was also explained as a possible delayed effect (not perfectly stable thermohaline circulation) of the deglaciation in the carbon cycle (e.g., Muscheler et al. 2004). However, as we show here, this explanation is unlikely for two reasons.

More details of the long-term relation between the series are visible in Figure 3.9, where we plot the pairwise wavelet coherence between the modulation potential (shown in Figure 3.12) from the three long series. As one can see in panel a, the EDML series is coherent with the ${ }^{14} \mathrm{C}$ series at all timescales shorter than $\approx 2000$ years and again (but insignificantly) longer than 4000 years, while no coherence exists between 2000 and 4000 years. The GRIP-based series (panel $b$ ) is well coherent with the ${ }^{14} \mathrm{C}$ one on timescales longer than $100-200$ years, with the coherence disappearing at timescales longer than $2000-3000$ years. The two ${ }^{10} \mathrm{Be}$ are hardly coherent with each other on timescales longer than 1000 years (panel c), with a small insignificant isle of coherence beyond the cone of influence. It is noteworthy that each of the ${ }^{10} \mathrm{Be}$ series exhibits a higher coherence with the ${ }^{14} \mathrm{C}$ one than with each other. A similar conclusion was made by Usoskin et al. (2009) 
using different ${ }^{10} \mathrm{Be}$ data series. Uncovering a reason for this discrepancy requires further investigation.

Since the reason for this discrepancy is unknown, we do not correct it. In the following we apply fixed coefficients for ${ }^{10} \mathrm{Be}$ series, thus keeping the long-term variability as it exists in the original data.

It is interesting that while the coherence between EDML- and ${ }^{14} \mathrm{C}$-based reconstructions has been improved compared to the coherence between the raw data series (Figure 3.4), the coherence has been degraded for the GRIP series (panels b) in the long timescales above 2000 years. This suggests that beryllium may be better mixed for Greenland than yielded by the applied beryllium transport parametrization based on Heikkilä et al. (2009). The reconstruction based on the ${ }^{14} \mathrm{C}$ series has no residual correlation with VADM $\left(R^{2}<0.01\right)$. A new full-size model of the atmospheric transport and deposition of ${ }^{10} \mathrm{Be}$ in polar regions, particularly in Greenland, is needed in the future to resolve the issue.

\subsubsection{Reconstructions from individual series}

Two beryllium series, GRIP and EDML, are used here for a preliminary assessment of this method for their overlap. The mean modulation potential for the period $6800 \mathrm{BC}-$ $700 \mathrm{AD}$ based on radiocarbon is $\left\langle\phi_{14 \mathrm{C}}\right\rangle=0.468 \mathrm{GV}$.

First, we scaled each ${ }^{10} \mathrm{Be}$ series individually with a free scaling factor $\kappa_{0}$ and reconstructed the series of $\phi$. The values of $\kappa_{0}$ were defined such that the mean value $\left\langle\phi_{10 \mathrm{Be}}\right\rangle$ is equal to that for ${ }^{14} \mathrm{C}$ for the same period. The scaling factor found for the GRIP data is close to unity, $\kappa_{0}=1.1$. This implies that ${ }^{14} \mathrm{C}$ and ${ }^{10} \mathrm{Be}$ data are fully consistent in the framework of the model, and that our modelling of the cosmogenic isotope production and transport/deposition is appropriate. The best-fit scaling factor for EDML is $\kappa_{0}=0.8$, viz. about $20 \%$ lower than unity, which is reasonable considering specific features of the deposition at this site. These values of $\kappa_{0}$ are considered as initial guesses for a more precise search for the scaling factors, as described below.

\subsubsection{A combined-record reconstruction of $\phi$}

In the preceding section we calculated three series of $\phi$, from different cosmogenic isotope datasets, using their inter-calibration to the mean $\phi$ value obtained from the ${ }^{14} \mathrm{C}$ data over the overlap period. This is similar to what was done earlier (Vonmoos et al. 2006; Steinhilber et al. 2012; Usoskin et al. 2014, 2016a). Here we go further and perform a consistent Bayesian-based reconstruction of the modulation potential $\phi$. For each moment of time we find, using the measured data and knowledge of the other complementary parameters, the most probable value of $\phi$ and its uncertainty.

(1) First, we fix the scaling factors for GRIP and EDML series at their initial guess values $\kappa_{0}$ as described above.

(2) We then calculate, as described in Section 3.4.2 (step 1), $10^{6}$ realizations of the isotope production rates $Q^{*}(t)$ reduced to the standard geomagnetic conditions.

(3) The mean $\left\langle Q^{*}(t)\right\rangle$ and the standard deviation $\sigma_{Q^{*}}(t)$ are calculated over the $10^{6}$ ensemble members for each time point $t$. 

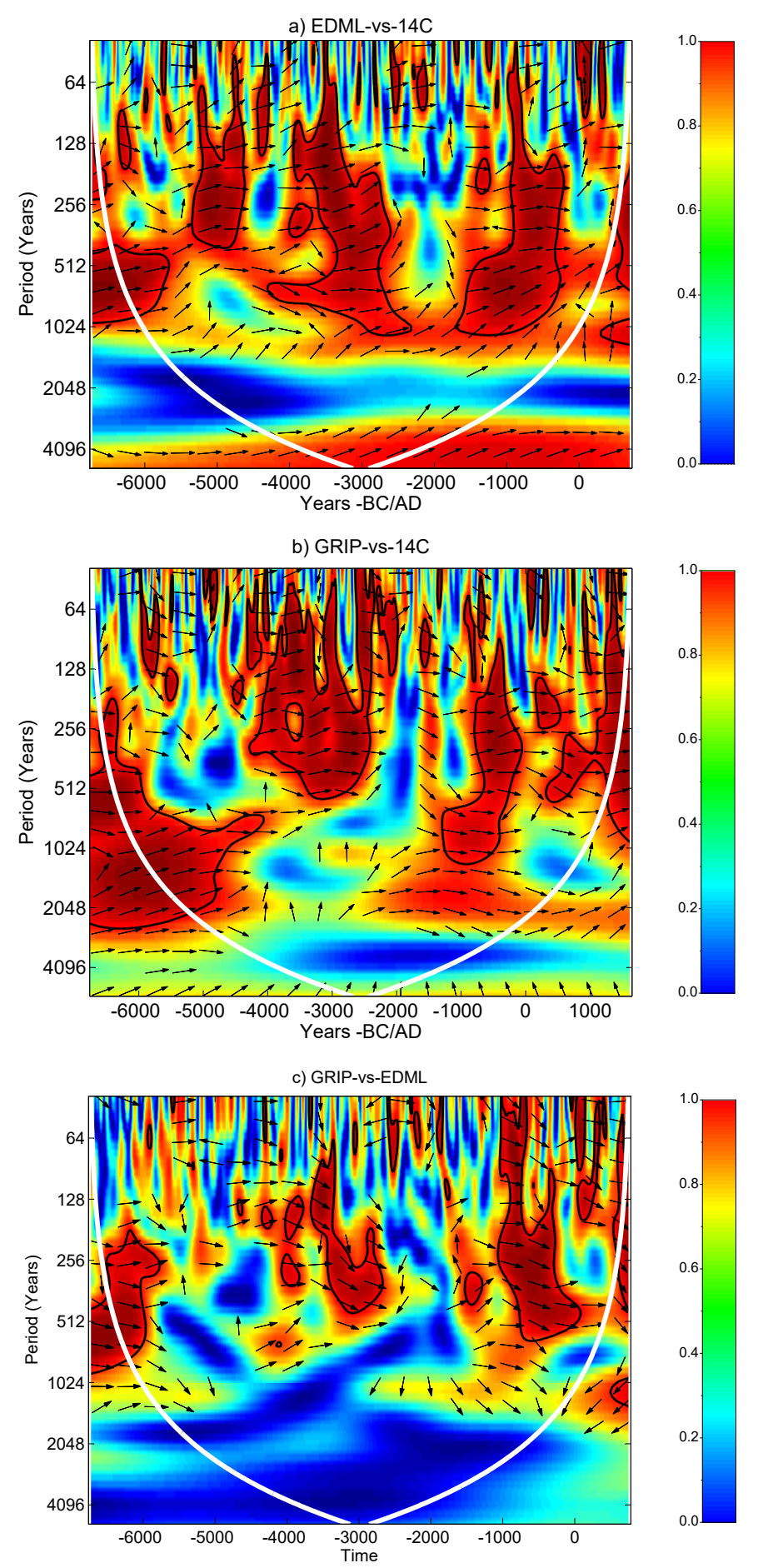

Figure 3.9: Wavelet coherence between individual long-term series of the modulation potential $\phi$ reconstructed from individual long-term cosmogenic series (shown in Figure 3.12). (a) ${ }^{10} \mathrm{Be}$ EDML vs. ${ }^{14} \mathrm{C}$; (b) ${ }^{10} \mathrm{Be}$ GRIP vs. ${ }^{14} \mathrm{C}$; (c) ${ }^{10} \mathrm{Be}$ GRIP vs. ${ }^{10} \mathrm{Be}$ EDML. Notations are similar to Figure 3.4. 
(4) For each $t$ the value of $\chi^{2}(\phi)$ is calculated as:

$$
\chi^{2}(\phi)=\sum_{i=1}^{3}\left(\frac{\left\langle Q_{i}^{*}\right\rangle-Q_{i}^{\prime}(\phi)}{\sigma_{Q_{i}}}\right)^{2},
$$

where index $i$ takes values 1 through 3 for ${ }^{14} \mathrm{C}$, GRIP and EDML series, respectively. An example of $\chi^{2}$ as a function of $\phi$ at one point in time for the three individual series as well as for their sum is shown in Figure 3.10. Each of the individual datasets (panels a-c, each similar to Figure 3.6) yields a very sharp and well defined dip in the $\chi^{2}$ value approaching zero. This is because, for a given single value of $Q$, the corresponding value of $\phi$ can be defined precisely. However, the obtained individual $\phi$-values are not identical for different datasets, leading to a smooth overall $\chi^{2}$-vs- $\phi$ dependence (panel $\mathrm{d}$ ) with the minimum $\chi^{2}$ being about 1.7 , or 0.85 per degree of freedom (DoF). This implies that the same value of $\phi=0.58 \mathrm{GV}$ satisfies all three isotope data records within statistical confidence.

(5) We calculate the sum of individual $\chi^{2}$ values (Eq. 3.3) as $\chi_{\Sigma}^{2}=\sum_{t} \chi^{2}(t)$ over all the 750 time points during $6760 \mathrm{BC}-730 \mathrm{AD}$. The corresponding sum is $\chi_{\Sigma}^{2}=4347$ or $\approx 2.9$ per DoF, indicating that there is likely a systematic difference between the series. The DoF number is defined as $750 \times 3$ (number of points in the three series) minus 752 (the number of fitted parameters), thus 1498 .

(6) Since the values of the scaling factors $\kappa_{0}$ were initially defined by normalizing the mean values of $\phi$, which is not optimal, we re-define them via $\chi^{2}$ as follows. We repeat steps (1)-(5) above, now scanning the values of $\kappa$ in the range of $0.85-$ 1.25 and $0.6-1.1$, with steps 0.01 and 0.015 , for the GRIP and EDML series, respectively. The corresponding $\chi_{\Sigma}^{2}$ are calculated. The distribution of $\chi_{\Sigma}^{2}$ as a function of $\kappa_{\mathrm{GRIP}}$ and $\kappa_{\mathrm{EDML}}$ is shown in Figure 3.11. The distribution has a clear minimum $\left(\chi_{\min }^{2}=3205\right)$ with the values of $\kappa_{\text {GRIP }}$ and $\kappa_{\mathrm{EDML}}$ being 1.028 and 0.815 , respectively, which is close to the initial guess (Section 3.4.3).

Reconstructions of $\phi$ based on the best-fit scaling for individual series are shown in Figure 3.12 as coloured curves. The differences between the data points from the different series are normally distributed with a mean value of about zero and the standard deviation of $100-200 \mathrm{MV}$. This serves as an estimate of the accuracy of individual reconstructions.

\subsubsection{Full reconstruction}

Next, we analyse all the available data series, including also four shorter ${ }^{10} \mathrm{Be}$ series, extending the $\phi$ reconstruction to 1900 AD. The four shorter series are (see Table 3.1) NGRIP and Dye3 from Greenland, as well as Dome Fuji (DF) and South Pole (SP) from Antarctica.

We first estimate the best-guess scaling $\kappa$-factors for each short series similar to what is described in Section 3.4.3, viz. equalizing the mean values of $\langle\phi\rangle$ for the series in question to that of the reference ${ }^{14} \mathrm{C}$ series for the period of their overlap. We then slightly vary the $\kappa$-value around these best-guessed values to calculate the corresponding $\chi^{2}$ for 


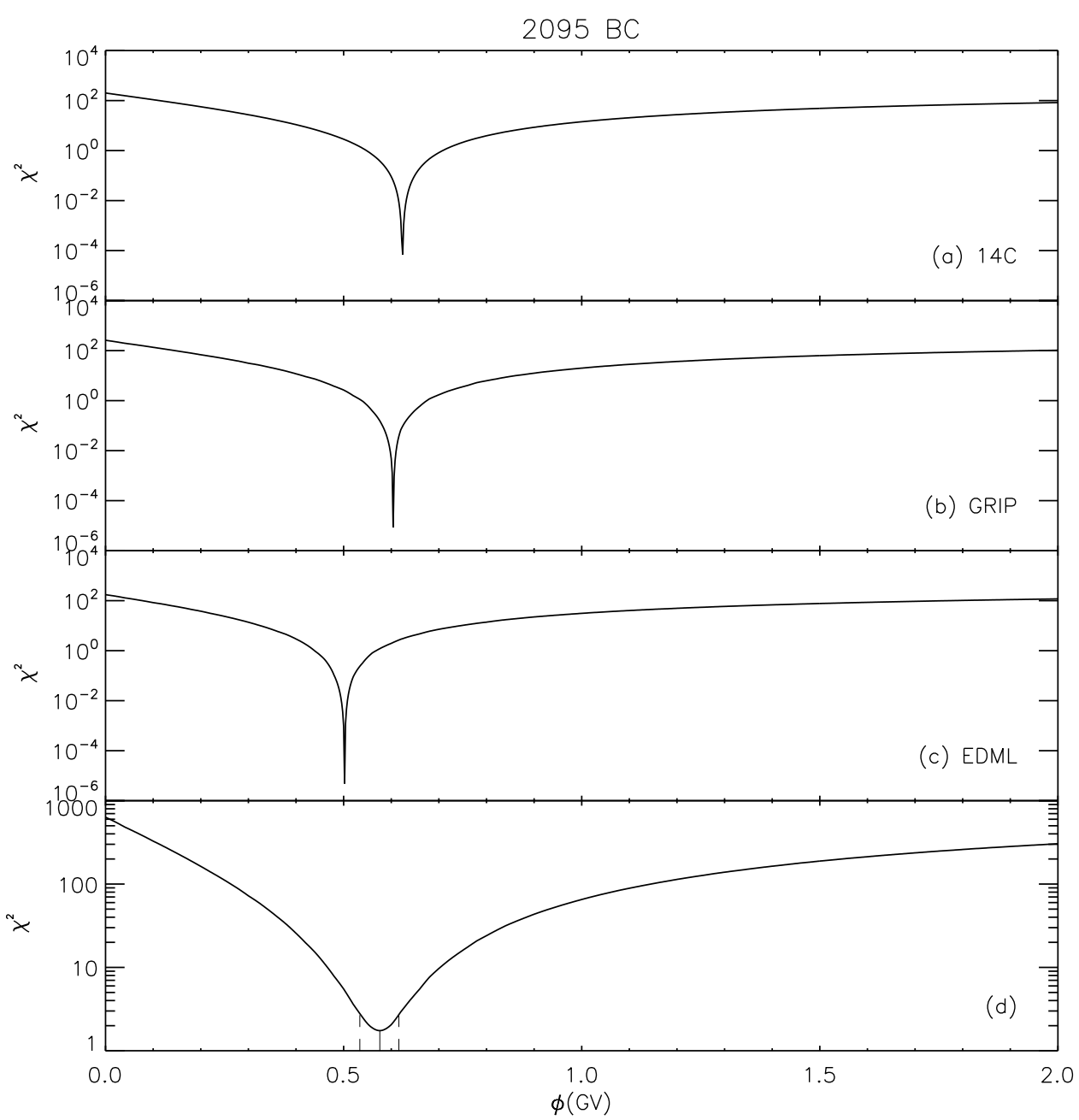

Figure 3.10: Dependence of $\chi^{2}$ (Eq. 3.3) on $\phi$ for the decade centred at 2095 BC: (a) ${ }^{14} \mathrm{C}$ with no scaling,(b) ${ }^{10} \mathrm{Be}$ GRIP with scaling $(\kappa=1.028)$, (c) EDML with scaling $\kappa=0.815$, and (d) sum of the three $\chi^{2}$ components.

each individual series, as shown in Figure 3.13. The vertical solid lines mark the bestfit $\kappa$-values which minimize the 5-point smoothed $\chi^{2}$. The best-fit scaling factors are listed in Table 3.1. The scaling factor for ${ }^{10} \mathrm{Be}$ series with depositional flux data (GRIP, EDML, NGRIP, Dome Fuji - see Table 3.1 and Figure 3.8) are close to unity (within $20 \%$ ) implying again that our model is quite realistic. The $68 \%$ uncertainties are defined as corresponding to $\left(\chi_{\min }^{2}+1\right)$.

Next, the full reconstruction using the $\chi^{2}$ method described in Section 3.4.5, but now for all the series, was performed (see Fig. 3.12). The number of different series used to reconstruct individual data points varies in time between two and five (see Figure 3.1). The mean $\chi^{2}$ per DoF is 0.57 , for $84 \%$ of data points $\chi^{2}<1$ per DoF, implying that the agreement between different series is good. 


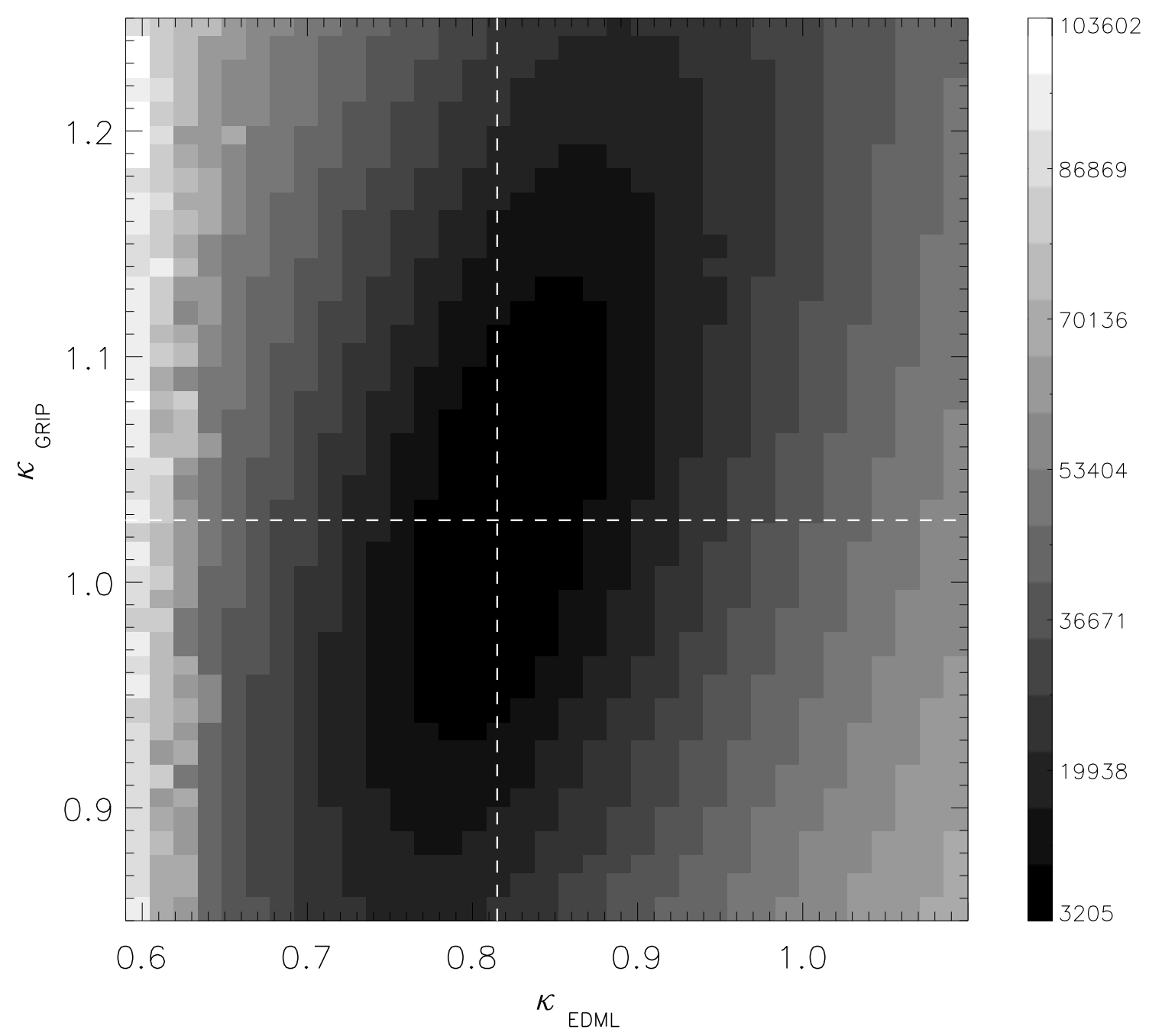

Figure 3.11: Distribution of $\chi_{\Sigma}^{2}$ versus $\kappa_{\mathrm{GRIP}}$ and $\kappa_{\mathrm{EDML}}$. Grey scale is on the right. The minimum of the distribution $\left(\chi_{\min }^{2}=3205\right)$ occurs at $\kappa_{\mathrm{GRIP}}=1.028$ and $\kappa_{\mathrm{EDML}}=0.815$, as marked by the cross-hairs.

\subsection{Reconstruction of sunspot number}

The modulation potential series alone are not very useful as a solar activity proxy since the modulation potential is a relative index whose absolute value is model dependent (Usoskin et al. 2005; Herbst et al. 2010, 2017). Therefore, we convert the modulation potential, reconstructed in Section 3.4 into a more definitive index, the sunspot number $S N$. This is done via the open solar magnetic flux $F_{\mathrm{o}}$, following an established procedure (e.g., Usoskin et al. 2003; Solanki et al. 2004; Usoskin et al. 2016a). Applying the updated SATIRE-M model (Vieira and Solanki 2010; Vieira et al. 2011; Wu et al. 2018a), we can write the relation (Usoskin et al. 2007, see the Appendix therein) between the two indices as

$$
\mathrm{SN}_{i}=116 \times \phi_{i}+33 \times\left(\phi_{i+1}-\phi_{i}\right)-16
$$

where the sunspot number during the $i$-th decade, $\mathrm{SN}_{i}$, is defined by the modulation potential (expressed in GV) during the contemporary and the following decades. The negative 

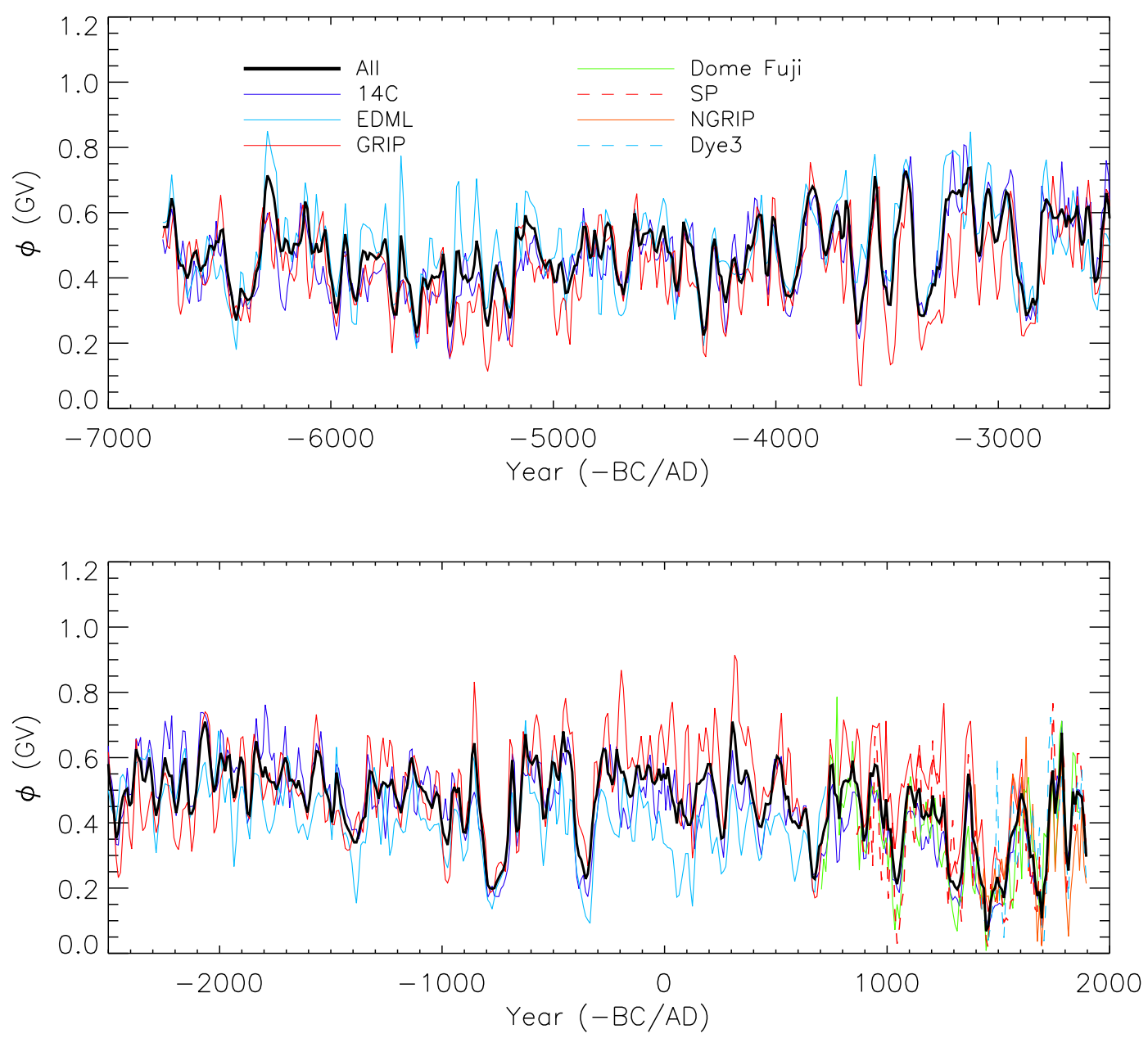

Figure 3.12: Reconstruction of the modulation potential $\phi$ using only individual cosmogenic isotope series (color curves as denoted in the legend) with the best-fit scaling (see Table 3.1) and the final composite series (thick black curve). Top and bottom panels depict the two halves of the entire interval. Only mean values are shown without uncertainties.

offset term (-16) reflects the fact that zero sunspot number during Grand minima does not imply the absence of GCR modulation, so that even when $\mathrm{SN}=0$ the value of $\phi$ is about 0.14 GV (e.g., Owens et al. 2012). Since sunspot numbers cannot be negative, the SN was assigned zero values when the values of $\phi$ drop below the sunspot formation threshold. The uncertainties of the reconstructed SN-values were defined by converting the low- and upper-bound (68\%) $\phi$-values (defined as described above) for each decade into sunspot numbers. The reconstructed sunspot number series ${ }^{3}$ is shown in Figure 3.14 along with its $68 \%$ confidence interval and compared with the International sunspot number series (SILSO ISN version 2, Clette et al. 2014) ${ }^{4}$. Since we use sunspot numbers in their 'classical' definition, the ISN v.2 data were scaled down with the factor 0.6 (as described in

\footnotetext{
${ }^{3}$ Available as a table in the Supplement materials and at the MPS Sun-Climate web-page http://www . mps.mpg.de/projects/sun-climate/data

${ }^{4}$ File SN_y_tot_V2.0.txt available at http: //www.sidc.be/silso/infosnytot
} 

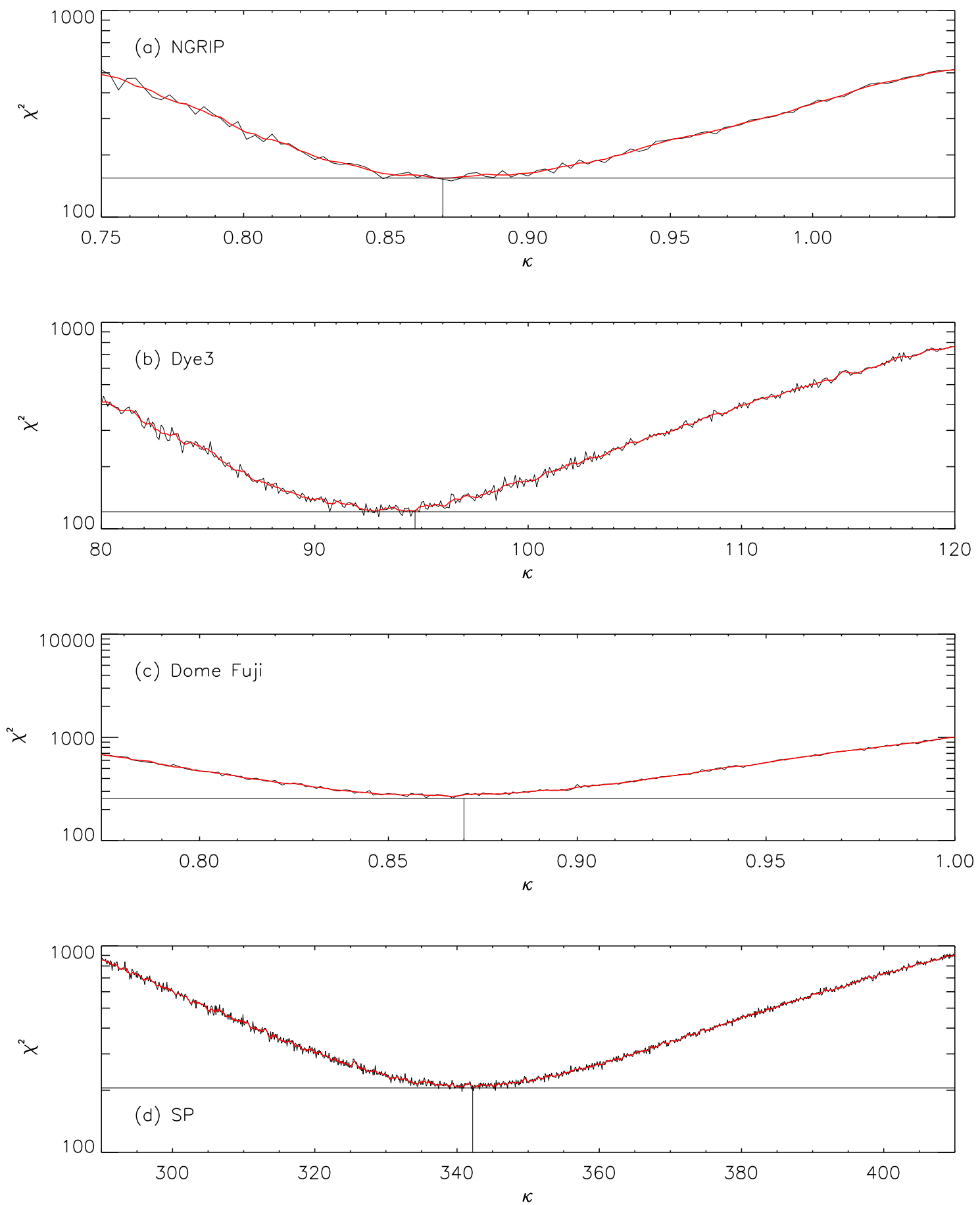

Figure 3.13: Values of $\chi^{2}$ versus the scaling factors $\kappa$ for four short ${ }^{10}$ Be series, as marked in each panel. The 5-point running mean curves are shown in red. The locations of the best-fit $\kappa$-values are shown by the straight black lines. 


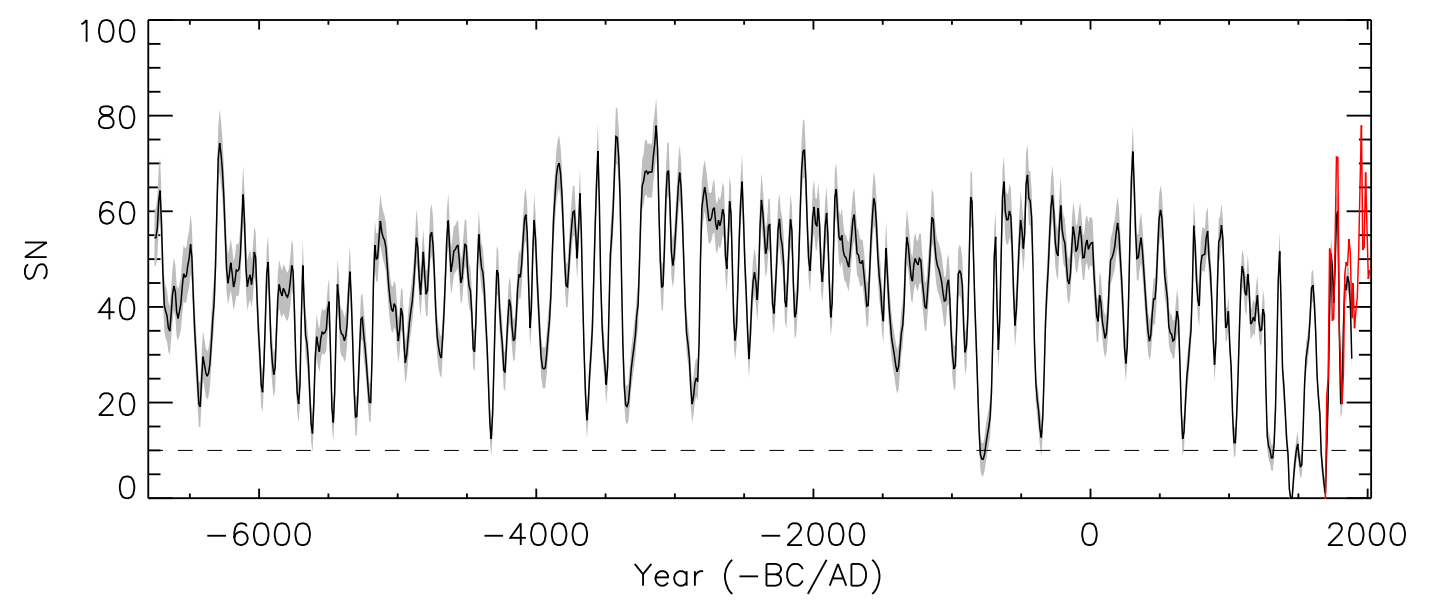

Figure 3.14: Reconstructed sunspot number along with its $68 \%$ confidence interval (gray shading). This series is available in the Support Data. Red line depicts the decadally resampled International sunspot number (version 2, scaled by 0.6) from Clette et al. (2014). The dashed line denotes the level of $\mathrm{SN}=10$.

Clette et al. 2014).

One can see that the reconstructed solar activity varies at different time scales, from decades to millennia. In particular, there is a long period with relatively high activity between roughly $4000 \mathrm{BC}$ and $1500 \mathrm{BC}$, and periods of lower activity ca. $5500 \mathrm{BC}$ and $1500 \mathrm{AD}$. The origin of this is unclear. For example, Usoskin et al. (2016a) suggested, based on the fact that the GRIP and ${ }^{14} \mathrm{C}$ series behave differently at this time scale, that it is a global climate effect rather than solar. As a result, they removed this wave from the data in an ad-hoc manner. However, as we show here, it is more likely related to the under-correction of the GRIP-based series. Therefore, it may be related to solar activity so that we retain it in the final dataset.

The new series is generally consistent with previous reconstructions (e.g., Usoskin et al. 2016a) but it also has some new features. In particular, it implies a lower activity during the sixth millennium BC. We note that a possible overestimate of solar activity for that period was suspected earlier (Usoskin et al. 2007, 2016a).

Although the overall level of solar activity may vary significantly, the periods of Grand minima may correspond to a special state of the solar dynamo (Schmitt et al. 1996; Küker et al. 1999; Moss et al. 2008; Choudhuri and Karak 2012; Käpylä et al. 2016) and thus are expected to provide roughly the same low level of activity, corresponding to a virtual absence of sunspots. Thus, the level of the reconstructed activity during clearly distinguishable Grand minima may serve as a rough estimate of the 'stability' of the reconstruction, if we assume that activity always drops to the same, nearly zero level during each Grand minimum (Sokoloff and Nesme-Ribes 1994; Usoskin et al. 2014). The expected level of solar activity during Grand minima $(\mathrm{SN} \rightarrow 0)$ is indicated by the horizontal dashed line in Figure 3.14. One can see that the level of the reconstructed Grand minima (observed as sharp dips) is roughly consistent with $\mathrm{SN} \rightarrow 0$ throughout almost the entire period, considering the uncertainty of the order of 10 in $\mathrm{SN}$ units. However, periods of $3500 \mathrm{BC}-$ 
$2500 \mathrm{BC}$ and before $5500 \mathrm{BC}$ are characterized by a slightly higher SN level during the Grand minima suggesting for a possible overestimate of activity during those times. Interestingly, there were no clear Grand minima during $2500 \mathrm{BC}-1000 \mathrm{BC}$, suggesting that it was a long period of stable operation of the solar activity main mode.

The overall level of solar activity remained roughly constant, around $45-50$, during most of the time, but appeared somewhat lower (around 40) before $5000 \mathrm{BC}$, suggesting for a possible slow variability with a timescale of about $6-7$ millennia (cf., Usoskin et al. 2016a). The reason for that is not known, but this could be due to: (1) climate influence, although this would be expected to affect ${ }^{14} \mathrm{C}$ and ${ }^{10} \mathrm{Be}$ isotopes differently; (2) solar activity, or (3) large systematic uncertainty in the geomagnetic field reconstruction which is poorly known before about $3000 \mathrm{BC}$.

Figure 3.15 shows the kernel density estimation of the probability density function (KDF) of the reconstructed decadal sunspot numbers for the entire period (877 decades). One can see the high peak of the distribution with values around 40 which corresponds to the moderate activity. Quite clear is also a low activity component with values below 15 , corresponding to Grand minima. In addition, there is a hint for a bump at the high activity tail (SN greater than 60, visible only as a small excess above the main Gaussian curve) suggesting a Grand maximum component. In order to illustrate this, we applied a formal multi-peak (Gaussian) fit to the KDF as shown by the blue dotted curves. The main peak is centred at decadal $\mathrm{SN}=42(\sigma=10)$ and represents the main component. Another peak can be found as a small bump around $\mathrm{SN}=27(\sigma=6)$, but it is not sufficiently separated from the main component to justify calling it a separate component. The Grand minimum component (Gaussian with $\sigma=6$ centred at $\mathrm{SN}=12$ ) is significantly separated from the main component (statistical significance $p<0.05$ ). The separation of the Grand maximum component, while visible by eye as a deviation from the Gaussian shape at high values, is not statistically significant. The statistical separation of the special Grand minimum component was first shown by Usoskin et al. (2014) for the last three millennia, while the result for the Grand maximum component was inconclusive.

Here we fully confirm this result over nine millennia, which implies that Grand minimum and normal activity components form a robust feature of solar variability. This also suggests that the new reconstruction is more robust and less noisy than previous ones (e.g., Steinhilber et al. 2012; Usoskin et al. 2016a) and allows to statistically identify the Grand minimum component over 9000 years.

\subsection{Conclusions}

We have provided a new fully consistent multi-proxy reconstruction of solar activity over about nine millennia, based for the first time on a Bayesian approach. We used all the available datasets of cosmogenic radioisotopes with sufficient length and quality in terrestrial archives and up-to-date models of isotope production and transport/deposition as well as a recent archeomagnetic model. We used six ${ }^{10} \mathrm{Be}$ series of different lengths from both Greenland and Antarctica, as well as the official INTCAL global ${ }^{14} \mathrm{C}$ series. Earlier reconstructions were based on either individual datasets or on a statistical superposition of those (e.g., Steinhilber et al. 2012). Our new method is based on finding the most probable value of the solar modulation potential which matches all the data for a given point in 


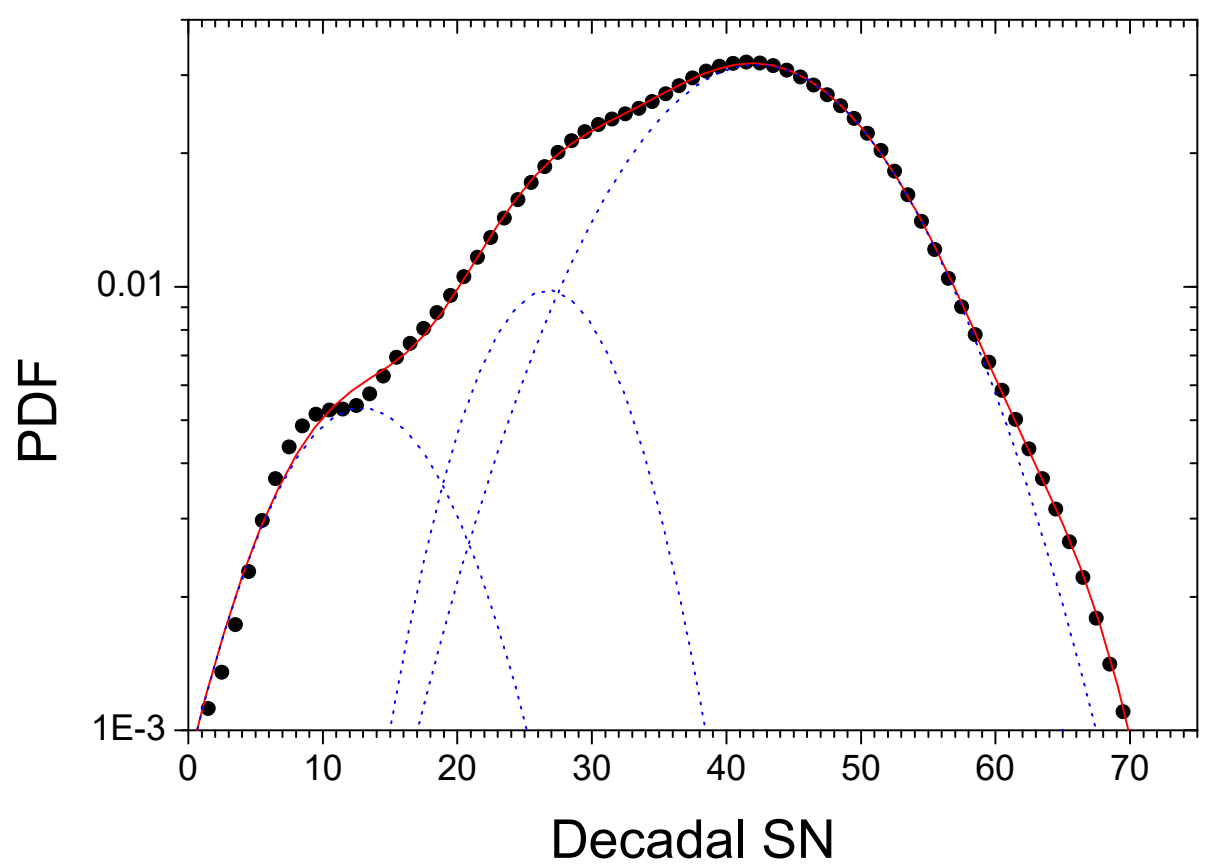

Figure 3.15: Probability kernel density function estimate (black dots) of the reconstructed decadal sunspot numbers (Gaussian kernel, width=3) and its multi-peak fit (red).

time, providing also a straightforward estimate of the uncertainties. Prior to the analysis, long ${ }^{10} \mathrm{Be}$ series were formally re-dated to match wiggles in the ${ }^{14} \mathrm{C}$ data. All employed cosmogenic isotope series were reduced to the reference geomagnetic field conditions. We have also tested the stability of the two long ${ }^{10} \mathrm{Be}$ series and found that they appear to diverge from each other during the second half of the Holocene, while the ${ }^{14} \mathrm{C}$ series lies in between them. The GRIP-based series appears to anti-correlate with the long-term geomagnetic VADM series, implying a possible under-correction for the geomagnetic shielding effect for GRIP location, while EDML and ${ }^{14} \mathrm{C}$-based reconstructions do not show any significant residual correlation with VADM. This suggests that the applied model of beryllium transport and deposition does not work properly for the Greenland site but is reasonably good for the Antarctic site. A full-size transport/deposition model (e.g., Sukhodolov et al. 2017) needs to be applied in the future to resolve this issue.

The reconstructed series (Figure 3.14) shows variability on different time scales. Of particular interest are Grand minima of activity, visible as strong dips in the time series with the level of decadal sunspot numbers below $10-15$. Another feature of the long-term evolution of solar activity is a slow variability on the $6-7$ millennia timescale with lows ca. $5500 \mathrm{BC}$ and $1500 \mathrm{AD}$. Such behaviour was interpreted by Usoskin et al. (2016a) as a possible effect of climate influence on the carbon cycle, but the fact that the ${ }^{14} \mathrm{C}$ series lies between the two diverging ${ }^{10} \mathrm{Be}$ series on this time scale makes this explanation unlikely. The cause of the feature remains unknown.

Most of the time solar activity varies slightly around the moderate level of $\mathrm{SN} \approx 40$, corresponding to the main component of solar activity. Yet, Grand minima form a statistically distinguishable component. The existence of this component was first found for the last three millennia by Usoskin et al. (2014), who interpreted it as special mode of the solar dynamo. Its confirmation here for nine millennia implies that it is a robust feature of 
solar activity. At the same time, a possible existence of a component representing Grand maxima is hinted (cf. Usoskin et al. 2014) although it cannot be separated from the main component in a statistically significant manner. We speculate that the different components of the activity distribution may be related to different modes of the operation of the dynamo.

Finally, a new consistent reconstruction of solar activity (in the form of decadal sunspot numbers) is presented that offers a more reliable estimate of the long-term evolution of solar variability and poses robust constraints on the development of solar/stellar dynamo models as well as solar-terrestrial studies. 



\section{Solar total and spectral irradiance reconstruction over the last 9000 years}

*This chapter has been submitted as a journal paper to A\&A by C.-J $\mathrm{Wu}^{1,2}$, N. A. Krivova ${ }^{1}$, S. K. Solanki ${ }^{1,3}$ and I. G. Usoskin ${ }^{4,5}$.

\section{Abstract}

Contents. Changes in solar irradiance and in its spectral distribution are among the main natural drivers of Earth's climate. However, irradiance measurements are only available for less than four decades, while assessment of solar influence on Earth requires much longer records.

Aims. The aim of this work is to provide the most up-to-date physics-based reconstruction of the solar total and spectral irradiance (TSI/SSI) over the last nine millennia.

Methods. The concentrations of the cosmogenic isotopes ${ }^{14} \mathrm{C}$ and ${ }^{10} \mathrm{Be}$ in natural archives have been converted to the decadally-averaged sunspot numbers through a chain of physics-based models. TSI and SSI are reconstructed with an updated SATIRE model. Reconstructions are carried out for each isotope record separately, as well as for their composite.

Results. We present the first ever SSI reconstruction over the last 9000 years from the individual ${ }^{14} \mathrm{C}$ and ${ }^{10} \mathrm{Be}$ records as well as from their newest composite. The reconstruction employs physics-based models to describe the involved processes at each step of the procedure.

Conclusions. Irradiance reconstructions based on two different cosmogenic isotope records, ${ }^{14} \mathrm{C}$ and ${ }^{10} \mathrm{Be}$, agree well with each other in their long-term trends despite their different geochemical paths in the Earth atmosphere. Over the last 9000 years, the range of the secular changes in TSI are of the order of $0.11 \%$, or $1.5 \mathrm{~W} / \mathrm{m}^{2}$. After the Maunder minimum, the reconstruction from the cosmogenic isotopes is consistent with that from the direct sunspot number observation. Furthermore, over the 18th to 19th century, the

\footnotetext{
${ }^{1}$ Max-Planck-Institut für Sonnensystemforschung, Justus-von-Liebig-Weg 3, Göttingen, Germany

${ }^{2}$ Georg-August-Universität Göttingen, Institute for Astrophysics, Göttingen, Germany

${ }^{3}$ School of Space Research, Kyung Hee University, Yongin, Gyeonggi-Do,446-701, Republic of Korea

${ }^{4}$ Space Climate Research Unit, University of Oulu, Finland

${ }^{5}$ Sodankylä Geophysical Observatory, University of Oulu, Finland
} 
agreement with the reconstruction from the sunspot number by Chatzistergos et al. (2017) is better than with that from the WDC-SILSO sunspot number series (Clette et al. 2014).

\subsection{Introduction}

The Sun is the dominant external energy source to Earth's system (Kren et al. 2017) and is thus expected to affect its climate (Hansen 2000; Haigh 2001, 2003; Gray et al. 2010; Solanki et al. 2013). Although solar variability and its influence on Earth have been studied for a long time by various approaches, the physical processes and the level of solar influence on Earth's coupled atmosphere-ocean system are not yet fully understood. Variability in solar irradiance is considered among the possible mechanisms. The total solar irradiance (TSI) is the spectrally-integrated energy flux per unit area normalised to $1 \mathrm{AU}$, while the solar spectral irradiance (SSI) is the flux per unit wavelength. TSI (which is dominated by the radiation in the visible and infrared, IR, bands of the spectrum) and SSI (in particular, in the ultraviolet, UV, spectral range) are the main agents of the socalled bottom-up and top-down mechanisms, respectively (Gray et al. 2010). The bottomup mechanism is induced by the solar irradiance absorbed by Earth's surface while the top-down one is associated with the interaction between the ultraviolet irradiance and the stratosphere (Haigh 1994, 2001). As UV radiation below $400 \mathrm{~nm}$ most probably contributes more than a half of the TSI variability (Krivova et al. 2006; Ermolli et al. 2013; Yeo et al. 2014b, 2017a; Morrill et al. 2014; Woods et al. 2015), SSI and the UV range are of particular interest to studies of solar influence on climate.

To better understand the mechanisms of solar impact on climate, long-term records of solar irradiance are required. Both TSI and SSI are measured since 1978 by space-based instruments, yet for longer time scale suitable models are needed. A number of models have been published (Lean 2000; Krivova et al. 2007, 2010; Shapiro et al. 2010; DasiEspuig et al. 2014, 2016; Coddington et al. 2016) that reconstruct solar total and spectral irradiance on decadal to centennial time scales from various solar activity proxies, such as the Ca II K index, solar radio flux $f 10.7 \mathrm{~cm}$, sunspot areas, or the sunspot number. Also isotope concentrations in terrestrial archives have been utilised to evaluate TSI on the millennial time scale (Steinhilber et al. 2009, 2012; Delaygue and Bard 2011; Shapiro et al. 2011; Vieira et al. 2011). Of these, only Shapiro et al. (2011) have also considered SSI, while only Vieira et al. (2011) have used physics-based models to describe the whole chain of the involved processes at each step. Other models have relied on the linear regressions between the measured irradiance and the input proxies, while Vieira et al. (2011) have demonstrated that the linear relationship is actually not applicable. Here we follow the approach of Vieira et al. (2011) to update their TSI reconstruction and to also, for the first time, reconstruct SSI from the UV to the far-IR, considering physical process instead of relying on linear relationships. Also, for the first time, we present a reconstruction based on a composite ${ }^{10} \mathrm{Be}$ and ${ }^{14} \mathrm{C}$ records.

We use the SATIRE model (Spectral and Total Irradiance REconstruction, Fligge et al. 2000; Krivova et al. 2003, 2007, 2010; Wenzler et al. 2004, 2006; Vieira et al. 2011; Ball et al. 2014; Yeo et al. 2014b; Dasi-Espuig et al. 2014, 2016), which is a family of models based on the assumption that the irradiance variation on time scales longer than about one day is driven solely by the changes in the photospheric magnetic field. This model 
family attributes the irradiance changes to the competing contributions of dark (sunspot and pores) and bright (faculae and the network) surface magnetic features, as all other variable irradiance models do. The brightness of individual components is assumed to be time-independent, but their surface coverage changes with time. The positions and the areas of the various surface magnetic components are most accurately extracted from solar full-disc magnetograms, as used in the SATIRE-S model ("S" stands for satellite era, Fligge et al. 2000; Krivova et al. 2003; Wenzler et al. 2006; Ball et al. 2014; Yeo et al. 2014 b), but these are only available (with a suitable quality and cadence) since 1974. In the period 1610 - 1974, SATIRE relies on sunspot observations and the corresponding version is termed SATIRE-T (where "T" stands for telescope era, Balmaceda et al. 2007; Krivova et al. 2007, 2010), and SATIRE-T2 for the version using the surface flux transport model to restore the evolution of the solar surface magnetic flux (Dasi-Espuig et al. 2014, 2016). The sunspot number has not been recorded prior to 1610 but can be reconstructed from the cosmogenic isotope concentrations, ${ }^{14} \mathrm{C}$ and ${ }^{10} \mathrm{Be}$ retrieved from tree rings and ice cores, respectively (Beer 2000a; Solanki et al. 2004; Usoskin et al. 2007; Steinhilber et al. 2009, 2012; Vieira et al. 2011; Usoskin 2017).

The production rate of cosmogenic isotopes depends on the intensity of galactic cosmic rays (GCRs) that enter Earth's atmosphere. GCRs have to penetrate the heliosphere and the Earth's magnetic field before interacting with nuclei of atmospheric atoms and producing radionuclides that are later stored in terrestrial archives. Therefore, the abundance of isotopes in archives depends not only on the heliospheric magnetic field, but also on the geomagnetic field (shielding effect, Vonmoos et al. 2006). As the GCR flux impinging on Earth is modulated by the solar open magnetic flux (Masarik and Beer 1999; Usoskin et al. 2002; Caballero-Lopez and Moraal 2004), the concentration of cosmogenic isotopes is an indirect indicator of solar activity. A distinct inverse proportional relationship between GCR flux and solar activity has been observed (Usoskin 2017).

Although one can reconstruct the sunspot number from cosmogenic isotope records, these data have a much lower temporal resolution than the daily observed sunspot number: only decadal-averaged values are available on the millennial timescale. Therefore, the SATIRE-T model cannot be applied directly. It has been adapted to deal with this complication by Vieira and Solanki (2010) and Vieira et al. (2011), and the corresponding version of the model is termed SATIRE-M ("M" for millennia). Vieira and Solanki (2010) have shown that the relationship between the irradiance and quantities that are usually derived from the cosmogenic data is non-linear, and Vieira et al. (2011) have used this model to reconstruct the TSI, for the first time avoiding a linear relationship. They used the solar open magnetic flux (OMF) derived by Usoskin et al. (2007) from the isotope ${ }^{14} \mathrm{C}$ measurements to reconstruct the solar surface magnetic fields over the Holocene for two geomagnetic models. Recently, Usoskin et al. (2016a) have reconstructed sunspot number back to $6755 \mathrm{BC}$ from an updated geomagnetic model and two cosmogenic isotope series, ${ }^{14} \mathrm{C}$ (Roth and Joos 2013) and ${ }^{10} \mathrm{Be}$ (Yiou et al. 1997; Muscheler et al. 2004; Vonmoos et al. 2006). Furthermore, Wu et al. (2018b) have combined one global ${ }^{14} \mathrm{C}$ and six local ${ }^{10} \mathrm{Be}$ series into a single state-of-the-art multi-isotope composite record. They also used it to reconstruct the sunspot number over the Holocene.

Here we reconstruct the TSI and SSI using the updated model of Vieira et al. (2011) and the most up-to-date sunspot reconstructions by Usoskin et al. (2016a) and Wu et. al (2018). We also extend the model to reconstruct both the total and the spectral irradiance. 
The concept and the methods of the SATIRE model are introduced in Section 4.2. The cosmogenic isotope data are described in Section 4.2.3.1, and the reconstruction of solar irradiance is presented in Section 4.3. The results and conclusions are summarized in Section 4.4.

\subsection{Model description}

\subsubsection{The general concept of the SATIRE model}

In the SATIRE models, the solar surface is described by five components: umbra, penumbra, facula, network and the quiet Sun. The features evolve with time and move across the visible disc as the Sun rotates. Thus the positions of and the fractional disc coverage by the features change. On each given day this information is taken from solar observations. In particular, when daily high-resolution full-disc solar magnetograms and continuum images are available for the last 4 decades, the SATIRE-S model is able to replicated the directly measured irradiance variability accurately. However, one has to rely on less complete sets of observations for going further back in time .

The longest record of direct solar observations is the sunspot number that extends back to the Maunder minimum, although with progressively degrading quality (see Sect. 4.2.2.1). SATIRE-T employs the sunspot numbers to first reconstruct the evolution of the solar photospheric magnetic fields through a set of ordinary differential equations (Solanki et al. 2000, 2002b; Vieira and Solanki 2010), which is then used as input to the reconstruction of the solar irradiance (Balmaceda et al. 2007; Krivova et al. 2007, 2010). Before 1610 only indirect data are available, and thus Vieira and Solanki (2010) and Vieira et al. (2011) have adapted the SATIRE model to use the radioisotope data as input (SATIRE-M). This requires an additional step of first reconstructing the solar activity (either the sunspot number or the solar OMF) from the concentrations of ${ }^{14} \mathrm{C}$ or ${ }^{10}$ Be records (e.g., Solanki et al. 2004; Steinhilber et al. 2012; Usoskin et al. 2014). In this study, to reconstruct the solar total and spectral irradiance on both centennial and millennial time scales, we will use the SATIRE-T model for the period from the Maunder minimum to the present and the SATIRE-M model over the period 6755 BC to 1895 AD. More details of the SATIRE-T and SATIRE-M model are given in Sect. 4.2.2 and Sect. 4.2.3, respectively.

In all versions of the model, the input data are used to recover the fractional solar disc coverage by a given photospheric component at a given time, which is described by the corresponding filling factor $\alpha$ (note that the definition of the filling factors is somewhat different in the version of the model that uses spatially-resolved solar magnetograms and images, but this is irrelevant for this work). The brightness of each component $i$ is timeindependent and is calculated following Unruh et al. (1999) using the ATLAS9 spectral synthesis code (Kurucz 1993) from the corresponding solar model atmospheres (for details see Unruh et al. 1999). Intensity spectra $I_{i}(\lambda, \mu)(\mu=\cos \theta$, where $\theta$ is the heliospheric angle) of the quiet Sun, umbra and penumbra are calculated from the model atmospheres of Kurucz $(1993,2005)$ with effective temperatures of 5777 K, $4500 \mathrm{~K}$ and $5400 \mathrm{~K}$, respectively. The model atmosphere used for faculae and network was adapted from the model $\mathrm{P}$ by Fontenla et al. (1999). The intensity spectra, $I_{i}(\lambda, \mu)$, are then integrated over 
the whole disc (or over the activity belts, see Sect. 4.2.2.2) to give the disc-integrated brightness spectra, $F_{i}(\lambda)$, of each component.

The solar irradiance at a given wavelength, $\lambda$ (SSI), at a given time, $t$, is obtained by summing up spectra of the individual photospheric components weighted by their surface coverage, given by their filling factors $\alpha_{i}$ :

$$
F(\lambda, t)=\sum_{i=\mathrm{u}, \mathrm{p}, \mathrm{f}, \mathrm{n}, \mathrm{q}} \alpha_{i}(t) F_{i}(\lambda)
$$

The ATLAS9 spectral synthesis code uses the assumption of Local Thermodynamic Equilibrium (LTE), which breaks down in the solar chromosphere where the important parts of solar UV radiation are formed. Krivova et al. (2006) and Yeo et al. (2014b) have shown that the SATIRE spectra need a correction below $300 \mathrm{~nm}$. Following Yeo et al. (2014b), between 180 and $300 \mathrm{~nm}$ we introduce an offset in the absolute values to match the Whole Hemispheric Interval (WHI) reference solar spectrum (Woods et al. 2009), while in the range $115-180 \mathrm{~nm}$, the variability is rescaled with empirical factors derived using the SORCE/SOLSTICE measurements (Snow et al. 2005). Note that these corrections do not affect the long-term trend. Finally, TSI is calculated as an integral of SSI over the entire spectral range $115-160000 \mathrm{~nm}$.

\subsubsection{SATIRE-T}

\subsubsection{Surface magnetic field}

In the SATIRE-T model, the evolution of the solar surface magnetic field is described by a set of coupled ordinary differential equations (ODEs). The model has been described in detail in earlier papers (Solanki et al. 2002b; Krivova et al. 2007; Vieira and Solanki 2010). Therefore, here we only briefly outline its main features. Three components of the magnetic field are considered: the magnetic field in active regions, $\varphi_{\text {act }}$, ephemeral regions, $\varphi_{\mathrm{eph}}$, and the open flux, $\varphi_{\mathrm{open}}$. Active regions (ARs) include sunspots and faculae. They typically emerge in the so-called activity belts (i.e., within roughly latitudes of 5 $-30^{\circ}$ for sunspots and $5-45^{\circ}$ for faculae) and show a pronounced solar activity cycle. Ephemeral regions (ERs) are small-scale bipolar regions with much shorter lifetimes distributed more homogeneously over the solar surface. They show a much weaker, if any, variation over the solar cycle (see Harvey 1993; Hagenaar 2001; Hagenaar et al. 2003). The emergence of ARs and ERs in our model is described by the corresponding emergence rates, $\varepsilon_{\text {act }}$ and $\varepsilon_{\text {eph }}$, respectively. This magnetic field decays on time scales of $\tau_{\text {act }}^{0}$ and $\tau_{\mathrm{eph}}^{0}$. Part of the field is trapped by the solar wind plasma and dragged further into the heliosphere, forming the so-called open flux. Whereas part of the open flux decays within a few months, some part of it can survive much longer (about $3-4$ years, Solanki et al. 2000, 2002b; Vieira and Solanki 2010; Owens and Lockwood 2012). Thus the slowly and rapidly decaying components of the open flux, $\varphi_{\mathrm{open}}^{\mathrm{s}}$ and $\varphi_{\mathrm{open}}^{\mathrm{r}}$, respectively, are modelled separately, with the corresponding decay times being $\tau_{\text {open }}^{\mathrm{s}}$ and $\tau_{\text {open }}^{\mathrm{r}}$. The flux transfer from ARs and ERs to the slowly evolving open flux occurs on time scales $\tau_{\text {act }}^{\mathrm{s}}$ and $\tau_{\mathrm{eph}}^{\mathrm{s}}$, while $\tau_{\text {act }}^{\mathrm{r}}$ is the time scale for the transfer of the AR flux to the rapidly evolving open flux. The evolution of the magnetic field is then described by the following equations:

$$
\frac{d \varphi_{\mathrm{act}}}{d t}=\varepsilon_{\mathrm{act}}-\frac{\varphi_{\mathrm{act}}}{\tau_{\mathrm{act}}^{0}}-\frac{\varphi_{\mathrm{act}}}{\tau_{\mathrm{act}}^{\mathrm{s}}}-\frac{\varphi_{\mathrm{act}}}{\tau_{\mathrm{act}}^{\mathrm{r}}},
$$




$$
\begin{gathered}
\frac{d \varphi_{\mathrm{eph}}}{d t}=\varepsilon_{\mathrm{eph}}-\frac{\varphi_{\mathrm{eph}}}{\tau_{\mathrm{eph}}^{0}}-\frac{\varphi_{\mathrm{eph}}}{\tau_{\mathrm{eph}}^{\mathrm{s}}}, \\
\frac{d \varphi_{\mathrm{open}}^{\mathrm{r}}}{d t}=\frac{\varphi_{\mathrm{act}}}{\tau_{\mathrm{act}}^{\mathrm{r}}}-\frac{\varphi_{\mathrm{open}}^{\mathrm{r}}}{\tau_{\mathrm{open}}^{\mathrm{r}}}, \\
\frac{d \varphi_{\mathrm{open}}^{\mathrm{s}}}{d t}=\frac{\varphi_{\mathrm{act}}}{\tau_{\mathrm{act}}^{\mathrm{s}}}+\frac{\varphi_{\mathrm{eph}}}{\tau_{\mathrm{eph}}^{\mathrm{s}}}-\frac{\varphi_{\mathrm{open}}^{\mathrm{s}}}{\tau_{\mathrm{open}}^{\mathrm{s}}} .
\end{gathered}
$$

As ARs typically include sunspots, the sunspot number can be considered as a good indicator of the emergence of the magnetic field in ARs. Balmaceda et al. (2007), Vieira and Solanki (2010) and Krivova et al. (2010) used the group sunspot number (GSN) by Hoyt and Schatten (1998) as input, while Krivova et al. (2007) considered both the group and the International (or Zürich) number. The group and the International sunspot numbers agree over the last 150 years but show different overall activity levels between 1700 and 1850. A recalibration and multiple corrections to sunspot observations is the subject of an intense ongoing debate (Clette et al. 2014; Lockwood et al. 2014c; Usoskin et al. 2016c; Cliver and Ling 2016; Chatzistergos et al. 2017; Svalgaard et al. 2017), which remains a controversial topic. The effect of the choice of the input sunspot number on the irradiance reconstructions has been considered by Kopp et al. (2016) and is beyond the scope of this study. Briefly, the choice of the input series affects the overall irradiance level in the 18th and early 19th centuries but not the magnitude of the secular change between the Maunder minimum (1637 - 1715) and now (Krivova et al. 2007; Kopp et al. 2016). Furthermore, as the main goal of this work is a reconstruction of the solar irradiance over a much longer period using the cosmogenic isotope data, the level of the solar activity in the 18th century will at the end be derived independently of the sunspot data.

Thus we here use the updated International (Zürich) sunspot number series (SN version $2.0, R_{\mathrm{i}}$ hereafter) by WDC-SILSO ${ }^{1}$ as our input sunspot number. However, this SN series only extends back to 1700 which is the end phase of the Maunder minimum. Furthermore, this data set is problematic during the early 18th century due to the large amount of missing sunspot number data and the difficulty of calibrating between different observatories. Vaquero et al. (2015) have accounted for this issue and revised the sunspot number during this period. We therefore replace the sunspot number between $1639-1715$ with the one reconstructed by Vaquero et al. (2015). The original $R_{\mathrm{i}}$ series is given at three cadences, covering different periods: daily (1818 - present), monthly (1749 - present) and yearly (1700 - present). In this study, we interpolate the monthly sunspot number from 1749 - 1818 and yearly averaged before 1749 to a daily cadence for consistency in the input temporal resolution for the SATIRE-T model.

Following Solanki et al. (2002b); Krivova et al. (2007, 2010) and Vieira and Solanki (2010) we assume the emergence rate of the AR magnetic flux, $\varepsilon_{\text {act }}$, to be directly related to $R_{\mathrm{i}}$ :

$$
\varepsilon_{\mathrm{act}}=\varepsilon^{\max , 21} \frac{R_{\mathrm{i}}}{R_{\mathrm{i}}^{\max , 21}}
$$

where $\varepsilon^{\max , 21}$ and $R_{\mathrm{i}}^{\max , 21}$ are the emergence rate and the $\mathrm{SN}$ value observed during the maximum of cycle 21, obtained from a 3-month moving average (Schrijver and Harvey 1994; Vieira and Solanki 2010).

\footnotetext{
${ }^{1}$ http://sidc.oma.be/silso/datafiles
} 
We further assume that the emergence rate of ERs is linked to that of the active regions. This is based on the studies of the ERs evolution by Harvey and Martin (1973) and Harvey (1992, 1993). In particular, Wilson et al. (1988) and Harvey (1992) found that ERs start emerging earlier than ARs belonging to the same activity cycles. Thus the ER cycles last longer than the corresponding sunspot cycles and overlap during solar minima. Following Vieira and Solanki (2010), we define the ER emergence rate $\left(\varepsilon_{\mathrm{eph}}\right)$ as follows:

$$
\varepsilon_{\mathrm{eph}}(t)=\sum_{n=1} \varepsilon_{\mathrm{act}}^{\max , n} X g^{n}
$$

where $X$ is the amplitude factor and $g^{n}$ defines the ER cycle shape:

$$
g_{n}(t)= \begin{cases}\cos ^{2}\left(\frac{\pi\left(t-t_{n}^{\mathrm{max}}\right)}{L_{n}^{\mathrm{phh}}}\right), & -\frac{L_{n}^{\mathrm{act}}}{2}-L_{n}^{\mathrm{ext}} \leq t-t_{n}^{\mathrm{max}} \leq \frac{L_{n}^{\mathrm{act}}}{2}+L_{n}^{\mathrm{ext}} \\ 0, & \text { otherwise. }\end{cases}
$$

Here $t_{n}^{\max }$ stands for the time when cycle $n$ reaches its maximum and $L_{n}^{\text {act }}$ is the corresponding cycle length. The function $g^{n}$ ensures that the ER cycle maxima are at or before the corresponding sunspot cycle maxima (see Dasi-Espuig et al. 2014). The ER cycle length of the corresponding activity cycle is defined as:

$$
L_{n}^{\mathrm{eph}}=L_{n}^{\mathrm{act}}+2 L_{n}^{\mathrm{ext}}
$$

where $2 L_{n}^{\text {ext }}$ is the time extension related to each activity cycle with a constant extension parameter $c_{\mathrm{X}}$ :

$$
L_{n}^{\mathrm{ext}}=L_{n}^{\mathrm{act}}-c_{\mathrm{x}} .
$$

The decay and transfer times for the surface magnetic components are free parameters in the SATIRE-T model, which are obtained from a comparison of the results with various observations, details are described in Sect. 4.2.2.3.

\subsubsection{Filling factors}

The calculated magnetic flux for each component as a function of time is converted to filling factors entering Eq. (4.1) as follows. First, the fractional disc area coverage by sunspots is obtained directly from the measured sunspot areas whenever these are available (Balmaceda et al. 2005, 2009; Yeo et al. 2017a) ${ }^{2}$.

For the period prior to Greenwich observations (i.e., before 1874) when the area record is not available, we extrapolate it using sunspot numbers. Sunspots are divided into umbra and penumbra using their observed mean area ratio: $\alpha_{\mathrm{u}} /\left(\alpha_{\mathrm{u}}+\alpha_{\mathrm{p}}\right)=\alpha_{\mathrm{u}} / \alpha_{\mathrm{s}}=0.2$ (see, e.g., Solanki 2003). Assuming the mean field strength in penumbra is $550 \mathrm{G}$ and in umbra 1800 $\mathrm{G}$ (Keppens and Martínez Pillet 1996), we obtain the total magnetic flux in sunspots. The magnetic flux in faculae is then the difference between the total flux in ARs and that in sunspots, $\varphi_{\mathrm{f}}=\varphi_{\mathrm{act}}-\varphi_{\mathrm{u}}-\varphi_{\mathrm{p}}$. The magnetic flux in the network is the sum of the ERs and open flux: $\varphi_{\mathrm{n}}=\varphi_{\mathrm{eph}}+\varphi_{\text {open }}$.

To convert the magnetic flux in faculae $\varphi_{\mathrm{f}}$, and in network $\varphi_{\mathrm{n}}$ into filling factors $\alpha_{\mathrm{f}, \mathrm{n}}$, we assume the filling factors to vary linearly with the corresponding magnetic flux until

\footnotetext{
${ }^{2}$ http://www2.mps.mpg.de/projects/sun-climate/data/sunspot_area_psi_20170531. txt
} 
4 Solar total and spectral irradiance reconstruction over the last 9000 years

Table 4.1: Parameters and their allowed ranges in SATIRE-T model

\begin{tabular}{lcrrrrr}
\hline \hline Parameter $^{a}$ & Notation & \multicolumn{2}{c}{ Range } & \multicolumn{2}{c}{ Kea10 } & This work \\
\hline ER decay time & $\tau_{\text {eph }}^{0}$ & & & 0.0016 (fixed) \\
AR decay time & $\tau_{\text {act }}^{0}$ & & & 0.3 & 0.3 (fixed) \\
Slow OF decay time & $\tau_{\text {open }}^{\mathrm{s}}$ & 0.0016 & 4.0 & 2.97 & 3.75 \\
Rapid OF decay time & $\tau_{\text {open }}^{\mathrm{r}}$ & 0.08 & 0.36 & 0.16 & 0.14 \\
AR to slow OF transfer time & $\tau_{\text {act }}^{\mathrm{s}}$ & 10 & 90 & 71.2 & 88.3 \\
AR to rapid OF transfer time & $\tau_{\text {act }}^{\mathrm{r}}$ & 0.0016 & 3.0 & 2.1 & 2.6 \\
ER to slow OF transfer time & $\tau_{\text {eph }}^{\mathrm{s}}$ & 10 & 90 & 17.8 & 20.6 \\
\hline Saturation flux in faculae & $B_{\text {sat,f }}$ & 50 & 850 & 156 & 371 \\
Saturation flux in network & $B_{\text {sat,n }}$ & & & 800 (fixed) & \\
\hline ER cycle amplitude factor & $X$ & 70 & 150 & 78 & 106 \\
ER cycle extension & $c_{\mathrm{x}}$ & 5 & 8 & 5.01 & 7.63 \\
Fraction of detectable ER flux & $c_{\mathrm{e}}$ & & & 0.3 (fixed) & 0.4 (fixed) \\
\hline
\end{tabular}

Notes. ${ }^{(a)}$ Time is in unit [year] and magnetic flux in unit [G].

References. Kea10: Krivova et al. (2010)

the saturation flux is reached. The filling factors are 1 above the saturation limit (Fligge et al. 2000; Krivova et al. 2003, 2007; Yeo et al. 2014b). The saturation flux in the network $\left(B_{\mathrm{sat}, \mathrm{n}}\right)$ is fixed at $800 \mathrm{G}$ (for details, see Krivova et al. 2007), while the saturation limit in faculae $\left(B_{\text {sat,f }}\right)$ is a free parameter in the model (Krivova et al. 2003)

The derived facular and network magnetic fluxes are disc-integrated, and so this approach assumes that they are homogeneously distributed on the solar surface. This assumption might overestimate the contribution of faculae to the total solar irradiance since in reality, AR faculae may emerge within the so-called activity belts (at latitudes $\pm 5-45^{\circ}$ for faculae and $\pm 5-30^{\circ}$ for sunspots), while the brightness of faculae increases toward the limb (Ortiz et al. 2002; Yeo et al. 2013). Krivova et al. (2007) used simple weighting factors to account for this. In this study, instead of integrating the intensity spectra, $I_{i}(\lambda, \mu)$ of sunspot and active regions over the whole disc, we integrate them over the activity belts.

\subsubsection{Model optimisation}

As mentioned in Sect. 4.2.2.1, some of the decay and transfer times in the Eqs. (4.2) - (4.5) are free parameters in the SATIRE-T model. There are in total 12 parameters in the SATIRE-T model, as summarised in Table 4.1, of which 4 parameters can be inferred from observations whereas others are rather uncertain and are therefore considered to be free. The ER decay time $\left(\tau_{\mathrm{eph}}^{0}\right)$ is taken to be 0.0016 years following Hagenaar (2001), the AR decay time $\left(\tau_{\text {act }}^{0}\right)$ is set to be 0.3 years (Krivova et al. 2010) and the saturation field in the network $\left(B_{\text {sat, } n}\right)$ is fixed at $800 \mathrm{G}$ (Krivova et al. 2007). One more parameter, the fraction of the detectable ER flux, $c_{\mathrm{e}}$, which is described later in this section is also fixed to 0.4 . The other 8 parameters are left free within the ranges based on observations and physical constrains (for details, see Krivova et al. 2007, 2010; Vieira and Solanki 2010). These 8 free parameters are fixed from a comparison of the model outcomes with various 
available observations, as described in the following.

The observations used to compare with and to fix the free parameters are: (1) PMOD TSI composite since 1978 (Fröhlich 2006, 2009) ${ }^{3}$; (2) ground-based total magnetic flux (TMF) measurements by Wilcox Solar Observatory (WSO), National Solar Observatory(NSO) and Mount Wilson Observatory (MWO) covering cycles 21 - 23 (Arge et al. 2002; Wang et al. 2005); (3) OMF over the period 1845 - 2010 reconstructed by Lockwood et al. (2014a) from the $a a$-index; (4) 200 - $400 \mathrm{~nm}$ SSI UV reconstructed from magnetograms with the SATIRE-S model over the period 1976 - 2015 (Yeo et al. 2014b, 2015 ) and from the $110.7 \mathrm{~cm}$ radio flux with the EMPIRE model over 1947 - 1976 (Yeo et al. 2017a); and (5) facular contribution to the TSI variation calculated with SATIRE-S over 1974 - 2015 (Yeo et al. 2014b, 2015).

All data sets have been updated since the previous reconstructions by Krivova et al. (2007, 2010). A major update concerns the OMF. The updated series spans 60 years longer than the previous version by Lockwood et al. (2009) that covered only from 1904 to 2010. Note that being the longest observational series, the OMF record mostly affects the long-term trend.

When comparing the reconstructed TMF to the measured one, we take into account the finding by Krivova and Solanki (2004) that more than half of the ER flux is not detected in the magnetogram archives used here due to the insufficient spatial resolution of the instruments or is hidden in the noise. Therefore, when comparing to the observations, we reduce the amount of the ER flux by a factor $c_{\mathrm{e}}$, i.e, the TMF is computed as $\varphi_{\text {tot }}=$ $\varphi_{\text {act }}+c_{\mathrm{e}} \varphi_{\mathrm{eph}}+\varphi_{\mathrm{open}}$, where $c_{\mathrm{e}}$ describes the fraction of detectable ER flux. We follow previous studies and take $c_{\mathrm{e}}=0.4$ (Balmaceda et al. 2007; Krivova et al. 2007; DasiEspuig et al. 2016). This factor is not used when calculating the irradiance, as in this case it is the total amount of the solar surface magnetic flux which is of relevance.

To optimise and constrain the free parameters, we utilise the genetic algorithm PIKAIA (Charbonneau 1995), which is designed to find the set of parameters that minimizes the difference between the modelled and the reference or data sets. Here, we minimize the sum of the reduced chi-squared value $\left(\chi^{2}\right)$, in other words, we search for the minimum of $\chi_{\mathrm{TSI}}^{2}+\chi_{\text {faculae }}^{2}+\chi_{\mathrm{UV}}^{2}+\chi_{\mathrm{TMF}}^{2}+\chi_{\mathrm{OMF}}^{2}$ (Vieira and Solanki 2010). The results are described in Sect. 4.3.

\subsubsection{SATIRE-M}

As direct solar observations are not available before the Maunder minimum, to reconstruct the irradiance before that we use the concentrations of the cosmogenic isotopes ${ }^{14} \mathrm{C}$ and ${ }^{10} \mathrm{Be}$ in natural archives to describe the changes of solar activity with time.

\subsubsection{Cosmogenic data}

The cosmogenic radioisotopes ${ }^{14} \mathrm{C}$ and ${ }^{10} \mathrm{Be}$ are produced in the terrestrial atmosphere by galactic cosmic rays (GCRs). After entering the Earth's atmosphere, GCRs collide with nuclei (mainly Oxygen and Nitrogen) and produce these radionuclides. Since the GCR flux is modulated by the heliospheric magnetic field (e.g., Beer 2000a; Muscheler

\footnotetext{
${ }^{3}$ http: //www . pmodwrc.ch/pmod.php?topic=tsi/composite/SolarConstant; version d42_65_1608
} 


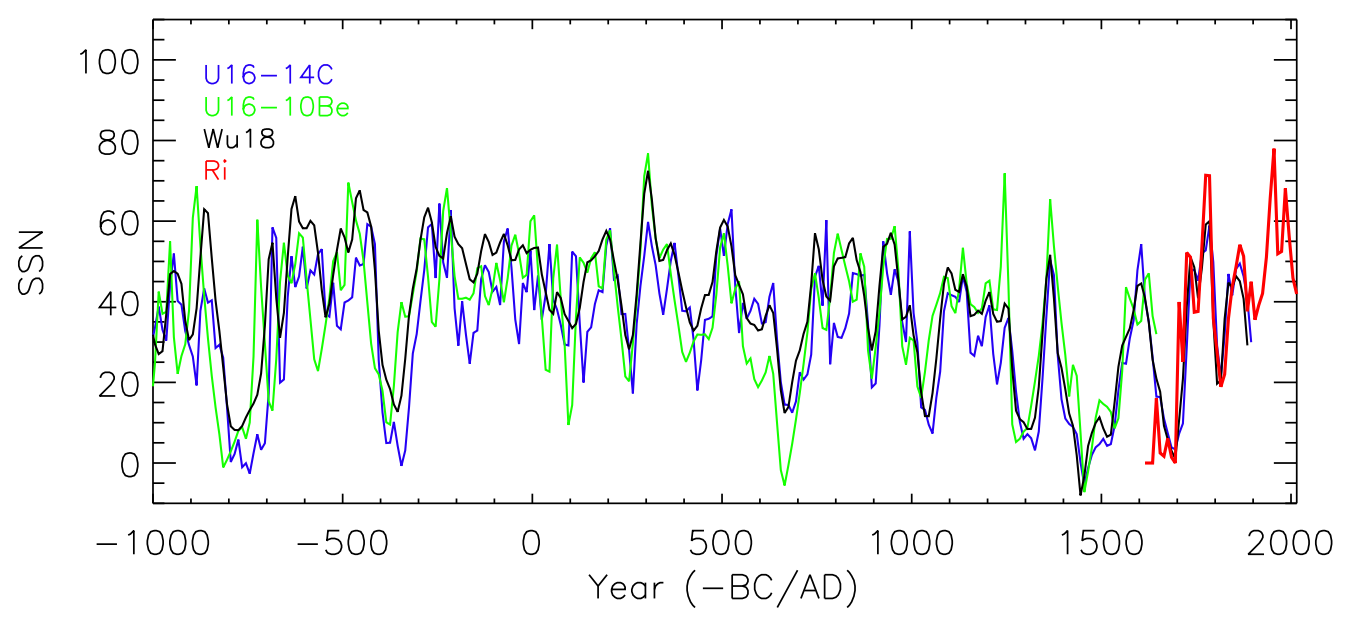

Figure 4.1: Sunspot series used as input in the SATIRE-M model over the period 1000BC to $2015 \mathrm{AD}$. U16-14C (blue): ${ }^{14} \mathrm{C}$ from Usoskin et al. (2016a); U16-10Be (green): ${ }^{10} \mathrm{Be}$ from Usoskin et al. (2016a); Wu18 (black): multi-isotope composite constructed by Wu et al. (2018b). The decadally-averaged directly observed sunspot number $R_{\mathrm{i}}$ (International sunspot number v2.0) used in this paper is compared in thick red line.

et al. 2004; Usoskin et al. 2006a; Steinhilber et al. 2008; Vieira et al. 2011), this allows a reconstruction of the solar magnetic activity at the time of the isotope production (Lal and Peters 1967).

To convert the concentrations of the cosmogenic isotopes into Sun-related quantities, several models have to be applied, such as the isotope production model (Masarik and Beer 1999; Kovaltsov et al. 2012; Poluianov et al. 2016), atmospheric transport and deposition model (e.g., Heikkilä et al. 2009; Roth and Joos 2013), and the geomagnetic field model (Yang et al. 2000; Korte and Constable 2005; Knudsen et al. 2008; Usoskin et al. 2016a).

To account for the geomagnetic field, two different models have been used: VDM (virtual dipole moment, Korte and Constable 2005) and VADM (virtual axial dipole moment, Usoskin et al. 2016a). VDM is approximated as a geocentric and tilted dipole and is reliable for only up to a few thousand years when the spread and quality of geomagnetic samples are good. VADM assumes the dipole to be aligned with the geographical axis and provides a rough geomagnetic estimation for the Holocene.

After production, ${ }^{14} \mathrm{C}$ takes part in the global carbon circulation in the atmosphere until it is absorbed by plants or stored in sediments (Roth and Joos 2013), while ${ }^{10} \mathrm{Be}$ attaches to aerosols and quickly precipitates within $1-2$ years after the production and is preserved in reservoirs or ice sheet (Vonmoos et al. 2006). Unlike ${ }^{14} \mathrm{C}$, which carries globally averaged information, ${ }^{10} \mathrm{Be}$ records are strongly regional-dependent (Sukhodolov et al. 2017). Therefore, the signals retrieved from various natural archives are affected by the geochemical process (Steinhilber et al. 2008, 2012; Beer et al. 2012), or the so-called systematic differences (e.g., snow accumulation rate, long-term changes in the carbon cycle, dating uncertainties).

In this study, three series of decadal SN reconstructed from cosmogenic isotopes are used, as described below. The first, ${ }^{14} \mathrm{C}$-based, SN series ("U16-14", Usoskin et al. 2016a) 
Table 4.2: Data sets used here, their time spans and the geomagnetic models.

\begin{tabular}{lccc|c}
\hline \hline Date set & Radionuclide & Data spans & SN version & Geomagnetic model \\
\hline $\mathrm{U} 16-14 \mathrm{C}$ & ${ }^{14} \mathrm{C}$ & $6755 \mathrm{BC}-1895 \mathrm{AD}$ & $\mathrm{U} 16$ & \\
$\mathrm{U} 16-10 \mathrm{Be}$ & ${ }^{10} \mathrm{Be}$ & $6755 \mathrm{BC}-1645 \mathrm{AD}$ & $\mathrm{U} 16$ & \multirow{2}{*}{ VADM; U16 } \\
$\mathrm{Wu} 18$ & ${ }^{14} \mathrm{C} \mathrm{\&}{ }^{10} \mathrm{Be}$ & $6755 \mathrm{BC}-1895 \mathrm{AD}$ & $\mathrm{Wu} 18$ & \\
\hline
\end{tabular}

References. U16: Usoskin et al. (2016a); Wu18: Wu et al. (2018b).

is an extended and updated version of a series taken from Usoskin et al. (2014). It is calculated from an updated ${ }^{14} \mathrm{C}$ production rate record INTCAL09 (Reimer et al. 2009) and a new geomagnetic model (GMAG.9k) reconstructed by Usoskin et al. (2016a). Based on the geomagnetic model GMAG.9k, Usoskin et al. (2016a) also reconstructed a SN series ("U16-10Be") from the longest ${ }^{10} \mathrm{Be}$ data set from the Greenland Ice Core Project (GRIP; Yiou et al. 1997; Muscheler et al. 2004; Vonmoos et al. 2006).

Figure 4.1 shows the SN series U16-14C (blue) and U16-10Be (green) over the period 1000 BC to 2015 AD. Due to the different geochemical production processes and nonlinear local climatic influences, some temporal discrepancies between multiple isotope data sets can be found (e.g., 900 BC - 700 BC). Several studies (Delaygue and Bard 2011; Steinhilber et al. 2012) have combined the individual series using a normalization and the Principal Component Analysis (PCA), which extract the common signals (assumed to be solar) and remove the systematic effects. However, this method keeps only the relative variability, and essentially averages the data points at a given time, without taking the geomagnetic fields and the potential time lags into account and neglecting uncertainties. According to Adolphi and Muscheler (2016), the time lags between individual series may reach up to several decades. To account for these issues, Wu et al. (2018b) combined six ${ }^{10} \mathrm{Be}$ series with the global ${ }^{14} \mathrm{C}$ record into a consistent multi-isotope series, "Wu18", the third series used in this study. This composite takes care of the temporal discrepancies between the seven individual series and minimises the difference between the modelled solar activity and the measured cosmogenic isotope data (see Wu et al. 2018b, for more details), as shown by the thick black curve in Fig. 4.1. For comparison, the decadallyaveraged directly observed sunspot number series after 1639 is represented by the red solid line. These data sets, their periods, and the geomagnetic field used are summarized in Table 4.2.

Until now, the reconstructions of TSI were only based on individual records (Shapiro et al. 2011; Delaygue and Bard 2011; Vieira et al. 2011), of which only one reconstruction (Vieira et al. 2011) was made with a non-linear model. We here make use of the recent composite, Wu18, to reconstruct the TSI and the first SSI series over the Holocene.

\subsubsection{Reconstruction of solar irradiance}

The quantity related to solar activity that can be derived most directly from cosmogenic isotope concentrations is the solar modulation potential. It describes the energy loss of GCRs in the heliosphere due to the solar magnetic field (Usoskin et al. 2005). The solar modulation potential can then be converted into the solar open magnetic flux (OMF) using the heliospheric cosmic ray transport model (Usoskin et al. 2007). 
As described by Eqs. (4.2) - (4.7), the open magnetic flux is related to ARs and ERs. Vieira and Solanki (2010) and Vieira et al. (2011) have made use of this relationship, as well as the other two physical assumptions, see the descriptions below, to take the lower temporal resolution of the cosmogenic isotope data into account. This modified model is termed SATIRE-M model. The first physical assumption is that the fluxes in ARs, ERs and rapidly evolving open flux are in a steady state on decadal time scale, i.e., the emerging flux is approximately equal to the decaying flux (Vieira and Solanki 2010):

$$
\left\langle\frac{\varphi_{\mathrm{act}}}{\tau_{\mathrm{act}}}\right\rangle=\left\langle\varepsilon_{\mathrm{act}}\right\rangle, \quad\left\langle\frac{\varphi_{\mathrm{eph}}}{\tau_{\mathrm{eph}}}\right\rangle=\left\langle\varepsilon_{\mathrm{eph}}\right\rangle, \quad\left\langle\frac{\varphi_{\mathrm{act}}}{\tau_{\mathrm{act}}^{r}}\right\rangle=\left\langle\frac{\varphi_{\mathrm{open}}^{r}}{\tau_{\mathrm{open}}^{r}}\right\rangle,
$$

where $\langle\ldots\rangle$ represents decadal average. The temporal parameters $\tau_{\text {act }}$ and $\tau_{\mathrm{eph}}$ are

$$
\begin{gathered}
\frac{1}{\tau_{\mathrm{act}}}=\frac{1}{\tau_{\mathrm{act}}^{0}}+\frac{1}{\tau_{\mathrm{act}}^{\mathrm{s}}}+\frac{1}{\tau_{\mathrm{act}}^{\mathrm{r}}}, \\
\frac{1}{\tau_{\mathrm{eph}}}=\frac{1}{\tau_{\mathrm{eph}}^{0}}+\frac{1}{\tau_{\mathrm{eph}}^{\mathrm{s}}} .
\end{gathered}
$$

The second physcial assumption is that the decadally-averaged sunspot number is linearly related to the maximum sunspot number of the corresponding cycle (i.e., $\langle R\rangle \propto R_{i}^{\max }$, Usoskin et al. 2007; Vieira and Solanki 2010). Based on these assumptions, Vieira and Solanki (2010) obtained the following relationship between the decadal sunspot number and the solar open flux:

$$
\left\langle R_{\mathrm{i}}\right\rangle_{j}=a_{\mathrm{R}}\left\langle\varphi_{\mathrm{open}}\right\rangle_{j}+b_{\mathrm{R}}\left\langle\varphi_{\mathrm{open}}\right\rangle_{j+1},
$$

where $a_{\mathrm{R}}=1 / c \tau_{1}$ and $b_{\mathrm{R}}=1 / c \Delta t$, respectively. The sampling interval $\Delta t$ is 10 years, and the constant $c$ is

$$
c=\left[\left(\frac{1}{\tau_{\mathrm{act}}^{\mathrm{s}}}+\frac{\tau_{\mathrm{open}}^{\mathrm{r}}}{\tau_{\mathrm{open}}^{\mathrm{s}} \tau_{\mathrm{act}}^{\mathrm{r}}}\right) \tau_{\mathrm{act}}+\frac{2.2 X \tau_{\mathrm{eph}}}{\tau_{\mathrm{eph}}^{\mathrm{s}}}\right] \frac{\varepsilon_{\mathrm{act}}^{\mathrm{max}, 21}}{R_{\mathrm{i}}^{\mathrm{max}, 21}},
$$

and the time parameter $\tau_{1}$ is

$$
\frac{1}{\tau_{1}}=\frac{1}{\tau_{\mathrm{open}}^{\mathrm{s}}}-\frac{1}{\Delta t}
$$

The time parameters $\tau_{1}, \tau_{\text {act }}, \tau_{\text {eph }}$ and the ER amplitude factor $X$ are taken from the SATIRE-T model and are fixed here.

The equation (4.14) describes the relation between decadally averaged SN and the OMFs from two cycles. Physically, this relationship means that the open flux during a given cycle is affected by solar activity from this and the previous solar cycle. Therefore, the ratio of the contributions from two cycles, $a_{\mathrm{R}} / b_{\mathrm{R}}=\Delta t / \tau_{1}=\left(\Delta t / \tau_{\text {open }}^{\mathrm{s}}\right)-1$, depends on the sampling interval and on the decay time of slowly evolving open flux. In this study, our sampling interval and the $\tau_{\text {open }}^{\mathrm{s}}$ are 10 years and 3.75 years, respectively. Thus, the $a_{\mathrm{R}} / b_{\mathrm{R}}$ ratio is 1.67 , which is lower than the estimate by Vieira et al. (2011), who obtained 2.4. This implies that the contribution of the previous cycle to the open flux at a given time is higher in the updated model.

Next, to calculate the solar irradiance, the filling factors of all magnetic components are needed (see Eq. 4.1). We first compute the filling factors for the sunspot umbrae and 
penumbrae. The relation between the decadal sunspot area (decadally-averaged filling factors for umbrae and penumbrae) and the decadal sunspot number can be simplified into a linear relation (Vieira et al. 2011):

$$
\left\langle\alpha_{\mathrm{s}}\right\rangle_{j}=A_{1}\left\langle R_{\mathrm{i}}\right\rangle_{j}+A_{2}
$$

where $A_{1}$ is a proportionality coefficient and $A_{2}$ is the offset. Both coefficients are timeindependent, as well as the various $a, b$ and $c$ parameters appearing in equations below. Filling factors for umbra and penumbra are obtained by combining Eqs. (4.14) and (4.17) and assuming a ratio of umbral to penumbral area of 1:4 (Solanki 2003):

$$
\begin{aligned}
& \left\langle\alpha_{\mathrm{u}}\right\rangle_{j}=a_{\mathrm{u}}\left\langle\varphi_{\text {open }}\right\rangle_{j}+b_{\mathrm{u}}\left\langle\varphi_{\text {open }}\right\rangle_{j+1}+c_{\mathrm{u}}, \\
& \left\langle\alpha_{\mathrm{p}}\right\rangle_{j}=a_{\mathrm{p}}\left\langle\varphi_{\text {open }}\right\rangle_{j}+b_{\mathrm{p}}\left\langle\varphi_{\text {open }}\right\rangle_{j+1}+c_{\mathrm{p}} .
\end{aligned}
$$

The values of $a_{\mathrm{u}, \mathrm{p}}, b_{\mathrm{u}, \mathrm{p}}$ and $c_{\mathrm{u}, \mathrm{p}}$ used in this study are listed in Table 4.3.

The filling factors for faculae and network are found similarly to SATIRE-T while taking the decadal average into account. Thus, we assume (1) the mean field strength of umbra $\left(\left[B_{\mathrm{u}}\right]\right)$ and penumbra $\left(\left[B_{\mathrm{p}}\right]\right)$ are $1800 \mathrm{G}$ and $550 \mathrm{G}$, respectively; (2) an active region is composed of sunspot and faculae; and (3) the network includes ERs and OMF, i.e., $\varphi_{\mathrm{n}}=\varphi_{\mathrm{eph}}+\varphi_{\mathrm{open}}$ (Krivova et al. 2010). The filling factors of faculae and network can then be written as

$$
\begin{gathered}
\left\langle\alpha_{\mathrm{f}}\right\rangle_{j}=\frac{\left\langle\varphi_{\mathrm{f}}\right\rangle_{j}}{S_{\odot} B_{\text {sat }, \mathrm{f}}}=a_{\mathrm{f}}\left\langle\varphi_{\mathrm{open}}\right\rangle_{j}+b_{\mathrm{f}}\left\langle\varphi_{\mathrm{open}}\right\rangle_{j+1}+c_{\mathrm{f}}, \\
\left\langle\alpha_{\mathrm{n}}\right\rangle_{j}=\frac{\left\langle\varphi_{\mathrm{n}}\right\rangle_{j}}{S_{\odot} B_{\text {sat }, \mathrm{n}}}=a_{\mathrm{n}}\left\langle\varphi_{\text {open }}\right\rangle_{j}+b_{\mathrm{n}}\left\langle\varphi_{\text {open }}\right\rangle_{j+1},
\end{gathered}
$$

where $S_{\odot}$ is the solar surface area. The magnetic saturation limits of faculae $\left(B_{\text {sat, }}\right)$ and network $\left(B_{\mathrm{sat}, \mathrm{n}}\right)$ are adapted from SATIRE-T as well. The values of $a_{\mathrm{f}, \mathrm{n}}, b_{\mathrm{f}, \mathrm{n}}$ and $c_{\mathrm{f}}$ used in this study are listed in Table 4.3.

Finally, the solar spectral irradiance can be obtained by applying these filling factors of the magnetic components in the Eq. (4.1):

$$
\langle F(\lambda, t)\rangle_{j}=a_{\mathrm{F}}(\lambda)\left\langle\varphi_{\mathrm{open}}\right\rangle_{j}+b_{\mathrm{F}}(\lambda)\left\langle\varphi_{\mathrm{open}}\right\rangle_{j+1}+F_{\mathrm{q}}(\lambda)
$$

where

$$
\left(a_{\mathrm{F}}, b_{\mathrm{F}}\right)(\lambda)=\sum_{i=\mathrm{u}, \mathrm{p}, \mathrm{f}, \mathrm{n}}\left(a_{i}, b_{i}\right)\left\langle F_{i}(\lambda)-F_{\mathrm{q}}(\lambda)\right\rangle .
$$

It is important to note that with the Eq. (4.22), the solar spectral and total irradiance can be computed straightforwardly from the values of the open flux from two consecutive cycles. Moreover, during the simplification of the SATIRE-M model in order to deal with the decadal cadence, the model heritages all the parameters from the SATIRE-T model (Sect. 4.1) and no free parameters are introduced. 
Table 4.3: Parameters used in Eqs. (4.18) - (4.21).

\begin{tabular}{ccccc}
\hline \hline Quantity & $\begin{array}{c}\mathrm{a} \\
{\left[10^{-5} \mathrm{~Wb}^{-1}\right]}\end{array}$ & $\begin{array}{c}\mathrm{b} \\
{\left[10^{-5} \mathrm{~Wb}^{-1}\right]}\end{array}$ & $\mathrm{c}$ & $\mathrm{a} / \mathrm{b}$ \\
\hline$\alpha_{\mathrm{u}}$ & 3.2 & 1.9 & -0.4 & 1.67 \\
$\alpha_{\mathrm{p}}$ & 12.8 & 7.7 & -1.6 & 1.67 \\
$\alpha_{\mathrm{f}}$ & 126.3 & 75.7 & -9.1 & 1.67 \\
$\alpha_{\mathrm{n}}$ & 71.8 & 30.8 & - & 2.33 \\
\hline
\end{tabular}

\subsection{Results}

\subsubsection{Comparison with observations}

As described in Sect. 4.2.2.3, we fix the free parameters of the SATIRE-T model by comparing the output with five independent data records. Here, we first compare the reconstructed quantities to the corresponding observations or alternative models over their overlapping periods of time.

The modelled total magnetic flux (TMF) is compared with the observations in Fig. 4.2. Panel a shows the TMF over cycles $21-23$. Data from different observatories are represented by different symbols: WSO (squares), KP NSO (diamonds) and MWO (triangles). Each individual symbol represents the total photospheric magnetic flux for a given Carrington rotation. The thick solid line is a 3-month moving average of the reconstruction. The detectable ER flux is reduced by a factor of 0.4 to take the underdetected flux in observations into account. The figure shows the data since 1967, but we only calculate the reduced $\chi^{2}$ value from 1976 onwards, i.e., to the right of the vertical dashed line, when all three observational data sets are available. In contrast to the previous work by Krivova et al. (2010, Kea10 hereafter), who fitted the modelled TMF to individual observation data points, we fit to the average of the three, as indicated by the red line. The reduced $\chi^{2}$ value is 0.036 . Panel b compares the modelled OMF (solid line, yearly averages) with the empirical reconstruction by Lockwood et al. (2014a, dashed line) based on the $a a$-index between $1850-2010$. The reduced $\chi^{2}$ value over this period is 0.225 . Note that although the reference open magnetic flux record used in this paper covers 60 years longer than the previous version (Lockwood et al. 2009) used by Kea10, our modelled has a lower $\chi^{2}$ value than in Kea10 $\left(\chi^{2}=0.243\right)$.

Figure 4.3a shows the TSI reconstruction (red dots) from the sunspot number $\left(R_{\mathrm{i}}\right)$ overplotted on the PMOD composite (green lines) over the period $1978-2015$. In this study, we take the SORCE/TIM measurement during the activity minimum in 2008 $\left(1360.52 \mathrm{~W} / \mathrm{m}^{2}\right)$ as reference. Both, the reconstructed TSI and PMOD series are normalized to the 2008 minimum level, indicated by the dashed horizontal line. The difference between the two series is shown in panel $b$ (daily values as dots and the 361-day moving average as thick solid line). The reduced $\chi^{2}$ value between the daily modelled TSI and the PMOD composite is 0.20 , which is lower than 0.233, obtained by Kea10.

Next, we compare the modelled UV flux (integral over $220-240 \mathrm{~nm}$; red) with the reference data (black) in Fig. 4.4a. The obtained $\chi^{2}$ value of 0.043 is significantly lower than the value obtained by Kea10. The reference UV time series here is the reconstruction with SATIRE-S model which has been shown to be in close agreement $\left(R_{\mathrm{c}}^{2}=0.975\right)$ with 

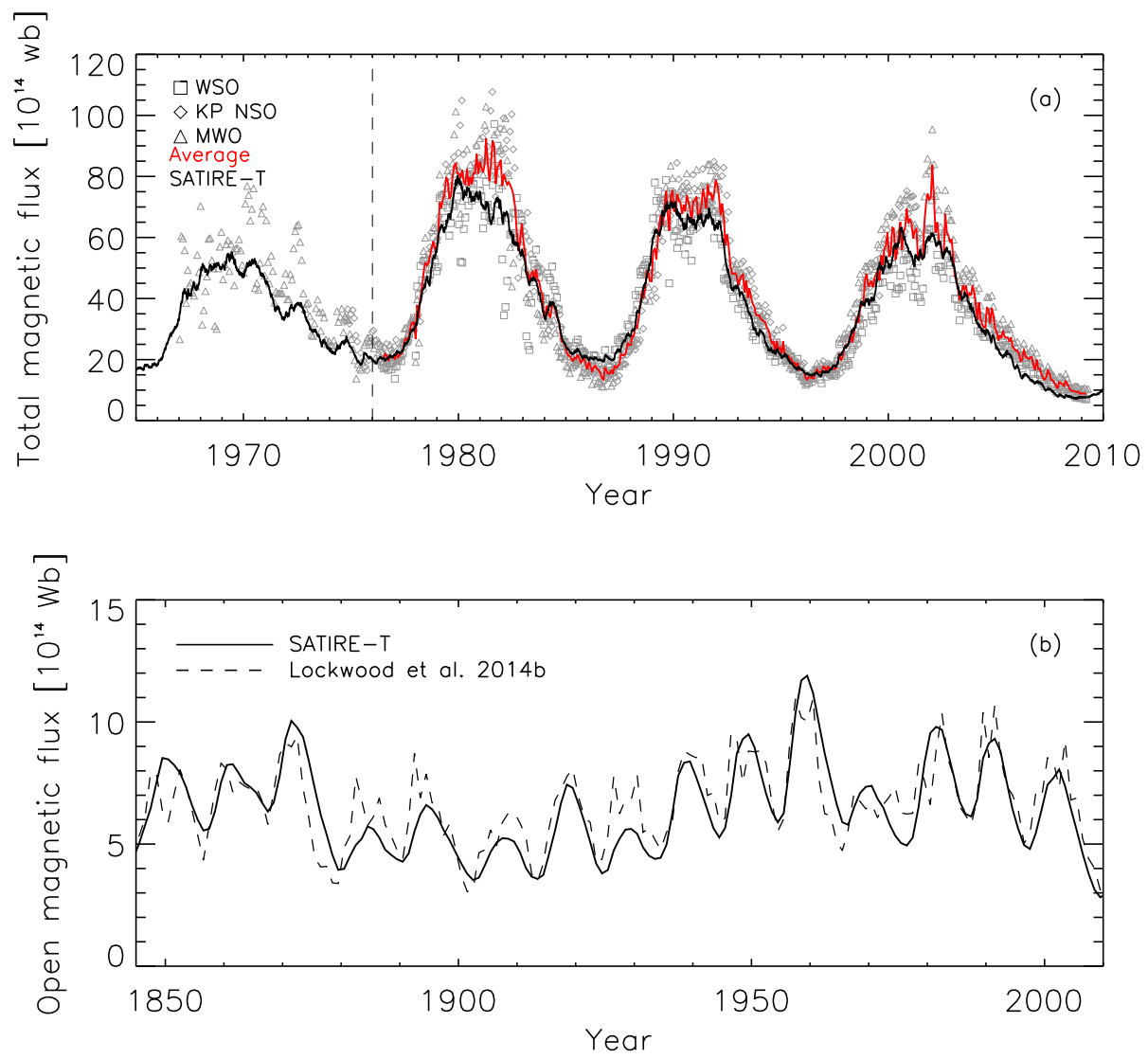

Figure 4.2: a) 3-month moving averaged reconstructed total magnetic flux (thick black line) compared to the averages of the three observational data sets (thick red line). The three individual observational data sets are WSO (squares), KP NSO (diamonds) and MWO (triangles). The reconstructed total magnetic flux shown is $\varphi_{\text {tot }}=\varphi_{\text {act }}+0.4 \varphi_{\mathrm{eph}}+$ $\varphi_{\text {open }}$, to take the unresolved magnetic flux in small ephemeral regions into account (see Sect. 4.2.2.3). b) Yearly averaged reconstructed OMF (solid line) since 1845 and the reconstruction from the $a a$-index by Lockwood et al. (2014a, dashed line).

the UARS/SUSIM record. We take the SATIRE-S reconstruction here rather than the SUSIM data, since it covers a much longer period (11 years vs. 40 years, Krivova et al. 2006; Yeo et al. 2014b).

Since the irradiance variations in the UV range are mainly due to faculae (Unruh et al. 2008), we additionally constrain the SATIRE-T model by requiring that it also reproduces the facular contribution to the TSI variation correctly. The calculated facular contribution to the TSI variation over the period $1974-2015$ (red) is compared to that obtained from the more accurate SATIRE-S model based on full-disc solar magnetograms (black, Yeo et al. 2014b) in Fig. 4.4b. Since the direct information on the emergence rate of bright features (faculae and network) back to 1610 is missing, their emergence rate is assumed to be related to the evolution of sunspots. This is a valid assumption on time scale of months or longer, but the modelled facular variability cannot be expected to be accurate on time scales shorter than a month. Therefore, the facular contribution is shown as an 81 -day moving average. The reduced $\chi^{2}$ value between the two series is 0.018 , which is 
Table 4.4: The reduced $\chi^{2}$ values and the correlation coefficients $\left(R_{\mathrm{c}}\right)$ between the SATIRE-T reconstruction and the independent data sets used to constrain the model (except the Lyman- $\alpha$, which was not used for fixing the parameters).

\begin{tabular}{cccccccc}
\hline \hline $\begin{array}{c}\text { Quantity } \\
\text { Version }\end{array}$ & Cadence & Period & $\begin{array}{c}\chi^{2} \\
\text { Kea10 }\end{array}$ & $\begin{array}{c}\chi^{2} \\
\text { This work }\end{array}$ \\
\hline TSI & $1 \mathrm{~d}$ & $1978-2015$ & 0.233 & 0.81 & 0.200 & 0.80 \\
TMF & $1 \mathrm{CR}^{a}$ & cycle $21-23$ & 0.069 & 0.93 & 0.036 & 0.98 \\
OMF & $1 \mathrm{y}$ & $1845-2010$ & 0.248 & 0.86 & 0.243 & 0.82 \\
& $3 \mathrm{~m}$ & $1974-2015$ & 0.064 & 0.94 & 0.018 & 0.97 \\
$\begin{array}{c}\text { Facular contribution to } \\
\text { the TSI variation }\end{array}$ & & & & & & \\
UV (220-240 nm) & $3 \mathrm{~m}$ & $1947-2015$ & 0.072 & 0.94 & 0.043 & 0.96 \\
\hline Lyman- $\alpha$ & $3 \mathrm{~m}$ & $1947-2015$ & - & - & 0.13 & 0.96
\end{tabular}

Notes. ${ }^{(a)}$ Carrington rotation.

almost 4 times lower than in Kea10. The great improvement in the UV-band is due to the more accurate description of the activity belts (see Sect. 4.2.2.2). It is also worth noticing that both the secular change and the solar cycle amplitude of the UV fluxes and the facular contribution to the TSI changes agree very well with the SATIRE-S model.

After constraining the SATIRE-T parameters by comparing to the various mentioned independent data sets above, we also compare the modelled Lyman- $\alpha$ irradiance with the LASP composite of measurements (since 1978) and a proxy model (prior to 1978, Woods et al. 2000, 2004) ${ }^{4}$. The Lyman- $\alpha$ line impacts the chemistry and heating rates in the terrestrial middle atmosphere and is, therefore, of interest for climate research. A comparison over the period 1947 - 2015 in shown in Fig. 4.5, the modelled Lyman- $\alpha$ is in red and the composite is in black. The 1- $\sigma$ uncertainty of the composite is indicated in gray shade. The modelled Lyman- $\alpha$ is normalized to the 2008 minimum (horizontal dashed line). The reduced $\chi^{2}$ is 0.013 . We emphasize that this data set was not used to constrain the model, so that the good agreement between the model and the data provides further support to our model.

In summary, the resulting reconstructions show excellent agreement with the corresponding reference data. The derived free parameters are listed in Table 4.1. The reduced $\chi^{2}$ values and the correlation coefficients $\left(R_{\mathrm{c}}\right)$ of the physical quantities are summarized in Table 4.4, compared with the results of Kea10. The parameters are now fixed and further employed to reconstruct the solar spectral irradiance over the last nine millennia (Sect. 4.2.3).

\subsubsection{SATIRE-T reconstruction back to the Maunder minimum}

Figure 4.6a shows the 361-day smoothed reconstructions of the TMF (thick-solid line), AR flux (red), ER flux (blue) and OMF (dashed). Note that the TMF is without the reduction by $c_{\mathrm{e}}=0.4$, as here we are interested in the total flux responsible for irradiance changes, not the one observed. In our reconstruction, the ER cycles are shorter than the previous versions. It is important to note that while the reconstructed fluxes during the

\footnotetext{
${ }^{4}$ http://lasp.colorado.edu/lisird/lya/
} 


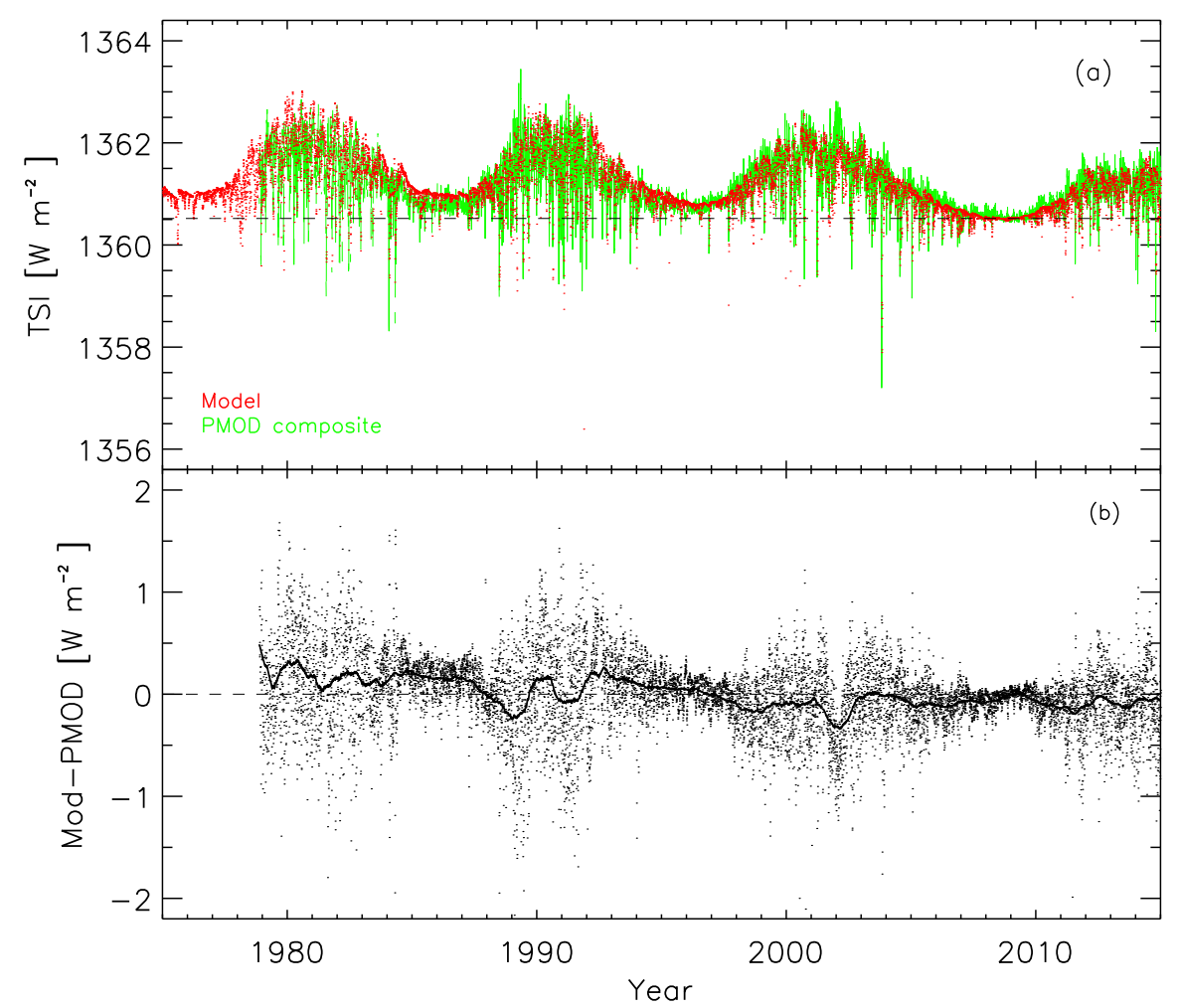

Figure 4.3: TSI since 1978: a) Daily reconstructed (red) and measured PMOD composite (green). b) Difference between the reconstruction and the PMOD composite. Daily values are indicated by dots and the 361-day moving average by the thick solid line.

Maunder minimum drop down to zero, this does not imply that the solar activity was completely absent at that time. It is only a consequence of the lack of information on solar activity at that time. Since the sunspot number dropped to zero, the emergence rates of magnetic regions in our model are also zero over most of that period. In principle, the magnetic flux might have still emerged in some weaker regions, and contributed to the OMF, which is supported by the cosmogenic data (Stuiver and Braziunas 1993; Kocharov et al. 1995; Beer et al. 1998). This means that the irradiance drop during the Maunder minimum was probably somewhat lower than our model returns, and the estimated secular change is thus an upper limit.

The reconstructed TSI (SSI integrated over $115-160000 \mathrm{~nm}$ ) is shown in Fig. 4.6b (361-day moving average in black, 11-year running mean in blue). The dashed horizontal line indicates the Maunder minimum level of TSI. To compare with other studies, we calculate the increase in the TSI from the end of the Maunder minimum to the mean over the period $1975-2005$. Our reconstruction returns an increase of $0.92_{-0.02}^{+0.08} \mathrm{~W} / \mathrm{m}^{2}$, which is somewhat lower, although still within the uncertainty ranges, than in the previous reconstructions with the SATIRE-T model $\left(1.25_{-0.3}^{+0.05} \mathrm{~W} / \mathrm{m}^{2}\right.$, Krivova et al. 2007, 2010), SATIRE-T2, based on synthetic magnetograms produced with a surface flux transport model from the group sunspot number $\left(1.2_{-0.3}^{+0.2} \mathrm{~W} / \mathrm{m}^{2}\right.$, Dasi-Espuig et al. 2016), and the empirical reconstruction with the NRLTSI model $\left(1.1 \mathrm{~W} / \mathrm{m}^{2}\right.$, Coddington et al. 2016).

Since the sunspot number is the only input to the SATIRE-T model, the choice of the 

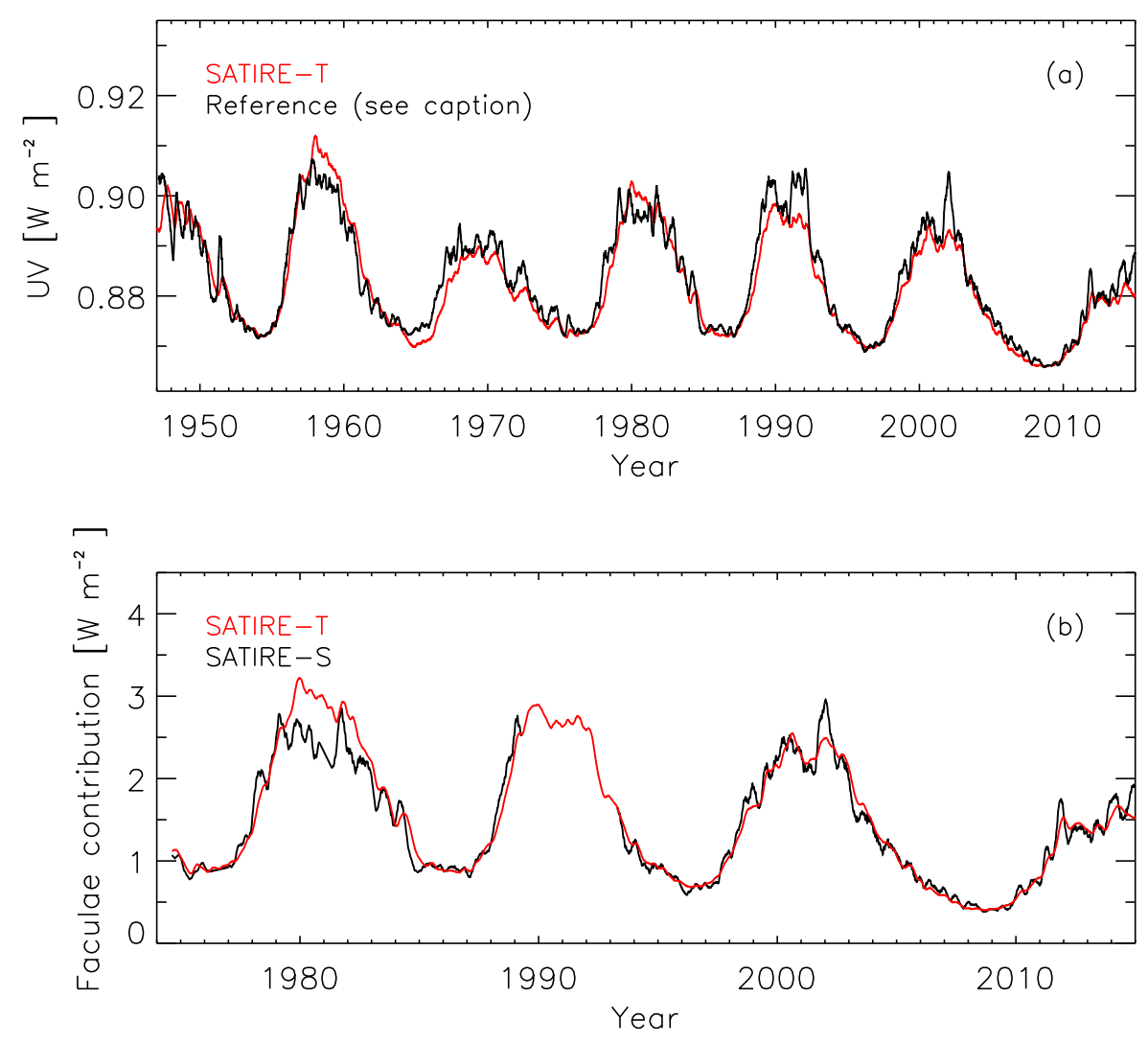

Figure 4.4: a) The reconstructed UV irradiance $(220-240 \mathrm{~nm}$, red) and the reference UV data set (Yeo et al. 2014b, black); b) the facular contribution to the TSI changes modelled with SATIRE-T (red) and obtained from full-disc solar magnetograms (SATIRE-S; black). In the SATIRE-S model the period between 1990 - 1993 is not shown due to gaps in the input data (Yeo et al. 2014b). All curves are in 81-day moving averages.

sunspot number series affects the level in the modelled solar irradiance during the 18th to early 19th century. However, the secular change is not affected (c.f., Kopp 2016), since neither the modern nor the Maunder minimum levels are affected by this choice. One of the shortcomings of most existing sunspot number reconstructions is the employment of a linear scaling between various individual records, which is not justified (Usoskin 2017). Among the proposed revised sunspot number series (Sect. 4.2.2.1), Usoskin et al. (2016c) and Chatzistergos et al. (2017) were the only ones to apply a non-linear non-parametric calibration method. Chatzistergos et al. (2017) did the calibration using probability distribution functions (PDF) of several backbone observers with errors estimated with Monte Carlo simulations. Therefore we also consider their series here as an alternative input record to the SATIRE-T model. The difference between the TSI reconstructions from the sunspot number records by WDC-SILSO and Chatzistergos et al. (2017) is used here as a rough estimate of the uncertainty range over $1739-2010$ due to the choice of the sunspot number (gray shading in Fig. 4.6b).

To test the stability of our reconstruction and the sensitivity of the model to various free parameters, we have considered different combinations of parameters (e.g., fixed/free 


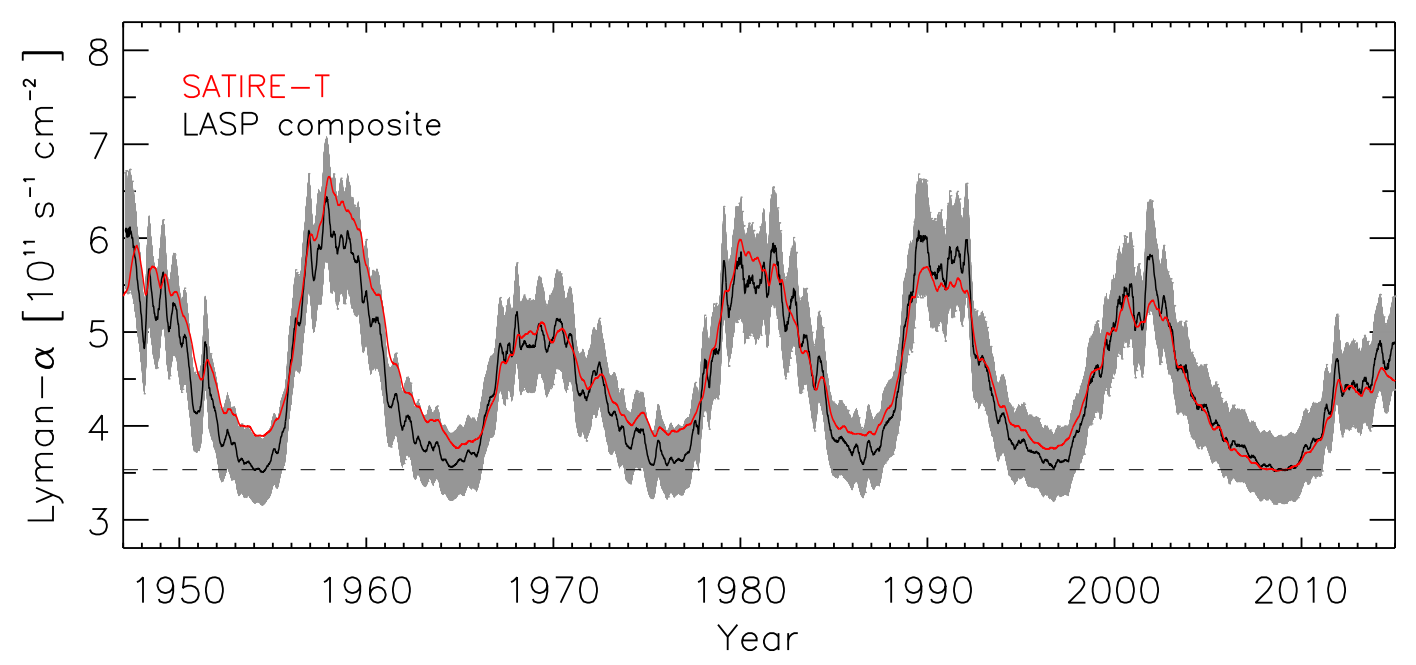

Figure 4.5: 81-day moving average of the reconstructed Lyman- $\alpha$ (red) and the Lyman- $\alpha$ composite by (Woods et al. 2004, see text) with its 1- $\sigma$ uncertainty (gray). The model is normalized to the composite during the 2008 solar minimum (dashed horizontal line).

$\tau_{\text {act }}^{0}$, fixed/free $B_{\text {sat, }}$ and fixed/free $c_{\mathrm{e}}$ ). These tests showed that the resulting reconstruction is stable to the choice of parameters. The overall secular change always lies in the range $0.90-1.0 \mathrm{~W} / \mathrm{m}^{2}$.

\subsubsection{SATIRE-M reconstruction on millennial time scales}

Keeping all the parameters fixed, we now employ the SATIRE-M model to reconstruct solar total and spectral irradiance back to $6755 \mathrm{BC}$ from the cosmogenic isotope data. Figure 4.7a shows the TSI reconstructions over the last 9000 years based on the three isotope series, as introduced in Sect. 4.2.3 (U16-14C in blue, U16-10Be in green, Wu18 in black). Figure $4.7 \mathrm{~b}$ is an enlargement over the period $1000 \mathrm{BC}-2000 \mathrm{AD}$. The decadallyaveraged TSI reconstruction based on the $R_{\mathrm{i}}$ series is also shown (red) for comparison.

It is worth noticing that the reconstructions based solely on either ${ }^{14} \mathrm{C}$ (U16-14C) or ${ }^{10} \mathrm{Be}(\mathrm{U} 16-10 \mathrm{Be})$ show temporal discrepancies due to differences in the relevant geochemical production processes and local climatic influences (Sect. 4.2.3.1 and $\mathrm{Wu}$ et al. 2018 b). The reconstruction based on the composite (Wu18) of one global ${ }^{14} \mathrm{C}$ and six regional ${ }^{10} \mathrm{Be}$ series has remedied this (See Fig. 12 in Wu et al. 2018b). The compositebased reconstruction shows the common solar signal extracted from both individual isotope series, and is therefore considered to be much more robust. On the millennial time scale, the variability of TSI ( $\triangle \mathrm{TSI} / \overline{\mathrm{TSI}})$ obtained from all three isotope records is very similar $(\approx 0.11 \%)$. Since in the second half of the 20th century, the activity of the Sun was comparatively high (see also Solanki et al. 2004), this result means that during extended activity minima (grand minima) the irradiance dropped by up to $1.5 \mathrm{~W} / \mathrm{m}^{2}$ compared to the recent level. Interestingly, the TSI over the 19th centuries level reconstructed from the cosmogenic isotope data agrees better with the reconstruction from the sunspot record by Chatzistergos et al. (2017) than that from the WDC-SILSO.

Figure 4.8 shows the reconstructed SSI in selected wavelength bands over the period 1000 BC - 2000 AD. The four selected spectral intervals are (a) Lyman- $\alpha$, here integral 


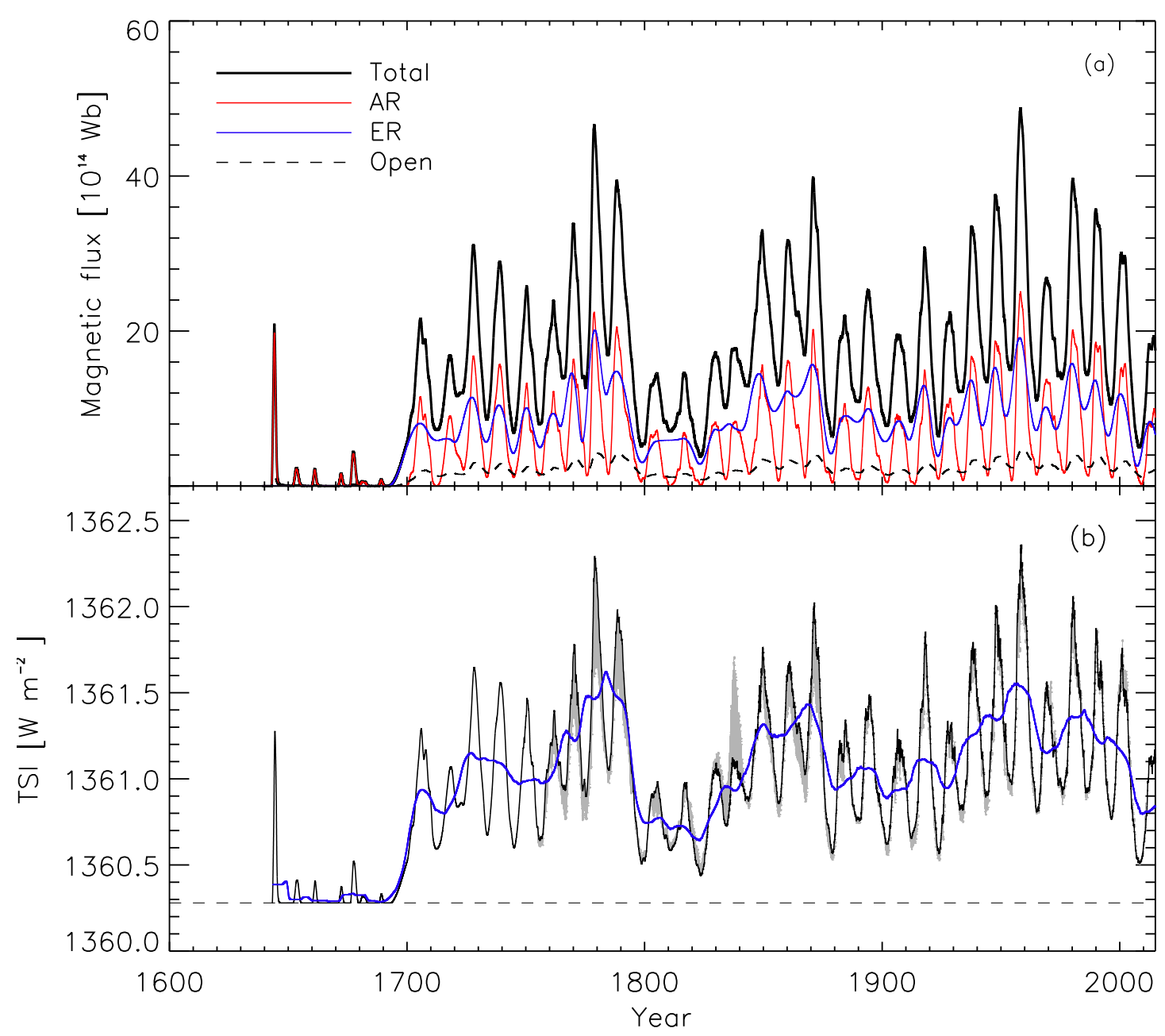

Figure 4.6: a) Reconstructed magnetic flux (361-day moving averages) at the solar surface since 1639: total magnetic flux (thick black), AR flux (red), ER flux (blue) and open flux (dashed). b) Reconstructed TSI back to 1639 (361-day moving averages in black and 11year smooth in blue). The horizontal dashed line shows the Maunder minimum level. The gray shaded area represents the uncertainty range due to the choice of the sunspot record (see main text for details). 

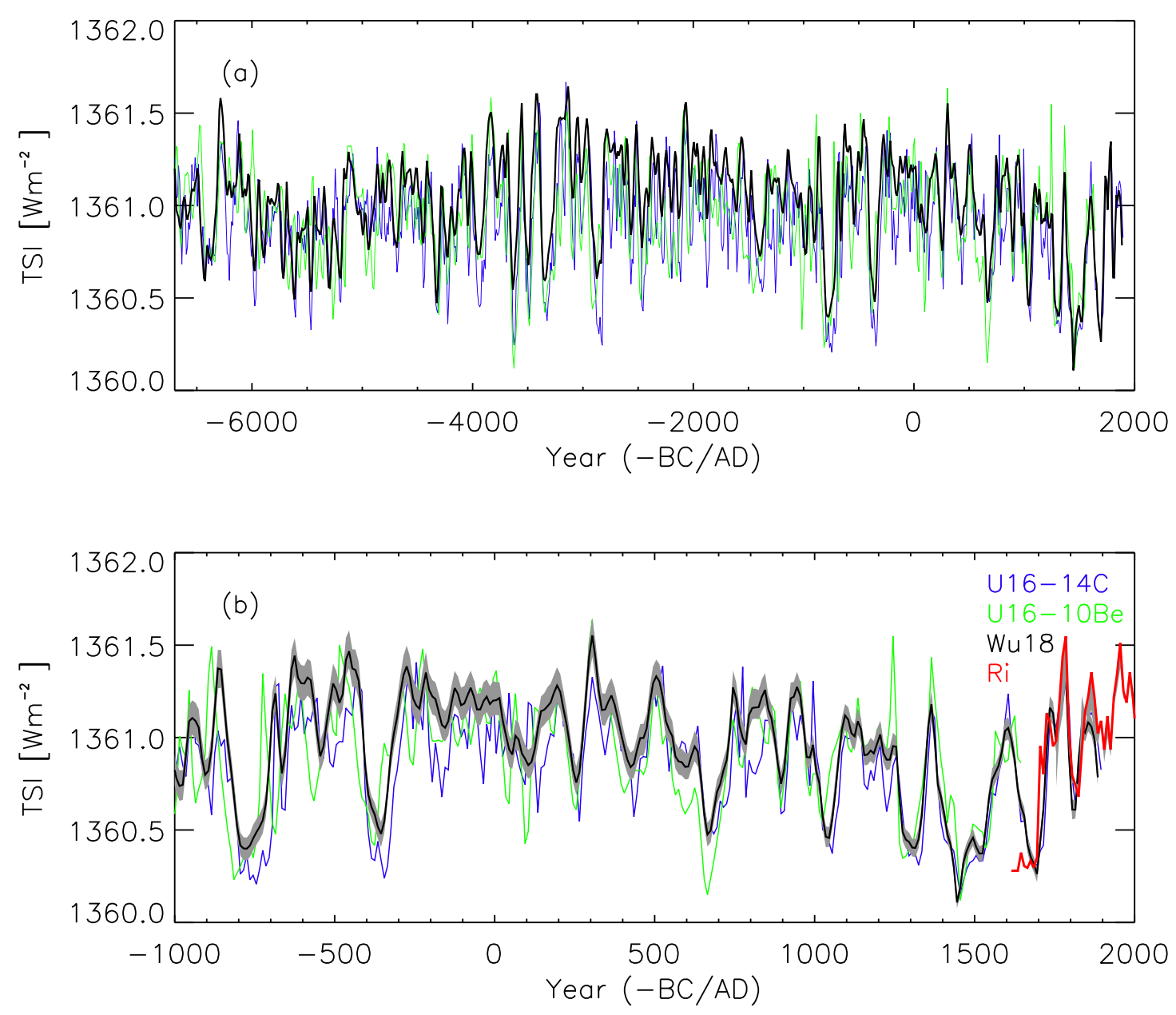

Figure 4.7: Three TSI reconstructions based on different isotope records: U16-14C (blue), U16-10Be (green) and the composite Wu18 (black) plotted over the periods: a) $6755 \mathrm{BC}$ $-2000 \mathrm{AD}$ and b) $1000 \mathrm{BC}-2000 \mathrm{AD}$. The uncertainty range of the reconstruction based on the Wu18 composite is shown in shaded area. Decadal averages of the reconstruction based on the directly observed SN are shown in red after 1639.

over 121 - $122 \mathrm{~nm}$, (b) UV between 115 - $400 \mathrm{~nm}$, (c) visible between 400 - $700 \mathrm{~nm}$ and (d) IR, longwards of $700 \mathrm{~nm}$. 

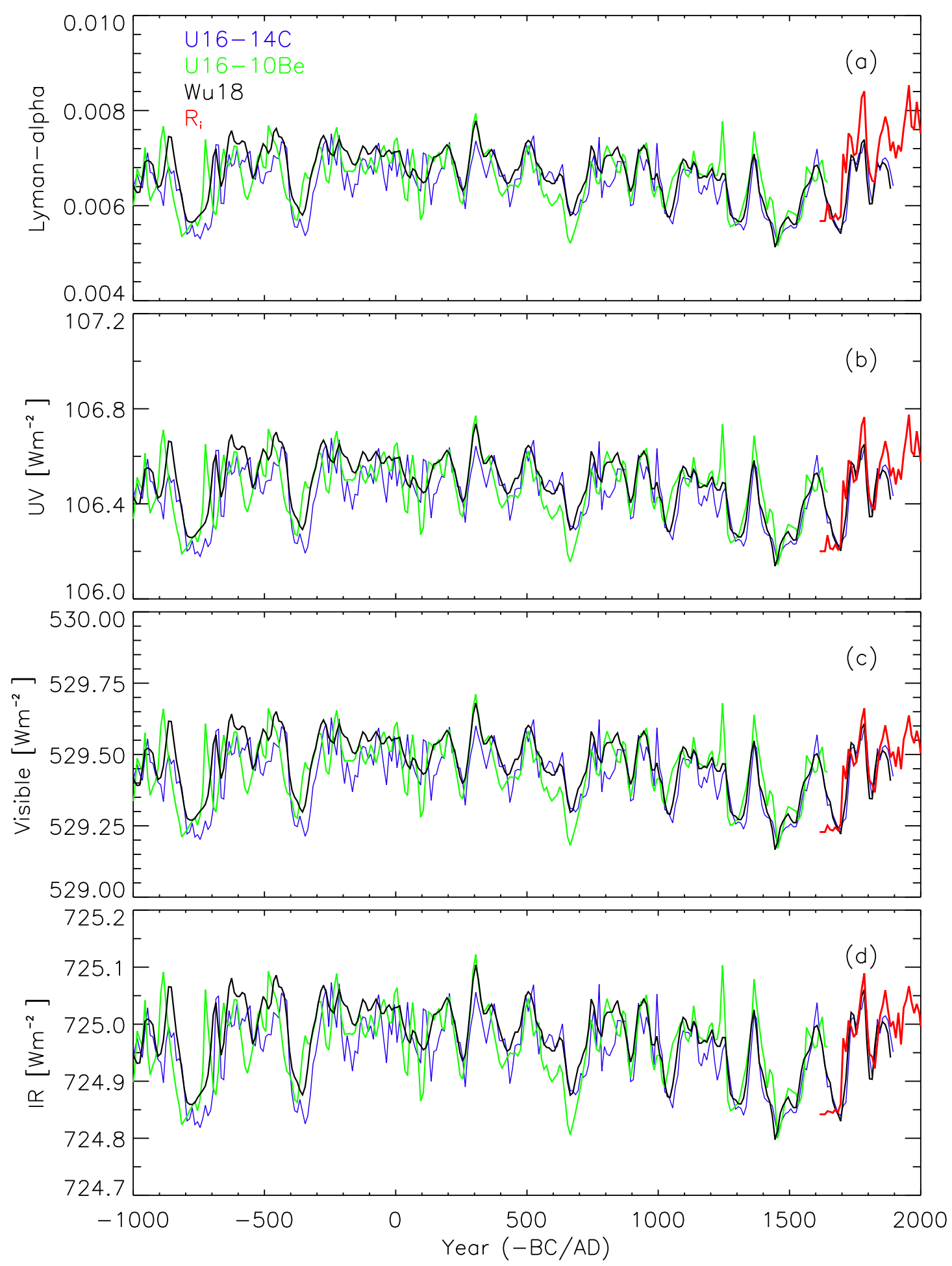

Figure 4.8: Reconstruction of UV/SSI integrated over a) Lyman- $\alpha$, b) $115-400 \mathrm{~nm}$ (marked UV on the axis label), c) $400-700 \mathrm{~nm}$ and d) wavelengths longward of $700 \mathrm{~nm}$ (IR) based on three isotope-based SN series (see legend in the plot) over period $1000 \mathrm{BC}$ $-2000 \mathrm{AD}$. The reconstruction based on the directly observed SN is shown in thick red line after 1639. All curves are decadal averages. 


\subsection{Summary}

Solar radiation is the dominant energy source to Earth's climate. In addition to the known quasi 11-year solar cycle, the Sun varies on longer time scales, and this variability might affect the Earth. Since direct solar irradiance measurements only exist after 1978, this record is too short for assessing the solar influence on climate, so that reconstructions of past irradiance changes are needed. Models assuming that irradiance variations on time scales longer than about a day are caused by the evolution of the solar surface magnetism reproduce over $90 \%$ of the measured irradiance variability (e.g., Krivova et al. 2003; Ermolli et al. 2003, 2013; Ball et al. 2014; Yeo et al. 2014b; Coddington et al. 2016). In particular, the newest version of SATIRE (Yeo et al. 2017a) does so without any recourse to the irradiance measurements (i.e., without free parameters). Thus here we assume that the same is also true on longer time scales up to millennia and reconstruct solar total and spectral irradiance over the last 9 millennia from proxies of solar magnetic activity. In particular, we use the SATIRE-T (Krivova et al. 2007, 2010) and -M (Vieira et al. 2011) models, in which the evolution of the solar surface magnetic field is reconstructed from the sunspot number (Clette et al. 2014; Clette and Lefévre 2016; Chatzistergos et al. 2017) and cosmogenic isotope data (Usoskin et al. 2016a; Wu et al. 2018b), respectively.

First, the SATIRE-T model has been re-visited with several modifications and updates. The changes include: modified ephemeral region cycle description (to assure the fact that the ER cycle maxima occur at or before the corresponding AR maxima), a more realistic description of the spatial distribution of faculae and sunpots (activity belts), updates in the input sunspot number series and the reference series (OMF, TMF, TSI, UV and facular contribution to the TSI variation). The reconstruction with the SATIRE-T is in close agreement with the observations over the last 4 decades. The modelled open magnetic flux also agrees well with the independent reconstruction based on the $a a$-index over the last 150 years (Lockwood et al. 2014c). The reconstructed TSI shows an increase between the late 17 th century and the present of $0.92_{-0.05}^{+0.15} \mathrm{~W} / \mathrm{m}^{2}$, which is somewhat lower (but within the mutual uncertainty ranges) than in the earlier reconstructions with SATIRET (Krivova et al. 2007, 2010), SATIRE-T2 (Dasi-Espuig et al. 2016), and the NRLTSI model (Coddington et al. 2016). This is significantly lower than in the reconstruction by Shapiro et al. (2010) and Egorova et al. (2018).

Keeping the free parameters fixed, we then used the SATIRE-M model and the SN series reconstructed from cosmogenic data, to calculate the TSI and SSI over the last 9000 years. Of the three isotope-based $\mathrm{SN}$ series, two are individual ${ }^{14} \mathrm{C}$ and ${ }^{10} \mathrm{Be}$ series from Usoskin et al. (2016a), and one in the newest multi-isotope composite by Wu et al. (2018b). The three TSI/SSI reconstructions with the SATIRE-M model show a mutually similar long-term variability. The range of the TSI variability on millennial time scales for the three used isotope series is about $0.11 \%\left(1.5 \mathrm{~W} / \mathrm{m}^{2}\right)$. After the Maunder minimum, the reconstruction from the cosmogenic isotopes is consistent with that from the direct SN observation. Furthermore, over the 19th century, the agreement with the reconstruction from the SN by Chatzistergos et al. (2017) is better than with that from the WDC-SILSO SN series (Clette et al. 2014).

This reconstruction is the first SSI reconstruction over the Holocene, which uses physics-based models to describe all involved processes (Vieira and Solanki 2010; Vieira et al. 2011), and also the first one based on a composite ${ }^{14} \mathrm{C}-{ }^{10} \mathrm{Be}$ record (Wu et al. 2018b), 
and is recommended for studies of long-term climate changes (e.g., Jungclaus et al. 2016). The TSI/SSI reconstructions are available at the webpage of MPS "Solar Variability and Climate" group 5 .

\footnotetext{
${ }^{5}$ http://www2.mps.mpg.de/projects/sun-climate/data/
} 


\title{
5 Postdicting Solar Cycles on Millennial Time Scale
}

\author{
To be submitted to A\&A by C.-J Wu ${ }^{1,2}$, N. A. Krivova ${ }^{1}$ and S. K. Solanki ${ }^{1,3}$.
}

\begin{abstract}
Contents. Reconstructions of the solar irradiance over millennia are usually done with a decadal resolution, yet the 11-year signal is important for paleo-climate models.

Aims. The aim of this work is to provide annually sampled records of sunspot numbers and irradiance, including solar cycle variations, over millennia using decadally-averaged cosmogenic isotope data as input.

Methods. We employ a regression analysis to obtain relationships that allow four key characteristics of the solar cycle seen in sunspot number to be derived from the decadallyaveraged sunspot number values. Furthermore, we introduce a new solar cycle shape function, which allows the shape to be described using only four known cycle characteristics without free parameters. The derived relationships are applied to obtain annually sampled sunspot numbers from three time series of cosmogenic isotope data including a new multi-isotope composite.
\end{abstract}

Results. The statistical approach introduced here is tested against the actually measured sunspot number. The solar cycle shape function is shown to reproduce the observed shapes of the solar cycles since 1700 rather well $\left(R_{\mathrm{c}}=0.96\right)$. The amplitudes of the reconstructed solar cycles also display good agreement with the observed cycle amplitudes $\left(R_{\mathrm{c}}=0.84\right)$. The phase of the cycles is obtained less stably and can drift with time compared with the directly observed phase. After validation, the new method is used to reconstruct a yearly sunspot number record spanning the last 9000 years. This in turn is employed to reconstruct the solar irradiance with the same cadence over the same period of time.

conclusions. We have presented a new method for reconstructing the solar cycle from decadally sampled cosmogenic isotopes. It represents the true solar cycle better than earlier attempts and allows providing yearly sampled and sunspot numbers and irradiance to climate modellers.

\footnotetext{
${ }^{1}$ Max-Planck-Institut für Sonnensystemforschung, Justus-von-Liebig-Weg 3, Göttingen, Germany

${ }^{2}$ Georg-August-Universität Göttingen, Institute for Astrophysics, Göttingen, Germany

${ }^{3}$ School of Space Research, Kyung Hee University, Yongin, Gyeonggi-Do,446-701, Republic of Korea
} 


\subsection{Introduction}

The Sun is our closest star and the dominant energy source to the Earth. The energy flux per unit area at the mean Sun-Earth distance is termed total solar irradiance (TSI). TSI varies at various time scales, from about $0.1 \%$ on the solar cycle time scale to about $0.3 \%$ on the solar rotation time scale (see reviews by Ermolli et al. 2013; Solanki et al. 2013). The assessment of the TSI variability is important since it has impact on the Earth's climate (e.g., Haigh 2007; Gray et al. 2010; Schmidt et al. 2011; Jungclaus et al. 2016; Matthes et al. 2017).

To better understand the influence of solar irradiance on Earth's climate system on various time scales, one needs long-term and continuous solar records. However, the direct regular observations of the solar irradiance are available only since 1978. Models, therefore, are required to reconstruct the solar irradiance back in time. There have been a number of models developed to reconstruct solar irradiance on centennial time scales using the sunspot observations as inputs (e.g., Solanki and Fligge 1999; Fligge and Solanki 2000; Lean 2000; Krivova et al. 2010; Shapiro et al. 2010; Dasi-Espuig et al. $2014,2016)$. Due to the lack of direct observations of solar activity, solar irradiance reconstruction on millennial time scales requires different proxies from sunspot number or sunspot area. Cosmogenic isotope concentrations in the terrestrial archives are considered as alternative indicators of solar activity since the cosmogenic isotopes are produced by galactic cosmic rays (GCRs), whose flux is modulated by the heliospheric and geomagnetic field (Beer 2000a; Usoskin et al. 2002). Earlier studies have reconstructed the TSI from the isotope data, using either simple linear regressions (Steinhilber et al. 2009, 2012; Delaygue and Bard 2011; Shapiro et al. 2011), or a physics-based model (Vieira et al. 2011; Wu et al. 2018a).

The solar irradiance reconstructions based on cosmogenic isotopes are often limited to data with a temporal resolution of about 10 years, which smooths out the information on the 11-year solar cycle (SC). However, in climate models, all energy forcing inputs (such as volcanic, vegetation, land use) should have consistent temporal resolution to avoid any biases in the climate simulations (Schmidt et al. 2011). Several attempts have been made to remedy the missing information on SCs in the irradiance reconstructions. For instance, Steinhilber et al. (2009) superimposed sinusoidal functions with a periodicity of 11-year on the recosntructed solar modulation potential $(\phi)$, while Schmidt et al. (2011) utilised a synthetic 11-year SC. Although these two methods provide a higher temporal resolution with cyclic variation, they are not realistic.

The motivation of this study is to simulate the SCs using a statistical and more realistic approach. We will first quantify the main SC properties using the directly measured sunspot number (SN) series. We will then search for a set of statistical relationships between these properties and the corresponding decadally-averaged SN. The derived relationships will be applied to three sets of cosmogenic isotope-based SN series. Two of these are based on individual cosmogenic isotope records $\left({ }^{14} \mathrm{C}\right.$ and ${ }^{10} \mathrm{Be}$, respectively), while the third one is a multi-isotope composite constructed by Wu et al. (2018b, Paper I hereafter). The simulated SN series with SCs has a 1-year temporal resolution and, will then be input into the SATIRE model, following Wu et al. (2018a, Paper II hereafter), to reconstruct the total and spectral solar irradiance over the last 9000 years with an annual cadence.

This paper is structured as follows. We will first introduce the three cosmogenic iso- 
tope data sets in Sect. 5.2. The statistical approach to simulate SCs will be described in Sect. 5.3. The reconstructed SN and TSI/SSI with solar cycles will be presented in Sect. 5.4. Finally, the results will be discussed and summarized in Sect. 5.5.

\subsection{Cosmogenic isotope records}

When GCRs interact with the Earth's upper atmosphere, a cascade of reactions takes place and a wide range of species of secondary particles and cosmogenic isotopes are produced. The flux of GCRs is modulated by both the heliospheric magnetic field and the geomagnetic field. Therefore, cosmogenic isotope abundances in natural archives, such as ${ }^{14} \mathrm{C}$ and ${ }^{10} \mathrm{Be}$ retrieved from tree rings and ice cores, respectively, are commonly seen as indicators of historical solar magnetic activity on millennial time scales, once other effects have been removed.

After being produced in the upper atmosphere, these two isotopes follow different geochemical paths in the Earth's climate system. ${ }^{14} \mathrm{C}$ is easily oxidized into ${ }^{14} \mathrm{CO}_{2}$ and takes part in the global carbon circulation until it settles in sediments or is absorbed by living organisms (Kovaltsov et al. 2012). The signal of ${ }^{14} \mathrm{C}$ is, therefore, globally wellmixed. In contrast, ${ }^{10} \mathrm{Be}$ usually attaches to aerosols, and precipitates within 1-2 years mainly through wet deposition into local water reservoirs or accumulates in the ice sheets. Hence, the signals in ${ }^{10} \mathrm{Be}$ series are highly subjected to the local climate conditions.

The conversion of the original measured signals of ${ }^{14} \mathrm{C}$ and ${ }^{10} \mathrm{Be}$ (typically in units of production rates or concentrations) into Sun-related quantities is not trivial, due to many non-linear processes involved in the isotope production and deposition. To account for this, physical models have been developed. First, isotope production models (Masarik and Beer 1999; Kovaltsov et al. 2012; Poluianov et al. 2016) and atmospheric transport/deposition models (Heikkilä et al. 2009; Roth and Joos 2013) are used. Next, geomagnetic field models (Yang et al. 2000; Korte and Constable 2005; Knudsen et al. 2008; Usoskin et al. 2016a) are employed to hive off the solar modulation potential, $\phi$, which is the first Sun-related quantity that can be determined from the cosmogenic data. It describes the energy loss of GCRs in the heliosphere due to the modulation by the solar magnetic field (e.g., Usoskin et al. 2005). It is, however, a relative quantity whose absolute level is model-dependent (Herbst et al. 2010, 2017; Usoskin et al. 2005) and is not suitable to be used directly as a proxy of solar magnetic activity.

Although some early studies reconstructed the solar irradiance (Steinhilber et al. 2009, 2012; Delaygue and Bard 2011; Shapiro et al. 2011), the linear regressions between $\phi$ and TSI that they used are not expected to capture all the non-linear physcis processes involved. Therefore, Usoskin et al. (2003, 2016a); Solanki et al. (2004) and Wu et al. (2018b) converted $\phi$ into a more definitive index, $\mathrm{SN}$, which then served as a proxy of solar activity used to reconstruct the solar irradiance using essentially a form of the SATIRE-T model adapted to the decadal sampling rate (Vieira et al. 2011; Wu et al. 2018a).

In this study, we use three $\mathrm{SN}$ series derived from cosmogenic isotope data, of which two series are based on individual cosmogenic isotopes $\left({ }^{14} \mathrm{C}\right.$ and ${ }^{10} \mathrm{Be}$, respectively), while the third one is the newest composite of seven isotopes records (paper I). The ${ }^{14} \mathrm{C}$-based SN series, "U16-14C", is reconstructed by Usoskin et al. (2016a) from the globallyaveraged ${ }^{14} \mathrm{C}$ production rate (Roth and Joos 2013) from the original $\Delta^{14} \mathrm{C}$ measurements 
in the tree rings (INTCAL09, Reimer et al. 2009) and adopts an updated geomagnetic model (GMAG.9k). The ${ }^{10} \mathrm{Be}$-based SN series, "U16-10Be", is reconstructed from the sample in the Greenland Ice Core Project (GRIP; Yiou et al. 1997; Muscheler et al. 2004; Vonmoos et al. 2006) with an updated ${ }^{10} \mathrm{Be}$ production rate model (Kovaltsov and Usoskin 2010 ) and the GMAG.9k geomagnetic model. Due to the common modulation origin (viz. the Sun), the signals of ${ }^{14} \mathrm{C}$ and ${ }^{10} \mathrm{Be}$ share a high degree of similarity while certain temporal discrepancies between them are likely due to the different geochemical processes in the atmosphere (Muscheler et al. 2014; Adolphi and Muscheler 2016; Wu et al. 2018b). In addition, the ${ }^{10} \mathrm{Be}$ signals lack an absolute level calibration due to the different assumptions in the snow accumulation and deposition for each individual drilled ice core. In the paper I, we have accounted for this and constructed a multi-isotope composite, "Wu18", using one global ${ }^{14} \mathrm{C}$ and six local ${ }^{10} \mathrm{Be}$ series. This time series takes into account uncertainties in the ${ }^{10} \mathrm{Be}$ absolute chronology by wiggle matching with respect to the ${ }^{14} \mathrm{C}$ time series. The information on these three data sets and their periods is given in Table 4.2. All three data sets have a decadal temporal resolution. The statistical approach to simulate the SCs from the decadally-averaged values will be introduced in the next section.

\subsection{Methodology}

Multiple studies have shown that the SC characteristics (e.g., the amplitude or the cycle length) vary from one cycle to another. A number of empirical relationships have also been found between various SC parameters (e.g., Waldmeier 1935, 1939; Dicke 1978; Wilson 1987; Hathaway et al. 2002; Solanki et al. 2002a). For instance, stonger cycles tend to rise faster than weaker ones (Waldmeier Effect, Waldmeier 1935, 1939). The cycle amplitude tends to be anti-correlated with the length of the previous cycle (AmplitudePeriod Effect, Wilson et al. 1998; Hathaway et al. 2002; Solanki et al. 2002a; Kane 2008). Wilson et al. (1998) has also found the Amplitude-Minimum Effect, namely that the cycle amplitude is correlated with the level of the preceding cycle minimum. We note that the "amplitude" in these earlier studies is usually defined as the maximum SN reached during a cycle (usually monthly smoothed or yearly smoothed), and is different from our definition below (Sect. 5.3.5).

Moreover, there is an indication that the SC characteristics to be related to the overall solar activity level. Thus Fligge et al. (1999) have shown that during the Maunder minimum, which was an extended period of generally very weak solar activity, solar cycles were noticeably longer than 11 years. Since the decadally-averaged SN reconstructed from the cosmogenic isotopes can be considered as a measure of the overall solar activity, the potential links, such as those mentioned above, might help us to connect the decadal $\mathrm{SN}$ values with the characteristics of individual cycles. Based on this concept, we developed a sequence of procedure to simulate the SC parameters from the decadally-averaged SN. Here, we first outline the three main steps of the procedure, while more details on each step are given in the following individual sections:

1. First, we do a statistical study of various SC parameters to establish the potential links between them and the decadal SN values. Then we simulate the SC parameters back in time from the decadal SN values using such relationships (Sect. 5.3.1). 
2. We introduce a new SC shape fit function, which is then used to refine the SC shape between the simulated cycle minimum and maxima (as well as between the cycle maxima and minima). This function requires only four simulated SC characteristics from the step (1) with no further free parameters being needed (Sect. 5.3.2).

3. We compare the result and the directly observed SN over the overlapping period. The phase of the simulated SN, which cannot be determined independently, is then be adjusted by maximizing the cross correlation coefficient (Sect. 5.3.3).

\subsubsection{Statistical approach}

To recover the information on the past SCs from the decadally-averaged SN, we first search for the potential relationships between various SC properties and the decadallyaveraged values using the directly observed SN.

Following the paper I, we use the updated version (v2.0) of the annual international sunspot number series by WDC-SILSO ${ }^{1}\left(\mathrm{~S}_{\mathrm{Nv} 2}\right.$, Clette et al. 2014). We also employ the group sunspot number series (GSN) constructed by Chatzistergos et al. (2017, $R_{\mathrm{CH}}$ hereafter) to estimate the uncertainty of the results (Sect. 5.4.1) due to the uncertainty in the $\mathrm{SN}$ record. $R_{\mathrm{CH}}$ is a new GSN series, which is based on a non-linear non-parametric method for cross-calibration of individual historical GSN records.

Using the directly observed SN we quantify four main SC properties: (1) annual SN at the cycle maximum, $S_{\max }$, (2) annual SN at the preceding minimum, $S_{\min }$, (3) cycle rise time, $L_{\text {rise }}$, and (4) cycle length, $L_{\text {cyc }}$. Together with the SC shape function (which will be introduced in Sect. 5.3.2), these give a relatively complete description of a cycle.

Earlier studies have compared various SC properties with each other (e.g., Waldmeier 1935, 1939; Hathaway et al. 2002; Solanki et al. 2002a; Karak and Choudhuri 2010). We here compare each property directly with the decadally-averaged SN, $\langle\mathrm{SN}\rangle$. Moreover, even though a potential correlation between $S_{\max }$ and $S_{\min }$ has been suggested (Wilson et al. 1998), we compare these two characteristics with the $\langle\mathrm{SN}\rangle$ individually. This is because the $\langle\mathrm{SN}\rangle$ is the only information available from the isotope data, and thus determining one parameter from another could easily propagate the errors.

The levels of both the SN maxima and minima affect the decadal SN average. Therefore, we compare the $\langle\mathrm{SN}\rangle$ with two parameters, $S_{\max }$ and $S_{\min }$ that are closest to it. Then we compare the $\langle\mathrm{SN}\rangle$ with the $L_{\text {rise }}$ of the cycle $(k)$ who possesses that found $S_{\max }$. However, due to the anti-correlation between the strength of a given cycle and the length of the previous cycle, we compare the $\langle\mathrm{SN}\rangle$ with the length of the previous cycle $\left(L_{\mathrm{cyc}, k-1}\right)$.

Figure 5.1 visualizes how the decadally-averaged $\mathrm{SN}$ values are compared with the SC parameters. The annual $\mathrm{S}_{\mathrm{Nv} 2}$ series over the period 1700-2017 is shown in panel a, where the numbers of the cycles (C) are indicated above the curve. The SN series is segmented into calendar decades beginning from 1700, as shown by the vertical dashed lines, where the numbers of the decades (D) are denoted in the upper X-axis. The decadally-averaged $\mathrm{SN},\left\langle\mathrm{S}_{\mathrm{Nv} 2}\right\rangle$, is shown by a dot in each decadal segment. Since the solar cycle lengths are not exact 10 years, there are in total 32 decades corresponding to 29 cycles. For instance, the decadal values of both D4 and D5 are compared with the $S_{\max }$ and $L_{\text {rise }}$ of cycle C0, and the $L_{\text {cyc }}$ of cycle C- 1 .

\footnotetext{
${ }^{1}$ http://www. sidc.be/silso/datafiles
} 


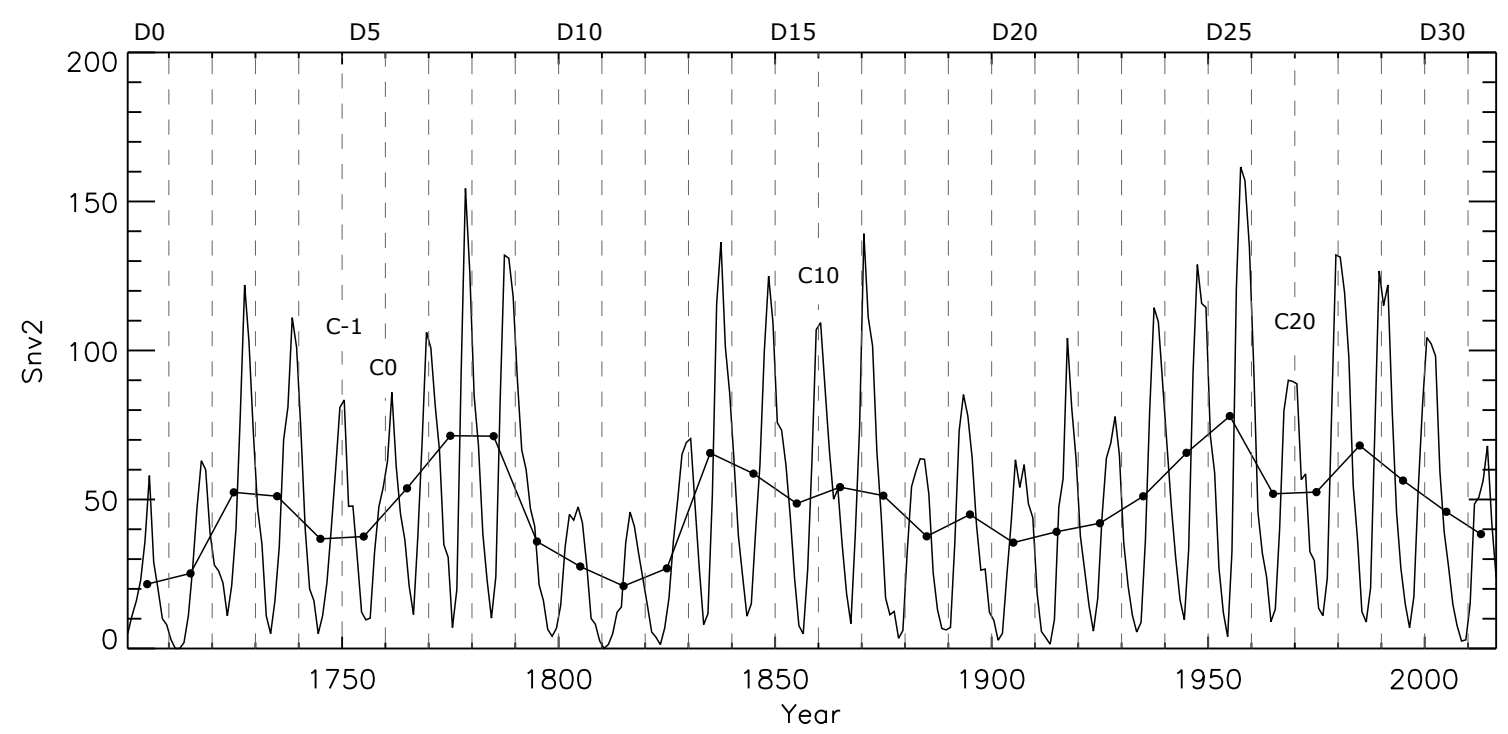

Figure 5.1: Observed $\mathrm{SN}$ series, $\mathrm{S}_{\mathrm{Nv} 2}$, segmented into calendar decades (vertical dashed lines). The decadal $\mathrm{SN}$ value, $\left\langle\mathrm{S}_{\mathrm{Nv} 2}\right\rangle$, calculated in each decade is shown by a dot. The numbering of the decadal segments is denoted as $\mathrm{D}$ in the upper $\mathrm{x}$-axis, and the numbering of solar cycle as C. Note that in Sect. 5.3.5 we will test different segmentation dates and their influence on the results.

Figure 5.2 shows the relationships between the four SC parameters and the decadallyaveraged $\mathrm{SN},\left\langle\mathrm{S}_{\mathrm{Nv} 2}\right\rangle$. The solid lines represent the best fits in four panels, and the 3- $\sigma$ uncertainties are shown by the dashed lines. Note that since both, $\left\langle\mathrm{S}_{\mathrm{Nv} 2}\right\rangle$ and the SC parameters contain uncertainties, a commonly-used ordinary linear squares regression (OLS) is not suitable for finding the best fit, as it only takes the uncertainties in one variable into account. We hence apply the ordinary least squares bisector regression (OLS-bisector), which is defined as the fit that bisects the angles of two OLS regressions (i.e., standard OLS and inverse OLS, Babu and Feigelson 1992). By doing so, the best fit accounts for the uncertainties in both, independent and dependent variables. The Pearson correlation coefficient, $R_{\mathrm{c}}$, between the $\left\langle\mathrm{S}_{\mathrm{Nv} 2}\right\rangle$ and the $S_{\max }$ (panel a) is 0.86 , whereas the $R_{\mathrm{c}}$ between the $\left\langle\mathrm{S}_{\mathrm{Nv} 2}\right\rangle$ and the other three characteristic values (panel $\mathrm{b}-\mathrm{d}$ ) are $0.61,-0.66$, and -0.61 , respectively. The $R_{\mathrm{c}}$ values are also given in the corresponding panels.

We note that the relationships obtained so far are based on the decadal SN values averaging from 1700, namely: $1700-1709,1710-1719, \ldots$, and so on. Later in Sect. 5.3.5, we will shift these decadal segments by $1-9$ years to examine the sensitivity of these linear relationships to the segmentation periods.

Using the set of relationships obtained above, we are now able to simulate (postdict) the SC parameters back in time from the $\left\langle\mathrm{S}_{\mathrm{Nv} 2}\right\rangle$. Nonetheless, based on our simulation approach, we can only determine the length of the previous cycle, whereas the length and the end timing of the last cycle of the series are unknown. These two values, $L_{\mathrm{cyc}, f}$ and $T_{\mathrm{end}, f}$, are, hence, needed as initial conditions in this approach. According to the current decaying SC, we take 2019 as the end of the C24, whose cycle length would then be 11 years. 

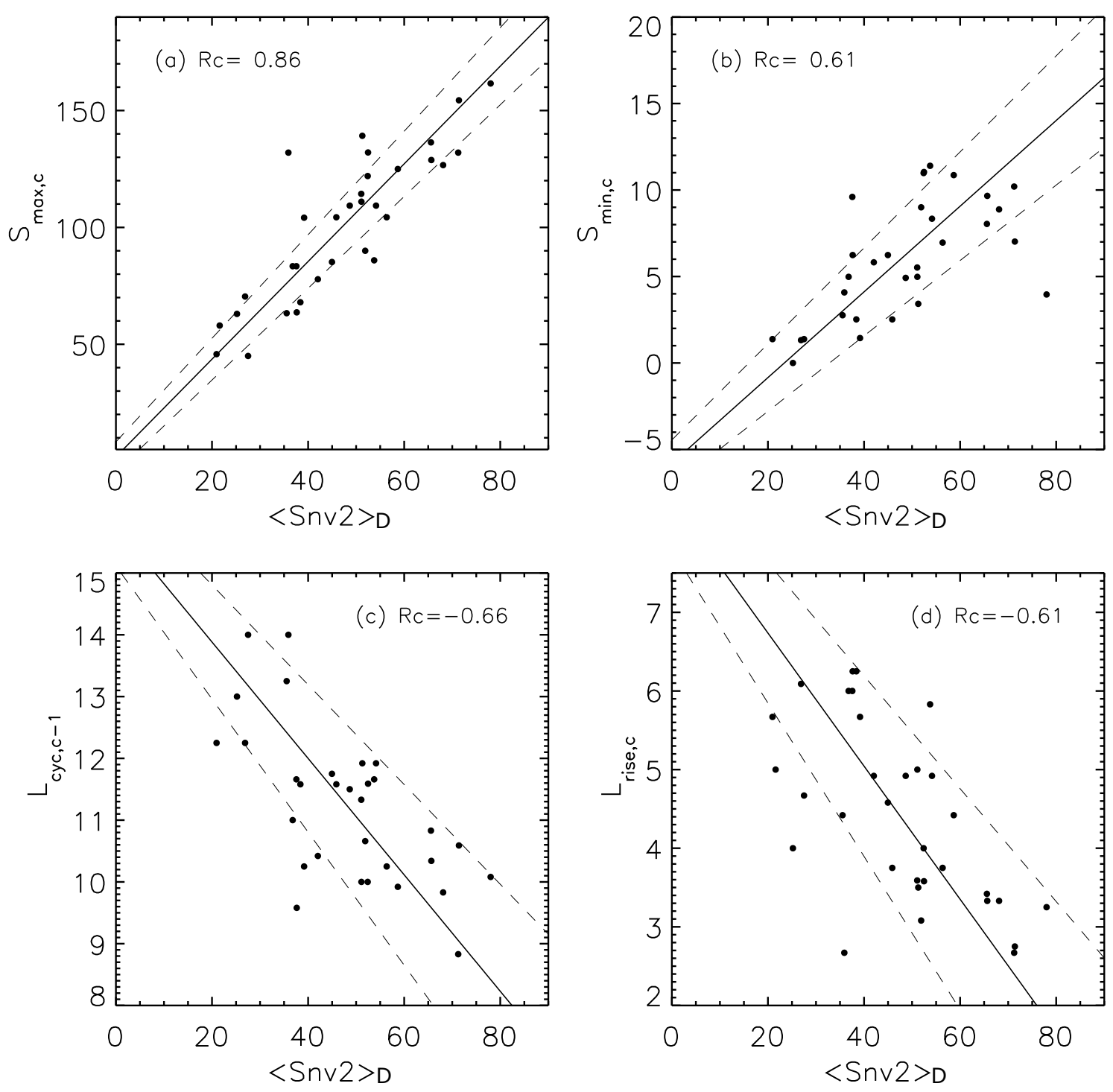

Figure 5.2: Four relationships of four studied SC parameters vs. $\left\langle\mathrm{S}_{\mathrm{Nv} 2}\right\rangle:$ (a) $S_{\max }$. (b) $S_{\min }$. (c) $L_{\text {cyc }}$. (d) $L_{\text {rise. }}$ The solid lines are the best OLS-bisector fits, with 3- $\sigma$ uncertainties shown by the dashed lines. Each data point represents one decade (D), the values are obtained by comparing with the SC parameters of the corresponding cycle (C), see the text for the details. 


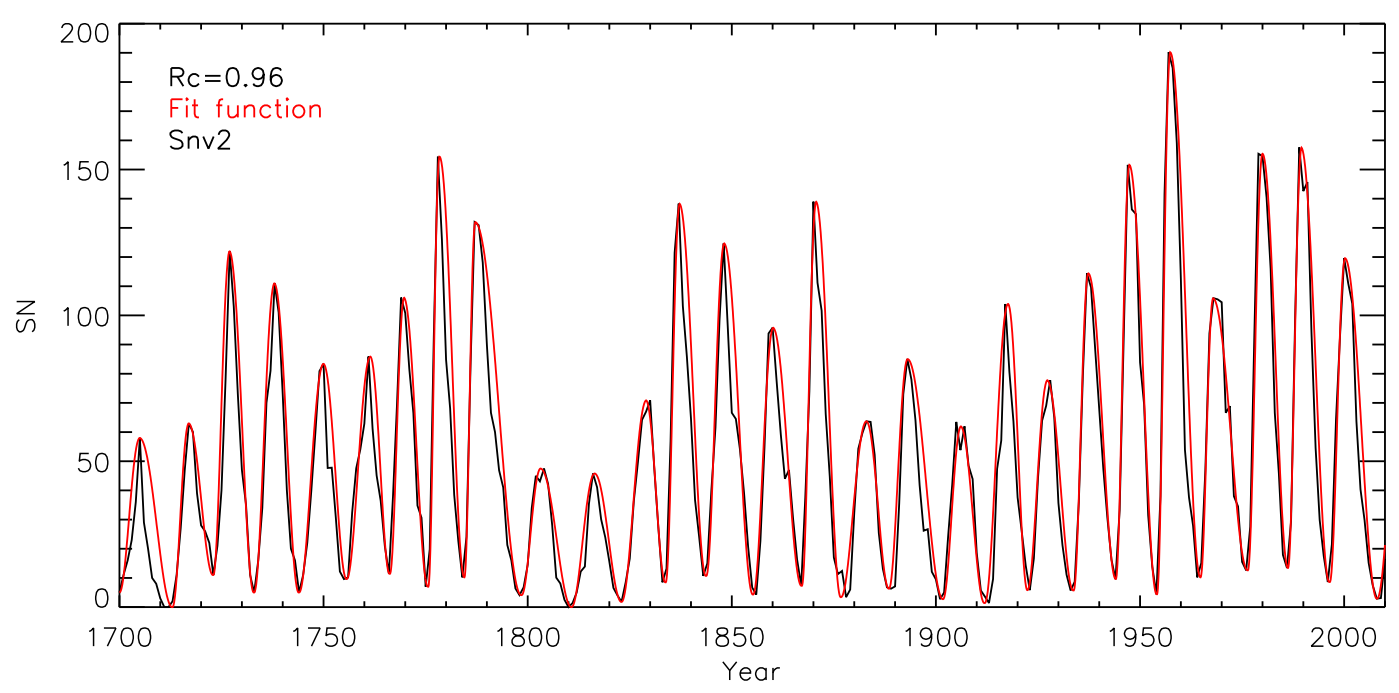

Figure 5.3: Comparisons of fitted SC shape (red) and directly measured SN, $\mathrm{S}_{\mathrm{Nv} 2}$ (annual, black) after 1700 with Pearson correlation coefficient $R_{\mathrm{c}}=0.96$.

\subsubsection{Shape of solar cycle}

Following the step (1) of the procedure, we can only recover the timings of the $S_{\max }$ and $S_{\min }$ in the past, without any information on the shape of the cycle. Therefore, the SC shape has to be determined and added separately. Multiple studies (e.g., Stewart and Panofsky 1938; Nordemann 1992; Elling and Schwentek 1992; Hathaway et al. 1994; Volobuev 2009) have dealt with the SC shape using simple functions with a number of free parameters, which varied from one cycle to another to fit the observed SC shape. All these proposed functions are, unfortunately, not directly suitable on millennial time scales as there is no information on the SC shapes in the past. Therefore, we need to search for a more general function to describe the SC shape without any free parameter.

Our method is inspired by Volobuev (2009), who proposed the first empirical fit function with only one free parameter. We re-derived this one-parameter function and optimized it into an empirical non-parametric fit function, $\mathcal{F}(t)$, with the four SC parameters obtained from the previous step (Sect. 5.3.1):

$\mathcal{F}(t)= \begin{cases}\frac{\left(S_{\max }-S_{\min 1}\right)}{\left(T_{\max }-T_{\min 1}\right)^{2}} \exp \left[1-\left(\frac{t-T_{\min 1}}{T_{\max }-T_{\min 1}}\right)^{2}\right]\left(t-T_{\min 1}\right)^{2}+S_{\min 1}, & T_{\min 1 \leq t \leq T_{\max },}, \\ \frac{\left(S_{\max }-S_{\min 2}\right)}{\left(T_{\min 2}-T_{\max }\right)^{2}} \exp \left[1-\left(\frac{t-T_{\min 2}}{T_{\min 2}-T_{\max }}\right)^{2}\right]\left(t-T_{\min 2}\right)^{2}+S_{\min 2}, & T_{\max } \leq t \leq T_{\min 2},\end{cases}$

where the subscripts 1 and 2 indicate the preceding and following cycle minimum, respectively. The rising phase of a SC is described by the top equation and the decaying phase by the bottom equation.

This function is validated on the known SC characteristics of the last 29 cycles after 
1700. The timings of the cycle maxima and minima are taken from the NOAA ${ }^{2}$ table. Figure 5.3 shows the comparison between the reconstructed cycles (red) and the observed annual $\mathrm{S}_{\mathrm{Nv} 2}$ (black). The correlation coefficient, $R_{\mathrm{c}}$, between the two series is 0.96 . It is gratifying that this non-parametric function, which bases only on four given cycle parameters, reproduces the SC shapes so well for such a large number of cycles with rather different properties. It is therefore a reasonable function for describing the SC shape of the simulated cycles occurring prior to the availability of direct sunspot observations.

\subsubsection{Phase adjustment}

The simulated SN series with realistic SC shapes from the previous two steps is now compared with the annual $S_{\mathrm{Nv} 2}$ over the overlapping period (if there is). Using cross correlation between the two series, a lag value of the simulated series can be determined. This value is then applied to the whole simulated series. However, one should keep in mind that this step can only try to adjust the phase for the last period that overlaps the $\mathrm{S}_{\mathrm{Nv} 2}$. And it does not guarantee the cycle phase of the whole time series would be fully and correctly adjusted, as shown in the examples in the next two sections.

This shortcoming is, unfortunately, inevitable. This is because unlike the uncertainties in the $S_{\max }$ and $S_{\min }$, which are independent from one cycle to the next, the uncertainty in the $L_{\text {cyc }}$ accumulates with time so that cycles can sometimes get out of phase. This becomes a problem especially when grand minima are encountered, as we do not know if the memory of the cycle phase is maintained across a grand minimum or not. The strength and length of the solar cycle of grand minima are not clear (e.g., Ribes and Nesme-Ribes 1993; Usoskin et al. 2000; Mursula et al. 2001; Vaquero et al. 2015; Zolotova and Ponyavin 2015; Svalgaard and Schatten 2016).

\subsubsection{Test simulation of the procedure}

We now examine if the whole procedure works under a general condition. Using the relationships obtained in Sect. 5.3.1 based on the $\left\langle\mathrm{S}_{\mathrm{Nv} 2}\right\rangle$ averaged over every consecutive 10 years from $1700 \mathrm{on}$, and the values of $\left\langle\mathrm{S}_{\mathrm{Nv} 2}\right\rangle$ as input, we simulate the SC properties and the shape following steps (1) and (2), respectively. Next, this simulated SN series is adjusted in phase compared with the annual $S_{\mathrm{Nv} 2}$ following the step (3), where a lag of 5.1 years is obtained. This value of the lag is then applied for the whole simulated period (in this case is $1700-2019$ ). The result (red) and the $S_{\mathrm{Nv} 2}$ (black) are shown in Fig. 5.4a. The correlation coefficient, $R_{\mathrm{c}}$, with a 30-year running window is shown in the panel $\mathrm{b}$. The frequency of the $R_{\mathrm{c}}$ is presented as a histogram in the panel c.

It can be seen from the Fig. 5.4a that the overall simulated amplitude is in a good agreement with the observed SN. However, Figs. 5.4a and 5.4b show that the phase of the cycles is only recovered well over the period $1790-1920$ with an average $R_{\mathrm{c}} \approx 0.9$, while the simulated series is out of phase by multiple years before 1750 and around 1940 with the correlation coefficient actually becoming negative at some times. Therefore, an overall correlation coefficient is 0.52 .

\footnotetext{
${ }^{2}$ https://www.ngdc.noaa.gov/stp/space-weather/solar-data/solar-indices/ sunspot-numbers/cycle-data/
} 


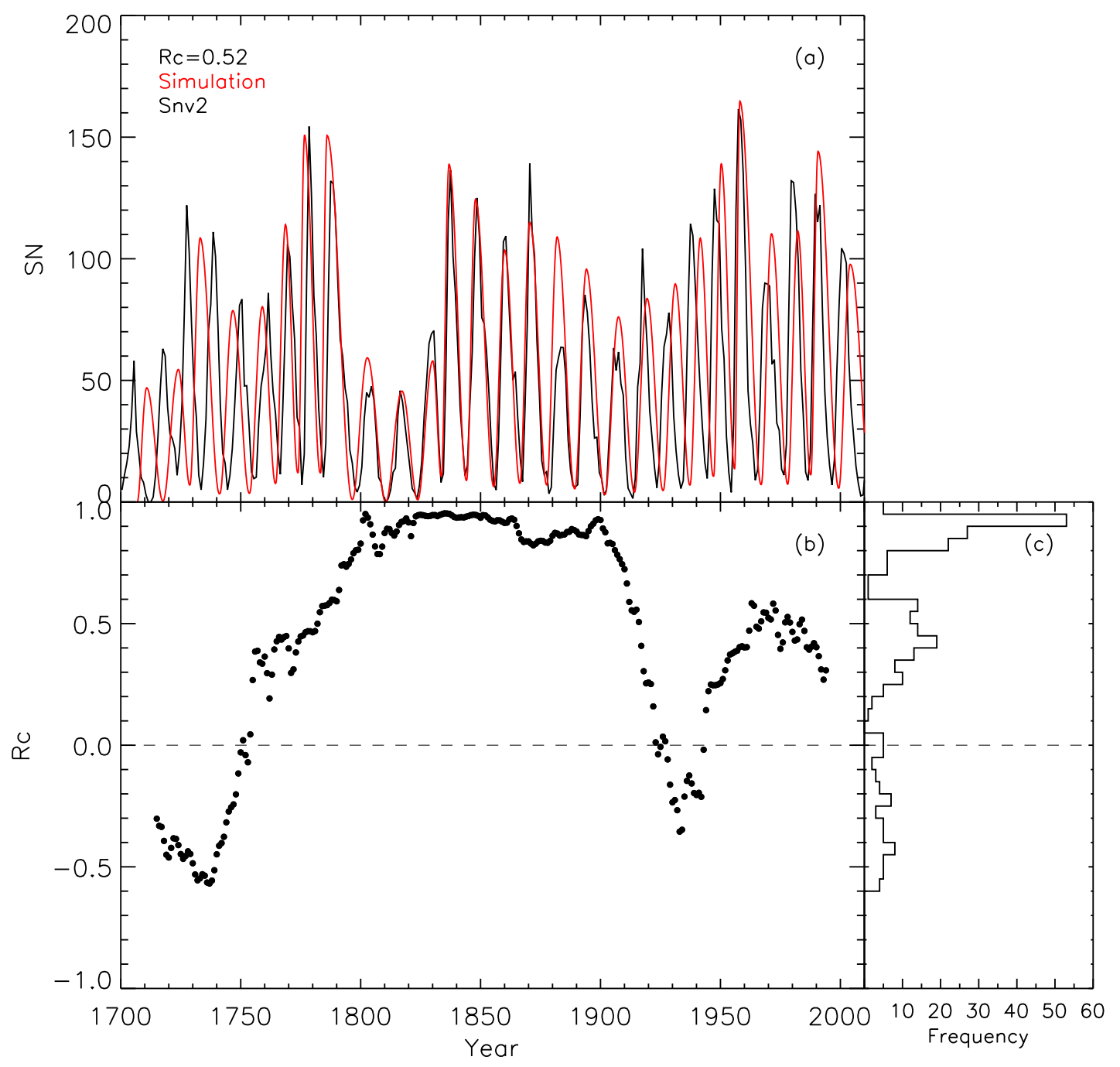

Figure 5.4: (a) Reconstructed from the decadally averaged observed $\mathrm{SN},\left\langle\mathrm{S}_{\mathrm{Nv} 2}\right\rangle$ (red), overplotted on the observed $\mathrm{S}_{\mathrm{Nv} 2}$ (black). (b) Running correlation coefficients, $R_{\mathrm{c}}$, determined over a running 30-year window centred at each year. (c) Frequency of $R_{\mathrm{c}}$ in (b). The dashed line indicates $R_{\mathrm{c}}=0$. 


\subsubsection{Sensitivity of the result to the decadal segmentation}

As described in Sect 5.3.1, $\left\langle\mathrm{S}_{\mathrm{Nv} 2}\right\rangle$ was calculated by averaging annual $\mathrm{S}_{\mathrm{Nv} 2}$ over every consecutive 10 years starting from 1700 (i.e., over the periods 1700-1709, 1710-1719, ... and so on), with a 0-year lag from 1700. Also, Sect. 5.3.4 uses these $\left\langle\mathrm{S}_{\mathrm{Nv} 2}\right\rangle$ as decadal inputs and the four linear relationships obtained therefrom, to simulate the four SC parameters. In reality, however, the cosmogenic isotope signals are sampled over any arbitrary ten years. Therefore, we now test how sensitive our results are to the choice of the decadal intervals, over which the averaging is done.

We now repeat the procedure as in Sect 5.3.1, but this time starting with the averaging one year later (i.e., 1701-1710, 1711-1720, ... and so on). This procedure is repeated 10 times until we have a total of 10 series, with the first 10-year averaging period of the 10th series being 1709-1718. These 10 decadally-averaged $\mathrm{SN}$ series are denoted as $\mathrm{H}_{i}$, where the subscript $i$ indicates the lag $0-9$ in years.

The values in each $\mathrm{H}_{i}$ series are then compared with the four SC parameters, same procedure as described in Sect. 5.3.1 and shown in Fig. 5.2. As a result, we obtain 10 sets of linear relationships with 10 lags. A set of four linear relationships is noted as $\mathrm{K}_{j}$, where the subscript $j$ indicates the lag $0-9$ in years. The correlation coefficients, $R_{\mathrm{c}}$, of the four SC parameters a functions of lag $(j)$ are shown in four panels in Fig. 5.5. It can be seen that all the correlation coefficients are rather stable.

Finally, since it is not clear what the correct lag is for a given data set (i.e., which correct $\mathrm{K}_{j}$ to use), we also need to test the $\mathrm{K}_{j}$ with the $\left\langle\mathrm{S}_{\mathrm{Nv} 2}\right\rangle$ with ten possible lags $\left(\mathrm{H}_{i}\right)$. Therefore, there are in total hundred possible combinations $\left(\mathrm{H}_{i=0 \ldots .}, \mathrm{K}_{j=0 \ldots .}\right)$ to simulate the SC parameters and we examine the stability of all these 100 combinations.

Following the whole simulation procedure and these 100 combinations, we simulate $100 \mathrm{SN}$ series with annual cadence, which are compared to the $S_{\mathrm{Nv} 2}$. Figure 5.6 shows the correlation coefficients between these 100 simulations and the $S_{\mathrm{Nv} 2}$ over the whole period of time $(1700-2017)$. The $\mathrm{x}$-axis represents the number of the $\left(\mathrm{H}_{i}, \mathrm{~K}_{j}\right)$ combinations in a two-digit format. For instance, 63 stands for the combination $\left(\mathrm{H}_{6}, \mathrm{~K}_{3}\right)$. The mean $R_{\mathrm{c}}$ value of these 100 simulated results is 0.59 . This relatively low correlation is attributed to the difficulty of recovering the precise SC phases over the whole period.

Since the overall solar activity, the amplitude of the solar cycle variation, and the annual temporal resolution are the most important ingredients for climate models, a few years of offset in the phase is not fatal. Hence, instead of using correlation coefficient of the whole time series, we use another quantity, 'amplitude', which is defined as the difference between the SN maximum and minimum of each cycle $(\Delta S)$. We compare the $\Delta S$ of the simulated SCs with that of the closest observed SC. The correlation coefficients of $\Delta S$ of the hundred combinations are shown in Fig. 5.7. The x-axis represents the $\left(\mathrm{H}_{i}, \mathrm{~K}_{j}\right)$ combination and the mean $R_{\mathrm{c}, \Delta S}$ value is 0.84 . Again, this results indicate that the SC can be simulated stably well regardless of the unknown lags $i$ and $j$.

Therefore, for the robustness, we combine all the data points of the four linear relationships from each 1-year lag test, as shown in Fig. 5.8, and take the sets of regression coefficients to simulate the SCs parameters from the $\left\langle\mathrm{S}_{\mathrm{Nv} 2}\right\rangle$ values: 

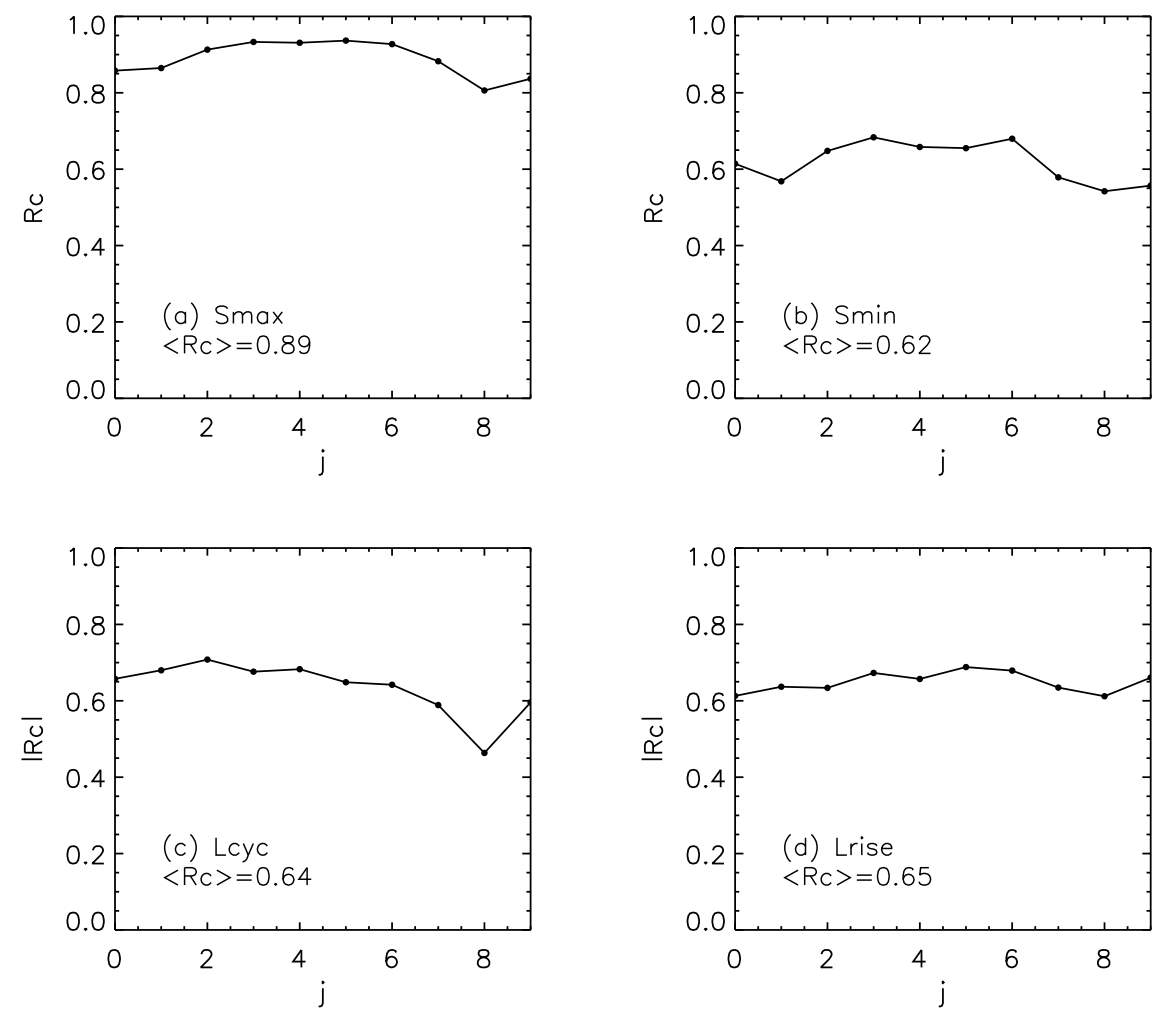

Figure 5.5: Pearson correlation coefficients, $R_{\mathrm{c}}$, between four SC parameters: (a) $S_{\max }$, (b) $S_{\text {min }}$, (c) $L_{\text {cyc }}$, (d) $L_{\text {rise }}$, and the $\left\langle\mathrm{S}_{\mathrm{Nv} 2}\right\rangle$. The x-axis indicates the lag $j$ in years from 1700. The $R_{\mathrm{c}}$ averaged over all 10 values of $j$ is given in each panel.

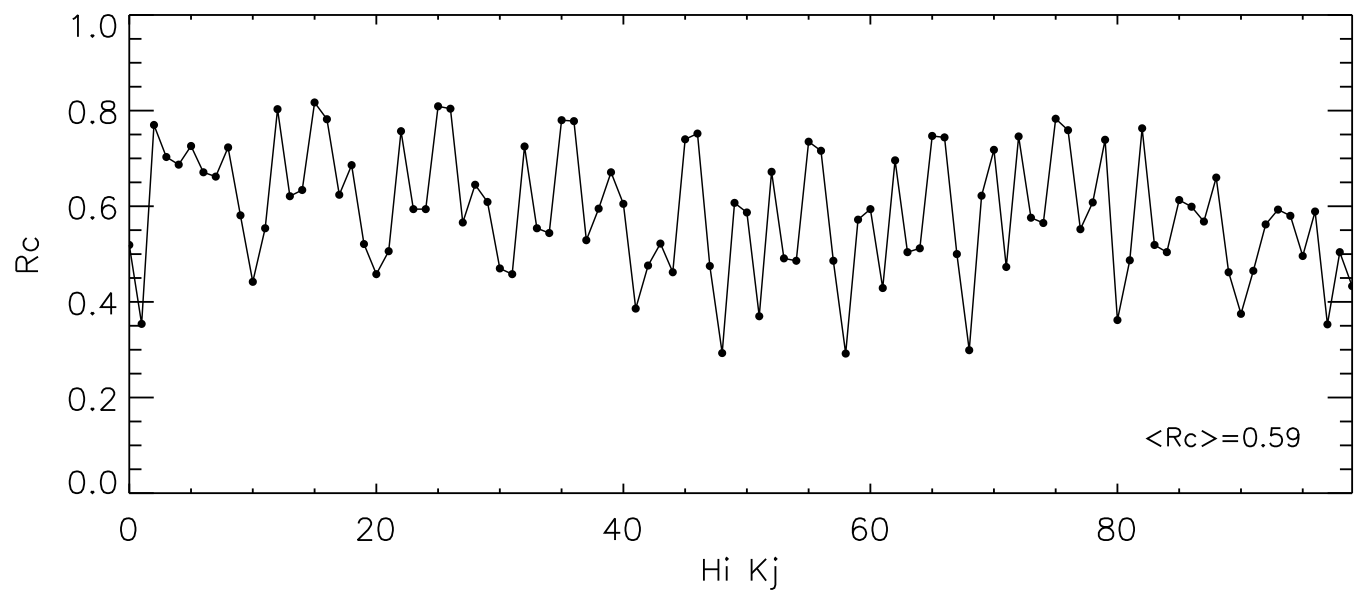

Figure 5.6: Pearson correlation coefficients between the reconstructed $\mathrm{SC}$ and the $\mathrm{S}_{\mathrm{Nv} 2}$. The $\mathrm{x}$-axis represents the 100 combinations $\left(\mathrm{H}_{i}, \mathrm{~K}_{j}\right)$ from the ten relationships and decadally-averaged SN with offsets $(j)$. 


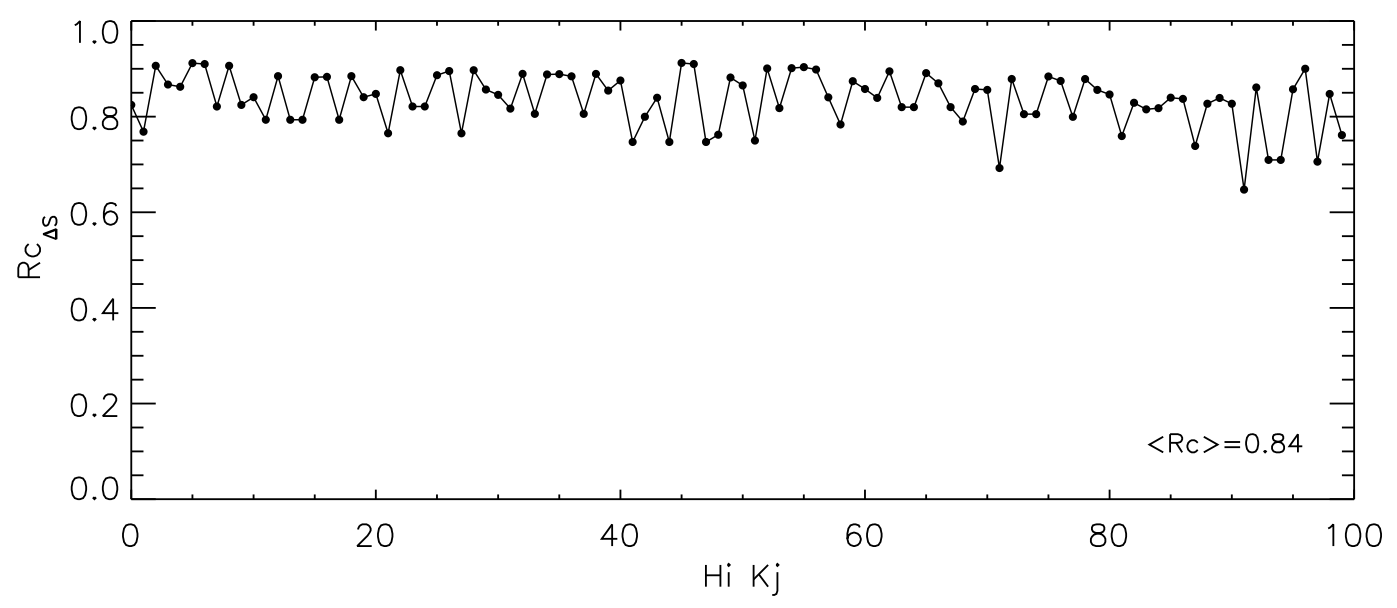

Figure 5.7: Correlation coefficients of $\Delta S\left(S_{\max }-S_{\min }\right)$ between the reconstructed SC and the measured $\mathrm{S}_{\mathrm{Nv} 2}$. The $\mathrm{x}$-axis represents the 100 combinations, $\left(\mathrm{H}_{i}, \mathrm{~K}_{j}\right)$, from the linear relationships and decadally-averaged $\mathrm{SN}$ with offsets $(j)$.

$$
\begin{gathered}
L_{\mathrm{rise}, \mathrm{c}}=-(0.07 \pm 0.01) \times\left\langle\mathrm{S}_{\mathrm{Nv} 2}\right\rangle_{\mathrm{D}}+(7.7 \pm 0.5) \\
L_{\mathrm{cyc}, \mathrm{c}-1}=-(0.08 \pm 0.01) \times\left\langle\mathrm{S}_{\mathrm{Nv} 2}\right\rangle_{\mathrm{D}}+(15.1 \pm 0.6) \\
S_{\mathrm{max}, \mathrm{c}}=(1.89 \pm 0.13) \times\left\langle\mathrm{S}_{\mathrm{Nv} 2}\right\rangle_{\mathrm{D}}+(8.6 \pm 6.9) \\
S_{\text {min,c }}=(0.22 \pm 0.03) \times\left\langle\mathrm{S}_{\mathrm{Nv} 2}\right\rangle_{\mathrm{D}}-(4.1 \pm 1.2)
\end{gathered}
$$

The SC simulation based on the $\left\langle\mathrm{S}_{\mathrm{Nv} 2}\right\rangle$ and this set of regression coefficients (Eqs. 5.2 $-5.5)$ are shown in Fig. 5.9. It can be seen that both the overall comparison $\left(R_{\mathrm{c}}=0.7\right)$ and the overall amplitude $\left(R_{\mathrm{c}, \Delta \mathrm{S}}=0.91\right)$ are in good agreement with the observed $\mathrm{S}_{\mathrm{Nv} 2}$, and are also significant better than those of the average of 100 combinations (Figs. 5.6 and 5.7).

Furthermore, as mentioned in Sect. 5.3.1 that we take the GSN series constructed by Chatzistergos et al. (2017), $R_{\mathrm{CH}}$, to estimate the uncertainty of the results. Therefore, we also do the same statistical study (not shown) based on the $R_{\mathrm{CH}}$ series. The set of four relationships obtained based on $\left\langle R_{\mathrm{CH}}\right\rangle$ series considering all 101 -year lags are:

$$
\begin{gathered}
L_{\text {rise }, \mathrm{c}}=-(0.08 \pm 0.01) \times\left\langle R_{\mathrm{CH}}\right\rangle_{\mathrm{D}}+(8.6 \pm 0.67) \\
L_{\mathrm{cyc}, \mathrm{c}-1}=-(0.09 \pm 0.02) \times\left\langle R_{\mathrm{CH}}\right\rangle_{\mathrm{D}}+(16.0 \pm 0.9) \\
S_{\text {max }, \mathrm{c}}=(1.8 \pm 0.2) \times\left\langle R_{\mathrm{CH}}\right\rangle_{\mathrm{D}}+(1.5 \pm 4) \\
S_{\text {min,c }}=(0.56 \pm 0.06) \times\left\langle R_{\mathrm{CH}}\right\rangle_{\mathrm{D}}-(15.7 \pm 3.5)
\end{gathered}
$$

The SC reconstruction based on the $\left\langle R_{\mathrm{CH}}\right\rangle$ and the relationships Eqs. (5.6) - (5.9) are present in Fig. 5.10. The regression coefficients obtained based on $R_{\mathrm{CH}}$ series are largely close to that based on $S_{\mathrm{Nv} 2}$ series, besides the coefficients in the simulating $S_{\text {min,c }}$. This is mainly attributed to the issue discussed in (Chatzistergos et al. 2017) that they might have overestimated the level of solar minima, and therefore the slope obtained here is higher than that obtained from $\mathrm{S}_{\mathrm{Nv} 2}$ series. In general, the two sets of relationships based on both, 

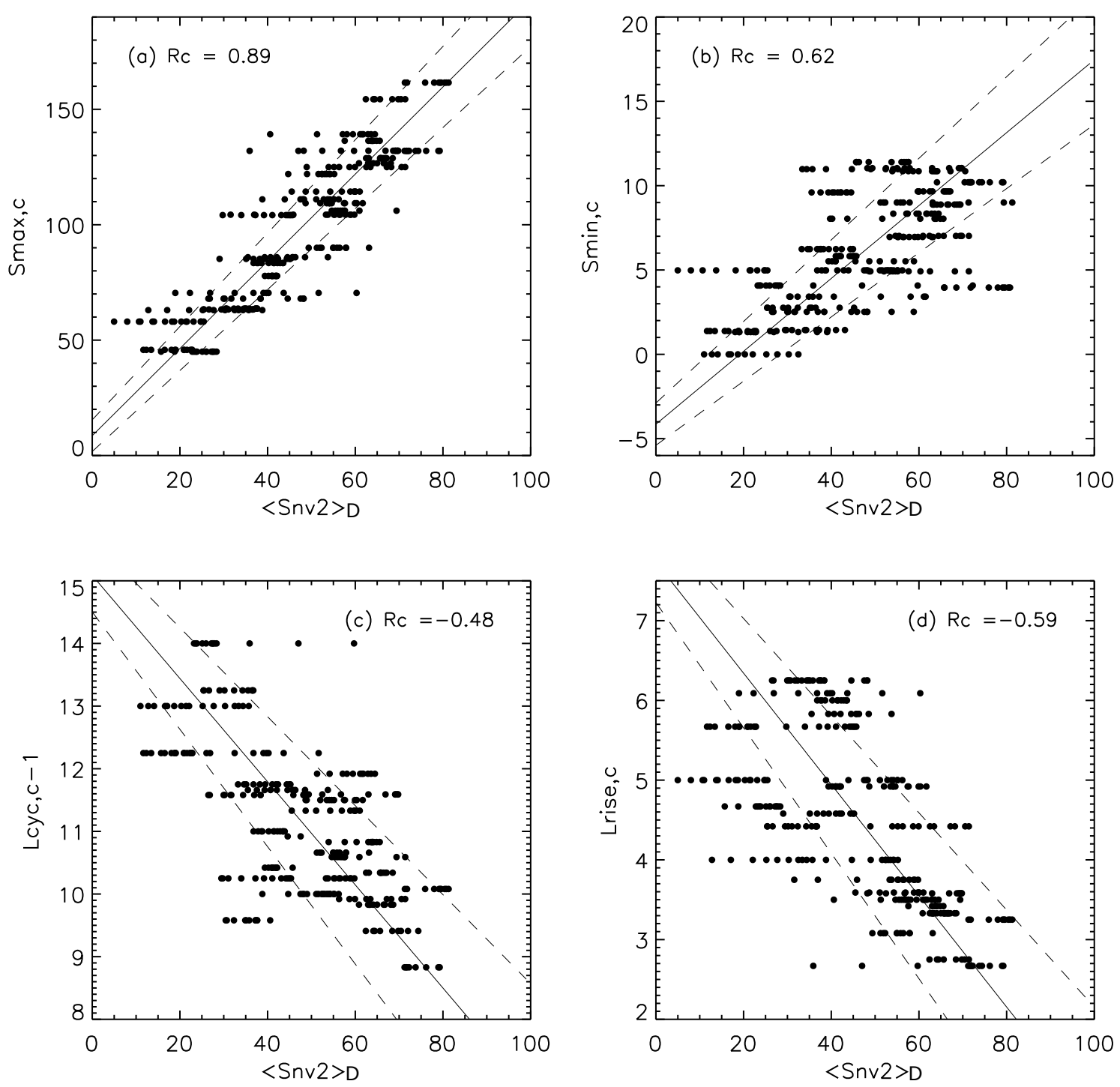

Figure 5.8: Same as Fig. 5.2, but this time considering all the data points from the relationships obtained with 10 1-year lag tests: (a) $S_{\max }$. (b) $S_{\text {min }}$. (c) $L_{\text {cyc }}$. (d) $L_{\text {rise }}$. The solid lines are the best OLS-bisector fits with 3- $\sigma$ uncertainty shown by the dashed lines. 


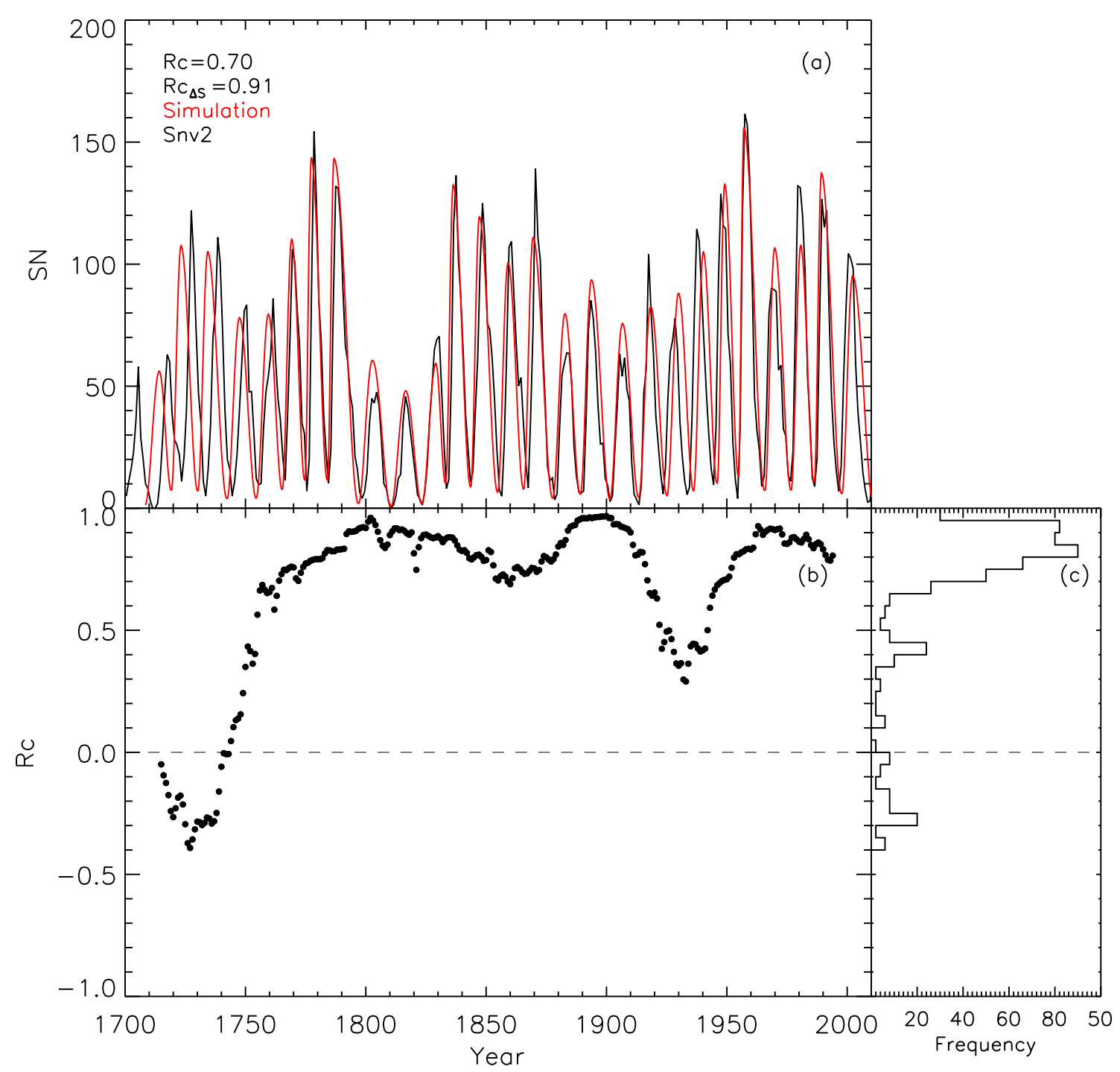

Figure 5.9: Same as Fig. 5.4, but reconstructed based on the final relationships Eqs. (5.2) $-(5.5)$ from the $\left\langle\mathrm{S}_{\mathrm{Nv} 2}\right\rangle$ series, $\mathrm{H}_{0}$.

$\mathrm{S}_{\mathrm{Nv} 2}$ and $R_{\mathrm{CH}}$ series, are close enough (within each other error range) and the simulation results show that this statistical approach is rather robust for simulating the SCs on longer time scales.

\subsection{Results}

\subsubsection{Simulation of solar cycles over the last 9 millennia}

We now use the approach described in Sect. 5.3 to simulated the SCs over the past 9000 years for the three sets of decadally-averaged $\mathrm{SN}$ derived from the cosmogenic isotopes (Sect. 5.2).

As shown in Sect. 5.3.1, we need two input quantities for this: the cycle length, $L_{\mathrm{cyc}, f}$, and the end time, $T_{\mathrm{end}, f}$, of the last cycle of the series. Two of three isotope-based SN 


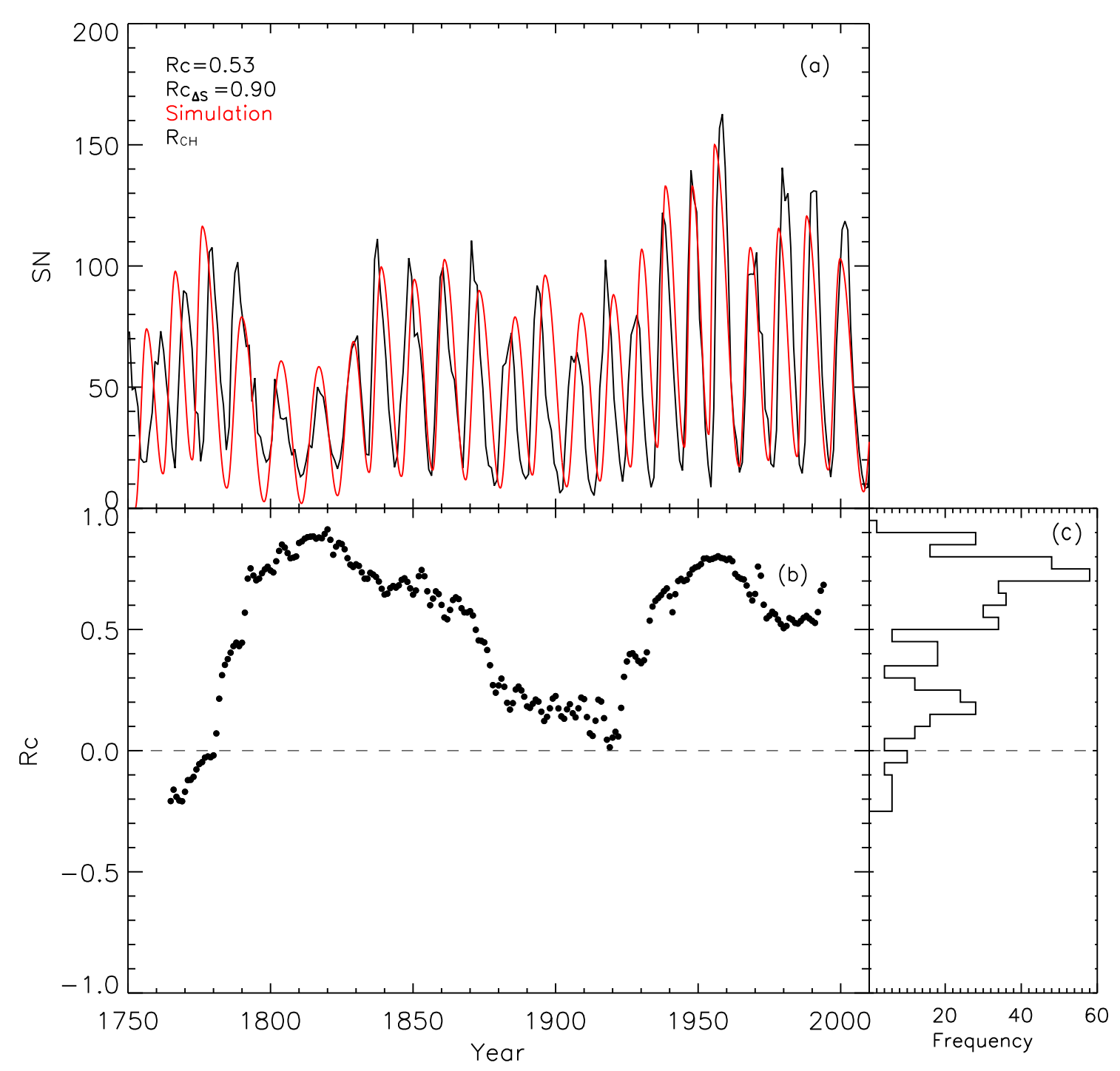

Figure 5.10: Same as Fig. 5.9, but reconstructed based on the final relationships Eqs. (5.6) - (5.9) with $\left\langle R_{\mathrm{CH}}\right\rangle$ series, $\mathrm{H}_{0}$.

series, ${ }^{14} \mathrm{C}$-based U16-14C and the multi-isotope composite Wu18, have the last data point in 1895. The two initial values can therefore be determined from the directly measured SN. In this study, the values of $L_{\mathrm{cyc}, f}$ and $T_{\mathrm{end}, f}$ for these two series are 11.09 and 1902, respectively.

However, the last data point of U16-10Be series (Usoskin et al. 2016a) ends in 1645, which is the beginning of the Maunder minimum and the sunspot was typically zero. Therefore, determining $L_{\mathrm{cyc}, f}$ and $T_{\mathrm{end}, f}$ is not straightforward. Some studies suggested that the Sun may have kept the quasi 11-year cycles during the Maunder minimum although the magnetic activity was not strong enough to form observable sunspots (Beer et al. 1998; Beer 2000a; Fligge et al. 1999; Usoskin et al. 2001; Miyahara et al. 2004; Owens et al. 2012). Some other studies have proposed that the solar dynamo had a different dynamo mode from the present state (Schmitt et al. 1996; Moss et al. 2008; Choudhuri and Karak 2012; Usoskin et al. 2014; Käpylä et al. 2016), while Cameron and Schüssler (2017) recently demonstrated, however, that a different dynamo mode is not required to explain 
the presence and statistics of grand minima. In this study, for the sake of simplicity, we assume that the Sun kept its regular dynamo during the Maunder minimum, i.e., it had a regular cycle length of roughly 11 years $\left(L_{\mathrm{cyc}, f}=11\right)$. In addition, we take 1655 as the solar activity minimum ( $\left.T_{\mathrm{end}, f}\right)$ after the last data point of U16-10Be, by assuming the first observed cycle at the end of the Maunder minimum began around $1699-1700$.

For each isotope input (U16-14C, U16-10Be and Wu18), we simulate the SCs with both $\mathrm{S}_{\mathrm{Nv} 2}$-based and $R_{\mathrm{CH}}$-based relationships, which are taken as upper/lower bounds of the result. The difference between the two simulations is then considered as the uncertainty range. Figure 5.11 shows the results of the simulated SCs based on the three data sets: (a) U16-14C, (b) U16-10Be and (c) Wu18, over the period 1200 - 1900 AD, with their uncertainty shown as shaded areas. The original decadally-averaged SN values of each series are shown by the thick dotted lines. The simulated series in the early epoch, over the period $6800 \mathrm{BC}-6100 \mathrm{BC}$, based on the three data sets are shown in Fig. 5.12.

The uncertainty range values of the simulations based on the three cosmogenic isotope data sets are shown in Fig. 5.13. The annual values for U16-14C, U16-10Be and Wu18 series are shown in light blue, gray and light red, respectively. The 100-year running averages are represented by dark thick curves with the same colours. Additionally, it is seen that the uncertainty values of the Wu18-based simulation are in overall within 10 sunspot numbers, and are smaller than the simulations based on the other two individual isotope series.

These three simulated SN series with quasi 11-year SCs have an annual resolution and are further employed in the semi-empirical model to reconstruct the solar irradiance on millennial time scales.

\subsubsection{Reconstruction of solar irradiance on millennial time scale with solar cycles}

To reconstruct the solar total and spectral irradiance, we use two SATIRE (Spectral And Total Irradiance REconstruction) versions, SATIRE-T (Krivova et al. 2007, 2010) and SATIRE-M (Vieira et al. 2011; Wu et al. 2018a). The SATIRE models have been described in details in many publications, therefore, we only briefly outline the main features of the model here.

SATIRE is a family of semi-empirical models to reconstruct TSI and SSI over wavelength range $115-160000 \mathrm{~nm}$ on different time scales using the most relevant solar activity datasets available at a given time. The model prescribes solar variability on time scales longer than a day to the evolution and the spatial distribution of the solar surface magnetic features. The SATIRE-S version ('S' for Satellite, Krivova et al. 2003; Ball et al. 2012; Yeo et al. 2014b, 2015) uses spatially-resolved full-disc intensity images and magnetograms to determine the sizes and the distributions of the magnetic features on the solar surface and reproduces the measured solar irradiance variability with high accuracy (95\%). Back to the Maunder minimum, the SATIRE-T version (' $\mathrm{T}$ ' for telescope, Krivova et al. 2007, 2010) uses directly observed SN to infer the solar surface magnetisms from a rather simple physical model represented by a set of ordinary differential equations. On time scales of millennia, the SATIRE-M version (' $M$ ' for millennia Vieira and Solanki 2010; Vieira et al. 2011; Wu et al. 2018a) uses decadally-resolved cosmogenic radionuclides in the natural archives as indirect proxies of solar activity. Both, SATIRE-T and 


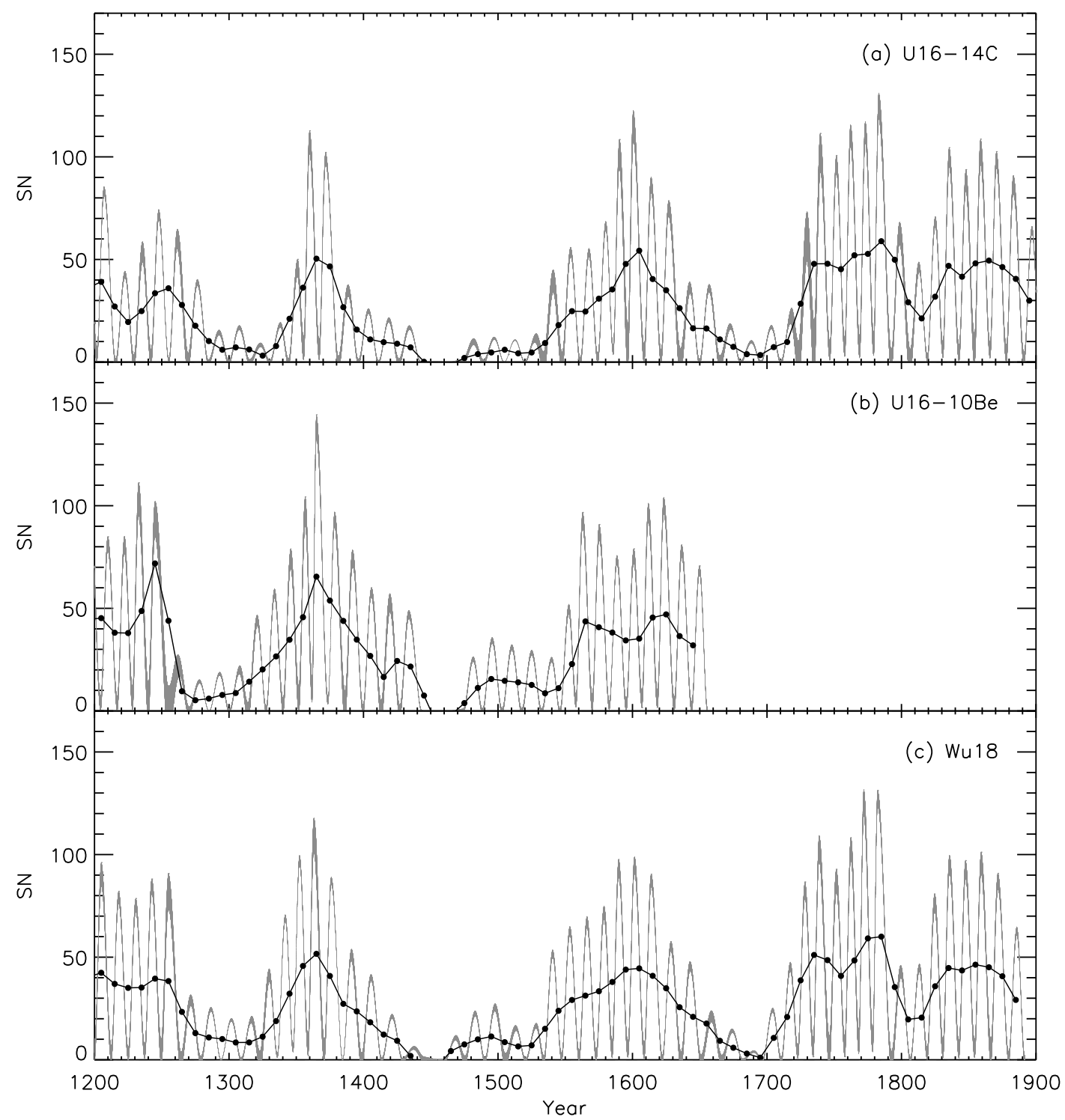

Figure 5.11: Simulated SCs from cosmogenic isotopes between 1200 - 1900 AD. (a) U16-14C. (b) U16-10Be. (c) Wu18. The thick curves with dots are the original decadallyaveraged $\mathrm{SN}$. The simulations with $\mathrm{S}_{\mathrm{Nv} 2}$-based and the $R_{\mathrm{CH}}$-based relationships serve as the upper and lower uncertainties in all three series, shown as shaded area. 


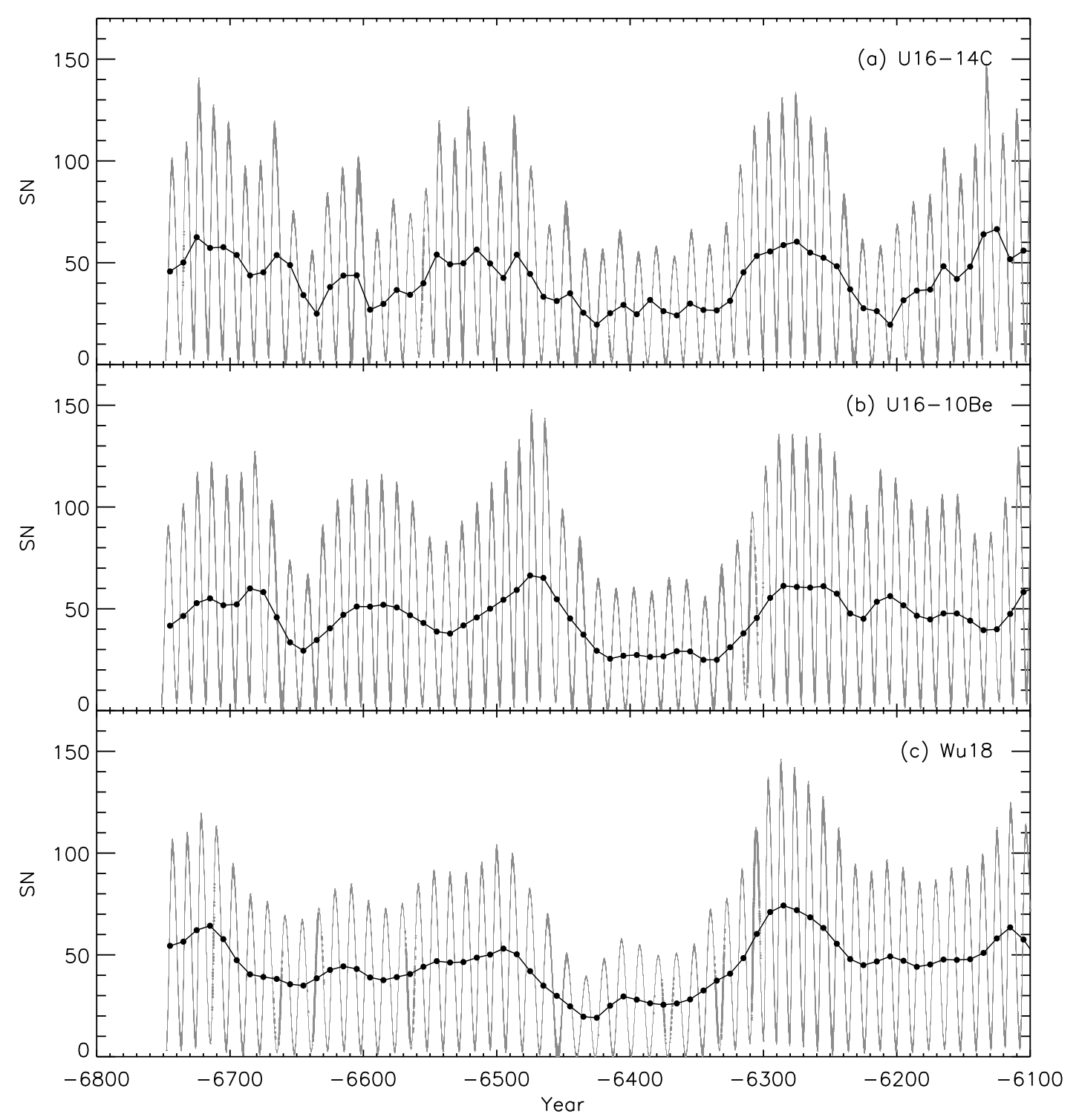

Figure 5.12: Same as Fig. 5.11. Simulated SCs from three cosmogenic isotope-based SN series between $6800-6100$ BC. 


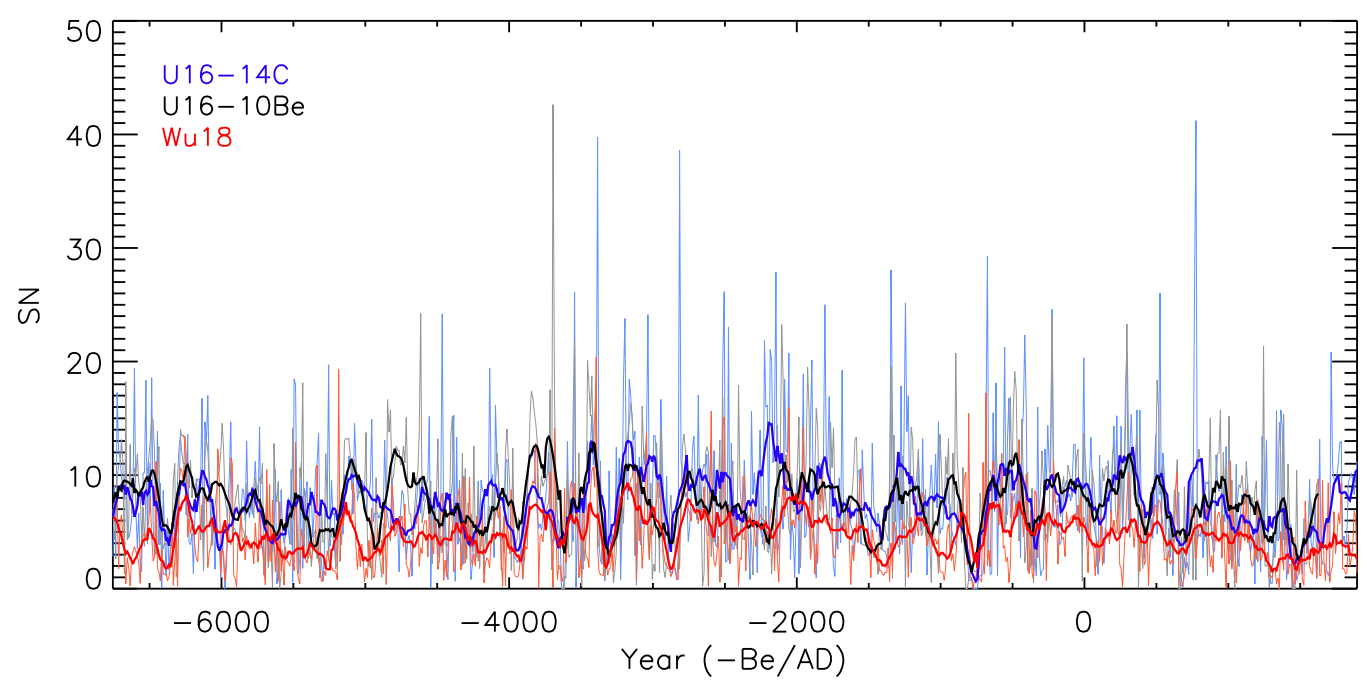

Figure 5.13: Annual values of the uncertainty of the U16-14C (light blue), U16-10Be (gray), and Wu18 dataset-based (light red) over period 6755 BC - 1900 AD. The 100-year running averages of these uncertainties are shown by the darker thick curves of the same colours.

SATIRE-M, employ computations of the evolution of solar total and open magnetic flux according to Solanki et al. (2000, 2002b); Vieira and Solanki (2010).

We refer the reader to paper I for the details on the TSI/SSI reconstruction based on the observed SN with the SATIRE-T model, and the decadal TSI/SSI reconstructions based on the three isotope-based SN with the SATIRE-M model.

The TSI (integral over 115-160000 $\mathrm{nm}$ ) reconstructions based on the three isotope data sets are shown in Fig. 5.14. The panels from top to down are (a) U16-14C, (b) U1610Be and (c) Wu18. The decadal TSI reconstruction with SATIRE-M model are shown as black dotted curves in three panels. Each dot represents a decadal value. Here we improve upon the reconstructions presented in Paper I by starting from annual SN time series reconstructed in Sect. 5.4.1. The presence of the SC and their yearly resolution allows us to employ the SATIRE-T model (Krivova et al. 2007, 2010; Wu et al. 2018a) directly, instead of using the decadal relationship introduced in the SATIRE-M. The TSI reconstructed from these three simulated SCs are also shown in Fig. 5.14: (a) U16-14C, (b) U16-10Be and (c) Wu18. Same as Fig. 5.11, the gray shaded areas represent the difference of the reconstructions with the $\mathrm{S}_{\mathrm{Nv} 2}$-based and the $R_{\mathrm{CH}}$-based relationships. Since the U16-10Be data set ends in 1645, the TSI reconstruction does not overlap with that based on the directly observed SN (Fig. 5.14b). Therefore, the step (3) which is for adjusting the phase, cannot be applied for this reconstruction. For comparison, the 361day running mean TSI reconstruction based on the directly observed $\mathrm{SN},\left\langle\mathrm{S}_{\mathrm{Nv} 2}\right\rangle$, using the SATIRE-T model shown in red in all three panels.

Because the multi-isotope composite $\mathrm{Wu} 18$ accounts for the temporal discrepancies between one global ${ }^{14} \mathrm{C}$ and six regional ${ }^{10} \mathrm{Be}$ series, it averages out many of the systematic effects that individual series suffer from. Hence, it is more robust in representing the solar activity and we recommend the TSI reconstructed from it for the use in paleo-climate 


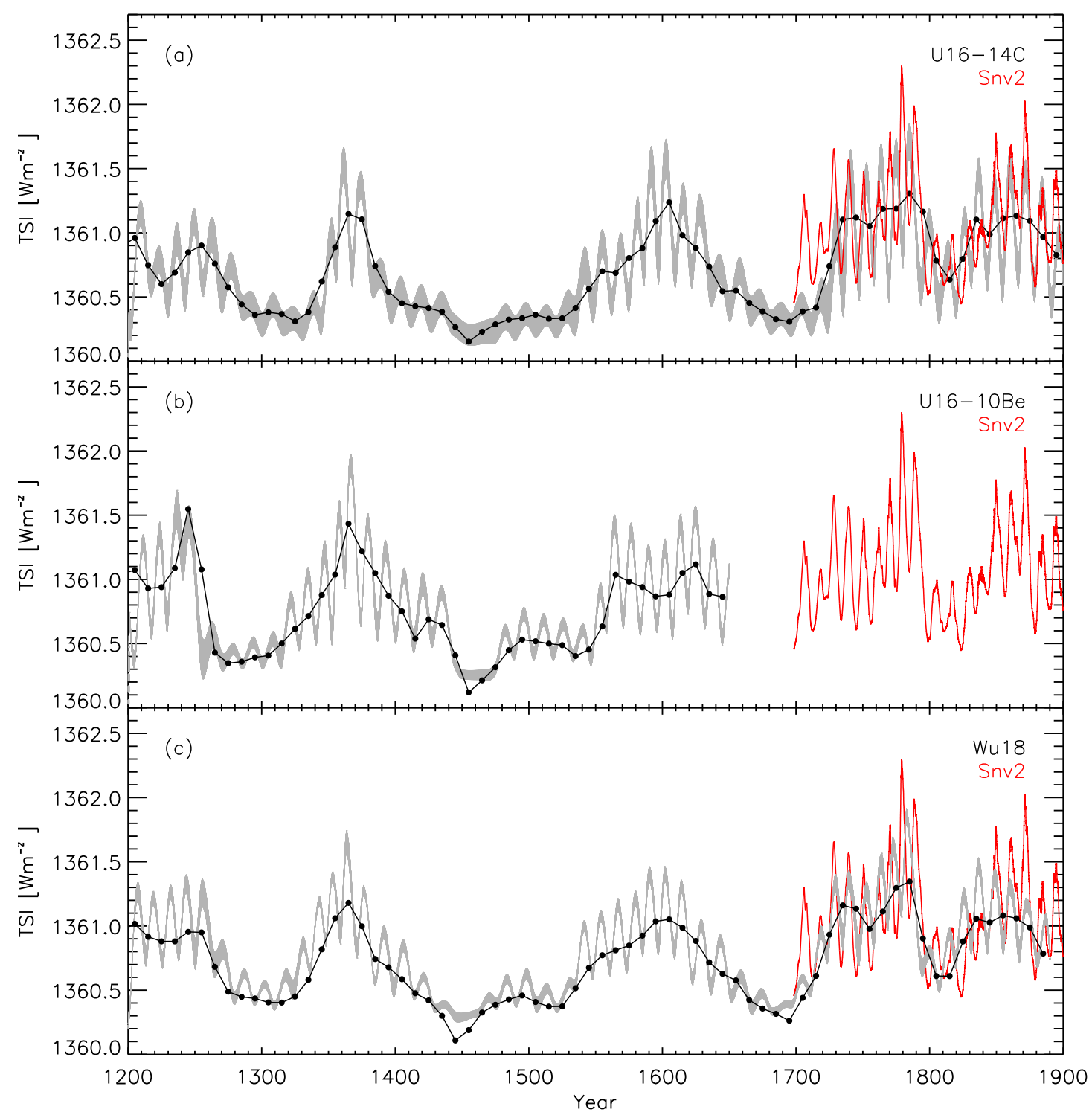

Figure 5.14: TSI reconstructions with SCs from cosmogenic isotopes between 1200 1900 AD using reconstructed SC in Fig. 5.11. (a) U16-14C. (b) U16-10Be. (c) Wu18. The thick curves with dots are the TSI reconstructions with decadally-averaged SN. The $R_{\mathrm{CH}}$-based relationships serve as uncertainty in all three series, indicated by the shaded area. 361-day running average TSI reconstruction based on $S_{\mathrm{Nv} 2}$ is shown in red. 


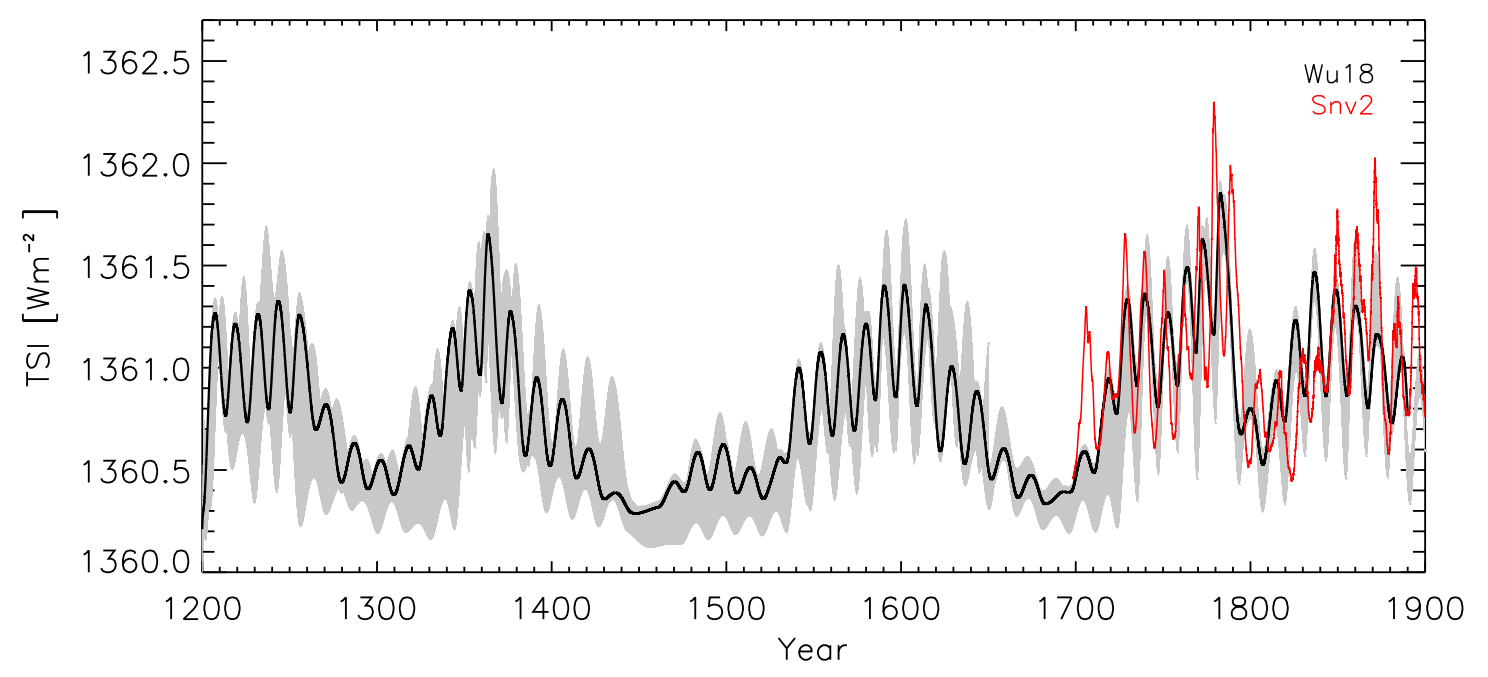

Figure 5.15: TSI reconstruction based on the Wu18 SN series (thick black curve) between $1200-1900$ AD. The upper and lower values among the three reconstructions in Fig. 5.11 at any given time is taken as the uncertainty in this figure (shaded area). 361-day running average TSI reconstruction based on $\mathrm{S}_{\mathrm{Nv} 2}$ is shown in red.

models, as shown by the thick black curve in Fig. 5.15. For the uncertainty, we take the upper and lower values among all three TSI reconstructions (Fig. 5.14) at any given time, shown as shaded area in Fig. 5.15.

The final, recommended TSI and SSI series is obtained by combining the TSI/SSI based on the yearly observed SN since 1700 with the irradiance deduced from yearly isotope-based reconstructed SN over the Holocene.

\subsection{Summary}

The concentration of cosmogenic isotopes, in particular ${ }^{14} \mathrm{C}$ and ${ }^{10} \mathrm{Be}$, in natural archives is a commonly-used indirect proxy of solar activity for times before telescopic measurements of sunspot number started. Various studies have used ${ }^{14} \mathrm{C}$ and ${ }^{10} \mathrm{Be}$, to reconstruct solar activity variation over millennia (e.g., Beer et al. 1988, 2012; Solanki et al. 2004; Steinhilber et al. 2012; Usoskin 2017; Wu et al. 2018b). However, the temporal resolution of those isotope data is usually low, with only decadally-averaged values available. Thus the information on the 11-year solar activity cycle is averaged out. Because the cyclic variation is important for paleo-climate modelling that aims to assess the influence of solar irradiance on the global climate, it is necessary to reconstruct the solar cycles prior to the telescope era.

In this work, we consider four main SC characteristics (the values of solar maxima and solar minima, the rise time and the cycle length) of a directly measured sunspot number series. We used the International sunspot number by (WDC-SILSO, Clette et al. 2014) as a main input record. The new SN series by Chatzistergos et al. (2017) constructed with a non-linear non-parametric method is considered to access the uncertainty range. 
We determine linear relationships between each of these four cycle characteristics and decadally-averaged sunspot number.

Nevertheless, this statistical approach can only gives the timings of the solar activity minima and maxima. In order to obtain a reasonably realistic SC shape, we introduced a first non-parametric fit function, which is inspired by Volobuev (2009). This new nonparametric function describes the general solar cycle shape between both the rising and decaying phase. If the SC characters are known, this function describes the general SC shape with an excellent similarity $\left(R_{\mathrm{c}}=0.96\right)$ to the observed SN over the last 300 years. We then use the four new relationships and the newly derived function describing cycle shape to reconstruct solar cycles from the decadally averaged SN records, initially from artificially averaged directly measured SN.

The TSI reconstructions based on the cosmogenic isotopes with this statistical approach show a good agreement (within uncertainty range) with that based on the observed $\mathrm{SN}$, in both amplitude and the overall trend. However, the errors in the cycle phases accumulate and can lead to an offset of a few years between observed and reconstructed solar cycles.

Finally, using the approach developed and tested here, we reconstruct the yearly SN from the decadally-averaged isotope-based SN over the last 9 millennia. This simulated SN with a yearly cadence is then employed as a proxy of solar activity to reconstruct the TSI/SSI with the SATIRE-T model. This TSI reconstructed from the SN with a simulated SC matches well with the TSI reconstruction based on directly observed SN. 



\title{
6 Summary and outlook
}

\author{
不積跬步, 無以至千里, \\ 不積小流, 無以成江海。 \\ - 简子〈勸學〉
}

In this chapter, we will first summarize the work done in this thesis, which consists of three journal papers. The second part of this chapter will focus on the future plan and the potential projects, which hopefully can improve the results.

\subsection{Summary}

Knowledge of the solar variability on various time scales is critical for better understanding the solar influence on the Earth's climate system. This thesis deals with the solar variability on time scales of centuries and millennia. One of the quantities of interest is the solar irradiance. It has been measured for the last four solar cycles, but this is too short for climate studies. Thus, reconstructions of solar irradiance on longer time scales are needed. This requires long and reliable data and appropriate techniques to reconstruct the irradiance therefrom.

In Chap. 1, we have first introduced the background of the solar structure, the mechanism of solar irradiance, the overview of the measurements and the models to reconstruct solar irradiance. Among all the existing models, SATIRE model is the most successful one. Two of the SATIRE versions, SATIRE-S (Yeo et al. 2014b) and SATIRE-3D (Yeo et al. 2017b), have been amazingly successful in reproducing the observed solar variability, and shown the solar variability on time scales longer than one day is indeed mainly due to the evolution of the surface magnetism. In this thesis, we will use other two SATIRE versions, SATIRE-T (Krivova et al. 2007, 2010) and SATIRE-M (Vieira et al. 2011; Wu et al. 2018a), to reconstruct the solar irradiance on centennial and millennial time scales, respectively. The SATIRE-T model uses the sunspot number as a proxy of solar activity to deduce the emergence rates of the active and ephemeral regions (ARs/ERs). The evolution of solar surface magnetism is then described by a set of ordinary differential equations (ODEs, Solanki et al. 2000, 2002b). The free parameters of the model are constrained by comparing the modelled results to the corresponding reference data sets, using a genetic algorithm PIKAIA. The SATIRE-M model takes the coarser temporal resolution of the input (10 years) into account by averaging the ODEs in the SATIRE-T model decadally. The free parameters obtained from the SATIRE-T model are also used in the SATIRE-M without varying them further. 
The cosmogenic isotopes are produced when the energetic galactic cosmic rays (GCRs) interact with the nuclei in the terrestrial atmosphere. GCRs are modulated by both heliospheric magnetic field and the geomagnetic field. Two isotopes, ${ }^{14} \mathrm{C}$ and ${ }^{10} \mathrm{Be}$ are commonly used for studies of long-term solar activity due to their abundance in natural archives and relatively long half-lives. The two isotopes follow different geochemical paths after their production in the atmosphere. ${ }^{14} \mathrm{C}$ takes part in the global carbon circulation and finally settles in the oceans or the ground, or is absorbed by living organisms. It takes on average around 10 years for ${ }^{14} \mathrm{C}$ to be finally absorbed. ${ }^{10} \mathrm{Be}$ attaches to aerosols and precipitates quickly (usually within $1-2$ years) to the Earth's surface through the wet deposition mechanism. Therefore, the signal in the ${ }^{14} \mathrm{C}$ records is highly globally-mixed while that in ${ }^{10} \mathrm{Be}$ is subject to the local climate at the time when ${ }^{10} \mathrm{Be}$ is settled (Chap. 2).

Owing to the same modulation origin (the Sun and the geomagnetic field), the variation of the signals in both isotope records show a high degree of similarity. Nevertheless, certain short- and mid-term discrepancies can be observed, which are attributed to the systematic effects in the deposition processes. Earlier studies used the individual ${ }^{14} \mathrm{C}$ and ${ }^{10} \mathrm{Be}$ records to estimate the solar long-term variability (Steinhilber et al. 2009, 2012; Vieira et al. 2011; Shapiro et al. 2011; Delaygue and Bard 2011). Some of them have also tried to combine the isotope records. For instance, Delaygue and Bard (2011) averaged two ${ }^{10}$ Be series linearly, and Steinhilber et al. $(2009,2012)$ applied a principal component analysis to extract the common signal among seven records. However, their results are considered less realistic for the following reasons: (1) all the physical processes involved are non-linear while the studies assumed linear isotope-irradiance relationships, (2) the effects from the heliospheric magnetic field and the geomagnetic field cannot be properly disentangled, and (3) the temporal mismatches between individual isotope series were not taken into account by these studies.

To combine the isotope records is neither trivial nor straightforward. In Chap. 3, we have considered one globally-mixed ${ }^{14} \mathrm{C}$ and six local ${ }^{10} \mathrm{Be}$ series sampled in Greenland and Antarctica. Since the concentrations of ${ }^{14} \mathrm{C}$ retrieved from tree rings all over the globe are easier to date and calibrate compared to the individual local ${ }^{10} \mathrm{Be}$ records, we therefore take the ${ }^{14} \mathrm{C}$ series as a reference. We first synchronized all six individual ${ }^{10} \mathrm{Be}$ series with respect to the ${ }^{14} \mathrm{C}$ series by comparing the apparent wiggles (the so-called wiggle-matching method). This is based on the concept that similar wiggles reflect the same modulation origin and therefore they occur around the same time. The correlation coefficient between individual ${ }^{10} \mathrm{Be}$ and the ${ }^{14} \mathrm{C}$ series have improved greatly after the synchronization. The improvement factors for the six series reach up to 2.14.

Next, we employed a new Bayesian approach to calculate the solar activity at any given point of time (described by the solar modulation potential, $\phi$ ). This is done by first using a Monte Carlo simulation to simulate the production rate of the cosmogenic isotopes from any given $\phi$, then searching for the $\phi$ that gives the production rate, which is closest to all the measurements (available individual isotope series at that given point of time). This method allows: (1) to take the geomagnetic field into account, (2) to combine all available series at any given time simultaneously, and (3) to estimate the uncertainty. Further, we have converted the reconstructed solar modulation potential to a more straightforward Sun-related parameter, sunspot number. The reconstructed sunspot number can then be used as the proxy of solar activity on millennial time scales in the 
solar irradiance model, as described in the following.

Using the reconstructed sunspot number from Chap. 3, we used the SATIRE-M model to reconstruct the TSI and SSI on millennial time scales. Since the SATIRE-M model is based partially on the SATIRE-T model, we first re-visited the SATIRE-T model with some significant modifications (Chap. 4). These changes include: the modified physical description of the ER cycle, the more realistic spatial distribution of the ARs, the correction of the UV spectra, and the updates of five used reference data sets.

With the modifications above, the solar irradiance reconstruction with the SATIRE-T model shows an increase of $0.92_{-0.05}^{+0.15} \mathrm{~W} / \mathrm{m}^{2}$ in the TSI between the end of the 17 th century and the present. This value is somewhat lower (but within the uncertainty range) than earlier SATIRE (Krivova et al. 2010; Dasi-Espuig et al. 2016) and NRLSSI (Coddington et al. 2016) return. Compared to the estimates by Shapiro et al. (2010) and Egorova et al. (2018), however, the value is significantly lower, which is due to the different designs in the models.

In addition, the correlation coefficients between the modelled results with the SATIRE$T$ model and the five reference data sets have improved significantly compared with the previous study (Table 4.4). This confirms that the modifications were indeed an improvement.

The parameters fixed in the SATIRE-T model were further employed in the SATIRE$M$ version. We used three isotope records to reconstruct the TSI/SSI over the last nine millennia: (1) official global ${ }^{14} \mathrm{C}$ record (INTCAL13, Roth and Joos 2013), (2) longest ${ }^{10} \mathrm{Be}$ series retrieved from Greenland (GRIP, Yiou et al. 1997; Muscheler et al. 2004; Vonmoos et al. 2006), and (3) the first consistent multi-isotope composite constructed in the Chap.3 (Wu et al. 2018b). We note that the isotope-based reconstructions show better agreement with the reconstruction based on the sunspot number series constructed by Chatzistergos et al. (2017) over the 19th century than that from the latest international sunspot number (Clette et al. 2014). The uniqueness of the reconstruction presented here is that: (1) it is the first-ever isotope-based SSI reconstruction using a physics-based models to describe all the involved physical processes, and (2) it is the first SSI reconstruction based on the first (and so far the only) consistent multi-isotope composite.

The TSI/SSI reconstructions based on cosmogenic isotopes have a lower temporal resolution than reconstructions based on the directly-observed sunspot numbers. As an important input to climate models, the reconstruction with a 10 -year resolution is not ideal. To avoid simulation biases caused by the inconsistency in the temporal resolutions, all energy inputs used in the climate models are required to have at least a 1-year resolution. To account for this issue, Steinhilber et al. (2009) and Schmidt et al. (2011) superimposed a sinusoidal variation and a synthetic solar cycle with a periodicity of 11year, respectively, on the decadally-averaged sunspot number. However, these methods are not very realistic.

In the last part of this thesis (Chap. 5), we developed a statistical approach to simulate the realistic quasi 11-year solar cycle in the past, for climate applications. First, we characterised the solar cycle with four properties from the annual international sunspot number: (1) sunspot maximum, (2) sunspot minimum, (3) cycle length, and (4) cycle rising time. The four properties showed good correlations with the corresponding decadally-averaged sunspot numbers (Fig. 5.2). As a result, a set of four relationships were obtained, allowing us to simulate the solar cycles back in time based solely on the 
decadally-averaged sunspot number values. We have also tested the temporal sensitivity of this statistical approach. In addition, inspired by the one-parameter function by Volobuev (2009), we introduced the first solar cycle shape function that does not require any free parameters. This non-parametric function shows an excellent agreement with the directly-observed sunspot number cycle shape $\left(R_{\mathrm{c}}=0.96\right)$, which we use to validate the function.

This method was then applied to simulate the solar cycle based on the decadal sunspot number reconstructed from the cosmogenic isotopes. The simulated series with quasi 11-year variation has 1-year resolution. They were fed in the SATIRE-T model to reconstruct the annual solar irradiance. The TSI/SSI reconstructions from the isotope-based sunspot number with solar cycles showed excellent agreement with that from the directlyobserved sunspot number, in both long-term trend and amplitude (Fig. 5.15). However, the simulated series show some discrepancies in the phases compared to the direct observations. This is, unfortunately, inevitable because the cycle length is determined from a rather simplistic linear relationship, which results in the errors propagating back in time.

To sum up, this thesis has provided (1) the first multi-isotope composite and the sunspot number computed from it, (2) the first full SSI reconstruction on the millennial time scale using a series of physics-based models, and (3) the first realistically-simulated quasi 11-year solar cycle variation on the millennial time scale using a statistical method. The reconstructed TSI and SSI with annual resolution have been provided as solar radiative forcing input in the Paleoclimate Modelling Intercomparison Project-4 (PMIP4), which will help to better understand the influence of the solar variability on Earth's climate.

All the TSI/SSI reconstructions obtained in this thesis are (to be) published and will be available on the "Solar Variability and Climate" webpage ${ }^{1}$

\subsection{Outlook}

When computing the solar modulation potential for the individual cosmogenic isotope data sets, we noticed opposite behaviours in the two longest ${ }^{10} \mathrm{Be}$ series: the GRIP and the EDML. Compared to the geomagnetic field (VADM) over the last 9000 years, the EDML series shows the similar long-term trend as seen in the VADM series, whereas the GRIP series shows an opposite trend (Fig. 3.8). This discrepancy might imply either an under-correction of the geomagnetic shielding for Greenland region, or an unsuitable atmospheric transport/deposition model for the Greenland drilling site. This issue might be solved by applying a full-sized atmospheric transport/deposition model (Sukhodolov et al. 2017). Furthermore, the new Bayesian statistical model employed in this thesis has the advantage of combining multiple available cosmogenic isotope series at any given time. Hence, we hope to make use of this statistical method to combine more cosmogenic isotope data sets in the future (e.g., Beer et al. 1983, 1988; Finkel and Nishiizumi 1997).

As discussed in Chap. 4, the secular change in the solar irradiance from the end of the 17 th century to the modern maximum returned by various models shows a wide range and large uncertainties. Most models that use sunspot numbers as input have roughly similar estimates, but then reach down to a saturation (quiet-Sun) level during the Maunder minimum since the sunspot number drops to, and stays over longer periods of time at zero.

\footnotetext{
${ }^{1}$ http: //www2 .mps.mpg.de/projects/sun-climate/data.html
} 
However, this is probably not very realistic and is rather an artefact of the model design and the lack of information on the sunspot number.

There are various cosmogenic isotope data sets showing signals during the Maunder minimum. This implies that the small-scale magnetic features could still be present even though the solar magnetic activity was too weak to form many observable sunspots. However, it is very difficult to estimate how many of those small-scaled magnetic features existed on the solar surface during grand minima. The yearly active day fraction (ADF), which is defined as the ratio of days with observed sunspots to the number of observation days (e.g., Vaquero et al. 2015; Usoskin et al. 2016c), can in principle carry some information on the solar activity. Compared to the directly observed sunspot number record which has too many non-spot records and gaps during the Maunder minimum, the yearly and triennial ADF values are usually not zero (see Fig.1 in Vaquero et al. 2015). This supports that the existence of the weak solar activity, which can probably be recovered. For instance, we can employ the ADF values to describe the emergence rates of ARs/ERs instead of using the daily sunspot numbers (Eqs. 4.6 and 4.7), for the Maunder minimum period. Additionally, some isotope data records that cover the Maunder minimum period have higher resolutions of about 1 - 2 years (Beer et al. 1990; Beer 2000b). We could, in theory, also use such signals in the isotope data to represent the weak solar activity. However, one should note that the signals in the isotope data sets with higher temporal resolutions are also highly influenced by the local climate conditions, which should be properly accounted for before any statistical analysis.

Finally, although the amplitude and the overall trend of solar activity can be simulated reasonably well with the statistical approach described in Chap. 5, this method could still be refined. In Chap. 5, we have pointed out that the solar cycle phase cannot be always recovered, particularly when a grand minimum is encountered. This is because we used linear regression to describe the relationship between the decadally-averaged sunspot number and the cycle length. As a consequence, the errors accumulate easily back in time. This shortcoming could possibly be tackled in the following ways. First, using the cosmogenic isotope data with higher temporal resolutions to determine the possible solar cycles during the Maunder minimum, we can refine our existing statistical approach (Sect. 5.3.1) and constrain the relationship between the decadally-averaged sunspot number and the cycle length. Second, Solanki et al. (2002a) indicated that the relationship between the cycle length and cycle amplitude is much more complex than a linear relationship. They have constructed a simple empirical relationship, showing the amplitude of a given cycle can be described relatively well by the lengths of earlier cycles. Such an empirical relationship might require knowledge of lengths of the latest two cycle as initial inputs, but it would not be a problem for the multi-isotope composite (Wu et al. 2018b) since this series ends in 1895. Therefore, we can introduce this empirical relationship into our statistical approach, and improve the steps of simulating the lengths of the previous cycles. 



\section{Bibliography}

Abbot, C. G.: 1923, Mon. Wea. Rev. 51, 71

Adolphi, F. and Muscheler, R.: 2016, Clim. Past 12(1), 15

Adriani, O., Barbarino, G. C., Bazilevskaya, G. A., Bellotti, R., Bianco, A., Boezio, M., Bogomolov, E. A., Bongi, M., Bonvicini, V., Bottai, S., Bruno, A., Cafagna, F., Campana, D., Carbone, R., Carlson, P., Casolino, M., Castellini, G., De Donato, C., De Santis, C., De Simone, N., Di Felice, V., Formato, V., Galper, A. M., Karelin, A. V., Koldashov, S. V., Koldobskiy, S. A., Krutkov, S. Y., Kvashnin, A. N., Leonov, A., Malakhov, V., Marcelli, L., Martucci, M., Mayorov, A. G., Menn, W., Mergé, M., Mikhailov, V. V., Mocchiutti, E., Monaco, A., Mori, N., Munini, R., Osteria, G., Palma, F., Papini, P., Pearce, M., Picozza, P., Pizzolotto, C., Ricci, M., Ricciarini, S. B., Rossetto, L., Sarkar, R., Scotti, V., Simon, M., Sparvoli, R., Spillantini, P., Stochaj, S. J., Stockton, J. C., Stozhkov, Y. I., Vacchi, A., Vannuccini, E., Vasilyev, G. I., Voronov, S. A., Yurkin, Y. T., Zampa, G., Zampa, N., and Zverev, V. G.: 2013, Phys. Rev. Lett. 111(8), 081102

Agostinelli, S., Allison, J., Amako, K., Apostolakis, J., Araujo, H., Arce, P., Asai, M., Axen, D., Banerjee, S., Barrand, G., Behner, F., Bellagamba, L., Boudreau, J., Broglia, L., Brunengo, A., Burkhardt, H., Chauvie, S., Chuma, J., Chytracek, R., Cooperman, G., Cosmo, G., Degtyarenko, P., Dell'Acqua, A., Depaola, G., Dietrich, D., Enami, R., Feliciello, A., Ferguson, C., Fesefeldt, H., Folger, G., Foppiano, F., Forti, A., Garelli, S., Giani, S., Giannitrapani, R., Gibin, D., Gómez Cadenas, J. J., González, I., Gracia Abril, G., Greeniaus, G., Greiner, W., Grichine, V., Grossheim, A., Guatelli, S., Gumplinger, P., Hamatsu, R., Hashimoto, K., Hasui, H., Heikkinen, A., Howard, A., Ivanchenko, V., Johnson, A., Jones, F. W., Kallenbach, J., Kanaya, N., Kawabata, M., Kawabata, Y., Kawaguti, M., Kelner, S., Kent, P., Kimura, A., Kodama, T., Kokoulin, R., Kossov, M., Kurashige, H., Lamanna, E., Lampén, T., Lara, V., Lefebure, V., Lei, F., Liendl, M., Lockman, W., Longo, F., Magni, S., Maire, M., Medernach, E., Minamimoto, K., Mora de Freitas, P., Morita, Y., Murakami, K., Nagamatu, M., Nartallo, R., Nieminen, P., Nishimura, T., Ohtsubo, K., Okamura, M., O’Neale, S., Oohata, Y., Paech, K., Perl, J., Pfeiffer, A., Pia, M. G., Ranjard, F., Rybin, A., Sadilov, S., Di Salvo, E., Santin, G., Sasaki, T., Savvas, N., Sawada, Y., Scherer, S., Sei, S., Sirotenko, V., Smith, D., Starkov, N., Stoecker, H., Sulkimo, J., Takahata, M., Tanaka, S., Tcherniaev, E., Safai Tehrani, E., Tropeano, M., Truscott, P., Uno, H., Urban, L., Urban, P., Verderi, M., Walkden, A., Wander, W., Weber, H., Wellisch, J. P., Wenaus, T., Williams, D. C., Wright, D., Yamada, T., Yoshida, H., Zschiesche, D., and G EANT4 Collaboration: 2003, Nuclear Instruments and Methods in Physics Research A 506, 250 
Aguilar, M., Aisa, D., Alpat, B., Alvino, A., Ambrosi, G., Andeen, K., Arruda, L., Attig, N., Azzarello, P., Bachlechner, A., and et al.: 2015, Phys. Rev. Lett. 114(17), 171103

Alcaraz, J., Alpat, B., Ambrosi, G., Anderhub, H., Ao, L., Arefiev, A., Azzarello, P., Babucci, E., Baldini, L., Basile, M., Barancourt, D., Barao, F., Barbier, G., Barreira, G., Battiston, R., Becker, R., Becker, U., Bellagamba, L., Béné, P., Berdugo, J., Berges, P., Bertucci, B., Biland, A., Bizzaglia, S., Blasko, S., Boella, G., Boschini, M., Bourquin, M., Brocco, L., Bruni, G., Buenerd, M., Burger, J. D., Burger, W. J., Cai, X. D., Camps, C., Cannarsa, P., Capell, M., Casadei, D., Casaus, J., Castellini, G., Cecchi, C., Chang, Y. H., Chen, H. F., Chen, H. S., Chen, Z. G., Chernoplekov, N. A., Chiueh, T. H., Chuang, Y. L., Cindolo, F., Commichau, V., Contin, A., Crespo, P., Cristinziani, M., da Cunha, J. P., Dai, T. S., Deus, J. D., Dinu, N., Djambazov, L., D’ Antone, I., Dong, Z. R., Emonet, P., Engelberg, J., Eppling, F. J., Eronen, T., Esposito, G., Extermann, P., Favier, J., Fiandrini, E., Fisher, P. H., Fluegge, G., Fouque, N., Galaktionov, Y., Gervasi, M., Giusti, P., Grandi, D., Grimm, O., Gu, W. Q., Hangarter, K., Hasan, A., Hermel, V., Hofer, H., Huang, M. A., Hungerford, W., Ionica, M., Ionica, R., Jongmanns, M., Karlamaa, K., Karpinski, W., Kenney, G., Kenny, J., Kim, W., Klimentov, A., Kossakowski, R., Koutsenko, V., Kraeber, M., Laborie, G., Laitinen, T., Lamanna, G., Laurenti, G., Lebedev, A., Lee, S. C., Levi, G., Levtchenko, P., Liu, C. L., Liu, H. T., Lopes, I., Lu, G., Lu, Y. S., Lübelsmeyer, K., Luckey, D., Lustermann, W., Maña, C., Margotti, A., Mayet, F., McNeil, R. R., Meillon, B., Menichelli, M., Mihul, A., Mourao, A., Mujunen, A., Palmonari, F., Papi, A., Park, I. H., Pauluzzi, M., Pauss, F., Perrin, E., Pesci, A., Pevsner, A., Pimenta, M., Plyaskin, V., Pojidaev, V., Pohl, M., Postolache, V., Produit, N., Rancoita, P. G., Rapin, D., Raupach, F., Ren, D., Ren, Z., Ribordy, M., Richeux, J. P., Riihonen, E., Ritakari, J., Roeser, U., Roissin, C., Sagdeev, R., Sartorelli, G., Schultz von Dratzig, A., Schwering, G., Scolieri, G., Seo, E. S., Shoutko, V., Shoumilov, E., Siedling, R., Son, D., Song, T., Steuer, M., Sun, G. S., Suter, H., Tang, X. W., Ting, S. C. C., Ting, S. M., Tornikoski, M., Torsti, J., Trümper, J., Ulbricht, J., Urpo, S., Usoskin, I., Valtonen, E., Vandenhirtz, J., Velcea, F., Velikhov, E., Verlaat, B., Vetlitsky, I., Vezzu, F., Vialle, J. P., Viertel, G., Vité, D., Von Gunten, H., Waldmeier Wicki, S., Wallraff, W., Wang, B. C., Wang, J. Z., Wang, Y. H., Wiik, K., Williams, C., Wu, S. X., Xia, P. C., Yan, J. L., Yan, L. G., Yang, C. G., Yang, M., Ye, S. W., Yeh, P., Xu, Z. Z., Zhang, H. Y., Zhang, Z. P., Zhao, D. X., Zhu, G. Y., Zhu, W. Z., Zhuang, H. L., Zichichi, A., and Zimmermann, B.: 2000, Physics Letters $B$ 490, 27

Alfvén, H.: 1947, MNRAS 107, 211

Allison, J., Amako, K., Apostolakis, J., Araujo, H., Dubois, P. A., Asai, M., Barrand, G., Capra, R., Chauvie, S., Chytracek, R., Cirrone, G. A. P., Cooperman, G., Cosmo, G., Cuttone, G., Daquino, G. G., Donszelmann, M., Dressel, M., Folger, G., Foppiano, F., Generowicz, J., Grichine, V., Guatelli, S., Gumplinger, P., Heikkinen, A., Hrivnacova, I., Howard, A., Incerti, S., Ivanchenko, V., Johnson, T., Jones, F., Koi, T., Kokoulin, R., Kossov, M., Kurashige, H., Lara, V., Larsson, S., Lei, F., Link, O., Longo, F., Maire, M., Mantero, A., Mascialino, B., McLaren, I., Lorenzo, P. M., Minamimoto, K., Murakami, K., Nieminen, P., Pandola, L., Parlati, S., Peralta, L., Perl, J., Pfeiffer, A., Pia, M. G., Ribon, A., Rodrigues, P., Russo, G., Sadilov, S., Santin, G., Sasaki, T., 
Smith, D., Starkov, N., Tanaka, S., Tcherniaev, E., Tome, B., Trindade, A., Truscott, P., Urban, L., Verderi, M., Walkden, A., Wellisch, J. P., Williams, D. C., Wright, D., and Yoshida, H.: 2006, IEEE Transactions on Nuclear Science 53, 270

Allison, J., Amako, K., Apostolakis, J., Arce, P., Asai, M., Aso, T., Bagli, E., Bagulya, A., Banerjee, S., Barrand, G., Beck, B. R., Bogdanov, A. G., Brandt, D., Brown, J. M. C., Burkhardt, H., Canal, P., Cano-Ott, D., Chauvie, S., Cho, K., Cirrone, G. A. P., Cooperman, G., Cortés-Giraldo, M. A., Cosmo, G., Cuttone, G., Depaola, G., Desorgher, L., Dong, X., Dotti, A., Elvira, V. D., Folger, G., Francis, Z., Galoyan, A., Garnier, L., Gayer, M., Genser, K. L., Grichine, V. M., Guatelli, S., Guèye, P., Gumplinger, P., Howard, A. S., Hřivnáčová, I., Hwang, S., Incerti, S., Ivanchenko, A., Ivanchenko, V. N., Jones, F. W., Jun, S. Y., Kaitaniemi, P., Karakatsanis, N., Karamitrosi, M., Kelsey, M., Kimura, A., Koi, T., Kurashige, H., Lechner, A., Lee, S. B., Longo, F., Maire, M., Mancusi, D., Mantero, A., Mendoza, E., Morgan, B., Murakami, K., Nikitina, T., Pandola, L., Paprocki, P., Perl, J., Petrović, I., Pia, M. G., Pokorski, W., Quesada, J. M., Raine, M., Reis, M. A., Ribon, A., Ristić Fira, A., Romano, F., Russo, G., Santin, G., Sasaki, T., Sawkey, D., Shin, J. I., Strakovsky, I. I., Taborda, A., Tanaka, S., Tomé, B., Toshito, T., Tran, H. N., Truscott, P. R., Urban, L., Uzhinsky, V., Verbeke, J. M., Verderi, M., Wendt, B. L., Wenzel, H., Wright, D. H., Wright, D. M., Yamashita, T., Yarba, J., and Yoshida, H.: 2016, Nuclear Instruments and Methods in Physics Research A 835, 186

Anderson, E. C., Libby, W. F., Weinhouse, S., Reid, A. F., Kirshenbaum, A. D., and Grosse, A. V.: 1947, Science 105, 576

Arge, C. N., Hildner, E., Pizzo, V. J., and Harvey, J. W.: 2002, J. Geophys. Res. 107, 1319

Arnold, J. R.: 1956, Science 124, 584

Asvestari, E., Gil, A., Kovaltsov, G. A., and Usoskin, I. G.: 2017, J. Geophys. Res. 122, 9790

Avrett, E. H. and Loeser, R.: 1992, in M. S. Giampapa and J. A. Bookbinder (eds.), Cool Stars, Stellar Systems, and the Sun, Vol. 26 of Astronomical Society of the Pacific Conference Series, p. 489

Axford, W. I., Petschek, H. E., and Siscoe, G. L.: 1965, J. Geophys. Res. 70, 1231

Baade, W. and Zwicky, F.: 1934, Proceedings of the National Academy of Science 20, 254

Babcock, H. W.: 1948, Phys. Rev. 74, 489

Babcock, H. W.: 1961, ApJ 133, 572

Babu, G. J. and Feigelson, E. D.: 1992, Communications in Statistics - Simulation and Computation 21(2), 533

Backus, G., Parker, R., and Constable, C.: 1996, Foundations of Geomagnetism, Cambridge University Press 
Ball, W. T., Krivova, N. A., Unruh, Y. C., Haigh, J. D., and Solanki, S. K.: 2014, J. Atmos. Sci. 71, 4086

Ball, W. T., Unruh, Y. C., Krivova, N. A., Solanki, S. K., Wenzler, T., Mortlock, D. J., and Jaffe, A. H.: 2012, AEFA 541, A27

Balmaceda, L., Krivova, N. A., and Solanki, S. K.: 2007, Adv. Space Res. 40, 986

Balmaceda, L., Solanki, S. K., and Krivova, N. A.: 2005, Memorie della Societa Astronomica Italiana 76, 929

Balmaceda, L. A., Solanki, S. K., Krivova, N. A., and Foster, S.: 2009, J. Geophys. Res. 114, A07104

Bard, E., Raisbeck, G. M., Yiou, F., and Jouzel, J.: 1997, Earth Planet. Sci. Lett. 150, 453

Battistoni, G., Boehlen, T., Cerutti, F., Chin, P. W., Salvatore Esposito, L., Fassò, A., Ferrari, A., Mereghetti, A., Garcia Ortega, P., Ranft, J., Roesler, S., Sala, P. R., and Vlachoudis, V.: 2014, in Joint International Conference on Supercomputing in Nuclear Applications + Monte Carlo, p. 06005, EDP Sciences

Baumann, I., Schmitt, D., Schüssler, M., and Solanki, S. K.: 2004, AEFA 426(3), 1075

Bazilevskaya, G. A., Cliver, E. W., Kovaltsov, G. A., Ling, A. G., Shea, M. A., Smart, D. F., and Usoskin, I. G.: 2014, Space Sci. Rev. 186, 409

Bazin, L., Landais, A., Lemieux-Dudon, B., Toyé Mahamadou Kele, H., Veres, D., Parrenin, F., Martinerie, P., Ritz, C., Capron, E., Lipenkov, V., Loutre, M.-F., Raynaud, D., Vinther, B., Svensson, A., Rasmussen, S. O., Severi, M., Blunier, T., Leuenberger, M., Fischer, H., Masson-Delmotte, V., Chappellaz, J., and Wolff, E.: 2013, Clim. Past 9, 1715

Beer, J.: 2000a, Space Sci. Rev. 94, 53

Beer, J.: 2000b, in The Solar Cycle and Terrestrial Climate, Solar and Space weather, Vol. 463 of ESA Special Publication, p. 671

Beer, J., Blinov, A., Bonani, G., Hofmann, H. J., and Finkel, R. C.: 1990, Nature 347, 164

Beer, J., McCracken, K., and von Steiger, R.: 2012, Cosmogenic Radionuclides, Springer, Berlin, Heidelberg

Beer, J., Siegenthaler, U., Oeschger, H., Andree, M., Bonani, G., Suter, M., Wolfli, W., Finkel, R. C., and Langway, C. C.: 1983, International Cosmic Ray Conference 9, 317

Beer, J., Siegenthaler, U., Oeschger, H., Bonani, G., and Finkel, R. C.: 1988, Nature 331, 675

Beer, J., Tobias, S., and Weiss, N.: 1998, Sol. Phys. 181, 237 
Beer, J., Vonmoos, M., and Muscheler, R.: 2006, Space Sci. Rev. 125, 67

Berggren, A. M., Beer, J., Possnert, G., Aldahan, A., Kubik, P., Christl, M., Johnsen, S. J., Abreu, J., and Vinther, B. M.: 2009, Geophys. Res. Lett. 36, L11801

Bertolotti, M.: 2013, The Discovery: Victor F. Hess and the Balloon Ascents, pp 33-44, Springer Berlin Heidelberg, Berlin, Heidelberg

Bhandari, N., Lal, D., and Rama: 1966, Tellus 18, 391

Biermann, L.: 1948, ZAp 25, 161

Biermann, P. L. and Strittmatter, P. A.: 1987, ApJ 322, 643

Biswas, S.: 2000, Cosmic Perspectives in Space Physics, Astrophysics and Space Science Library, Springer

Bolin, B., Degens, E. T., Kempe, S., and Ketner, P.: 1979, Global carbon cycle: SCOPE 13, John Wiley and Sons, New York, NY

Bond, G., Kromer, B., Beer, J., Muscheler, R., Evans, M. N., Showers, W., Hoffmann, S., Lotti-Bond, R., Hajdas, I., and Bonani, G.: 2001, Science 294, 2130

Brueckner, G. E., Edlow, K. L., Floyd, IV, L. E., Lean, J. L., and Vanhoosier, M. E.: 1993, J. Geophys. Res. 98, 10

Buffett, B. A.: 2000, Science 288, 2007

Burger, R. A., Potgieter, M. S., and Heber, B.: 2000, J. Geophys. Res. 105, 27447

Caballero-Lopez, R. A. and Moraal, H.: 2004, J. Geophys. Res. 109, A01101

Cain, W. F. and Suess, H. E.: 1976, J. Geophys. Res. 81, 3688

Cameron, R. H., Dasi-Espuig, M., Jiang, J., Iş1k, E., Schmitt, D., and Schüssler, M.: 2013, $A \mathcal{E} A$ 557, A141

Cameron, R. H., Jiang, J., Schmitt, D., and Schüssler, M.: 2010, ApJ 719, 264

Cameron, R. H. and Schüssler, M.: 2017, ApJ 843, 111

Carlsson, M., Stein, R. F., Nordlund, A. A., and Scharmer, G. B.: 2004, ApJ 610, L137

Carmichael, H., Bercovitch, M., Shea, M. A., Magidin, M., and Peterson, R. W.: 1968, Can. J. Phys. 46, 1006

Cebula, R. P., Deland, M. T., and Schlesinger, B. M.: 1992, J. Geophys. Res. 97, 11

Chapman, G. A. and Boyden, J. E.: 1986, ApJ 302, L71

Chapman, G. A., Cookson, A. M., and Dobias, J. J.: 1996, J. Geophys. Res. 101, 13541

Chapman, G. A., Cookson, A. M., and Dobias, J. J.: 1997, ApJ 482, 541 
Chapman, G. A., Cookson, A. M., and Preminger, D. G.: 2012, Sol. Phys. 276, 35

Chapman, G. A., Cookson, A. M., and Preminger, D. G.: 2013, Sol. Phys. 283, 295

Chapman, S. and Bartels, J.: 1940, Geomagnetism, Vol. I: Geomagnetic and Related Phenomena, Oxford Univ. Press

Charbonneau, P.: 1995, ApJS 101, 309

Charbonneau, P.: 2010, Liv. Rev. Sol. Phys. 7, 3

Charbonnel, C., Däppen, W., Schaerer, D., Bernasconi, P. A., Maeder, A., Meynet, G., and Mowlavi, N.: 1999, AEFAS 135, 405

Chatzistergos, T., Ermolli, I., Solanki, S. K., and Krivova, N. A.: 2018, AE्EA 609, A92

Chatzistergos, T., Usoskin, I. G., Kovaltsov, G. A., Krivova, N. A., and Solanki, S. K.: 2017, AE्FA 602, A69

Choudhuri, A. R. and Karak, B. B.: 2012, Phys. Rev. Lett. 109, 171103

Christensen-Dalsgaard, J.: 2002, Rev. Mod. Phys. 74, 1073

Clement, B. M.: 2004, Nature 428, 637

Clette, F. and Lefévre, L.: 2016, Sol. Phys. 291, 2629

Clette, F., Svalgaard, L., Vaquero, J. M., and Cliver, E. W.: 2014, Space Sci. Rev. 186, 35

Cliver, E. W. and Ling, A. G.: 2016, Sol. Phys. 291, 2763

Coddington, O., Lean, J. L., Pilewskie, P., Snow, M., and Lindholm, D.: 2016, Bull. Amer. Meteor. Soc. 97, 1265

Cohen, J.: 2003, Applied Multiple Regression/correlation Analysis for the Behavioral Sciences, No. v. 1 in Applied Multiple Regression/correlation Analysis for the Behavioral Sciences, Routledge

Compton, A. H., Wollan, E. O., and Bennett, R. D.: 1934, Review of Scientific Instruments 5,415

Constable, C.: 2007, Dipole moment variation, pp 159-161, Encyclopedia of earth sciences series, Springer

Cooke, D. J.: 1974, Proceedings of the Astronomical Society of Australia 2, 296

Cooke, D. J., Humble, J. E., Shea, M. A., Smart, D. F., and Lund, N.: 1991, Nuovo Cimento C Geophysics Space Physics C 14, 213

Crucifix, M., Loutre, M. F., and Berger, A.: 2006, Space Sci. Rev. 125, 213

Cubasch, U., Voss, R., Hegerl, G. C., Waszkewitz, J., and Crowley, T. J.: 1997, Climate Dynamics 13(11), 757 
Dahl-Jensen, D., Gundestrup, N. S., Miller, H., Watanabe, O., Johnsen, S. J., Steffensen, J. P., Clausen, H. B., Svensson, A., and Larsen, L. B.: 2002, Annals of Glaciology 35, 1

Damon, P. E. and Jirikowic, J. L.: 1992, Radiocarbon 34(2), 199

Damon, P. E., Lerman, J. C., and Long, A.: 1978, Ann. Rev. Earth Planet. Sci. Lett. 6, 457

Damon, P. E. and Sonett, C. P.: 1991, in C. P. Sonett, M. S. Giampapa, and M. S. Matthews (eds.), The Sun in Time, pp 360-388

Dasi-Espuig, M., Jiang, J., Krivova, N. A., and Solanki, S. K.: 2014, AEFA 570, A23

Dasi-Espuig, M., Jiang, J., Krivova, N. A., Solanki, S. K., Unruh, Y. C., and Yeo, K. L.: 2016, AEFA 590, A63

Delaygue, G. and Bard, E.: 2011, Clim. Dynam. 36, 2201

Dessler, A. J. and Juday, R. D.: 1965, Planet. Space Sci. 13, 63

Dewitte, S., Crommelynck, D., Mekaoui, S., and Joukoff, A.: 2004, Sol. Phys. 224, 209

Dicke, R. H.: 1978, Nature 276, 676

Dickinson, R. E.: 1975, Bull. Am. Meteorol. Soc 56, 1240

Dikpati, M. and Gilman, P. A.: 2007, Sol. Phys. 241, 1

Domingo, V., Ermolli, I., Fox, P., Fröhlich, C., Haberreiter, M., Krivova, N. A., Kopp, G., Schmutz, W., Solanki, S. K., Spruit, H. C., Unruh, Y. C., and Vögler, A.: 2009, Space Sci. Rev. 145, 337

Dorman, I. V. and Dorman, L. I.: 1967, J. Geophys. Res. 72, 1513

Dorman, L. I. (ed.): 2004, Cosmic Rays in the Earth's Atmosphere and Underground, Vol. 303 of Astrophysics and Space Science Library

Dorman, L. I.: 2012, Annales Geophysicae 30, 9

Dunne, E. M., Gordon, H., Kürten, A., Almeida, J., Duplissy, J., Williamson, C., Ortega, I. K., Pringle, K. J., Adamov, A., Baltensperger, U., Barmet, P., Benduhn, F., Bianchi, F., Breitenlechner, M., Clarke, A., Curtius, J., Dommen, J., Donahue, N. M., Ehrhart, S., Flagan, R. C., Franchin, A., Guida, R., Hakala, J., Hansel, A., Heinritzi, M., Jokinen, T., Kangasluoma, J., Kirkby, J., Kulmala, M., Kupc, A., Lawler, M. J., Lehtipalo, K., Makhmutov, V., Mann, G., Mathot, S., Merikanto, J., Miettinen, P., Nenes, A., Onnela, A., Rap, A., Reddington, C. L. S., Riccobono, F., Richards, N. A. D., Rissanen, M. P., Rondo, L., Sarnela, N., Schobesberger, S., Sengupta, K., Simon, M., Sipilä, M., Smith, J. N., Stozkhov, Y., Tomé, A., Tröstl, J., Wagner, P. E., Wimmer, D., Winkler, P. M., Worsnop, D. R., and Carslaw, K. S.: 2016, Science 354, 1119

Ebisuzaki, W.: 1997, J. Clim 10, 2147 
Eddy, J. A.: 1976, Science 192(4245), 1189

Egorova, T., Schmutz, W., Rozanov, E., Shapiro, A. I., Usoskin, I. G., Beer, J., Tagirov, R. V., and Peter, T.: 2018, AEFA(submitted)

Elling, W. and Schwentek, H.: 1992, Sol. Phys. 138, 425

Elsworth, Y., Howe, R., Isaak, G. R., McLeod, C. P., Miller, B. A., New, R., Wheeler, S. J., and Gough, D. O.: 1995, Nature 376, 669

Ermolli, I., Caccin, B., Centrone, M., and Penza, V.: 2003, Mem. Soc. Astron. Italiana 74, 603

Ermolli, I., Criscuoli, S., and Giorgi, F.: 2011, Contributions of the Astronomical Observatory Skalnate Pleso 41, 73

Ermolli, I., Matthes, K., Dudok de Wit, T., Krivova, N. A., Tourpali, K., Weber, M., Unruh, Y. C., Gray, L., Langematz, U., Pilewskie, P., Rozanov, E., Schmutz, W., Shapiro, A. I., Solanki, S. K., and Woods, T. N.: 2013, Atmos. Chem. Phys. 13, 3945

Ermolli, I., Solanki, S. K., Tlatov, A. G., Krivova, N. A., Ulrich, R. K., and Singh, J.: 2009, ApJ 698, 1000

Fan, Y., Fisher, G. H., and McClymont, A. N.: 1994, ApJ 436, 907

Fasso, A., Ferrari, A., Ranft, J., and Sala, P. R.: 1993, Conf. Proc. C9309194, 493

Ferrari, A. and Sala, P. R.: 2001, Frascati Phys. Ser. 21, 31

Ferreira, S. E. S. and Potgieter, M. S.: 2004, ApJ 603, 744

Feynman, J. and Ruzmaikin, A.: 2014, J. Geophys. Res. 119(8), 6027

Field, C. V., Schmidt, G. A., Koch, D., and Salyk, C.: 2006, J. Geophys. Res. 111, D15107

Finkel, R. C. and Nishiizumi, K.: 1997, J. Geophys. Res. 102, 26

Fligge, M. and Solanki, S. K.: 2000, Geophys. Res. Lett. 27, 2157

Fligge, M., Solanki, S. K., and Beer, J.: 1999, AEEA 346, 313

Fligge, M., Solanki, S. K., and Unruh, Y. C.: 2000, AEFA 353, 380

Fligge, M., Solanki, S. K., Unruh, Y. C., Froehlich, C., and Wehrli, C.: 1998, AE्EA 335, 709

Floyd, L. E., Cook, J. W., Herring, L. C., and Crane, P. C.: 2003, Advances in Space Research 31, 2111

Fontenla, J. and Harder, G.: 2005, Mem. Soc. Astron. Italiana 76, 826 
Fontenla, J., White, O. R., Fox, P. A., Avrett, E. H., and Kurucz, R. L.: 1999, ApJ 518, 480

Fontenla, J. M., Avrett, E., Thuillier, G., and Harder, J.: 2006, ApJ 639, 441

Fontenla, J. M., Curdt, W., Haberreiter, M., Harder, J., and Tian, H.: 2009, ApJ 707, 482

Fontenla, J. M., Harder, J., Livingston, W., Snow, M., and Woods, T.: 2011, J. Geophys. Res. 116(15), D20108

Fontenla, J. M., Harder, J., Rottman, G., Woods, T. N., Lawrence, G. M., and Davis, S.: 2004, ApJ 605, L85

Forbush, S. E.: 1938, Phys. Rev. 54, 975

Forbush, S. E.: 1954, J. Geophys. Res. 59, 525

Foukal, P. and Lean, J.: 1986, ApJ 302, 826

Foukal, P., Ortiz, A., and Schnerr, R.: 2011, ApJ 733, L38

Friis-Christensen, E. and Lassen, K.: 1991, Science 254, 698

Frisius, G., Gregorius, B., Petrus, P., and Georg, P.: 1545, De radio astronomico $\mathcal{E}$ geometrico liber, Antuerpiae : apud Greg. Bontium \& Louanii : apud Petrum Phalesium

Froehlich, C. and Brusa, R.: 1981, Sol. Phys. 74, 209

Froehlich, C., Pap, J. M., and Hudson, H. S.: 1994, Sol. Phys. 152, 111

Fröhlich, C.: 2000, Space Sci. Rev. 94, 15

Fröhlich, C.: 2003, in A. Wilson (ed.), Solar Variability as an Input to the Earth's Environment, Vol. 535 of ESA Special Publication, pp 183-193

Fröhlich, C.: 2006, Space Sci. Rev. 125, 53

Fröhlich, C.: 2009, AEFA 501, L27

Fröhlich, C.: 2012, Surveys in Geophysics 33, 453

Garcia, A. and Mouradian, Z.: 1998, Sol. Phys. 180, 495

Garcia-Munoz, M., Mason, G. M., and Simpson, J. A.: 1975, ApJ 202, 265

Garcon, V. C. and Minster, J.-F.: 1988, Tellus Series B Chemical and Physical Meteoro$\log y B$ 40, 161

Geiger, H. and Müller, W.: 1928, Naturwissenschaften 16(31), 617

Genevey, A., Gallet, Y., Constable, C. G., Korte, M., and Hulot, G.: 2008, Geochem. Geophys. Geosyst. 9, Q04038 
Gillett, N. P. and Thompson, D. W. J.: 2003, Science 302, 273

Glatzmaier, G. A., Ogden, D. E., and Clune, T. L.: 2004, Washington DC American Geophysical Union Geophysical Monograph Series 150, 13

Glatzmaier, G. A. and Roberts, P. H.: 1995, Phys. Earth Planet. Inter 91, 63

Gleeson, L. J. and Axford, W. I.: 1968, ApJ 154, 1011

Gleissberg, W.: 1939, The Observatory 62, 158

Gleissberg, W.: 1960, Naturwissenschaften 47, 197

Godwin, H.: 1962, Nature 195, 984

Goel, P. S., Kharkar, D. P., Lal, D., Narsappaya, N., Peters, B., and Yatirajam, V.: 1957, Deep Sea Research 4, 202

Goode, P. R.: 1995, in Helioseismology, Vol. 376 of ESA Special Publication, p. 121

Goorley, T., James, M., Booth, T., Brown, F., Bull, J., Cox, L. J., Durkee, J., Elson, J., Fensin, M., Forster, R. A., Hendricks, J., Hughes, H. G., Johns, R., Kiedrowski, B., Martz, R., Mashnik, S., McKinney, G., Pelowitz, D., Prael, R., Sweezy, J., Waters, L., Wilcox, T., and Zukaitis, T.: 2012, Nuclear Technology 180(3), 298

Gordon, H., Kirkby, J., Baltensperger, U., Bianchi, F., Breitenlechner, M., Curtius, J., Dias, A., Dommen, J., Donahue, N. M., Dunne, E. M., Duplissy, J., Ehrhart, S., Flagan, R. C., Frege, C., Fuchs, C., Hansel, A., Hoyle, C. R., Kulmala, M., Kürten, A., Lehtipalo, K., Makhmutov, V., Molteni, U., Rissanen, M. P., Stozkhov, Y., Tröstl, J., Tsagkogeorgas, G., Wagner, R., Williamson, C., Wimmer, D., Winkler, P. M., Yan, C., and Carslaw, K. S.: 2017, J. Geophys. Res. 122, 8739

Goslar, T.: 2001, Radiocarbon 43(2B), 743

Goslar, T.: 2003, PAGES news 11, 12

Gough, D. and Toomre, J.: 1991, ARAEFA 29, 627

Gradstein, F. M., Ogg, J. G., and Smith, A. G.: 2005, A Geologic Time Scale 2004, Cambridge, UK: Cambridge University Press

Gray, L. J., Beer, J., Geller, M., Haigh, J. D., Lockwood, M., Matthes, K., Cubasch, U., Fleitmann, D., Harrison, G., Hood, L., Luterbacher, J., Meehl, G. A., Shindell, D., van Geel, B., and White, W.: 2010, Rev. Geophys. 48, RG4001

Gubbins, D.: 1994, Rev. of Geophys. 32, 61

Haberreiter, M., Krivova, N. A., Schmutz, W., and Wenzler, T.: 2005, Advances in Space Research 35, 365

Haberreiter, M., Schmutz, W., and Hubeny, I.: 2008, AEFA 492, 833 
Hagenaar, H. J.: 2001, ApJ 555, 448

Hagenaar, H. J., DeRosa, M. L., and Schrijver, C. J.: 2008, ApJ 678, 541

Hagenaar, H. J., Schrijver, C. J., and Title, A. M.: 2003, ApJ 584, 1107

Haigh, J. D.: 1994, Nature 370, 544

Haigh, J. D.: 1996, Science 272, 981

Haigh, J. D.: 1999, Jour. of Atmos. and Sol.-Terr. Phys. 61, 63

Haigh, J. D.: 2001, Science 294, 2109

Haigh, J. D.: 2003, Philos. Trans. R. Soc. Lond. A 361, 95

Haigh, J. D.: 2007, Living Rev. SoL. Phys. 4, 2

Hale, G. E. and Nicholson, S. B.: 1925, ApJ 62, 270

Hansen, J. E.: 2000, Space Sci. Rev. 94, 349

Harder, J., Lawrence, G., Fontenla, J., Rottman, G., and Woods, T.: 2005, Sol. Phys. 230, 141

Harder, J. W., Fontenla, J. M., Pilewskie, P., Richard, E. C., and Woods, T. N.: 2009, Geophys. Res. Lett. 36, L07801

Harvey, K. L.: 1992, in The Solar Cycle, Vol. 27 of ASP Conf. Ser., p. 335

Harvey, K. L.: 1993, Ph.D. thesis, Univ. Utrecht, Netherland

Harvey, K. L.: 1994, in R. J. Rutten and C. J. Schrijver (eds.), NATO Advanced Science Institutes (ASI) Series C, Vol. 433 of NATO Advanced Science Institutes (ASI) Series C, p. 347

Harvey, K. L. and Martin, S. F.: 1973, Sol. Phys. 32(2), 389

Hathaway, D. H.: 2015, Living Rev. Sol. Phys. 12, 4

Hathaway, D. H., Wilson, R. M., and Reichmann, E. J.: 1994, Sol. Phys. 151, 177

Hathaway, D. H., Wilson, R. M., and Reichmann, E. J.: 2002, Sol. Phys. 211(1-2), 357

Heath, D. F. and Schlesinger, B. M.: 1986, J. Geophys. Res. 91, 8672

Heikkilä, U., Beer, J., Abreu, J. A., and Steinhilber, F.: 2013, Space Sci. Rev. 176, 321

Heikkilä, U., Beer, J., and Feichter, J.: 2009, Atmos. Chem. Phys. 9, 515

Herbst, K., Kopp, A., Heber, B., Steinhilber, F., Fichtner, H., Scherer, K., and Matthiä, D.: 2010, J. Geophys. Res. 115, D00I20

Herbst, K., Muscheler, R., and Heber, B.: 2017, J. Geophys. Res. 122, 23 
Herschel, W.: 1801, Philosophical Transactions of the Royal Society of London Series I 91, 265

Hess, V. F.: 1912, Phys. Z. 13, 1084

Heyvaerts, J. and Priest, E. R.: 1983, AEFA 117, 220

Hoek, W. Z. and Bohncke, S. J. P.: 2001, Quat. Sci. Rev. 20, 1251

Horiuchi, K., Ohta, A., Uchida, T., Matsuzaki, H., Shibata, Y., and Motoyama, H.: 2007, Nuclear Instruments and Methods in Physics Research B 259, 584

Horiuchi, K., Uchida, T., Sakamoto, Y., Ohta, A., Matsuzaki, H., Shibata, Y., and Motoyama, H.: 2008, Quaternary Geochronology 3, 253

Howe, R., Christensen-Dalsgaard, J., Hill, F., Komm, R. W., Larsen, R. M., Schou, J., Thompson, M. J., and Toomre, J.: 2000, Science 287, 2456

Hoyt, D. V. and Schatten, K. H.: 1998, Sol. Phys. 181, 491

Hudson, H. S.: 1988, ARAEFA 26, 473

Hudson, H. S.: 1991, Sol. Phys. 133, 357

Hudson, H. S., Silva, S., Woodard, M., and Willson, R. C.: 1982, Sol. Phys. 76, 211

Hughen, K., Southon, J., Lehman, S., Bertrand, C., and Turnbull, J.: 2006, Quaternary Science Reviews 25, 3216

Hultqvist, M., Lazzeroni, M., Botvina, A., Gudowska, I., Sobolevsky, N., and Brahme, A.: 2012, Physics in Medicine and Biology 57, 4369

Hvidberg, C. S., Steffensen, J. P., Clausen, H. B., Shoji, H., and Kipfstuhl, J.: 2002, Annals of Glaciology 35, 5

Inceoglu, F., Simoniello, R., Knudsen, M. F., Karoff, C., Olsen, J., Turck-Chiéze, S., and Jacobsen, B. H.: 2015, AEFA 577, A20

Jiang, J., Cameron, R. H., Schmitt, D., and Schüssler, M.: 2011a, AEFA 528, A82

Jiang, J., Cameron, R. H., Schmitt, D., and Schüssler, M.: 2011b, AEFA 528, A83

Jiang, J., Hathaway, D. H., Cameron, R. H., Solanki, S. K., Gizon, L., and Upton, L.: 2014, Space Sci. Rev. 186(1), 491

Johnsen, S. J., Dahl-Jensen, D., Dansgaard, W., and Gundestrup, N.: 1995, Tellus Series $B$ Chemical and Physical Meteorology B 47, 624

Johnsen, S. J., Dahl-Jensen, D., Gundestrup, N., Steffensen, J. P., Clausen, H. B., Miller, H., Masson-Delmotte, V., Sveinbjörnsdottir, A. E., and White, J.: 2001, J. Quat. Sci 16, 299

Joos, F.: 1994, Nature 370, 181 
Jungclaus, J. H., Bard, E., Baroni, M., Braconnot, P., Cao, J., Chini, L. P., Egorova, T., Evans, M., González-Rouco, J. F., Goosse, H., Hurtt, G. C., Joos, F., Kaplan, J. O., Khodri, M., Klein Goldewijk, K., Krivova, N. A., LeGrande, A. N., Lorenz, S. J., Luterbacher, J., Man, W., Meinshausen, M., Moberg, A., Nehrbass-Ahles, C., Otto-Bliesner, B. I., Phipps, S. J., Pongratz, J., Rozanov, E., Schmidt, G. A., Schmidt, H., Schmutz, W., Schurer, A., Shapiro, A. I., Sigl, M., Smerdon, J. E., Solanki, S. K., Timmreck, C., Toohey, M., Usoskin, I. G., Wagner, S., Wu, C.-J., Yeo, K. L., Zanchettin, D., Zhang, Q., and Zorita, E.: 2016, Geosci. Model Dev. Disc. 10(11), 4005

Jungclaus, J. H., Lorenz, S. J., Timmreck, C., Reick, C. H., Brovkin, V., Six, K., Segschneider, J., Giorgetta, M. A., Crowley, T. J., Pongratz, J., Krivova, N. A., Vieira, L. E. A., Solanki, S. K., Klocke, D., Botzet, M., Esch, M., Gayler, V., Haak, H., Raddatz, T. J., Roeckner, E., Schnur, R., Widmann, H., Claussen, M., Stevens, B., and Marotzke, J.: 2010, Clim. Past 6(5), 723

Kamen, M. D.: 1963, Science 140, 584

Kane, R. P.: 2008, Sol. Phys. 248, 203

Käpylä, M. J., Käpylä, P. J., Olspert, N., Brandenburg, A., Warnecke, J., Karak, B. B., and Pelt, J.: 2016, AEFA 589, A56

Karak, B. B. and Choudhuri, A. R.: 2010, Astrophysics and Space Science Proceedings 19, 402

Keeling, C. D.: 1979, Environment International 2(4), 229

Keil, S. L., Henry, T. W., and Fleck, B.: 1998, in K. S. Balasubramaniam, J. Harvey, and D. Rabin (eds.), Synoptic Solar Physics, Vol. 140 of Astronomical Society of the Pacific Conference Series, p. 301

Keller, C. U., Schüssler, M., Vögler, A., and Zakharov, V.: 2004, ApJ 607, L59

Keppens, R. and Martínez Pillet, V.: 1996, AEFA 316, 229

Kirkby, J., Curtius, J., Almeida, J., Dunne, E., Duplissy, J., Ehrhart, S., Franchin, A., Gagné, S., Ickes, L., Kürten, A., Kupc, A., Metzger, A., Riccobono, F., Rondo, L., Schobesberger, S., Tsagkogeorgas, G., Wimmer, D., Amorim, A., Bianchi, F., Breitenlechner, M., David, A., Dommen, J., Downard, A., Ehn, M., Flagan, R. C., Haider, S., Hansel, A., Hauser, D., Jud, W., Junninen, H., Kreissl, F., Kvashin, A., Laaksonen, A., Lehtipalo, K., Lima, J., Lovejoy, E. R., Makhmutov, V., Mathot, S., Mikkilä, J., Minginette, P., Mogo, S., Nieminen, T., Onnela, A., Pereira, P., Petäjä, T., Schnitzhofer, R., Seinfeld, J. H., Sipilä, M., Stozhkov, Y., Stratmann, F., Tomé, A., Vanhanen, J., Viisanen, Y., Vrtala, A., Wagner, P. E., Walther, H., Weingartner, E., Wex, H., Winkler, P. M., Carslaw, K. S., Worsnop, D. R., Baltensperger, U., and Kulmala, M.: 2011, Nature 476, 429

Knudsen, M. F., Riisager, P., Donadini, F., Snowball, I., Muscheler, R., Korhonen, K., and Pesonen, L. J.: 2008, Earth Planet. Sci. Lett. 272, 319 
Knudsen, M. F., Riisager, P., Jacobsen, B. H., Muscheler, R., Snowball, I., and Seidenkrantz, M.-S.: 2009, Geophys. Res. Lett. 36, L16701

Kocharov, G. E., Blinov, A. V., Konstantinov, A. N., and Levchenko, V. A.: 1989, Geomagn. Aeron. 28, 706

Kocharov, G. E., Ostryakov, V. M., Peristykh, A. N., and Vasil'Ev, V. A.: 1995, Sol. Phys. 159, 381

Kodera, K. and Kuroda, Y.: 2002, J. Geophys. Res. 107, 4749

Kopp, G.: 2014, J. Space Weather Space Clim. 4, A14

Kopp, G.: 2016, J. Space Weather Space Clim. 6(27), A30

Kopp, G., Heuerman, K., Harber, D., and Drake, G.: 2007, in Earth Observing Systems XII, Vol. 6677 of Proc. SPIE, p. 667709

Kopp, G., Heuerman, K., and Lawrence, G.: 2005a, Sol. Phys. 230, 111

Kopp, G., Krivova, N. A., Wu, C.-J., and Lean, J.: 2016, Sol. Phys. 291, 2951

Kopp, G. and Lawrence, G.: 2005, Sol. Phys. 230, 91

Kopp, G., Lawrence, G., and Rottman, G.: 2005b, Sol. Phys. 230, 129

Korschinek, G., Bergmaier, A., Faestermann, T., Gerstmann, U. C., Knie, K., Rugel, G., Wallner, A., Dillmann, I., Dollinger, G., von Gostomski, C. L., Kossert, K., Maiti., M., Poutivtsev, M., and Remmert, A.: 2010, Nuclear Instruments and Methods in Physics Research Section B: Beam Interactions with Materials and Atoms 268(2), 187

Korte, M. and Constable, C. G.: 2005, Earth Planet. Sci. Lett. 236, 348

Korte, M. and Constable, C. G.: 2006, Geochemistry, Geophysics, Geosystems 7, Q09004 Kovaltsov, G. A., Mishev, A., and Usoskin, I. G.: 2012, Earth Planet. Sci. Lett. 337, 114

Kovaltsov, G. A. and Usoskin, I. G.: 2010, Earth Planet. Sci. Lett. 291(1-4), 182

Kren, A. C., Pilewskie, P., and Coddington, O.: 2017, J. Space Weather and Space Clim. 7(27), A10

Krivova, N. A., Balmaceda, L., and Solanki, S. K.: 2007, AEFA 467, 335

Krivova, N. A. and Solanki, S. K.: 2004, AEA A 417, 1125

Krivova, N. A. and Solanki, S. K.: 2005, Adv. Space Res. 35, 361

Krivova, N. A., Solanki, S. K., Fligge, M., and Unruh, Y. C.: 2003, AEA 399, L1

Krivova, N. A., Solanki, S. K., and Floyd, L.: 2006, AEFA 452, 631

Krivova, N. A., Vieira, L. E. A., and Solanki, S. K.: 2010, J. Geophys. Res. 115, A12112 
Kromer, B.: 2009, Dendrochronologia 27(1), 15

Küker, M., Arlt, R., and Rüdiger, G.: 1999, AEFA 343, 977

Kurucz, R. L.: 1993, Smithsonian Astrophysical Observatory: Cambridge, MA Kurucz CD-ROM No. 13

Kurucz, R. L.: 2005, Memorie della Societa Astronomica Italiana Supplementi 8, 14

Lagg, A., Solanki, S. K., Riethmüller, T. L., Martínez Pillet, V., Schüssler, M., Hirzberger, J., Feller, A., Borrero, J. M., Schmidt, W., del Toro Iniesta, J. C., Bonet, J. A., Barthol, P., Berkefeld, T., Domingo, V., Gandorfer, A., Knölker, M., and Title, A. M.: 2010, ApJ 723, L164

Lal, D.: 1988, Ann. Rev. Earth Planet. Sci. Lett. 16, 355

Lal, D. and Jull, A. J. T.: 2001, Radiocarbon 43(2B), 731

Lal, D. and Peters, B.: 1967, Handbuch der Physik 46, 551

Lal, D. and Suess, H. E.: 1968, Annual Review of Nuclear and Particle Science 18, 407

Lange, I. and Forbush, S. E.: 1948, Cosmic-Ray Results From Huancayo Observatory, Peru, June1936-December, 1946, Carnegie Institution of Washington, Washington, D. $\mathrm{C}$

Larkin, A., Haigh, J. D., and Djavidnia, S.: 2000, Space Sci. Rev. 94, 199

Le Treut, H.: 2012, Surv. in Geo. 33, 723

Lean, J.: 2000, Geophys. Res. Lett. 27, 2425

Lean, J. L., Rottman, G. J., Kyle, H. L., Woods, T. N., Hickey, J. R., and Puga, L. C.: 1997, J. Geophys. Res. 102, 29939

Leighton, R. B.: 1969, ApJ 156, 1

Leussu, R., Usoskin, I. G., Arlt, R., and Mursula, K.: 2013, AEFA 559, A28

Libby, W. F.: 1952, Science 116, 673

Libby, W. F., Anderson, E. C., and Arnold, J. R.: 1949, Science 109, 227

Licht, A., Hulot, G., Gallet, Y., and Thébault, E.: 2013, Phys. Earth Planet. Inter 224, 38

Livingston, W.: 2002, Sol. Phys. 207, 41

Livingston, W. C., Harvey, J., Pierce, A. K., Schrage, D., Gillespie, B., Simmons, J., and Slaughter, C.: 1976, Appl. Opt. 15, 33

Lockwood, M., Nevanlinna, H., Barnard, L., Owens, M. J., Harrison, R. G., Rouillard, A. P., and Scott, C. J.: 2014a, Annal. Geophys. 32, 383 
Lockwood, M., Owens, M. J., and Barnard, L.: 2014b, J. Geophys. Res. 119, 5172

Lockwood, M., Owens, M. J., and Barnard, L.: 2014c, J. Geophys. Res. 119, 5183

Lockwood, M., Owens, M. J., Barnard, L., and Usoskin, I. G.: 2016, ApJ 824, 54

Lockwood, M., Rouillard, A. P., and Finch, I. D.: 2009, ApJ 700, 937

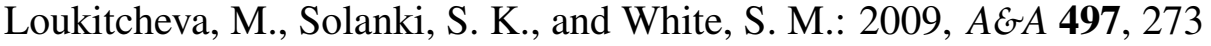

Mackay, D. H. and Yeates, A. R.: 2012, Liv. Rev. in Sol. Phys. 9, 6

Mann, M. E.: 2002, Encyclopedia of global environmental change 1, 504

Marmatsouri, L., Vassilaki, A., Mavromichalaki, H., and Petropoulos, B.: 1995, Adv. Space Res. 16, 245

Marsh, N. D. and Svensmark, H.: 2000a, Space Sci. Rev. 94, 215

Marsh, N. D. and Svensmark, H.: 2000b, Phys. Rev. Lett. 85, 5004

Martin, S. F. and Harvey, K. H.: 1979, Sol. Phys. 64, 93

Masarik, J. and Beer, J.: 1999, J. Geophys. Res. 104(D10), 12099

Masarik, J. and Beer, J.: 2009, J. Geophys. Res. 114(13), D11103

Masarik, J. and Reedy, R. C.: 1995, Earth Planet. Sci. Lett. 136, 381

Mathew, S. K., Martínez Pillet, V., Solanki, S. K., and Krivova, N. A.: 2007, AEFA 465, 291

Matthes, K., Funke, B., Andersson, M. E., Barnard, L., Beer, J., Charbonneau, P., Clilverd, M. A., Dudok de Wit, T., Haberreiter, M., Hendry, A., Jackman, C. H., Kretzschmar, M., Kruschke, T., Kunze, M., Langematz, U., Marsh, D. R., Maycock, A. C., Misios, S., Rodger, C. J., Scaife, A. A., Seppälä, A., Shangguan, M., Sinnhuber, M., Tourpali, K., Usoskin, I. G., van de Kamp, M., Verronen, P. T., and Versick, S.: 2017, Geoscientific Model Development 10(6), 2247

Matthes, K., Kuroda, Y., Kodera, K., and Langematz, U.: 2006, J. Geophys. Res. 111, D06108

Maunder, E. W.: 1903, The Observatory 26, 329

Mavromichalaki, H., Gerontidou, M., Paschalis, P., and Paouris, E.: 2016, ArXiv e-prints

McCracken, K. G. and Beer, J.: 2007, J. Geophys. Res. 112, A10101

McCracken, K. G. and Beer, J.: 2015, Sol. Phys. 290, 3051

McCracken, K. G., McDonald, F. B., Beer, J., Raisbeck, G., and Yiou, F.: 2004, J. Geophys. Res. 109, A12103 
McDonald, F. B.: 1998, Space Sci. Rev. 83, 33

McDonald, F. B., Webber, W. R., and Reames, D. V.: 2010, Geophys. Res. Lett. 37, L18101

McElhinny, M. W. and McFadden, P. L.: 2000, Paleomagnetism: Continents and Oceans, International geophysics series, Academic Press

McElhinny, M. W. and Senanayake, W. E.: 1982, Journal of Geomagnetism and Geoelectricity 34, 39

McHargue, L. R. and Damon, P. E.: 1991, Rev. Geophys. 29(2), 141

McKinnon, J. A. and Waldmeier, M.: 1987, Sunspot numbers, 1610-1985 : based on "The sunspot activity in the years 1610-1960", Report UAG-95, WDC-A, Boulder, CO.,

Meehl, G. A., Arblaster, J. M., Matthes, K., Sassi, F., and van Loon, H.: 2009, Science 325, 1114

Mekhaldi, F., Muscheler, R., Adolphi, F., Aldahan, A., Beer, J., McConnell, J. R., Possnert, G., Sigl, M., Svensson, A., Synal, H.-A., Welten, K. C., and Woodruff, T. E.: 2015, Nature Communication 6, 8611

Merrill, R. T. and McFadden, P. L.: 1999, Rev. of Geophys. 37, 201

Meunier, N.: 2003, AEFA 405, 1107

Millikan, R. A. and Bowen, I. S.: 1931, Nature 128, 582

Miyahara, H., Masuda, K., Muraki, Y., Furuzawa, H., Menjo, H., and Nakamura, T.: 2004, Sol. Phys. 224, 317

Miyake, F., Jull, A. J. T., Panyushkina, I. P., Wacker, L., Salzer, M., Baisan, C. H., Lange, T., Cruz, R., Masuda, K., and Nakamura, T.: 2017, Proceedings of the National Academy of Science 114, 881

Miyake, F., Masuda, K., and Nakamura, T.: 2013, Nature Communication 4, 1748

Miyake, F., Nagaya, K., Masuda, K., and Nakamura, T.: 2012, Nature 486, 240

Moraal, H. and Stoker, P. H.: 2010, J. Geophys. Res. 115(A12), A12109

Morrill, J. S., Floyd, L., and McMullin, D.: 2014, Sol. Phys. 289, 3641

Morrill, J. S., Floyd, L., Ulrich, R., Weaver, S., and McMullin, D.: 2011, Sol. Phys. 270, 109

Moss, D., Sokoloff, D., Usoskin, I., and Tutubalin, V.: 2008, Sol. Phys. 250, 221

Mowlavi, N., Eggenberger, P., Meynet, G., Ekström, S., Georgy, C., Maeder, A., Charbonnel, C., and Eyer, L.: 2012, A\& $A$ 541, A41 
Munro, R., Anderson, C., Callies, J., and et al.: 2006, in Atmospheric Science Conference, Vol. 628 of ESA Special Publication, p. 94

Mursula, K., Usoskin, I. G., and Kovaltsov, G. A.: 2001, Sol. Phys. 198, 51

Muscheler, R., Adolphi, F., and Svensson, A.: 2014, Earth Planet. Sci. Lett. 394, 209

Muscheler, R., Beer, J., Kubik, P. W., and Synal, H.-A.: 2005, Quaternary Science Reviews 24, 1849

Muscheler, R., Beer, J., Wagner, G., Laj, C., Kissel, C., Raisbeck, G. M., Yiou, F., and Kubik, P. W.: 2004, Earth Planet. Sci. Lett. 219, 325

Muscheler, R., Joos, F., Beer, J., Müller, S. A., Vonmoos, M., and Snowball, I.: 2007, Quaternary Science Reviews 26, 82

Nagashima, K. and Morishita, I.: 1979, International Cosmic Ray Conference 3, 313

Neher, H. V.: 1971, J. Geophys. Res. 76, 1637

Neher, H. V., Peterson, V. Z., and Stern, E. A.: 1953, Phys. Rev. 90, 655

Ness, N. F.: 1969, Rev. of Geo. and Sp. Phys. 7, 97

Ney, E. P.: 1959, Nature 183, 451

Nilsson, A., Holme, R., Korte, M., Suttie, N., and Hill, M.: 2014, Geophysical Journal International 198, 229

Nordemann, D. J. R.: 1992, Sol. Phys. 141, 199

Nordlund, Å., Stein, R. F., and Asplund, M.: 2009, Living Rev. Sol. Phys. 6, 2

O’brien, K.: 1979, J. Geophys. Res. 84, 423

Oeschger, H., Siegenthaler, U., U., S., and Gugelmann, A.: 1975, Tellus 27(2), 168

Olson, P., Christensen, U., and Glatzmaier, G. A.: 1999, J. Geophys. Res. 104, 10

Ortiz, A., Solanki, S. K., Domingo, V., M., F., and Sanahuja, B.: 2002, AEFA 388, 1036

Oster, L., Schatten, K. H., and Sofia, S.: 1982, ApJ 256, 768

Owens, M. J. and Lockwood, M.: 2012, J. Geophys. Res. 117, A04102

Owens, M. J., Usoskin, I. G., and Lockwood, M.: 2012, Geophys. Res. Lett. 39, L19102

Paillard, D.: 2001, Rev. of Geophys. 39, 325

Parker, E. N.: 1963, Interplanetary dynamical processes.

Parker, E. N.: 1965, Planet. Space Sci. 13, 9

Parker, E. N.: 1972, ApJ 174, 499 
Parker, E. N.: 1979, ApJ 230, 905

Parker, E. N.: 1983, in R. L. Carovillano and J. M. Forbes (eds.), Solar-Terrestrial Physics: Principles and Theoretical Foundations, Vol. 104 of Astrophysics and Space Science Library, pp 129-154

Parker, E. N.: 1988, ApJ 330, 474

Parnell, C. E. and De Moortel, I.: 2012, Philosophical Transactions of the Royal Society of London Series A 370, 3217

Pavlov, A. K., Blinov, A. V., Frolov, D. A., Konstantinov, A. N., Ostryakov, V. M., and Vasilyev, G. I.: 2017, J. Atmospheric Sol.-Terr. Phys. 164, 308

Pavón-Carrasco, F. J., Osete, M. L., Torta, J. M., and De Santis, A.: 2014, Earth Planet. Sci. Lett. 388, 98

Pedro, J. B., Heikkilä, U. E., Klekociuk, A., Smith, A. M., van Ommen, T. D., and Curran, M. A. J.: 2011, J. Geophys. Res. 116(15), D23120

Penza, V., Caccin, B., Ermolli, I., Centrone, M., and Gomez, M. T.: 2003, in A. Wilson (ed.), Solar Variability as an Input to the Earth's Environment, Vol. 535 of ESA Special Publication, pp 299-302

Poluianov, S. V., Kovaltsov, G. A., Mishev, A. L., and Usoskin, I. G.: 2016, J. Geophys. Res. 121, 8125

Potgieter, M. S.: 1998, Space Sci. Rev. 83, 147

Preminger, D. G., Walton, S. R., and Chapman, G. A.: 2002, J. Geophys. Res. 107, 1354

Priest, E. R., Heyvaerts, J. F., and Title, A. M.: 2002, ApJ 576, 533

Protheroe, R. J. and Szabo, A. P.: 1992, Phys. Rev. Lett. 69, 2885

Pshenichnov, I., Botvina, A., Mishustin, I., and Greiner, W.: 2010, Nuclear Instruments and Methods in Physics Research B 268, 604

Raisbeck, G. M., Yiou, F., Fruneau, M., Loiseaux, J. M., Lieuvin, M., Ravel, J. C., and Lorius, C.: 1981, Nature 292, 825

Raisbeck, G. M., Yiou, F., Jouzel, J., and Petit, J. R.: 1990, Philosophical Transactions of the Royal Society of London Series A 330, 463

Rasmussen, S. O., Bigler, M., Blockley, S. P., Blunier, T., Buchardt, S. L., Clausen, H. B., Cvijanovic, I., Dahl-Jensen, D., Johnsen, S. J., Fischer, H., Gkinis, V., Guillevic, M., Hoek, W. Z., Lowe, J. J., Pedro, J. B., Popp, T., Seierstad, I. K., Steffensen, J. P., Svensson, A. M., Vallelonga, P., Vinther, B. M., Walker, M. J. C., Wheatley, J. J., and Winstrup, M.: 2014, Quat. Sci. Rev. 106, 14

Reames, D. V.: 1999, Space Sci. Rev. 90, 413 
Reeves, E. M., Timothy, J. G., and Huber, M. C. E.: 1977, Appl. Opt. 16, 837

Reid, G. C.: 1987, Nature 329, 142

Reimer, P. J., Baillie, M. G. L., Bard, E., Bayliss, A., Beck, J. W., Blackwell, P. G., Ramsey, C. B., Buck, C. E., Burr, G. S., Edwards, R. L., Friedrich, M., Grootes, P. M., Guilderson, T. P., Hajdas, I., Heaton, T. J., Hogg, A. G., Hughen, K. A., Kaiser, K. F., Kromer, B., McCormac, F. G., Manning, S. W., Reimer, R. W., Richards, D. A., Southon, J. R., Talamo, S., Turney, C. S. M., van der Plicht, J., and Weyhenmeyer, C. E.: 2009, Radiocarbon 51(4), 1111

Rempel, M. and Schlichenmaier, R.: 2011, Liv. Rev. in Sol. Phys. 8, 3

Rezaei, R., Steiner, O., Wedemeyer-Böhm, S., Schlichenmaier, R., Schmidt, W., and Lites, B. W.: 2007, AEFA 476(3), L33

Ribes, J. C. and Nesme-Ribes, E.: 1993, A\& $A$ 276, 549

Roth, R. and Joos, F.: 2013, Clim. Past 9(4), 1879

Rottman, G. J.: 1988, Advances in Space Research 8, 53

Rottman, G. J., Woods, T. N., and Sparn, T. P.: 1993, J. Geophys. Res. 98, 10

Rüdiger, G. and Hollerbach, R.: 2004, The magnetic universe : geophysical and astrophysical dynamo theory, Wiley-VCH

Ruth, U., Barnola, J.-M., Beer, J., Bigler, M., Blunier, T., Castellano, E., Fischer, H., Fundel, F., Huybrechts, P., Kaufmann, P., Kipfstuhl, S., Lambrecht, A., Morganti, A., Oerter, H., Parrenin, F., Rybak, O., Severi, M., Udisti, R., Wilhelms, F., and Wolff, E.: 2007, Clim. Past 3, 475

Sackmann, I. J., Boothroyd, A. I., and Kraemer, K. E.: 1993, ApJ 418, 457

Scaife, A. A., Knight, J. R., Vallis, G. K., and Folland, C. K.: 2005, Geophys. Res. Lett. 32, L18715

Scharmer, G. B., Bjelksjo, K., Korhonen, T. K., Lindberg, B., and Petterson, B.: 2003, in S. L. Keil and S. V. Avakyan (eds.), Innovative Telescopes and Instrumentation for Solar Astrophysics, Vol. 4853 of Proc. SPIE, pp 341-350

Schmidt, G. A., Jungclaus, J. H., Ammann, C. M., Bard, E., Braconnot, P. C. T. J. D. G., Crowley, T. J., Delaygue, G., Joos, F., Krivova, N. A., Muscheler, R., et al.: 2011, Geoscientific Model Development 4(1), 33

Schmitt, D., Sch'ussler, M., and Ferriz-Mas, A.: 1996, AEFA 311, L1

Schmutz, W., Fehlmann, A., Finsterle, W., Kopp, G., and Thuillier, G.: 2013, in American Institute of Physics Conference Series, Vol. 1531 of American Institute of Physics Conference Series, pp 624-627 
Schmutz, W., Fehlmann, A., Hülsen, G., Meindl, P., Winkler, R., Thuillier, G., Blattner, P., Buisson, F., Egorova, T., Finsterle, W., Fox, N., Gröbner, J., Hochedez, J.-F., Koller, S., Meftah, M., Meisonnier, M., Nyeki, S., Pfiffner, D., Roth, H., Rozanov, E., Spescha, M., Wehrli, C., Werner, L., and Wyss, J. U.: 2009, Metrologia 46, S202

Schneider, S. H. and Mass, C.: 1975, Science 190, 741

Schou, J., Antia, H. M., Basu, S., Bogart, R. S., Bush, R. I., Chitre, S. M., ChristensenDalsgaard, J., Di Mauro, M. P., Dziembowski, W. A., Eff-Darwich, A., Gough, D. O., Haber, D. A., Hoeksema, J. T., Howe, R., Korzennik, S. G., Kosovichev, A. G., Larsen, R. M., Pijpers, F. P., Scherrer, P. H., Sekii, T., Tarbell, T. D., Title, A. M., Thompson, M. J., and Toomre, J.: 1998, ApJ 505, 390

Schrijver, C. J., Cote, J., Zwaan, C., and Saar, S. H.: 1989, ApJ 337, 964

Schrijver, C. J. and Harvey, K. L.: 1994, Sol. Phys. 150, 1

Schüssler, M. and Baumann, I.: 2006, AEFA 459, 945

Schwabe, H.: 1844, Astronomische Nachrichten 21, 233

Schwarzschild, M.: 1948, ApJ 107, 1

Seierstad, I. K., Abbott, P. M., Bigler, M., Blunier, T., Bourne, A. J., Brook, E., Buchardt, S. L., Buizert, C., Clausen, H. B., Cook, E., Dahl-Jensen, D., Davies, S. M., Guillevic, M., Johnsen, S. J., Pedersen, D. S., Popp, T. J., Rasmussen, S. O., Severinghaus, J. P., Svensson, A., and Vinther, B. M.: 2014, Quat. Sci. Rev. 106, 29

Sekido, Y., Masuda, T., Yoshida, S., and Wada, M.: 1951, Phys. Rev. 83, 658

Seleznyov, A. D., Solanki, S. K., and Krivova, N. A.: 2011, AE\&A 532, A108

Selkin, P. A. and Tauxe, L.: 2000, Philosophical Transactions of the Royal Society of London A 358(1768), 1065

Shapiro, A., Schmutz, W., Thuillier, G., Schoell, M., Haberreiter, M., and Rozanov, E.: 2010, in 38th COSPAR Scientific Assembly, Vol. 38 of COSPAR Meeting, p. 7

Shapiro, A. I., Schmutz, W., Dominique, M., and Shapiro, A. V.: 2013, Sol. Phys. 286, 271

Shapiro, A. I., Schmutz, W., Rozanov, E., Schoell, M., Haberreiter, M., Shapiro, A. V., and Nyeki, S.: 2011, AEFA 529, A67

Shapiro, A. I., Solanki, S. K., Krivova, N. A., Cameron, R. H., Yeo, K. L., and Schmutz, W. K.: 2017, Nature Astronomy 1, 612

Shaviv, N. J.: 2002, Phys. Rev. Lett 89(5), 051102

Shindell, D., Rind, D., Balachandran, N., Lean, J., and Lonergan, P.: 1999, Science 284, 305 
Siegenthaler, U., Heimann, M., and Oeschger, H.: 1980, Radiocarbon 22(2), 177

Sigl, M., Winstrup, M., McConnell, J. R., Welten, K. C., Plunkett, G., Ludlow, F., Büntgen, U., Caffee, M., Chellman, N., Dahl-Jensen, D., Fischer, H., Kipfstuhl, S., Kostick, C., Maselli, O. J., Mekhaldi, F., Mulvaney, R., Muscheler, R., Pasteris, D. R., Pilcher, J. R., Salzer, M., Schüpbach, S., Steffensen, J. P., Vinther, B. M., and Woodruff, T. E.: 2015, Nature 523, 543

Simpson, J. A.: 1953, Evidence for a Solar Cosmic-Ray Component, pp 715-721

Simpson, J. A.: 2000, Space Sci. Rev. 93, 11

Singer, B. S. and Pringle, M. S.: 1996, Earth Planet. Sci. Lett. 139, 47

Skumanich, A., Smythe, C., and Frazier, E. N.: 1975, ApJ 200, 747

Skupin, J., Noël, S., Wuttke, M. W., Gottwald, M., Bovensmann, H., Weber, M., and Burrows, J. P.: 2005, Advances in Space Research 35, 370

Smart, D. F. and Shea, M. A.: 1967, J. Geophys. Res. 72(13), 3447

Smart, D. F. and Shea, M. A.: 1985, Handbook of Geophysics and the Space Environment. Air Force Geophysics Laboratory 6, 6

Smith, E. V. P. and Gottlieb, D. M.: 1974, Space Sci. Rev. 16, 771

Smith, E. V. P. and Gottlieb, D. M.: 1975, NASA Special Publication 366

Snow, M., McClintock, W. E., Rottman, G., and Woods, T. N.: 2005, Sol. Phys. 230, 295

Sokoloff, D. and Nesme-Ribes, E.: 1994, AEFA 288, 293

Solanki, S. K.: 1999, in C. J. Butler and J. G. Doyle (eds.), Solar and Stellar Activity: Similarities and Differences, Vol. 158 of Astronomical Society of the Pacific Conference Series, p. 109

Solanki, S. K.: 2003, AEFA Rev. 11, 153

Solanki, S. K., Barthol, P., Danilovic, S., Feller, A., Gandorfer, A., Hirzberger, J., Riethmüller, T. L., Schüssler, M., Bonet, J. A., Martínez Pillet, V., del Toro Iniesta, J. C., Domingo, V., Palacios, J., Knölker, M., Bello González, N., Berkefeld, T., Franz, M., Schmidt, W., and Title, A. M.: 2010, ApJ 723, L127

Solanki, S. K. and Fligge, M.: 1999, Geophys. Res. Lett. 26, 2465

Solanki, S. K. and Krivova, N. A.: 2004, Sol. Phys. 224, 197

Solanki, S. K. and Krivova, N. A.: 2011, Science 334, 916

Solanki, S. K., Krivova, N. A., and Haigh, J. D.: 2013, Annu. Rev. Astron. Astrophys. 51, 311

Solanki, S. K., Krivova, N. A., Schüssler, M., and Fligge, M.: 2002a, AE\&A 396, 1029 
Solanki, S. K., Riethmüller, T. L., Barthol, P., Danilovic, S., Deutsch, W., Doerr, H.-P., Feller, A., Gandorfer, A., Germerott, D., Gizon, L., Grauf, B., Heerlein, K., Hirzberger, J., Kolleck, M., Lagg, A., Meller, R., Tomasch, G., van Noort, M., Blanco Rodríguez, J., Gasent Blesa, J. L., Balaguer Jiménez, M., Del Toro Iniesta, J. C., López Jiménez, A. C., Orozco Suarez, D., Berkefeld, T., Halbgewachs, C., Schmidt, W., Álvarez-Herrero, A., Sabau-Graziati, L., Pérez Grande, I., Martínez Pillet, V., Card, G., Centeno, R., Knölker, M., and Lecinski, A.: 2017, ApJS 229, 2

Solanki, S. K., Schüssler, M., and Fligge, M.: 2000, Nature 408, 445

Solanki, S. K., Schüssler, M., and Fligge, M.: 2002b, AEFA 383(2), 706

Solanki, S. K., Usoskin, I. G., Kromer, B., Schüssler, M., and Beer, J.: 2004, Nature 431, 1084

Solomon, S., Qin, D., Manning, M., Chen, Z., Marquis, M., Averyt, K., Tignor, M., Miller, H., et al.: 2007, Contribution of working group I to the fourth assessment report of the intergovernmental panel on climate change, 2007

Sonett, C. P. and Finney, S. A.: 1990, Philosophical Transactions of the Royal Society of London Series A 330, 413

Spiegel, E. A. and Zahn, J.-P.: 1992, A\&A 265, 106

Spruit, H.: 2000, Space Sci. Rev. 94, 113

Spruit, H. C.: 1976, Sol. Phys. 50, 269

Spruit, H. C. and Roberts, B.: 1983, Nature 304, 401

Stanev, T.: 2010, High Energy Cosmic Rays, Springer Praxis Books, Springer Berlin Heidelberg

Steig, E. J., Polissar, P. J., Stuiver, M., Grootes, P. M., and Finkel, R. C.: 1996, Geophys. Res. Lett. 23, 523

Steinhilber, F., Abreu, J. A., and Beer, J.: 2008, Astrophysics and Space Sciences Transactions $\mathbf{4}, 1$

Steinhilber, F., Abreu, J. A., Beer, J., Brunner, I., Christl, M., Fischer, H., Heikkila, U., Kubik, P. W., Mann, M., McCracken, K. G., Miller, H., Miyahara, H., Oerter, H., and Wilhelms, F.: 2012, Proc. Natl. Acad. Sci. 109, 5967

Steinhilber, F., Beer, J., and Fröhlich, C.: 2009, Geophys. Res. Lett. 36, L19704

Stephenson, F. R.: 1990, Philosophical Transactions of the Royal Society of London Series A 330, 499

Stewart, J. Q. and Panofsky, H. A. A.: 1938, ApJ 88, 385

Stix, M.: 2002, The sun: an introduction, Berlin : Springer 
Stuiver, M.: 1961, J. Geophys. Res. 66, 273

Stuiver, M. and Braziunas, T. F.: 1989, Nature 338, 405

Stuiver, M. and Braziunas, T. F.: 1993, The Holocene 3, 289

Stuiver, M., Braziunas, T. F., Becker, B., and Kromer, B.: 1991, Quaternary Research 35, 1

Stuiver, M., Grootes, P. M., and Braziunas, T. F.: 1995, Quaternary Research 44, 341

Stuiver, M. and Polach, H. A.: 1977, Radiocarbon 19(3), 355

Stuiver, M. and Quay, P. D.: 1980, Science 207, 11

Suess, H. E.: 1955, Science 122, 415

Suess, H. E.: 1980, Radiocarbon 22(2), 200

Sukhodolov, T., Usoskin, I., Rozanov, E., Asvestari, E., Ball, W. T., Curran, M. A. J., Fischer, H., Kovaltsov, G., Miyake, F., Peter, T., Plummer, C., Schmutz, W., Severi, M., and Traversi, R.: 2017, Sci. Rep. 7, 45257

Svalgaard, L.: 2011, Proceedings of the International Astronomical Union 7(S286), 27-33

Svalgaard, L., Cagnotti, M., and Cortesi, S.: 2017, Sol. Phys. 292, 34

Svalgaard, L. and Schatten, K. H.: 2016, Sol. Phys. 291, 2653

Svensmark, H. and Friis-Christensen, E.: 1997, Jour. of Atmos. and Sol.-Terres. Phys. 59, 1225

Svensmark, J., Enghoff, M. B., Shaviv, N. J., and Svensmark, H.: 2016, J. Geophys. Res. $\mathbf{1 2 1}, 8152$

Tapping, K. F.: 1987, J. Geophys. Res. 92, 829

Tapping, K. F.: 2013, Space Weather 11, 394

Taylor, R. E. (ed.): 1987, Radiocarbon Dating an Archaeological Perspective, Academic Press

Thompson, M. J., Toomre, J., Anderson, E. R., Antia, H. M., Berthomieu, G., Burtonclay, D., Chitre, S. M., Christensen-Dalsgaard, J., Corbard, T., De Rosa, M., Genovese, C. R., Gough, D. O., Haber, D. A., Harvey, J. W., Hill, F., Howe, R., Korzennik, S. G., Kosovichev, A. G., Leibacher, J. W., Pijpers, F. P., Provost, J., Rhodes, Jr., E. J., Schou, J., Sekii, T., Stark, P. B., and Wilson, P. R.: 1996, Science 272, 1300

Title, A.: 2000, in Astronomy, physics and chemistry of $\mathrm{H}_{3}^{+}$, Vol. 358 of Philosophical Transactions of the Royal Society of London Series A, pp 657-668 
Toptygin, I. N.: 1985, Cosmic rays in interplanetary magnetic fields, Kluwer Academic Publishers, Dordrecht

Torres, D. F., Boldt, E., Hamilton, T., and Loewenstein, M.: 2002, Phys. Rev. D 66, 023001

Unruh, Y. C., Krivova, N. A., Solanki, S. K., Harder, J. W., and Kopp, G.: 2008, AEAA 486, 311

Unruh, Y. C., Solanki, S. K., and Fligge, M.: 1999, AEA 345, 635

Usoskin, I. G.: 2013, Living Rev. Sol. Phys. 10, 1

Usoskin, I. G.: 2017, Living Rev. Sol. Phys. 14, 3

Usoskin, I. G., Alanko-Huotari, K., Kovaltsov, G. A., and Mursula, K.: 2005, J. Geophys. Res. 110(A9), A12108

Usoskin, I. G., Arlt, R., Asvestari, E., Hawkins, E., Käpylä, M., Kovaltsov, G. A., Krivova, N., Lockwood, M., Mursula, K., O’Reilly, J., Owens, M., Scott, C. J., Sokoloff,

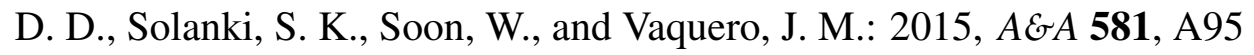

Usoskin, I. G., Field, C. V., Schmidt, G. A., LeppäNen, A.-P., Aldahan, A., Kovaltsov, G. A., Possnert, G., and Ungar, R. K.: 2009, J. Geophys. Res. 114, D06108

Usoskin, I. G., Gallet, Y., Lopes, F., Kovaltsov, G. A., and Hulot, G.: 2016a, AEA 5 587, A150

Usoskin, I. G., Gil, A., Kovaltsov, G. A., Mishev, A. L., and Mikhailov, V. V.: 2017, J. Geophys. Res. 122, 3875

Usoskin, I. G., Hulot, G., Gallet, Y., Roth, R., Licht, A., Joos, F., Kovaltsov, G. A., Thébault, E., and Khokhlov, A.: 2014, AESA 562, L10

Usoskin, I. G. and Kovaltsov, G. A.: 2008, J. Geophys. Res. 113(D12), D12107

Usoskin, I. G. and Kovaltsov, G. A.: 2012, ApJ 757, 92

Usoskin, I. G., Kovaltsov, G. A., and Chatzistergos, T.: 2016b, Sol. Phys. 291, 3793

Usoskin, I. G., Kovaltsov, G. A., Lockwood, M., Mursula, K., Owens, M. J., and Solanki, S. K.: 2016c, Sol. Phys. 291, 2685

Usoskin, I. G. and Kromer, B.: 2005, Radiocarbon 47(1), 31

Usoskin, I. G., Kromer, B., Ludlow, F., Beer, J., Friedrich, M., Kovaltsov, G. A., Solanki, S. K., and Wacker, L.: 2013, A\&A 552, L3

Usoskin, I. G., Mursula, K., and Kovaltsov, G. A.: 2000, AE\&A 354, L33

Usoskin, I. G., Mursula, K., and Kovaltsov, G. A.: 2001, J. Geophys. Res. 106, 16039 
Usoskin, I. G., Mursula, K., Solanki, S. K., Schüssler, M., and Kovaltsov, G. A.: 2002, J. Geophys. Res. 107(11)

Usoskin, I. G., Solanki, S. K., and Korte, M.: 2006a, Geophys. Res. Lett. 33, L08103

Usoskin, I. G., Solanki, S. K., and Kovaltsov, G. A.: 2007, AEFA 471, 301

Usoskin, I. G., Solanki, S. K., Kovaltsov, G. A., Beer, J., and Kromer, B.: 2006b, Geophys. Res. Lett. 33(8), L08107

Usoskin, I. G., Solanki, S. K., Schüssler, M., Mursula, K., and Alanko, K.: 2003, Phys. Rev. Lett. 91(21), 211101

van Ballegooijen, A. A., Asgari-Targhi, M., Cranmer, S. R., and DeLuca, E. E.: 2011, ApJ 736, 3

van Ballegooijen, A. A., Cartledge, N. P., and Priest, E. R.: 1998, ApJ 501, 866

van Loon, H., Meehl, G. A., and Shea, D. J.: 2007, J. Geophys. Res. 112, D02108

Vanlommel, P., Cugnon, P., van der Linden, R. A. M., Berghmans, D., and Clette, F.: 2004, Sol. Phys. 224, 113

Vaquero, J. M.: 2007, Advances in Space Research 40, 929

Vaquero, J. M., Kovaltsov, G. A., Usoskin, I. G., Carrasco, V. M. S., and Gallego, M. C.: 2015, AEA 577, A71

Vaquero, J. M. and Trigo, R. M.: 2014, Sol. Phys. 289, 803

Vaquero, J. M., Trigo, R. M., and Gallego, M. C.: 2012, Sol. Phys. 277, 389

Vasiliev, S. S. and Dergachev, V. A.: 2002, Annales Geophysicae 20, 115

Vázquez, M., Vaquero, J. M., Gallego, M. C., Roca Cortés, T., and Pallé, P. L.: 2016, Sol. Phys. 291, 613

Veres, D., Bazin, L., Landais, A., Toyé Mahamadou Kele, H., Lemieux-Dudon, B., Parrenin, F., Martinerie, P., Blayo, E., Blunier, T., Capron, E., Chappellaz, J., Rasmussen, S. O., Severi, M., Svensson, A., Vinther, B., and Wolff, E. W.: 2013, Clim. Past 9, 1733

Vernazza, J. E., Avrett, E. H., and Loeser, R.: 1981, ApJS 45, 635

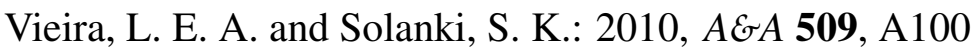

Vieira, L. E. A., Solanki, S. K., Krivova, N. A., and Usoskin, I. G.: 2011, AE्FA 531, A6

Vinther, B. M., Clausen, H. B., Johnsen, S. J., Rasmussen, S. O., Andersen, K. K., Buchardt, S. L., Dahl-Jensen, D., Seierstad, I. K., Siggaard-Andersen, M.-L., Steffensen, J. P., Svensson, A., Olsen, J., and Heinemeier, J.: 2006, J. Geophys. Res. 111, D13102

Vitinskij, Y. I., Kopetskij, M., and Kuklin, G. V.: 1986, Statistics of the spot-forming activity of the sun. 
Vögler, A., Shelyag, S., Schüssler, M., Cattaneo, F., Emonet, T., and Linde, T.: 2005, $A \mathcal{E} A$ 429, 335

Volobuev, D. M.: 2009, Sol. Phys. 258, 319

Vonmoos, M., Beer, J., and Muscheler, R.: 2006, J. Geophys. Res. 111, A10105

Waldmeier, M.: 1935, Astron. Mitt. Zurich 14(133), 105

Waldmeier, M.: 1939, Astron. Mitt. Zurich 14(138), 470

Waldmeier, M.: 1961, The sunspot-activity in the years 1610-1960, Schulthess and Company AG, Zürich

Wang, J., Zhou, G., Jin, C., and Li, H.: 2012, Sol. Phys. 278, 299

Wang, Y. M., Lean, J. L., and Sheeley, Jr., N. R.: 2005, ApJ 625(1), 522

Wang, Y.-M., Nash, A. G., and Sheeley, Jr., N. R.: 1989, Science 245, 712

Waters, L. S., McKinney, G. W., Durkee, J. W., Fensin, M. L., Hendricks, J. S., James, M. R., Johns, R. C., and Pelowitz, D. B.: 2007, in M. Albrow and R. Raja (eds.), Hadronic Shower Simulition Workshop, Vol. 896 of American Institute of Physics Conference Series, pp 81-90

Webber, W. R. and Higbie, P. R.: 2003, J. Geophys. Res. 108, 1355

Webber, W. R. and Higbie, P. R.: 2009, J. Geophys. Res. 114, A02103

Webber, W. R., Higbie, P. R., and McCracken, K. G.: 2007, J. Geophys. Res. 112, A10106

Weber, M., Burrows, J. P., and Cebula, R. P.: 1998, Sol. Phys. 177, 63

Wenzler, T., Solanki, S. K., and Krivova, N. A.: 2005, AEA A 432, 1057

Wenzler, T., Solanki, S. K., Krivova, N. A., and Fluri, D. M.: 2004, AEAA 427, 1031

Wenzler, T., Solanki, S. K., Krivova, N. A., and Fröhlich, C.: 2006, A\&A 460, 583

Wieland, E., Santschi, P. H., and Beer, J.: 1991, J. Geophys. Res. 96, 17

Willamo, T., Usoskin, I. G., and Kovaltsov, G. A.: 2017, AE्FA 601, A109

Willson, R. C.: 1997, Science 277, 1963

Willson, R. C., Gulkis, S., Janssen, M., Hudson, H. S., and Chapman, G. A.: 1981, Science 211, 700

Willson, R. C. and Hudson, H. S.: 1991, Nature 351, 42

Willson, R. C. and Mordvinov, A. V.: 2003, Geophys. Res. Lett. 30, 1199

Wilson, P. R., Altrocki, R. C., Harvey, K. L., Martin, S. F., and Snodgrass, H. B.: 1988, Nature 333, 748 
Wilson, R. M.: 1987, J. Geophys. Res. 92(A9), 10101

Wilson, R. M., Hathaway, D. H., and Reichmann, E. J.: 1998, J. Geophys. Res. 103, 6595

Wittmann, A. D. and Xu, Z. T.: 1987, AEFAS 70, 83

Wolf, R.: 1850, Astronomische Mitteilungen der Eidgenössischen Sternwarte Zurich 1, 3

Woods, T. N., Chamberlin, P. C., Harder, J. W., Hock, R. A., Snow, M., Eparvier, F. G., Fontenla, J., McClintock, W. E., and Richard, E. C.: 2009, Geophys. Res. Lett. 36, L01101

Woods, T. N., Eparvier, F. G., Fontenla, J., Harder, J., Kopp, G., McClintock, W. E., Rottman, G., Smiley, B., and Snow, M.: 2004, Geophys. Res. Lett. 31, L10802

Woods, T. N., Snow, M., Harder, J., Chapman, G., and Cookson, A.: 2015, Sol. Phys. 290, 2649

Woods, T. N., Tobiska, W. K., Rottman, G. J., and Worden, J. R.: 2000, J. Geophys. Res. $\mathbf{1 0 5}, 27195$

Wu, C.-J., Krivova, N. A., Solanki, S. K., and Usoskin, I. G.: 2018a, AEFA(submitted)

Wu, C. J., Usoskin, I. G., Krivova, N., Kovaltsov, G. A., Baroni, M., Bard, E., and Solanki, S. K.: 2018b, ArXive-prints

Xapsos, M. A. and Burke, E. A.: 2009, Sol. Phys. 257, 363

Yang, S., Odah, H., and Shaw, J.: 2000, Geophys. J. Int. 140, 158

Yang, S. and Zhang, J.: 2014, ApJ 781, 7

Yau, K. K. C.: 1988, Ph.D. thesis, Durham University

Yau, K. K. C. and Stephenson, F. R.: 1988, QJRAS 29, 175

Yeo, K. L., Ball, W. T., Krivova, N. A., Solanki, S. K., Unruh, Y. C., and Morrill, J.: 2015, J. Geophys. Res. 120(8), 6055

Yeo, K. L., Krivova, N. A., and Solanki, S. K.: 2014a, Space Sci. Rev. 186, 137

Yeo, K. L., Krivova, N. A., and Solanki, S. K.: 2017a, J. Geophys. Res. 122(4), 3888

Yeo, K. L., Krivova, N. A., Solanki, S. K., and Glassmeier, K. H.: 2014b, AE्FA 570, A85

Yeo, K. L., Solanki, S. K., and Krivova, N. A.: 2013, AEAA 550, A95

Yeo, K. L., Solanki, S. K., Norris, C. M., Beeck, B., Unruh, Y. C., and Krivova, N. A.: 2017b, Phys. Rev. Lett. 119(9)

Yiou, F., Raisbeck, G. M., Baumgartner, S., Beer, J., Hammer, C., Johnsen, S., Jouzel, J., Kubik, P. W., Lestringuez, J., Stiévenard, M., Suter, M., and Yiou, P.: 1997, J. Geophys. Res. 102, 26783 
Zachilas, L. and Gkana, A.: 2015, Sol. Phys. 290, 1457

Zhao, J. and Li, H.: 2012, Res. in Astron. and Astrophys. 12, 1681

Zharkova, V. V., Shepherd, S. J., Popova, E., and Zharkov, S. I.: 2015, Sci. Rep. 5, 15689

Zięba, S. and Nieckarz, Z.: 2014, Sol. Phys. 289, 2705

Zolotova, N. V. and Ponyavin, D. I.: 2014, J. Geophys. Res. 119, 3281

Zolotova, N. V. and Ponyavin, D. I.: 2015, ApJ 800, 42

Zwaan, C.: 1985, Sol. Phys. 100, 397 



\section{A Appendix}

\section{A.1 Derivation of the magnetic flux in the SATIRE-M model}

This appendix is to derive the magnetic fluxes in the SATIRE-M model, which is based partially on the SATIRE-T model, by taking the decadal resolution of the input data into account. Therefore, the ODEs in the SATIRE-T model are first averaged decadally. The Eqs. (4.2) - (4.4) are then written as

$$
\begin{gathered}
\left\langle\frac{d \varphi_{\mathrm{act}}}{d t}\right\rangle=\left\langle\varepsilon_{\mathrm{act}}\right\rangle-\left\langle\frac{\varphi_{\mathrm{act}}}{\tau_{\mathrm{act}}^{0}}\right\rangle-\left\langle\frac{\varphi_{\mathrm{act}}}{\tau_{\mathrm{act}}^{\mathrm{s}}}\right\rangle-\left\langle\frac{\varphi_{\mathrm{act}}}{\tau_{\mathrm{act}}^{\mathrm{r}}}\right\rangle, \\
\left\langle\frac{d \varphi_{\mathrm{eph}}}{d t}\right\rangle=\left\langle\varepsilon_{\mathrm{eph}}\right\rangle-\left\langle\frac{\varphi_{\mathrm{eph}}}{\tau_{\mathrm{eph}}^{0}}\right\rangle-\left\langle\frac{\varphi_{\mathrm{eph}}}{\tau_{\mathrm{eph}}^{\mathrm{s}}}\right\rangle, \\
\left\langle\frac{d \varphi_{\mathrm{open}}^{\mathrm{r}}}{d t}\right\rangle=\left\langle\frac{\varphi_{\mathrm{act}}}{\tau_{\mathrm{act}}^{\mathrm{r}}}\right\rangle-\left\langle\frac{\varphi_{\mathrm{open}}^{\mathrm{r}}}{\tau_{\mathrm{open}}^{\mathrm{r}}}\right\rangle,
\end{gathered}
$$

where the use of the subscriptions are identical as in the thesis context (Chap. 4). On the decadal time scale, the fluxes of ARs, ERs and the rapid open fluxes are assumed to evolve steady, namely:

$$
\left\langle\frac{d \varphi_{\mathrm{act}}}{d t}\right\rangle=0, \quad\left\langle\frac{d \varphi_{\mathrm{eph}}}{d t}\right\rangle=0, \quad\left\langle\frac{d \varphi_{\mathrm{open}}^{\mathrm{r}}}{d t}\right\rangle=0
$$

The Eqs. (A.1) - (A.3) can therefore be written as

$$
\begin{aligned}
\left\langle\varepsilon_{\mathrm{act}}\right\rangle & =\left\langle\frac{\varphi_{\mathrm{act}}}{\tau_{\mathrm{act}}}\right\rangle, \\
\left\langle\varepsilon_{\mathrm{eph}}\right\rangle & =\left\langle\frac{\varphi_{\mathrm{eph}}}{\tau_{\mathrm{eph}}}\right\rangle, \\
\left\langle\frac{\varphi_{\mathrm{act}}}{\tau_{\mathrm{act}}^{\mathrm{r}}}\right\rangle & =\left\langle\frac{\varphi_{\mathrm{open}}^{\mathrm{r}}}{\tau_{\mathrm{open}}^{\mathrm{r}}}\right\rangle,
\end{aligned}
$$

where

$$
\frac{1}{\tau_{\mathrm{act}}}=\frac{1}{\tau_{\mathrm{act}}^{0}}+\frac{1}{\tau_{\mathrm{act}}^{s}}+\frac{1}{\tau_{\mathrm{act}}^{r}},
$$

and

$$
\frac{1}{\tau_{\mathrm{eph}}}=\frac{1}{\tau_{\mathrm{eph}}^{0}}+\frac{1}{\tau_{\mathrm{act}}^{s}} .
$$


Next, since the open magnetic flux is a combination of both the rapidly decaying open flux, $\varphi_{\text {open }}^{r}$, and the slowly decaying open flux, $\varphi_{\text {open }}^{s}$, the total open magnetic flux is described as

$$
\varphi_{\mathrm{open}}=\varphi_{\mathrm{open}}^{r}+\varphi_{\mathrm{open}}^{s}
$$

or

$$
\frac{\varphi_{\mathrm{open}}}{d t}=\frac{\varphi_{\mathrm{open}}^{r}}{d t}+\frac{\varphi_{\mathrm{open}}^{s}}{d t} .
$$

By substituting the Eq. (4.5), the Eq. (A.11) can be re-written as

$$
\frac{\varphi_{\mathrm{open}}}{d t}=\frac{\varphi_{\mathrm{open}}^{r}}{d t}+\frac{\varphi_{\mathrm{act}}}{\tau_{\mathrm{act}}^{\mathrm{s}}}+\frac{\varphi_{\mathrm{eph}}}{\tau_{\mathrm{eph}}^{\mathrm{s}}}-\frac{\varphi_{\mathrm{open}}^{\mathrm{s}}}{\tau_{\mathrm{open}}^{\mathrm{s}}},
$$

or

$$
\frac{\varphi_{\mathrm{open}}}{d t}=\frac{\varphi_{\mathrm{open}}^{r}}{d t}+\frac{\varphi_{\mathrm{act}}}{\tau_{\mathrm{act}}^{\mathrm{s}}}+\frac{\varphi_{\mathrm{eph}}}{\tau_{\mathrm{eph}}^{\mathrm{s}}}+\frac{\varphi_{\mathrm{open}}^{r}}{\tau_{\mathrm{open}}^{\mathrm{s}}}-\frac{\varphi_{\mathrm{open}}}{\tau_{\mathrm{open}}^{\mathrm{s}}} .
$$

The decadal average of the Eq. (A.13) is

$$
\left\langle\frac{\varphi_{\mathrm{open}}}{d t}\right\rangle=\left\langle\frac{\varphi_{\mathrm{open}}^{r}}{d t}\right\rangle+\left\langle\frac{\varphi_{\mathrm{act}}}{\tau_{\mathrm{act}}^{\mathrm{s}}}\right\rangle+\left\langle\frac{\varphi_{\mathrm{eph}}}{\tau_{\mathrm{eph}}^{\mathrm{s}}}\right\rangle+\left\langle\frac{\varphi_{\mathrm{open}}^{r}}{\tau_{\mathrm{open}}^{\mathrm{s}}}\right\rangle-\left\langle\frac{\varphi_{\mathrm{open}}}{\tau_{\mathrm{open}}^{\mathrm{s}}}\right\rangle .
$$

Secondly, by averaging the Eq. (4.6) decadally, the emergence rate of the AR can be described as

$$
\varepsilon_{\mathrm{act}}=\frac{\varepsilon^{\max , 21}}{R^{\max , 21}}\langle R\rangle,
$$

which is then substituted into the Eq. (A.5). Eq. (A.5) is hence re-written as

$$
\left\langle\varphi_{\text {act }}\right\rangle=\tau_{\text {act }} \frac{\varepsilon^{\max , 21}}{R^{\max , 21}}\langle R\rangle .
$$

By taking the definition of the AR emergence rate (Eq. (4.6)) into the the Eq. (4.7), the ER emergence rate is then re-written as

$$
\varepsilon_{\mathrm{eph}}(t)=X \sum_{n=1} \frac{\varepsilon^{\max , 21}}{R^{\max , 21}} R^{\max , 21} g^{n},
$$

where $R^{\max , 21}=k\left\langle R_{\mathrm{g}}\right\rangle$. The Eq. (A.17) can further be written as

$$
\varepsilon_{\mathrm{eph}}(t)=\frac{\varepsilon^{\max , 21}}{R^{\max , 21}} X k \sum_{n=1}\langle R\rangle g^{n} .
$$

At the maximum timing of each ER cycle, the ER function $g^{n}$ can be seen as one. Hence, by combining the Eq. (A.17) and the Eq. (A.6), the Eq. (A.17) can be written as follow

$$
\left\langle\varphi_{\mathrm{eph}}\right\rangle=\tau_{\mathrm{eph}} \frac{\varepsilon^{\max , 21}}{R^{\max , 21}} X k\langle R\rangle .
$$

Finally, the evolution of rapidly decaying open magnetic flux is assumed to be in a steady state, i.e., $\left\langle\frac{d \varphi_{\text {open }}^{r}}{d t}\right\rangle=0$. The Eq. (A.14) is then re-written as

$$
\left\langle\frac{\varphi_{\mathrm{open}}}{d t}\right\rangle=\frac{\left\langle\varphi_{\mathrm{open}}\right\rangle_{j+1}}{d t}-\frac{\left\langle\varphi_{\mathrm{open}}\right\rangle_{j}}{d t}=\left\langle\frac{\varphi_{\mathrm{act}}}{\tau_{\mathrm{act}}^{\mathrm{s}}}\right\rangle+\left\langle\frac{\varphi_{\mathrm{eph}}}{\tau_{\mathrm{eph}}^{\mathrm{s}}}\right\rangle+\left\langle\frac{\varphi_{\mathrm{open}}^{\mathrm{r}}}{\tau_{\mathrm{open}}^{\mathrm{s}}}\right\rangle-\left\langle\frac{\varphi_{\mathrm{open}}}{\tau_{\mathrm{open}}^{\mathrm{s}}}\right\rangle,
$$


where $j$ represents the $j$-th decade. We then combine the Eq. (A.7) and the Eq. (A.20), the original Eq. (A.20) is then written as

$$
\frac{\left\langle\varphi_{\mathrm{open}}\right\rangle_{j+1}}{d t}+\frac{\left\langle\varphi_{\mathrm{open}}\right\rangle_{j}}{\tau_{\mathrm{open}}^{\mathrm{s}}}-\frac{\left\langle\varphi_{\mathrm{open}}\right\rangle_{j}}{d t}=\left\langle\frac{\varphi_{\mathrm{act}}}{\tau_{\mathrm{act}}^{\mathrm{s}}}\right\rangle_{j}+\left\langle\frac{\varphi_{\mathrm{eph}}}{\tau_{\mathrm{eph}}^{\mathrm{s}}}\right\rangle_{j}+\frac{\tau_{\mathrm{open}}^{\mathrm{r}}}{\tau_{\mathrm{open}}^{\mathrm{r}} \tau_{\mathrm{open}}^{\mathrm{s}}}\left\langle\varphi_{\mathrm{act}}\right\rangle_{j} .
$$

or

$$
\frac{\left\langle\varphi_{\mathrm{open}}\right\rangle_{j+1}}{d t}+\frac{\left\langle\varphi_{\mathrm{open}}\right\rangle_{j}}{\tau_{1}}=\left(\frac{1}{\tau_{\mathrm{act}}^{\mathrm{s}}}+\frac{\tau_{\mathrm{open}}^{\mathrm{r}}}{\tau_{\mathrm{act}}^{\mathrm{r}} \tau_{\mathrm{open}}^{\mathrm{s}}}\right)\left\langle\varphi_{\mathrm{act}}\right\rangle_{j}+\frac{1}{\tau_{\mathrm{eph}}^{\mathrm{s}}}\left\langle\varphi_{\mathrm{eph}}\right\rangle_{j},
$$

where

$$
\frac{1}{\tau_{1}}=\frac{1}{\tau_{\mathrm{open}}^{s}}-\frac{1}{d t}
$$

By applying Eq. (A.16) and Eq. (A.19), the Eq. (A.22) is finally written as

$$
\frac{\left\langle\varphi_{\mathrm{open}}\right\rangle_{j+1}}{d t}+\frac{\left\langle\varphi_{\mathrm{open}}\right\rangle_{j}}{\tau_{1}}=\left[\left(\frac{1}{\tau_{\mathrm{act}}^{\mathrm{s}}}+\frac{\tau_{\mathrm{open}}^{\mathrm{r}}}{\tau_{\mathrm{open}}^{\mathrm{s}} \tau_{\mathrm{act}}^{\mathrm{r}}}\right) \tau_{\mathrm{act}}+\frac{k X \tau_{\mathrm{eph}}}{\tau_{\mathrm{eph}}^{\mathrm{s}}}\right] \frac{\varepsilon_{\mathrm{act}}^{\mathrm{max}, 21}}{R^{\mathrm{max}, 21}}\langle R\rangle_{j},
$$

or

$$
\frac{\left\langle\varphi_{\mathrm{open}}\right\rangle_{j+1}}{d t}+\frac{\left\langle\varphi_{\mathrm{open}}\right\rangle_{j}}{\tau_{1}}=c\langle R\rangle_{j}
$$

where

$$
c=\left[\left(\frac{1}{\tau_{\mathrm{act}}^{\mathrm{s}}}+\frac{\tau_{\mathrm{open}}^{\mathrm{r}}}{\tau_{\mathrm{open}}^{\mathrm{s}} \tau_{\mathrm{act}}^{\mathrm{r}}}\right) \tau_{\mathrm{act}}+\frac{k X \tau_{\mathrm{eph}}}{\tau_{\mathrm{eph}}^{\mathrm{s}}}\right] \frac{\varepsilon_{\mathrm{act}}^{\mathrm{max}, 21}}{R^{\mathrm{max}, 21}},
$$

The numerical value $k$ was found by Usoskin et al. (2007) to be $2.2 \pm 0.4$. In this work, we take $k=2.2$, Finally, by rearranging the Eq. (A.25), we get

$$
\langle R\rangle_{j}=a\left\langle\varphi_{\mathrm{open}}\right\rangle_{j}+b\left\langle\varphi_{\mathrm{open}}\right\rangle_{j+1},
$$

where

$$
a=\frac{1}{\tau_{1}}
$$

and

$$
b=\frac{1}{c \Delta t} \text {. }
$$

The $\Delta t$ is the sample resolution, which in this thesis is 10 years based on the cosmogenic isotope data sampling. 


\section{A.2 Derivation of the solar irradiance in the SATIRE-M model}

As introduced in the main content of the thesis, calculating the solar spectral irradiance requires knowledge of the surface coverages (filling factors) of the photospheric components. In this appendix, we will derive how to obtain the filling factors and to calculate the solar irradiance on decadal time scale.

On decadal time scale, the decadally-averaged sunspot filling factor and the decadallyaveraged sunspot number can be simplified as a linear relationship, as

$$
\left\langle\alpha_{\mathrm{s}}\right\rangle_{j}=A_{1}\langle R\rangle_{j}+A_{2},
$$

where the coefficients $A_{1}$ and $A_{2}$ are the proportionality and offset, respectively.

By taking the ratio of umbral-penumbral area 1:4 into account (Solanki 2003) and, substituting in the Eq. (A.27), the Eq. (A.30) can be separated as two parts (one for umbra and one for penumbra). They are written as

$$
\left\langle\alpha_{\mathrm{u}}\right\rangle_{j}=0.2\left\langle\alpha_{\mathrm{s}}\right\rangle_{j}=a_{\mathrm{u}}\left\langle\varphi_{\mathrm{open}}\right\rangle_{j}+b_{\mathrm{u}}\left\langle\varphi_{\mathrm{open}}\right\rangle_{j+1}+c_{\mathrm{u}},
$$

and

$$
\left\langle\alpha_{\mathrm{p}}\right\rangle_{j}=0.8\left\langle\alpha_{\mathrm{s}}\right\rangle_{j}=a_{\mathrm{p}}\left\langle\varphi_{\mathrm{open}}\right\rangle_{j}+b_{\mathrm{p}}\left\langle\varphi_{\mathrm{open}}\right\rangle_{j+1}+c_{\mathrm{p}}
$$

where

$$
\begin{array}{lll}
a_{\mathrm{u}}=0.2 A_{1} a, & b_{\mathrm{u}}=0.2 A_{1} b, & c_{\mathrm{u}}=0.2 A_{2}, \\
a_{\mathrm{p}}=0.8 A_{1} a, & b_{\mathrm{p}}=0.8 A_{1} b, & c_{\mathrm{p}}=0.8 A_{2} .
\end{array}
$$

For both faculae and network, we follow the same assumption in the SATIRE-T model (Chap. 4) that the filling factor is linearly proportional to the magnetic flux until the saturation flux, $B_{\text {sat }}$, is reached. Therefore, the decadally-averaged filling factor of network can be written as

$$
\left\langle\alpha_{\mathrm{n}}\right\rangle_{j}=\frac{\left\langle\varphi_{\mathrm{n}}\right\rangle_{j}}{S_{\odot} B_{\mathrm{sat}, \mathrm{n}}},
$$

where $S_{\odot}$ is the solar surface area and $B_{\text {sat,n }}$ is the saturation limit of network. Furthermore, since the network includes ERs and the OMF (Krivova et al. 2010), i.e, $\left\langle\varphi_{n}\right\rangle=$ $\left\langle\varphi_{\text {eph }}\right\rangle+\left\langle\varphi_{\text {open }}\right\rangle$, by taking the Eq. (A.19), the Eq. (A.35) is re-written as

$$
\left\langle\alpha_{\mathrm{n}}\right\rangle_{j}=a_{\mathrm{n}}\left\langle\varphi_{\mathrm{open}}\right\rangle_{j}+b_{\mathrm{n}}\left\langle\varphi_{\mathrm{open}}\right\rangle_{j+1},
$$

where

$$
\begin{gathered}
a_{\mathrm{n}}=1+W_{1} a, \\
b_{\mathrm{n}}=W_{1} b .
\end{gathered}
$$

where

$$
W_{1}=\frac{\tau_{\mathrm{eph}}}{S_{\odot} B_{\mathrm{sa}, \mathrm{n}}} \frac{\varepsilon^{\mathrm{max}, 21}}{R^{\text {max }, 21}} X k .
$$

Next, as same idea as used for network, the decadally-averaged filling factor of faculae is written as

$$
\left\langle\alpha_{\mathrm{f}}\right\rangle_{j}=\frac{\left\langle\varphi_{\mathrm{f}}\right\rangle_{j}}{S_{\odot} B_{\mathrm{sat}, \mathrm{f}}},
$$


where $B_{\mathrm{sat}, \mathrm{f}}$ is the saturation limit of faculae. Additionally, since the active regions consist of both sunspot and faculae, the decadally-averaged facular magnetic flux can be described as

$$
\left\langle\varphi_{\mathrm{f}}\right\rangle=\left\langle\varphi_{\mathrm{act}}\right\rangle-\left\langle\varphi_{\mathrm{u}}\right\rangle-\left\langle\varphi_{\mathrm{p}}\right\rangle
$$

By substituting the Eq. (A.16) and the Eq. (A.41) in the Eq. (A.40), the form of the decadally-averaged facular filling factor is

$$
\left\langle\alpha_{\mathrm{f}}\right\rangle_{j}=a_{\mathrm{f}}\left\langle\varphi_{\mathrm{open}}\right\rangle_{j}+b_{\mathrm{f}}\left\langle\varphi_{\mathrm{open}}\right\rangle_{j+1}+c_{\mathrm{f}}
$$

where

$$
\begin{gathered}
a_{\mathrm{f}}=W_{2} a \\
b_{\mathrm{f}}=W_{2} b \\
c_{\mathrm{f}}=-\left(\frac{0.2}{S_{\odot} B_{\text {sat }, \mathrm{f}}}+\frac{0.8}{B_{\mathrm{sat}, \mathrm{f}}}\right) A_{2} .
\end{gathered}
$$

where

$$
W_{2}=\left(\frac{\tau_{\text {act }} \varepsilon^{\max , 21}}{S_{\odot} R^{\max , 21}}-0.2\left[B_{\mathrm{u}}\right] A_{1}-0.8\left[B_{\mathrm{p}}\right] A_{1}\right) / B_{\text {sat }, \mathrm{f}} .
$$

The $\left[B_{\mathrm{u}}\right]$ and $\left[B_{\mathrm{p}}\right]$ are the mean field strength of umbra and penumbra, which are assumed as $1800 \mathrm{G}$ and $550 \mathrm{G}$, respectively. The values of the coefficients $\left(a_{\mathrm{u}, \mathrm{p}, \mathrm{n}, \mathrm{f}}, b_{\mathrm{u}, \mathrm{p}, \mathrm{n}, \mathrm{f}}\right.$ and $\left.c_{\mathrm{u}, \mathrm{p}, \mathrm{f}}\right)$ used in the SATIRE-M model are summarized in Table 4.3.

Lastly, by applying the obtained filling factors of these surface magnetic components (Eqs. A.31, A.32, A.36, A.42) in the concept of reconstructing the solar irradiance (Eq. 1.2), the final SSI on millennial time scale at any given time is

$$
\langle F(\lambda, t)\rangle_{j}=a_{\mathrm{F}}(\lambda)\left\langle\phi_{\mathrm{open}}\right\rangle_{j}+b_{\mathrm{F}}(\lambda)\left\langle\phi_{\mathrm{open}}\right\rangle_{j+1}+F_{\mathrm{q}}(\lambda),
$$

where

$$
a_{\mathrm{F}}(\lambda)=a_{\mathrm{u}} \Delta F_{\mathrm{u}}(\lambda)+a_{\mathrm{p}} \Delta F_{\mathrm{p}}(\lambda)+a_{\mathrm{n}} \Delta F_{\mathrm{n}}(\lambda)+a_{\mathrm{f}} \Delta F_{\mathrm{f}}(\lambda)
$$

and

$$
b_{\mathrm{F}}(\lambda)=b_{\mathrm{u}} \Delta F_{\mathrm{u}}(\lambda)+b_{\mathrm{p}} \Delta F_{\mathrm{p}}(\lambda)+b_{\mathrm{n}} \Delta F_{\mathrm{n}}(\lambda)+b_{\mathrm{f}} \Delta F_{\mathrm{f}}(\lambda) .
$$

The $\Delta F_{\mathrm{u}, \mathrm{p}, \mathrm{n}, \mathrm{f}}$ are the intensity contrasts of the magnetic components to the quiet Sun. 



\section{Publications}

\section{Refereed publications}

- Wu, C.-J., Ip, W.-H. and Huang, L.-C., 2015, A Study of Variability in the Frequency Distributions of the Superflares of G-type Stars Observed by the Kepler Mission, ApJ, 798, 92

- Kopp, G., Krivova, N. A., Wu, C.-J. and Lean, J., 2016, The Impact of the Revised Sunspot Record on Solar Irradiance Reconstructions, Sol. Phys., 291, 2951

- Jungclaus, J. H., Bard, E., Baroni, M., Braconnot, P., Cao, J., Chini, L. P., Egorova, T., Evans, M., González-Rouco, J. F., Goosse, H., Hurtt, G. C., Joos, F., Kaplan, J. O., Khodri, M., Klein Goldewijk, K., Krivova, N. A., LeGrande, A. N., Lorenz, S. J., Luterbacher, J., Man, W., Meinshausen, M., Moberg, A., Nehrbass-Ahles, C., Otto-Bliesner, B. I., Phipps, S. J., Pongratz, J., Rozanov, E., Schmidt, G. A., Schmidt, H., Schmutz, W., Schurer, A., Shapiro, A. I., Sigl, M., Smerdon, J. E., Solanki, S. K., Timmreck, C., Toohey, M., Usoskin, I. G., Wagner, S., Wu, C.-J., Yeo, K.-L., Zanchettin, D., Zhang, Q., Zorita, E., 2016, The PMIP4 contribution to CMIP6 - Part 3: the Last Millennium, Scientific Objective and Experimental Design for the PMIP4 past1000 simulations, Geosci. Model Dev. Disc., 10, 4005

- Wu, C.-J., Usoskin, I. G., Krivova, N. A., Kovaltsov, G. A, Baroni, M., Bard, E., Solanki, S. K., 2018, Solar activity over nine millennia: A consistent multi-proxy reconstruction, A\&A, accepted

- Wu, C.-J., Krivova, N. A., Solanki, S. K., Usoskin, I. G., 2018, Solar Total and Spectral Irradiance Reconstruction Over the Last 9000 Years, A\&A, submitted 


\section{Conference contributions}

- (Talk) Wu, C.-J., Usoskin, I. G., Krivova, N. A., Kovaltsov, G. A., Baroni, M., Bard ,E., Solanki, S. K., "A Multi-isotope Composite Record of Solar Activity and Irradiance Reconstruction over the last 9000 Years", EGU, Vienna, Austria, 8- 13 April 2018

- (Talk) Wu, C.-J., Krivova, N. A., Solanki, S. K., Usoskin, I. G., Kovaltsov, G. A., "Solar Spectral Irradiance over 9 Millennia from a Multi-isotope Composite Series”, IAU340 symposium, Jaipur, India, 18 - 24 February 2018

- (Talk) Wu, C.-J., Usoskin, I. G., Krivova, N. A., Kovaltsov, G. A., Solanki, S. K., "Solar Spectral Irradiance Reconstruction over 9 Millennia from a Composite ${ }^{14} \mathrm{C}$ and ${ }^{10}$ Be Series", AGU Fall meeting, New Orleans, USA, 11 - 15 December 2017

- (Talk \& Poster) Wu, C.-J., Usoskin, I. G., Krivova, N. A., Kovaltsov, G. A., "Solar Irradiance in the Holocene: A Consistent Multi-proxy Reconstruction", 1st PMIP4 conference, Stockholm, Sweden, 25 - 29 September 2017

- (Talk) Wu, C.-J., Usoskin, I. G., Krivova, N. A., Kovaltsov, G. A., "Solar Irradiance Reconstruction over Holocene: A Consistent Multi-proxy Series", German Astronomical Society, Göttingen, Germany, 18 - 22 September 2017

- (Talk) Wu, C.-J., Usoskin, I. G., Krivova, N. A., Kovaltsov, G. A., "Solar Irradiance Reconstruction over Holocene: A Consistent Multi-proxy Series", Rocks and Stars, Göttingen, Germany, 13 - 16 September 2017

- (Talk) Wu, C.-J., Krivova, N. A., Usoskin, I. G., Solanki, S. K., "Reconstruction of Solar Irradiance from Cosmogenic Isotope Data", Deutsche Physikalische Gesellschaft spring meeting, Bremen, Germany, 13 - 17 March 2017

- (Poster) Wu, C.-J., Krivova, N. A., Solanki, S. K., Usoskin, I. G., "Solar Total and Spectral Irradiance Reconstruction over Last 9000 Years", AGU Fall meeting, San Francisco, USA, 12 - 16 December 2016

- (Invited Talk) Wu, C.-J., Krivova, N. A., Solanki, S. K., Usoskin, I. G., "Reconstructions of Solar Irradiance on the Millennial Timescale", 13th European space weather weak, Oostende, Belgium, 14 - 18 November 2016

- (Talk) Wu, C.-J., Krivova, N. A., Solanki, S. K., Usoskin, I. G., "Solar Spectral Irradiance Reconstruction on Millennial Timescale", 1st Swiss SCOSTEP workshop, Bern, Switzerland, 4 - 5 October 2016

- (Poster) Wu, C.-J., Krivova, N. A., Solanki, S. K., Usoskin, I. G., "Solar total and spectral irradiance reconstruction over last 9000 years", Space Climate 6 Symposium, Levi, Finland, 4 - 7 April 2016 


\section{Acknowledgements}

三人行, 必有我師焉。

擇其善者而從之, 其不善者而改之。

- 孔子〈述而〉

In the last three years and a half, many people have been there for me, whenever I needed them. I wouldn't finish my study without their help.

First off, I would like to thank my supervisors: Natalie Krivova, Sami K. Solanki, and Ansgar Reiners, for giving me the chance to be their student. I also want to thank the Max Planck Institute for Solar System Research and the International Max Planck Research School for Solar System Science at the University of Göttingen, for funding my doctorate study.

I want to thank Natasha, for being incredibly patient with me and bringing me step by step from the very beginning. And I want thank Sami, for his insightful advises and helpful discussions at every meetings. I am really grateful for having such great supervisors, who gave me the opportunities to learn and to improve. Although they are super busy, they were always there to help me if I had any questions. I want to thank Natasha for reading and correcting my crappy English all the time. Through her comments, every single time, I could always improve my English better, know where the logic gaps are, and how to write more precisely and concisely. Also, I want to apologize to both Sami and Natasha, for giving them so much pressure reading my thesis in the last minute.

I would also like to thank my collaborator, Ilya Usoskin. Even I have only visited Oulu once for a very short week, we managed to accomplish and publish an amazing work together. Knowing I am not from the geophysics field and not very good at statistics, he always replied emails fast, and was always there to help me.

Kok Leng Yeo, who is an expert of the SATIRE model, has been helping me countless times. I always bothered him with tons of stupid questions. Also, as a newbie in the institute in the beginning, he introduced me to the coffee group (they are great company), which I would have never done by myself because I was simply too shy.

Moreover, I'd like to thank Sonja Schuh for her support and help with all the IMPRS-related hassles. Many thanks also go to Ines Dominitzki, who was always patiently helping me go through all the tiring bureaucracy and solve all the random troubles.

I also want to express my great appreciation to Robin Thor, Martin Bo Nielson and Kok Leng Yeo for proof-reading parts of this thesis, and correcting my grammar mistakes. I also like to thank Robin Thor for helping translating the summary from English to German.

I would like to offer my special thanks to my partner, Robin Thor, for always being there for me, sharing my happiness and listening to all my complains. With his chill temper and special German sense of humour, he can always calm me down whenever I feel nervous, angry or upset. Last but not least, I want to thank my family in Taiwan for their great great support, I would never have such achievements without their encouragement. 



\section{Curriculum vitae}

\section{Personal Information}

$\begin{array}{ll}\text { Surname } & \text { Wu } \\ \text { Name } & \text { Chi-Ju } \\ \text { Date of Birth } & \text { 13.02.1990 } \\ \text { Place of Birth } & \text { Taipei, Taiwan } \\ \text { Nationality } & \text { Taiwanese }\end{array}$

\section{Education}

2015 - 2018 Ph.D.

Max-Planck Institut für Sonnensystemforschung, Göttingen, Germany and Universität Göttingen, Göttingen, Germany

$2013-2014$ M.Sc.

Institute of Space Science, National Central University, Taiwan

$2009-2012$ B.Sc.

Atmospheric Science, National Central University, Taiwan

2007 - 2008 Theodor-Fliedner-Gymnasium, Düsseldorf, Germany

2005 - 2008 Taipei Municipal Song-Shan Senior High School, Taiwan 